\title{
Development of the Selective Coagulation Process
}

\author{
Principal Investigators
}

R.-H. Yoon and G. H. Luttrell

Virginia Center for Coal and Minerals Processing Department of Mining and Minerals Engineering Virginia Polytechnic Institute and State University Blacksburg, Virginia 24061-0258

Contract Number:

DE-AC22-90PC90174

\section{Project Manager:}

Richard Read

United States Department of Energy Pittsburgh Energy Technology Center

P.O. Box 10940

Pittsburgh, Pennsylvania 15236-0940

July 1992 


\begin{abstract}
ARSTRACT
The selective hydrophobic coagulation (SHC) process is based on the recent
\end{abstract} finding that hydrophobic particles can be selectively coagulated without using traditional agglomerating agents or flocculants. The driving force for the coagulation is the attractive energy between hydrophobic surfaces, an interaction that has been overlooked in classical colloid chemistry. In most cases, selective separations can be achieved using simple $\mathrm{pH}$ control to disperse the mineral matter, followed by recovery of the coal coagula using techniques that take advantage of the size enlargement.

In the present work, studies have been carried out to further investigate the fundamental mechanisms of the $S H C$ process and the parameters that affect the process of separating coal from the ash-forming minerals and pyritic sulfur. Studies have included direct force measurements of the attractive interaction between model hydrophobic surfaces, in-situ measurements of the size distributions of coagula formed under a variety of operating conditions, and development of a population balance model to describe the coagulation process. An extended DLVO colloid stability model which includes a hydrophobic interaction energy term has also been developed to explain the findings obtained from the experimental studies.

In addition to the fundamental studies, bench-scale process development test work has been performed to establish the best possible method of separating the coagula from dispersed mineral matter. Two types of separators, i.e., a sedimentation tank and a rotating drum screen, were examined in this study. The sedimentation tank proved to be the more efficient unit, achieving ash reductions as high as $60 \%$ in a single pass while recovering more than $90 \%$ of the combustible material. This device, which minimizes turbulence and coagula breakage, was used in subsequent test work to optimize design and operating parameters. 


\section{EXECUTIVE SUMMARY}

Recent studies carried out at the Virginia Center for Coal and Minerals Processing (VCCMP) have resulted in the development of a novel agglomeration process for upgrading ultrafine coal. This process, known as selective hydrophobic coagulation (SHC), is based on the new finding that hydrophobic coal particles can be selectively coagulated in the presence of dispersed mineral matter without the addition of traditional agglomerating agents or flocculants. The driving force for this process is believed to be the attractive hydrophobic force, the presence of which was recognized only recently in the scientific community. In most cases, $\mathrm{pH}$ control is all that is required to i) induce the coagulation of the coal particles, and ii) effectively disperse the particles of mineral matter.

The selective hydrophobic coagulation process has been studied at VCCMP since 1986 ("Advanced Systems for Producing Superclean Coal, " DOE Contract No. DEAC22-86PC91221). The overall objective of the current project was to develop an economical method of producing low-ash, low-sulfur coals using the SHC process. The work was subdivided into two phases: Phase I, studies of the fundamental mechanisms of the $S H C$ process and the parameters that affect the process of separating coal from the ash-forming minerals and pyritic sulfur; and Phase II, bench-scale process development test work to establish the best possible method of separating the coagula from the dispersed mineral matter.

The fundamental mechanisms of the selective hydrophobic coagulation process have been evaluated within the context of the extended DLVO theory. This theory is a modified version of the classical DLVO theory, which was developed independently by Derjaguin and Landau (1941) and Verwey and Overbeek (1948). The extended DLVO 
theory used in the present work was developed recently by $\mathrm{Xu}$ and Yoon (1989) and includes an additional attractive energy to account for hydrophobic interactions. The study of the fundamental mechanisms of the SHC process was carried out in nine subtasks areas and is summarized as follows:

In Task 2.1-Surface Forces Measurements, the existence of the hydrophobic interaction energy was demonstrated at a fundamental level through the measurement of total interaction forces for two highly hydrophobic model systems, i.e., mica surfaces coated with dimethyldioctadecylammonium bromide in $\mathrm{KBr}$ solutions and air bubbles/ methylated silica surfaces in $\mathrm{KCl}$ solutions. These systems were used because the surface roughness and opacity of coal prevent it from being used directly in the equipment required to measure these forces. This work has permitted the determination of the relative magnitudes of the three forces present in hydrophobic systems, namely, the dispersion, electrostatic, and hydrophobic interaction forces.

In Task 2.2 - Determination of Hydrophobic Interaction Parameters, cleaned samples of Elkhorn No. 3 and Pittsburgh No. 8 seam coals were subjected to batch coagulation tests to determine coagulation efficiencies as a function of solution $\mathrm{pH}$. The data showed that the maximum selectivity for the $S H C$ process occurs at a $\mathrm{pH}$ between 8 and 9 for highly hydrophobic coals. The upper limit of coagulation is dictated by dispersion of coal, while the lower limit is determined by hetero- and homo-coagulation of clay. Processing of less hydrophobic coals required lower $\mathrm{pH}$ conditions, and for oxidized coals, the addition of sequestering agents helped to minimize the non-selective coagulation of minerals and coal. It was also shown that a fresh Elkhorn No. 3 coal could be coagulated at $\zeta$-potentials as high as $-43 \mathrm{mV}$ due to the strong attractive hydrophobic force. This limiting $\zeta$-potential $\left(\zeta_{c}\right)$ was found to decrease in magnitude as 
the coal becomes less hydrophobic. The extended $D L V O$ theory was successfully used to explain these observations.

In Task 2.3 - Modeling of Hydrophobic Coagulation, a population balance model that requires a knowledge of the rates of particle aggregation and breakage was developed to describe the hydrophobic coagulation process. The rate of aggregation was estimated based on the extended DLVO theory, while the rate of breakage was determined by calculating the kinetic energies of the particles involved using the theories of mixing hydrodynamics. This information was combined into the general conservation equation to provide the population balance model, which was evaluated for a batch system. The size distributions predicted by the model compared well with experimentally determined coagula size distributions obtained under the same operating conditions.

In Task 2.4-Measurement of Coagula Size, an in-situ particle size analyzer was used to determine the coagula size distribution as a function of various operating conditions. In general, the median coagula size $\left(D_{s 0}\right)$, as measured with a Lasentec particle size analyzer, increased by nearly two orders of magnitude between completely dispersed and coagulated conditions. The maximum $D_{s 0}$ 's obtained using micronized samples of the Elkhorn No. 3 and Pittsburgh No. 8 coals were found to be 310 and $300 \mu \mathrm{m}$, respectively. These coagula sizes are comparable to those obtained in the presence of oil. The measured $D_{50}$ 's were found to decrease with increasing $\mathrm{pH}$ because of an increased electrostatic repulsive energy. Increased mixing intensity also sharply decreased the measured $D_{s o}$ 's. The high breakage rates suggest that the coagula formed by the $S H C$ process are weaker and more easily broken than those obtained using traditional oil agglomeration processes.

The process development test work was carried out i) to develop a better under- 
standing of the factors that control the effectiveness of the $S H C$ process, and ii) to evaluate various techniques for separating coal agglomerates formed by hydrophobic coagulation from unwanted mineral matter. These aims were implemented as follows:

In Task 3.1 - Sample Acquisition and Task 3.2 - Sample Characterization, samples of Elkhorn No. 3 and Pittsburgh No. 8 seam coal were obtained from a United Fuels Company preparation plant in Eastern Kentucky and a Consolidation Coal preparation plant in eastern Ohio. After being crushed to $-1 / 4$ inch, these samples were split into 100-gram lots and stored in air-tight bags in a freezer. Representative samples were analyzed for ash, volatiles, fixed carbon, total sulfur, sulfur forms and heating value. The liberation behavior with grinding of these two samples was assessed by SEM-IPS image analysis and by centrifugal sink-float analysis. These two methods gave similar results and indicated that mineral matter rejections near $80 \%$ were theoretically achievable at recovery levels approaching $90 \%$ for the particle sizes studied here. However, neither technique was found to be capable of discriminating between various types of submicron particles. As a result, the degree of liberation of the highly-micronized coal samples could not be accurately determined in the present work.

In Task 3.3 - Mixing Studies, the effects of mixing conditions on coagulation kinetics were investigated. In these studies, an in-situ Lasentec particle size analyzer was used to measure the mean coagula size as a function of mixer geometry and agitation speed. Five different configurations of baffled mixing cells and turbine impellers were examined. In all cases studied, the mean coagula size was found to decrease as the mixing intensity was increased. This suggests that the coagula breakage rate increases faster than the coagula growth rate when mechanical agitation is applied. It was also shown that the test data obtained using the various mixer geometries could be normalized into a single characteristic curve by plotting the mean size of the coagula as a function of 
the calculated kinetic energy of the particles. This finding should prove useful for the design and scale-up of the $S H C$ process.

In Task 3.4-Development of Methods for Enhancing the Separation of Coagula, various approaches were examined to enhance the settling rates of the coal coagula formed by the $S H C$ process. Higher settling rates would provide an increased throughput capacity of the process and lower the capital and operating costs. Approaches tested included seeding with coarse particles, adding hydrophobized magnetite (which could then be separated from the coal by magnetic separation methods), and adding hydrocarbon oils (to strengthen the integrity of the coal coagula). Test data obtained from batch sedimentation studies showed that the coagula settling rate increased significantly by adding large particles of coal or hydrophobized magnetite as seed particles. Unfortunately, the amount of the seed particles needed to double the coagula settling rates was found to be relatively large (i.e., generally $>50 \%$ by volume). Surprisingly, the addition of hydrocarbon oils had little impact on the settling velocity of the coagula. In fact, a small decrease in the measured coagula size was noted after the addition of kerosene and diesel fuel (although considerable data scatter was observed). This finding suggests that the addition of hydrocarbon oils may actually promote the formation of smaller, more compact coagula.

\section{In Task 3.5 - Separator Design and Construction and Task 3.6-Bench-scale}

Continuous Testing, two approaches to separate coagulated coal and dispersed mineral matter were considered. Of principal concern in this phase of the work was the relatively weak binding strength of the coal coagula and the resultant need to minimize turbulence in the separation device. A sedimentation tank and a rotating drum screen were developed and tested on a micronized sample Elkhorn No. 3 seam coal containing $22 \%$ ash. The sedimentation tank was found to be the more efficient unit, based on product ash content, combustible recovery, and separation efficiency values obtained for a given 
throughput per unit area. The sedimentation tank achieved ash reductions of as much as $60 \%$ in a single cleaning stage while recovering greater than $90 \%$ of the combustible material. The rotating drum screen was able to produce similar ash reductions, but recovery values were substantially lower (60 to $70 \%)$. Similar test work on a high-sulfur Pittsburgh No.8 seam coal was less successful, with very little rejection of ash. It is suspected that the presence of $\mathrm{Fe}^{3+}$ ions produced as a result of pyrite oxidation may have caused the non-selective coagulation of mineral matter and coal. Methods of mitigating this harmful effect will be examined further in future research efforts.

The optimum feed flow rate for an 11-inch diameter sedimentation tank was found to be between 250 and $350 \mathrm{ml} / \mathrm{min}$. This corresponds to approximately $1.5-2.1 \mathrm{lbs} / \mathrm{hr} / \mathrm{ft}^{2}$ of settling basin area at 3\% solids. Larger feed rates resulted in a decrease in recovery, whereas smaller feed rates increased the ash content in the product. These results were found to correlate well with the residence time of 30-40 minutes required for the settling of the coal coagula into the product process stream. The solids content corresponding to maximum separation efficiency was found to be $2 \%$. A higher solids content resulted in an increase in the product ash content, whereas a lower solids content resulted in a decrease in recovery. Particle size was also found to be an important process parameter. Recovery and product ash improved with decreasing particle size, presumably because of improved liberation and increased dominance of the surface forces at finer grind sizes.

In Task 3.7 - Process Optimization, a parametric test program based on a BoxBehnken experimental design was employed to examine the effects of several process variables (i.e., volumetric feed rate, feed solids content, mudline level and feed injection point) on the performance of an 8-inch diameter sedimentation tank. In general, low product ash contents were obtained from tests run with low feed rates and/or low feed solids contents and from tests where the distance between the feed point and the mudline 
was large. These conditions also generally produced lower weight recoveries and lower tailings ash contents.

Test data obtained from the parametric study were used to develop quadratic relationships for predicting the product ash content, combustible recovery, and separation efficiency. These relationships were then used to generate contour plots for these performance parameters and to determine the optimum operating point for the 8-inch diameter sedimentation tank. This study found that separation efficiency was maximized when the feed rate was high $(65 \mathrm{ml} / \mathrm{min})$, the solids content low $(2 \%)$ and the distance between the feed point and the mudline at its maximum $(6.825 \mathrm{~cm})$. This optimum was subsequently confirmed by repeat tests.

Although still in the early stages of its development, the $S H C$ process shows promise as a method of recovering fine coal from preparation plant refuse streams, especially those from the low-sulfur coal region of central Appalachia. The ability to recover clean fine coal without the extensive use of reagents is significant, though work is still required to develop high-capacity devices for this separation. This is the principal objective of the future project entitled, "Development of the Selective Hydrophobic Coagulation Process" (DOE No. DE-AC22-91PC91164). 


\section{TABLE OF CONTENTS}

ABSTRACT $\ldots \ldots \ldots \ldots \ldots \ldots \ldots \ldots \ldots \ldots \ldots \ldots \ldots \ldots \ldots \ldots \ldots$ iii

EXECUTIVE SUMMARY $\ldots \ldots \ldots \ldots \ldots \ldots \ldots \ldots \ldots \ldots \ldots$

TABLE OF CONTENTS $\ldots \ldots \ldots \ldots \ldots \ldots \ldots \ldots \ldots \ldots \ldots \ldots \ldots$ xiii

LIST OF FIGURES $\ldots \ldots \ldots \ldots \ldots \ldots \ldots \ldots \ldots \ldots \ldots \ldots \ldots \ldots$

LIST OF TABLES $\ldots \ldots \ldots \ldots \ldots \ldots \ldots \ldots \ldots \ldots \ldots \ldots \ldots \ldots \ldots \ldots \ldots$

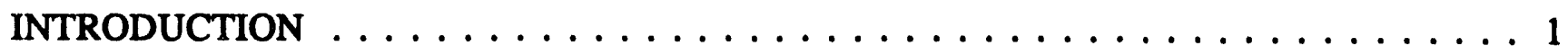

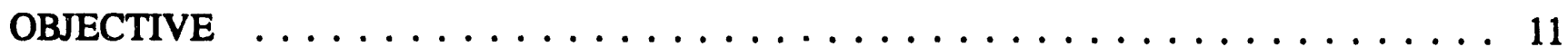

TASK 1: PROJECT PLANNING $\ldots \ldots \ldots \ldots \ldots \ldots \ldots \ldots \ldots \ldots \ldots$

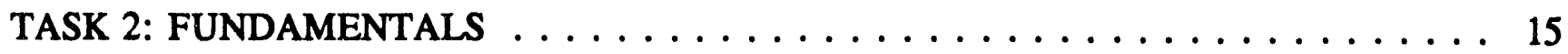

Task 2.1 Surface Forces Measurements . . . . . . . . . . . . . 21

Task 2.2 Determination of Hydrophobic Interaction Parameters . . . . . . 46

Task 2.3 Modeling of Hydrophobic Coagulation . . . . . . . . . . 58

Task 2.4 Measurement of Coagula Size . . . . . . . . . . . . . . . . . . 87

TASK 3: PROCESS DEVELOPMENT . . . . . . . . . . . . . . . . . . . . . . . . 109

Task 3.1 Sample Acquisition . . . . . . . . . . . . . . . . . . 109

Task 3.2 Sample Characterization $\ldots \ldots \ldots \ldots \ldots \ldots \ldots \ldots \ldots \ldots$

Task 3.3 Mixing Studies . . . . . . . . . . . . . . . . . . . . . 122

Task 3.4 Development of Methods for Enhancing the Separation of Coagula . . 128

Task 3.4.1 Addition of Coarse Particles . . . . . . . . . . . 128

Task 3.4.2 Addition of Hydrophobized Magnetite . . . . . . . . 133

Task 3.4.3 Addition of a Hydrocarbon Oil . . . . . . . . . . . 143

Task 3.5 Separator Design and Construction . . . . . . . . . . . . 154

Task 3.6 Bench-Scale Continuous Testing . . . . . . . . . . . . . . . . 160

Task 3.6.1 Low-Sulfur Cool Tests . . . . . . . . . . . . 160

Task 3.6.2 High-Sulfur Coal Tests . . . . . . . . . . . . . . 173

Task 3.7 Process Optimization $\ldots \ldots \ldots \ldots \ldots \ldots \ldots \ldots \ldots$

SUMMARY/CONCLUSIONS . . . . . . . . . . . . . . . . . . . . . . . 190

REFERENCES . . . . . . . . . . . . . . . . . . . . . . . . . . 194

APPENDIX A: SEM-IPS and Centrifugal Washability Data $\ldots \ldots \ldots \ldots \ldots \ldots$ A-1

APPENDIX B: Contour Diagrams $\ldots \ldots \ldots \ldots \ldots \ldots \ldots \ldots \ldots$ B-1 


\section{LIST OF FIGURES}

Figure 1. Schematic illustration of the aggregation mechanisms for various fine particle processes. . . . . . . . . . . . . . 5

Figure 2. Potential energy profile as a function of separation distance as

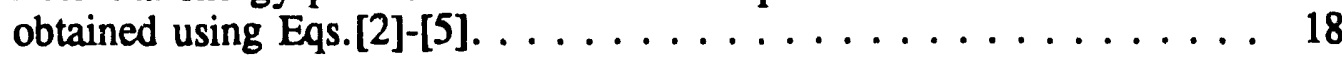

Figure 3. Potential energy profile illustrating the effect of $D_{o}$ on the hydrophobic interaction energy $\left(V_{H}\right) . \ldots \ldots \ldots \ldots$

Figure 4. Potential energy profile showing the effect of $C$ on the hydrophobic interaction energy $\left(V_{H}\right)$ and the total interaction energy $\left(V_{T}\right) \ldots \ldots 22$

Figure 5. Schematic of the Surface Force Apparatus, Mark IV. . . . . . . 23

Figure 6. The effect of dodecylamine hydrochloride (DDA) addition on the surface interaction between two mica plates. . . . . . . . . . 25

Figure 7. Surface pressure $v$ s. head-group area isotherm for a monolayer of

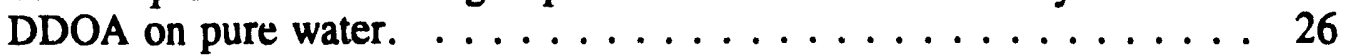

Figure 8. Force - distance curves for DDOA coated mica surfaces. . . . . . . 27

Figure 9. Schematic energy versus distance profiles for wetting films on solids. . . . . . . . . . . . . . . . . . . . 30

Figure 10. Variation of $\mathrm{F}_{d c}, \mathrm{~F}_{\mathrm{dd}}, \mathrm{F}_{\max }$ and $\tau$ as a function of $\mathrm{pH}$, measured with silica surfaces methylated in $10^{-3} \mathrm{M}$ TMCS solution. . . . . . . 33

Figure 11. Variation of $\mathrm{F}_{\mathrm{dc}}, \mathrm{F}_{\mathrm{ed}}$, and $\mathrm{F}_{\max }$ as a function of $\mathrm{KCl}$ concentration (pH about 6.5) measured with silica surfaces methylated in $10^{-4} \mathrm{M}$ TMCS solution. . . . . . . . . . . . . . . . .

Figure 12. Dependence of $\mathrm{F}_{d c}, \mathrm{~F}_{\mathrm{sd}}, \mathrm{F}_{\max }$ and $\tau$ on $\mathrm{W}_{\mathrm{a}}$, obtained in $10^{-3} \mathrm{KCl}$ solution (pH about 6.5). . . . . . . . . . . . . . 37

Figure 13. $\zeta$-potentials of silica particles with and without methylation in $10^{-3}$ $\mathrm{M} \mathrm{KCl}$ solutions as a function of $\mathrm{pH} . \ldots \ldots \ldots \ldots \ldots \ldots$

Figure 14. Surface force profile calculated with Eq.[12] for an air bubble and a silica particle in $10^{-3} \mathrm{M} \mathrm{KCl}$ solution ( $\left.\mathrm{pH} 6.5\right) . \ldots \ldots \ldots \ldots$.

Figure 15. Surface force profile calculated for silica particles methylated in $10^{6}$ $M$ (dashed) \& $10^{-3} \mathrm{M}$ (solid) TMCS solutions, interacting with air bubbles in $10^{-3} \mathrm{M} \mathrm{KCl}$ solution (pH 6.5) . . . . . . . . . . 45

Figure 16. Effect of solution $\mathrm{pH}$ on the coagulation efficiency of fresh and oxidized Elkhorn No. 3 coal. . . . . . . . . . . . . . . . . . 48

Figure 17. Effect of solution pH on the Coagulation Efficiency (as defined by Eq.[2]) of Pittsburgh No. 8 coal. . . . . . . . . . . . . . . .

Figure 18. Zeta potential $(\zeta)$ measured for fresh and oxidized Elkhorn No. 3 coal particles as a function of $\mathrm{pH} . \ldots \ldots \ldots \ldots \ldots \ldots \ldots . \ldots 2$

Figure 19. Effect of $\mathrm{pH}$ on the coagulation efficiency and interaction energy barrier of fresh Elkhon No. 3 coal particles. . . . . . . . . . . . 54

Figure 20. Effect of pH on the coagulation efficiency and interaction energy barrier of partially oxidized Elkhorn No. 3 coal particles. . . . . . . 55

Figure 21. Interaction energy diagram for Elkhom No. 3 coal particles as calculated from the extended DLVO theory. . . . . . . . . 56

Figure 22. Effect of pH on the zeta potential $(\zeta)$ of Pittsburgh No. 8 coal. . . . 57

Figure 23. Calculated total interaction energy $\left(V_{T}\right)$ as a function of particle separation distance for Pittsburgh No. 8 coal. . . . . . . . . . . 
Figure 24. The effect of pH on Coagulation Efficiency and the magnitude (calculated) of the energy barrier for Pittsburgh No. 8 coal.

Figure 25. Kinetic energy as a function of mixing rate for coal samples of two different particle sizes. . . . . . . . . . . . . . . . . . 65

Figure 26. Comparison of collision efficiency for three different values of model parameter $\beta . \ldots \ldots \ldots . \ldots \ldots$

Figure 27. Variation of binding force as a function of aggregate size for three different values of model parameter $k_{1} \ldots \ldots \ldots \ldots 71$

Figure 28. Variation of shear force as a function of aggregate size for three different mixing rates.

Figure 29. Variation of breakage rate constant as a function of aggregate size for different values of model parameters $k_{1}$ and $k_{3} \ldots \ldots \ldots 74$

Figure 30. Variation of breakage rate constant as a function of aggregate size for two different mixing rates. . . . . . . . . . . . . . 76

Figure 31. Schematic diagram of the computer program for simulating coagulation kinetic behavior.

Figure 32. A comparison of aggregate size distributions obtained from computer simulation and experimental measurements at $\mathrm{pH} 8.4$. . . .

Figure 33. Equilibrium aggregate size distributions (predicted) for different values of the Hydrophobic Interaction Parameter, C. . . . . . . . . 84

Figure 34. Equilibrium aggregate size distribution (predicted) for different values of the zeta potential. . . . . . . . . . . . . . . 85

Figure 35. Equilibrium aggregate size distributions (predicted) for different values of the Hamaker constant. . . . . . . . . . . . . . . 86

Figure 36. Equilibrium aggregate size distributions (predicted) as a function of mixing rate. ...................... 88

Figure 37. Coagula top size as a function of mixer agitator speed for the Elkhorn No. 3 coal. . . . . . . . . . . . . . . . . . . . . . . 89

Figure 38. Actual $D_{50}$ vs. Lasentec $D_{50}$ for Elkhorn No. 3 seam coal. . . . . . 90

Figure 39. Actual $D_{50}$ vs. Lasentec $D_{50}$ for Pittsburgh No. 8 seam coal. . . . . . 91

Figure 40. Coagula size distributions for the Elkhorn No. 3 seam coal measured under coagulated and dispersed conditions. . . . . . . . 93

Figure 41. Effect of $\mathrm{pH}$ on the coagula size distribution for the Elkhorn No. 3 seam coal. . . . . . . . . . . . . . . . . . . . . . . 94

Figure 42. Effect of mixing intensity on the coagula size distribution for the Elkhorn No. 3 seam coal. . . . . . . . . . . . . . . . . . . . 96

Figure 43. Effect of $\mathrm{pH}$ and mixing intensity on the mean coagula size for the Elkhorn No. 3 seam coal. . . . . . . . . . . . . . . . . . . . . 97

Figure 44. The effect of initial particle size on the size of coagula produced at an agitation speed of $37 \mathrm{rpm}$ and a pH of $7.5 \ldots \ldots \ldots 100$

Figure 45. The effect of solids concentration on the size of coagula produced at an agitation speed of $25 \mathrm{rpm}$ and a $\mathrm{pH}$ of $7.5 . \ldots \ldots 10 . \ldots 1$

Figure 46. Comparison of the dispersed particle and coagulated particle distributions for a Pittsburgh No. 8 seam coal. . . . . . . . . . . 103

Figure 47. The effect of pH on the coagulation behavior of a Pittsburgh No. 8 seam coal. 
Figure 48. The effect of agitation on the coagulation behavior of a Pittsburgh No. 8 seam coal. . . . . . . . . . . . . . . . . . . . . . 105

Figure 49. Mean coagula size at different mixing intensities $v s$. $\mathrm{pH}$ for a Pittsburgh No. 8 seam coal. . . . . . . . . . . . . . . . . . 106

Figure 50. Effect of kerosene addition and mixing intensity on mean coagula size for Pittsburgh No. 8 coal at pH 7.0. . . . . . . . . . . 108

Figure 51. Effect of grind time on the particle size distribution of Elkhom No. 3 coal. . . . . . . . . . . . . . . . . . . . . . . . 112

Figure 52. Effect of grind time on the particle size distribution of Pittsburgh No. 8 coal. . . . . . . . . . . . . . . . . . . . 113

Figure 53. Grain size distributions for the Elkhorn No. 3 seam coal determined

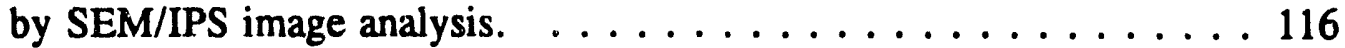

Figure 54. Grain size distributions for the Pittsburgh No. 8 seam coal determined by SEM/IPS analysis. . . . . . . . . . . . . . . 117

Figure 55. Results of centrifugal float-sink tests conducted on the Elkhorn No. 3 seam after grinding for 5,10 and 30 minutes. . . . . . . . . . . 119

Figure 56. Results of centrifugal float-sink tests conducted on the Pittsburgh No. 8 seam after grinding for 5,10 and 30 minutes. . . . . . . . 120

Figure 57. Recovery-rejection curves predicted by SEM-IPS and float-sink techniques for the Elkhorn No. 3 seam afte- 5 and 10 minutes of grinding. . . . . . . . . . . . . . . . . . 123

Figure 58. Recovery-rejection curves predicted by SEM-IPS and float-sink techniques for the Pittsburgh No. 8 seam after 5 and 10 minutes of grinding. . . . . . . . . . . . . . . . . . . . . . 124

Figure 59. Structure and geometry of the mixer used in the coagulation test work. . . . . . . . . . . . . . . . . . . . . . . . 125

Figure 60. Effect of particle kinetic energy $\left(V_{k}\right)$ on the mean coagula size for various cell-impeller configurations at $\mathrm{pH} 7.5 . \ldots \ldots \ldots \ldots \ldots$

Figure 61. Effect of particle kinetic energy $\left(V_{k}\right)$ on the mean coagula size for

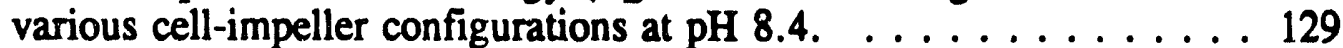

Figure 62. Effect of solids content on the coagula settling behavior for the Elkhorn No. 3 seam coal. . . . . . . . . . . . . . . . . . . . 131

Figure 63. Settling velocity of the coagula as a function of solids content for the Elkhorn No. 3 seam coal. . . . . . . . . . . . . . . . . . . 132

Figure 64. Coagula settling behavior obtained after adding $200 \times 270$ mesh Buller seam to micronized Elkhorn No. 3 seam coal. . . . . . . . . . 134

Figure 65. Coagula settling behavior obtained after adding 270x325 mesh Buller seam to micronized Elkhorn No. 3 seam coal. . . . . . . . . 135

Figure 66. Coagula settling behavior obtained after adding 325 400 mesh Buller seam to micronized Elkhorn No. 3 seam coal. . . . . . . . . . 136

Figuie 67. Coagula settling velocity obtained after adding various size fractions of Buller coal to the Elkhom No. 3 seam coal. . . . . . . . . . . 137

Figure 68. Coagula settling behavior obtained after adding $200 \times 270$ mesh magnetite to micronized Elkhorn No. 3 seam coal. . . . . . . . . . . 139

Figure 69. Coagula settling behavior obtained after adding $270 \times 325$ mesh magnetite to micronized Elkhom No. 3 seam coal. 
Figure 70. Coagula settling behavior obtained after adding $325 \times 400$ mesh magnetite to micronized Elkhorn No. 3 seam coal. . . . . . . . . . . 141

Figure 71. Coagula settling velocity obtained after adding various size fractions of magnetite to the Elkhom No. 3 seam coal. . . . . . . . . . . . . 142

Figure 72. Effect of kerosene addition and mixing intensity on mean coagula size for Elkhorn No. 3 seam coal. . . . . . . . . . . . . . . . 145

Figure 73. Effect of diesel addition and mixing intensity on mean coagula size for Elkhorn No. 3 seam coal. . . . . . . . . . . . . . . . . . . . . . 146

Figure 74. Effect of coal-oil addition and mixing intensity on mean coagula size for Elkhorn No. 3 seam coal. . . . . . . . . . . . . . . . . . . . 147

Figure 75. Effect of kerosene additions and mixing intensity on mean coagula size for Elkhorn No. 3 seam coal at pH 7.45. . . . . . . . . . . . . 148

Figure 76. Effect of diesel addition and mixing intensity on mean coagula size for Elkhorn No. 3 seam coal at pH 7.45. . . . . . . . . . . . . . . 149

Figure 77. Effect of kerosene additions on coagula settling rates for Elkhorn No. 3 seam coal at pH 7. . . . . . . . . . . . . . . . . . . 151

Figure 78. Effect of diesel additions on coagula settling rates for Elkhorn No. 3 seam coal at $\mathrm{pH} 7 . \ldots \ldots \ldots \ldots . \ldots \ldots \ldots$

Figure 79. Effect of coal oil additions on coagula settling rates for Elkhom No. 3 seam coal at pH 7. . . . . . . . . . . . . . . . . . . . 153

Figure 80. Effect of kerosene additions and pH on coagula settling rates for a partially oxidized sample of Elkhorn No. 3 seam coal. . . . . . . . . . 155

Figure 81. Schematic of the sedimentation tank to be used for separating coagulated coal particles from dispersed mineral matter. . . . . . . . . 158

Figure 82. Schematic of the rotating screen to be used for separating coagulated coal particles from dispersed mineral matter. . . . . . . . . 159

Figure 83. Coal recovery and product ash content vs. retention time in the continuous, bench-scale treatment of an Elkhorn No. 3 seam coal. . . 166

Figure 84. The effect of solids concentration on the separation performance of the continuous SHC unit when processing an Elkhom No. 3 seam coal. . . . . . . . . . . . . . . . . . . . . . . . . . . . . . . . . . 169

Figure 85. Process response to changes in Mudline and Feed Point at high Feed Rates. . . . . . . . . . . . . . . . . . . . . . . . . . . . . 182

Figure 86. Process response to changes in Mudline and Feed Point at low Feed Rates. . . . . . . . . . . . . . . . . . . . . . . . . . . . 183

Figure 87. Process Response to changes in Feed Rate and Solids Concentration at high Mudlines and Feed Points. . . . . . . . . . . . . . . . . . 184

Figure 88. Process response to changes in Feed Rate and Solids Concentration at low Mudlines and Feed Points. . . . . . . . . . . . . . . . 185 


\section{LIST OF TABLES}

Table I. Comparison of force barriers calculated using the conventional heterocoagulation theory and experimental

values determined as a function of $\mathrm{pH} \ldots \ldots . . . \ldots 41$

Table II. The values of the hydrophobic force constant calculated from

the measured force barrier as a function of surface

hydrophobicity.

Table III. Results illustrating the effect of initial particle size on the mean coagula size over a range of $\mathrm{pH}$ values and agitation speeds. . . . . . . . . . . . . . . . . . . . . . 98

Table IV. Results of sulfur forms, total sulfur, proximate, and calorimetry analyses conducted on Pittsburgh No. 8 and Elkhorn No. 3 coal samples.

Table V. Product size distribution parameters for attrition grinding of Pittsburgh No. 8 and Elkhorn No. 3 coal samples. . . . . . . . . . 111

Table VI. Weight distribution of mineral matter as a function of grinding time for an Elkhorn No. 3 coal seam sample. . . . . . . . . 114

Table VII. Weight distribution of mineral matter as a function of grinding time for a Pittsburgh No. 8 coal seam sample. . . . . . . . 115

Table VIII. Cell configurations utilized in the SHC mixing studies.

Table IX. Tabulated results illustrating the effect of feed solids content on the separation performance of a continuous sedimentation tank (thickener).

Table X.

Tabulated results illustrating the effect of feed rate on the separation performance of a continuous sedimentation tank (thickener).

Table XI. Tabulated results illustrating the effect of wash water rate on the separation performance of a continuous sedimentation tank.

Table XII. Tabulated results illustrating the effect of feed rate and feed solids content on the separation performance of a continuously rotating drum screen. . . . . . . . . . . . . . . . . . . 163

Table XIII. Results illustrating the effect of feed rate on the separation performance of the SHC process using an 11-inch diameter continuous sedimentation tank. . . . . . . . . . . . . . 168

Table XIV. Results illustrating the effect of pH on the efficient separation of selectively coagulated Elkhom No. 3 coal from its associated mineral matter using an 11-inch diameter continuous sedimentation tank.

Table XV. Results illustrating the effect of particle size on the separation performance of the SHC process using an 11-inch diameter continuous sedimentation tank.

Table XVI. Results showing the effect that dispersant and/or chelating agent additions have on the separation performance of the SHC process. . . . . . . . . . . . . . . . . . . . . 172

Table XVII. Results showing the effect of dispersant additions on the separation performance of the SHC process. 
Table XVIII. Results showing the effect of chelating agent additions on the separation performance of the SHC process. . . . . . . . . . . . 173

Table XIX. Results illustrating the effect of $\mathrm{pH}$ on the separation of selectively coagulated Pittsburgh No. 8 coal from its associated mineral matter using an 11-inch diameter continuous sedimentation tank. . . . . . . . . . . . . . . . . 174

Table XX. The Box-Behnken test design for the continuous SHC process utilizing a sedimentation tank for separating the selectively cosgulated coal from its associated mineral matter. . . . . . . . . . 176

Table XXI. Metallurgical responses for the various tests conducted in accordance with the Box-Behnken experimental design. . . . . . . . 177

Table XXII. A comparison between the predicted separation efficiency values obtained from linear, quadratic and cubic models and the actual experimental values.

Table XXIII. List of variables/coefficients for the quadratic models for product ash, recovery, and separation efficiency for the $S H C$ sedimentation tank. . . . . . . . . . . . . . . . . 186

Table XXIV. Results comparing the predicted response values and actual test values obtained using a continuous sedimentation tank. . . . . . . 187

Table XXV. Experimental results for tests conducted at the calculated optimum operating point for the SHC sedimentation tank. . . . . . . . . 189 


\section{INTRODUCTION}

Recent studies carried out at the Virginia Center for Coal and Minerals Processing (VCCMP) have resulted in the development of a novel agglomeration process for upgrading ultrafine coal. This process, which is known as selective hydrophobic coagulation (SHC), is based on the new finding that hydrophobic coal particles can be selectively coagulated in the presence of dispersed mineral matter. The driving force for the coagulation is believed to be the hydrophobic structural force, which has been recognized only recently in the scientific community. In most cases, selective separations can be achieved using simple $\mathrm{pH}$ control to disperse the mineral matter, followed by recovery of the coal coagula using techniques that take advantage of the size enlargement.

Background: The control of the stability of colloidal dispersions is practiced in a variety of different industries and has interested chemists and engineers for many years. Biologists, for example, are concerned with aggregation of blood platelets (agglutination) in blood clotting. In the paper industry, engineers must keep the pigments in aqueous suspension. The coal-water mixture (CWM) technology in the coal industry hinges on keeping particles in suspension. In the mineral industry, engineers are concerned with improving the rate of thickening and filtration by controlling the state of aggregation.

A colloidal dispersion is said to be unstable when particles agglomerate and settle at the bottom of the container. This agglomeration can be induced by adding electrolytes, which is referred to as electrolytic coagulation, or by adding synthetic or natural flocculants, which is called flocculation. La Mer and Healy $(1963,1966)$ strongly advocated that the distinction between coagulation and flocculation be made on the basis of the different mechanisms involved. 
Coagulation refers to the process of aggregation in which particles are driven together by the mechanism generally known as the DLVO theory (Derjaguin and Landau, 1943; Verwey and Overbeek, 1948). According to this theory, which will be discussed later in further detail, electrolytes added to the suspension can reduce the electrostatic repulsive force between two particles of the same kind, so that they can approach each other closely enough for the London-Van der Waals' force to cause aggregation. Since this process is induced by the addition of electrolytes, the process is often referred to as electrolytic coagulation. The amount of electrolytes needed to induce the coagulation, i.e., the critical coagulation concentration $(C C C)$, decreases as the cube of the valence of the counter ions of the electrolyte, as embodied in the well-known Schulze-Hardy rule. Thus, divalent ions such as $\mathrm{Ca}^{2+}$ and trivalent ions such as $\mathrm{Al}^{3+}$ are used effectively to coagulate negatively-charged particles.

Maynard et al. (1968) developed a process of selectively coagulating anatase $\left(\mathrm{TiO}_{2}\right)$ impurities from kaolin clay, in which a peptizing agent such as sodium hexametaphosphate or sodium silicate was added in excess of what is needed to obtain the minimum viscosity. If the amount of reagent used was at least $100 \%$ beyond the optimum needed to achieve minimum viscosity, they found that the anatase coagulated while the clay remained in suspension. It is likely that the excess electrolyte compressed the electrical double layer to the extent that the zeta-potential of the anatase was reduced to below that of the clay. According to Pugh and Kitchener (1971), a 20-30 mV difference in the zeta-potentials is ideal for selective coagulation.

Flocculation refers to the process in which particles are brought together by soluble macromolecules such as starches, alginates, gums and a variety of synthetic polymers, by a bridging mechanism. Flocculation is characteristically much more rapid 
than coagulation. Flocs are generally much larger than coagula and have a loose, open structure.

Warren $(1975,1981,1982)$ coined the term, shear flocculation, denoting the means by which particles hydrophobized by proper surfactant coating can be aggregated by subjecting the suspension to high-shear agitation. He noted that "shear coagulation occurs only when the energy of the impact of colliding particles is sufficient to overcome an as-yet undefined energy barrier preventing their spontaneous coalescence." This theory is based on the conjecture that high-shear agitation can indeed provide sufficient kinetic energy to overcome the energy barrier.

The DLVO theory suggests that coagulation of particles is controlled by two competing energies, a repulsive electrostatic interaction energy $\left(V_{R}\right)$ and an attractive London-van der Waals dispersion energy $\left(V_{A}\right)$. The calculation of the sum of these two components, for $5-\mu \mathrm{m}$ coal particles in $10^{-3}$ moles $/ 1 \mathrm{KCl}$ solution at $\mathrm{pH} 8.9$, shows that the total interaction energy, $\left(\mathrm{V}_{\mathrm{T}}\right)$, reaches a maximum $\left(\mathrm{V}_{\max }\right)$ of about $2,924 k T$ at a distance of approximately $1.2 \mathrm{~nm}$ from the particle surface. According to the theory of shear flocculation described above, a high-shear agitation that can provide a kinetic energy exceeding $V_{\max }$ must be applied. $X u$ and Yoon (1989) showed, however, that no more than $60 k T$ of kinetic energy provided by means of moderate mixing was sufficient to bring about the aggregation of coal. In this regard, the term shear flocculation is a misnomer since there is no need to provide high-shear agitation to overcome the energy barrier.

In fact, the kinetic energy provided by mixing is not nearly enough to overcome the energy barriers predicted by the DLVO theory. Warren $(1977,1984)$ incorrectly estimated the kinetic energy provided by mixing for $1-\mu \mathrm{m}$ particles to be about $1,000 \mathrm{kT}$. 
This calculation was made assuming a value of $5 \mathrm{~cm} / \mathrm{sec}$ for the velocity of particles in stirred tanks. However, the kinetic energy calculated for a $1-\mu \mathrm{m}$ scheelite particle (S.G. $=6.0)$ at $1,700 \mathrm{rpm}$ using the method described by Xu (1990) is only $0.45 \mathrm{kT}$.

Warren's calculation was based on the absolute velocity, which is not a realistic assumption in calculating the kinetic energies of colliding particles in an aqueous medium. Chia and Somasundaran (1983) used a value of $0.22 \mathrm{~cm} / \mathrm{sec}$ as the relative velocity of the 1.1 $\mu \mathrm{m}$ anatase particles, and obtained $6.5 \mathrm{kT}$ as the kinetic energy for collision. The major role of the high-shear agitation employed in Warren's experiments may be to increase the rate of aggregation, at least for very small particles.

On the other hand, the process described by Warren may be appropriately referred to as flocculation, in that the hydrocarbon tails of the adsorbed oleate molecules are actually touching, overlapping or intermingling with each other (Warren, 1975, 1s.81). This is similar in form to the process of polymer flocculation (Figure 1). The close association of the hydrocarbon tails will give rise to a significant negative free energy change, as is the case with micellization. Knowing that the free energy of micellization is in the range of -0.9 to $1.2 \mathrm{kT} / \mathrm{mole}$ of $\mathrm{CH}_{2}$ group, Warren (1975) estimated the attractive energy due to the association of hydrocarbons to be on the order of 1,000 $10,000 k \mathrm{~T}$ for a $1-\mu \mathrm{m}$ particle. This large energy gained from the hydrophobic association may provide an explanation for the ubservation that the aggregates formed in his experiments with oleate-coated scheelite could withstand the high-shear agitation.

Apparently, a long chain surfactant coating is necessary for shear flocculation to occur. While sodium oleate and sodium laureate coatings on cassiterite $\left(\mathrm{SnO}_{2}\right)$ produced aggregation, styrene phosphoric acids could not, despite the fact that the latter could make the mineral surface hydrophobic enough to stick to the surface of air bubbles (Warren, 1982). It was explained by Warren that "the short hydrocarbon chain may be 


Name

Figure 1. Schematic illustration of the aggregation mechanisms for various fine particle processes. 
too short to give a significant energy of hydrophobic association when the chains on colliding particles overlap."

Koh et al. (1985) proposed that during the process of shear flocculation, particles are also held together by a type of "liquid bridge" formed at the point of contact. This bridge is formed by the capillary condensation of undissociated oleic acid. This mechanism further substantiates the idea that surfactant-coated particles are held together by a bridging mechanism in a manner similar to polymer flocculation.

The bridging mechanism also operates in oil agglomeration (Figure 1). When a small amount of oil and intense agitation are used, e.g., less than $5 \%$ for particles less than $0.5 \mathrm{~mm}$, particles form unconsolidated flocs through pendular bridges in a twodimensional network. When such flocs are recovered on a screen, the yield is poor because of the poor integrity of the agglomerates. The flocs also tend to trap unwanted hydrophilic mineral matter and water which do not drain away readily. For this reason, the volume of settled agglomerates increases with increasing oil addition in this pendular flocculation region (Drzymala et al., 1986). With a larger amount of oil, e.g., 5-15\%, some of the pendular bridges coalesce to form a three-dimensional network. In this funicular region, the number of oil junctions per particle increases with increasing amounts of added oil, which results in a decrease in the amount of mineral matter and water entrapped. With a further increase in oil addition, all the voids are filled with oil and spherical agglomerates or pellets are formed. In this capillary wetting region, the entrapment of mineral matter and water becomes minimal and, therefore, the process becomes most efficient in terms of ash rejection and dewatering characteristics. For typical fine coals of less than $0.5 \mathrm{~mm}$, this capillary wetting region is reached in the 15$20 \%$ range (Capes, 1989), while for micronized coals it is obtained in the $45-55 \%$ range (Keller, 1984). This is, perhaps, the main reason why the Otisca-T process is so 
successful in producing ultraclean coals. In this process, one can afford to use such a large quantity of oil (pentane) since most of it is recovered. This is not the case with conventional oil agglomeration processes that use "disposable" oil since economics dictate that oil consumption be kept to a minimum.

Even for those cases involving use of recoverable oils, the cost of the oil recuvery system amounts to a significant portion of the total process cost. It would be of interest, therefore, to develop an agglomeration process where the amount of oil is reduced substantially. It has been reported that oil consumption can be reduced to as little as $1 \%$ by using more efficient mixers and by using the froth flotation technique to separate the microflocs from the dispersed mineral phase (Capes, 1989). An added benefit of minimizing the oil consumption is that pyrite rejection improves proportionately. It should be noted, however, that the low dosage oil agglomeration process is essentially no different from the aggregate flotation process advocated by the Illinois State Geological Survey (Read et al., 1987) and the Technical University of Nova Scotia (Wojcik and Al Taweel, 1984). If the aggregate flotation process is no better than advanced froth flotation processes, which require oil in tie range of $0-0.01 \%$, then neither would be the low dosage oil agglomeration process using flotation.

Recent studies carried out at VCCMP have demonstrated that hydrophobic materials such as coal and methylated silica can be coagulated spontaneously without the addition of electrolyte or hydrocarbon agglomerant. This phenomenon cannot be explained by the classical DLVO theory, which predicts a very large energy barrier ( $3,000 \mathrm{kT}$ ). However, when the classical DLVO theory is extended to include the hydrophobic interaction energy, $\left(V_{H}\right)$, the energy barrier is reduced to the level of approximately $60 k \mathrm{~T}$, which is in line with experimental observation. 
The existence of $V_{H}$ has been proved experimentally by many investigators (Israelachvili and Pashley, 1982, 1984; Claesson et al., 1986; Christenson, 1988; Rabinovich and Derjaguin, 1987) using direct force measurement devices. However, there is still a controversy as to the origin of this non-DLVO energy. Many investigators (Israelachvili, 1978; Israelachvili and Pashley, 1982, 1984; Claesson et al., 1986; Rabinovich and Deriaguin, 1988; Derjaguin et al., 1978) consider it entropic in origin, arising mainly from the configurational rearrangement of water molecules in the vicinity of hydrophobic surfaces, while others believe that it is due to phase changes in the interlayer between two surfaces in close proximity (Claesson, 1987; Pashley, 1981; Christenson et al., 1987) or to anomalous polarization of water molecules near hydrophobic surfaces.

When two or more hydrophobic particles are brought together by virtue of the hydrophobic interaction force $\left(V_{H}\right)$ to form aggregates, this phenomenon should be referred to as hydrophobic coagulation. The term coagulation is appropriate here because aggregation does not depend on the addition of bridging agents, such as organic polymers, that are utilized in flocculation. For the case of electrolytic coagulation, particles are considered to be separated by about $0.75 \mathrm{~nm}$ (Warren, 1981; Frens and Overbeek, 1972; Firth and Hunter, 1976), while the separation distance may be considerably shorter for hydrophobic coagulation. This is borne out by considering that the hydrophobic interaction energy is generally larger than the dispersion interaction energy, although the absolute magnitude will depend on the degree of hydrophobicity of the particles involved.

Since there are no bridging mechanisms involved, the process of hydrophobic coagulation requires no reagent. The only reagents that may be needed are those that would help prevent the associated mineral matter from electrolytically coagulating. 
Dispersion of the mineral matter can often be achieved by simple $\mathrm{pH}$ control. If a coal is oxidized, a small addition of reagents to enhance the hydrophobicity may be needed. For most unoxidized U.S. coals, however, no such reagents are necessary. Furthermore, there is no need to employ high-shear agitation, since the energy barrier is relatively low. This feature also distinguishes hydrophobic coagulation from shear flocculation. Agitation may be useful to increase the coagulation rate by increasing the frequency of collision between particles, although increased agitation may also lead to an higher coagula breakage rates. Another advantage of the hydrophobic coagulation process may be that since no hydrocarbon oils are used, separation of pyritic sulfur is easier than with those processes using large amounts of oil.

However, there is a drawback to the hydrophobic coagulation process. Preliminary tests conducted at VCCMP indicate that the coagula formed by hydrophobic coagulation are much weaker than the flocs formed by traditional oil agglomeration processes. Therefore, special techniques are required to separate the hydrophobic coagula from the dispersed mineral matter. 


\section{OBJECTIVE}

The overall objective of the current project was to develop an economical method of producing low-ash, low-sulfur coals using the selective hydrophobic coagulation (SHC) process. The work was subdivided into three tasks: 1) project planning; 2) studies of the

fundamental mechanism of the selective hydrophobic coagulation process parameters that affect the process of separating coal from both the ash-forming minerals and pyritic sulfur; and 3) bench-scale process development which would establish the best possible method of separating the coagula from the dispersed mineral matter. 


\section{TASK 1: PROJECT PLANNING}

The detailed work plan for the project was forwarded to the DOE on November 29, 1990, and was approved shortly thereafter. The plan consisted of 1) an itemization of the work, which included detailed descriptions of the technical approach/methods to be used to achieve the objectives of the contract, and 2) a detailed project schedule, including a manpower schedule by work breakdown structure element. 


\section{TASK 2: FUNDAMENTALS}

The classical DLVO theory has been used extensively to describe the stability of lyophobic colloids. This theory states that the potential energy $\left(V_{T}\right)$ of interacting particles is given by the sum of the repulsive electrostatic interaction energy $\left(V_{R}\right)$ and the attractive London-van der Waals dispersion energy $\left(V_{A}\right)$ as follows:

$$
V_{T}=V_{R}+V_{A},
$$

in which

$$
V_{R}=\frac{\varepsilon a \psi_{d}^{2}}{2} \ln [1+\exp (-\mathrm{k} H)],
$$

and

$$
V_{A}=-\frac{a A_{131}}{12 H},
$$

where $A_{13 t}$ is the Hamaker constant (Hamaker, 1937) for two spheres of 1 in a medium 3, $a$ the radius, $H$ the separation distance, $\varepsilon$ the dielectric constant, $\psi_{d}$ the Stern potential, and $x$ is the Debye reciprocal length. The $\zeta$-potential is commonly used as an estimate of the Stern potential. Methods for determining the other parameters in Eqs. [2] and [3] have been described in literature (Fowkes, 1964; Bargeman and Vader, 1972; Derjaguin, 1934).

While the classical DLVO theory is useful for describing the stability of moderately hydrophilic and weakly hydrophobic particles, it fails with very hydrophobic particles. Xu and Yoon $(1989,1990)$ have shown that unoxidized bituminous coals can be coagulated under conditions of high $\zeta$-potentials, indicating that there may be an additional attractive energy other than $V_{A}$. Thus, the classical DLVO theory has been extended as follows:

$$
V_{T}=V_{R}+V_{A}+V_{H},
$$


in which $V_{H}$ represents the additional attractive energy associated with hydrophobic particles and can be described using the following equation:

$$
V_{H}=\frac{a C D_{0}}{2} \exp \left(-\frac{H}{D_{0}}\right),
$$

in which $C$ is the hydrophobic interaction constant and $D_{0}$ is the characteristic decay length. Eq. [5] was originally proposed by Israelachvili and Pashley (1982) based on direct surface force measurements. This equation has also been theoretically derived from a mean-field theory based upon the idea that the hydrophobic interaction force is a result of structural changes in the thin water film separating two hydrophobic surfaces (Xu and Yoon, 1990; Eriksson et al., 1989)

There are several hypotheses suggested in literature on the nature of the attractive hydrophobic interaction energy (Xu and Yoon, 1990). One of the theories states that the long range attraction between two hydrophobic solids is a result of induced electrostatic fluctuations between neutral bodies, which is the same origin as the classical van der Waals energy (Attard, 1989). However, this theory has been found to predict interaction energies inat are too small to explain the experimentally observed hydrophobic attraction. A more widely accepted theory accounts for the non-DLVO attractive interaction energy by considering the structure of the water molecules at the surface of hydrophobic materials. It has been postulated that upon the interaction of two hydrophobic surfaces, the water molecules align themselves parallel to the solid surface resulting in a decrease in the degrees of freedom of the water molecules. Thus, the thin water film between two interacting hydrophobic surfaces is thermodynamically unstable. As two hydrophobic surfaces are brought together, the structured water molecules are released into the less structured bulk water, resulting in an increase in entropy to the systern, which is a thermodynamically favorable process. Based on this, it has been proposed that the 
attractive hydrophobic interaction energy is entropy-driven (Israelachvili, 1985; Xu and Yoon, 1989).

Based on the extended DLVO theory embodied in Eqs. [4] and [5], one can calculate each component (i.e., $V_{R}, V_{A}$, and $V_{H}$ ) of the total interaction energy, $V_{T}$, as a function of separation distance, $H$. An example of such a calculation is shown in Figure 2. These calculations were made assuming that $C=1.2 \mathrm{~mJ} / \mathrm{m}^{2}$ and that $D_{o}=10.3 \mathrm{~nm}$, which have been reported to be the case with strongly hydrophobic particles. Figure 2 shows that the attractive hydrophobic interaction energy $\left(V_{H}\right)$ and the repulsive electrostatic interaction energy $\left(V_{R}\right)$ play the dominate role in determining the stability of hydrophobic solids. The attractive dispersion interaction energy, $V_{A}$, is a relatively short range force and thus plays a minor role.

The value of the characteristic decay length $\left(D_{0}\right)$ indicates how quickly the hydrophobic energy decays with separation distance, $\boldsymbol{H}$. For very hydrophobic surfaces, $D_{o}$ values have been found to be in the range of 12 to $16 \mathrm{~nm}$ (Christenson et al., 1989; Rabinovich and Derjaguin, 1988; Claesson and Christenson, 1988). In order to illustrate the effect of the decay length on stability predictions, $V_{T}$ was calculated and plotted with $V_{H}$ as a function of $H$ using Eqs. [2]-[5] for three different $D_{o}$ values. As shown in Figure 3, larger $D_{o}$ values yield a hydrophobic interaction energy that has a longer range of influence on $V_{r}$. As a result, the total interaction energy is lower for high values of $T A S D_{o}$.

$\mathrm{Xu}$ and Yoon (1990) found that the value of the hydrophobic constant, $C$, is dependent upon the degree of surface hydrophobicity. They came to this conclusion after studying the surface characteristics and coagulation behavior of fresh and oxidized bituminous coal and silica that was methylated with varying concentrations of 


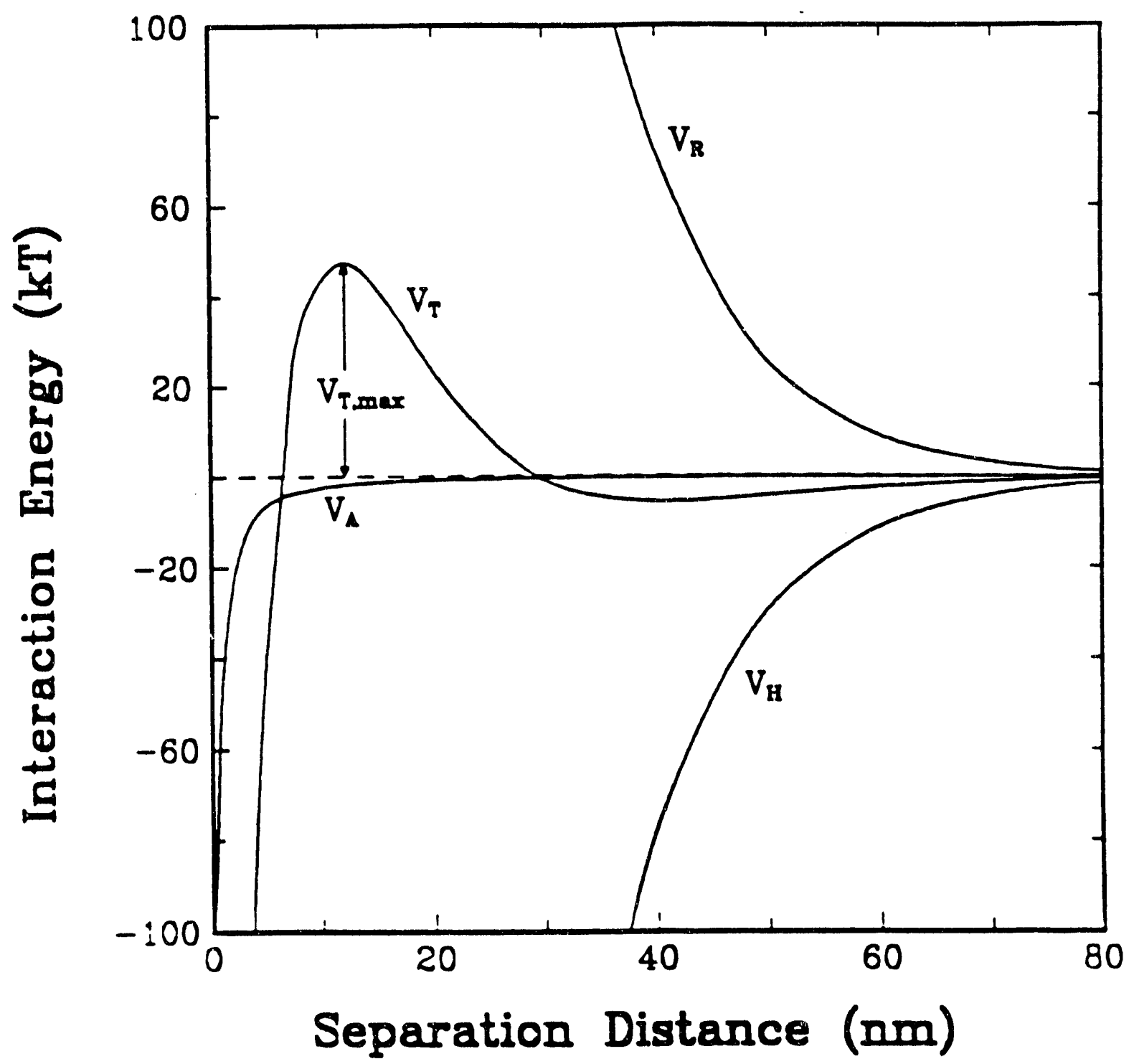

Figure 2. Potential energy profile as a function of separation distance as obtained using Eqs.[2]-[5]. 


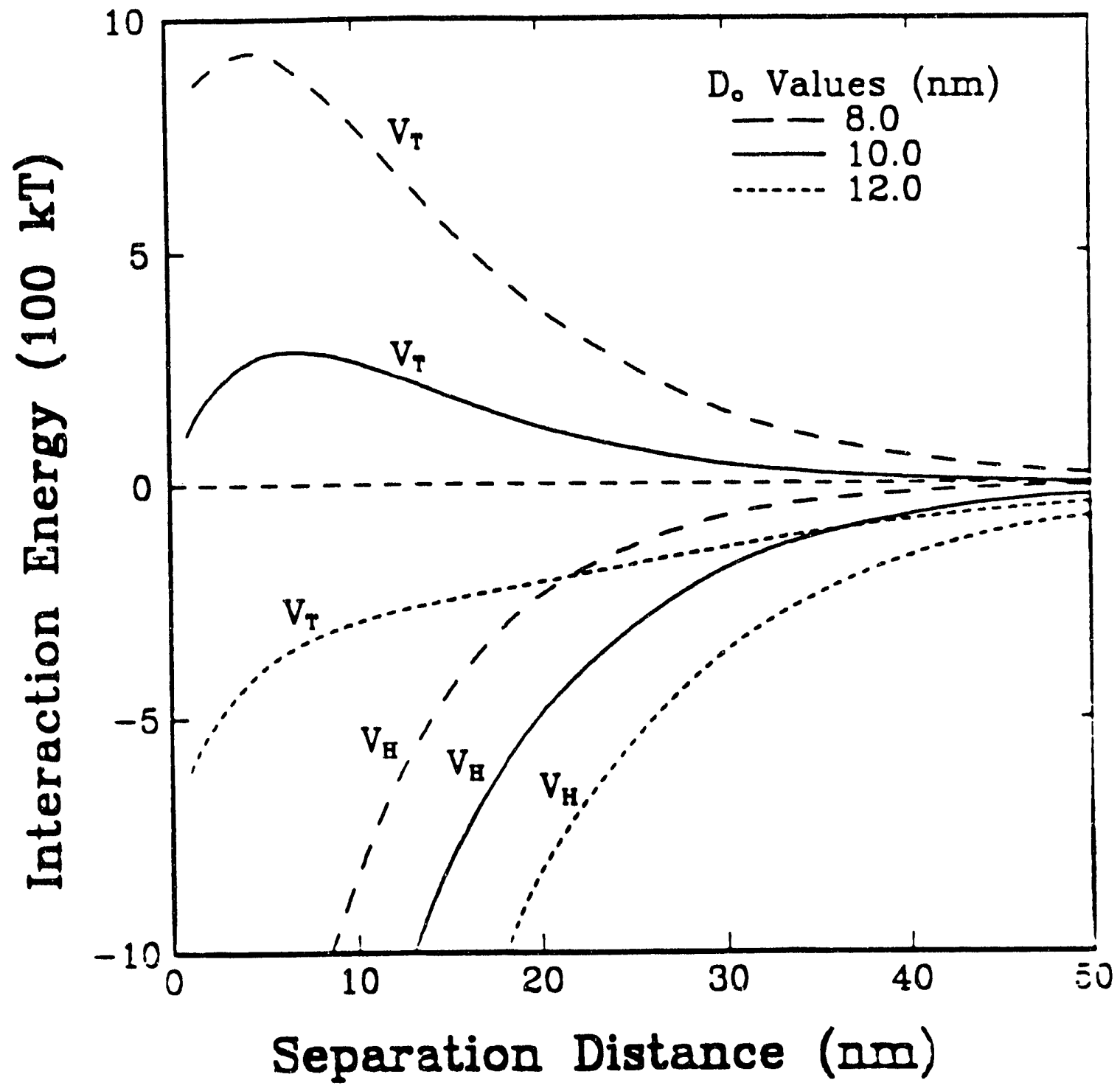

Figure 3. Potential energy profile illustrating the effect of $D_{o}$ on the hydrophobic interaction energy $\left(V_{H}\right)$. 
trimethylchlorosilane (TMCS) to obtain different degrees of hydrophobicity. In their investigation, they derived an expression whereby the non-dispersion component of the work of adhesion, $W_{a}^{\text {md }}$, can be used to determine the value of $C$ :

$$
C=\frac{C_{m}}{1+\exp \left[b\left(W_{a}^{\text {dd }}-K\right)\right.} \mathrm{m} / / \mathrm{m}^{2},
$$

in which $C_{m}(=-1.89), b(=0.49)$ and $K(=34.8)$ are empirical-fitting parameters. Assuming that $D_{o}=10.3 \mathrm{~nm}, \mathrm{Xu}$ and Yoon (1990) determined the values of these fitting parameters by conducting coagulation experiments with particle suspensions of different hydrophobicities.

For a given solid, $W_{a}^{\text {nd }}$ can be determined using the following relationship:

$$
W_{a}^{n d}=\gamma_{w}\left(1+\cos \theta_{w}\right)-2 \sqrt{\gamma_{s}^{d} \gamma_{w}^{d}},
$$

in which $\theta_{w}$ is the water contact angle, $\gamma_{w}\left(72.8 \mathrm{~mJ} / \mathrm{m}^{2}\right)$ is the surface free energy of water, and $\gamma_{1}^{d}$ and $\gamma_{w}^{d}\left(=22.8 \mathrm{~mJ} / \mathrm{m}^{2}\right)$ are the dispersion components of the surface free energy of the solid and water, respectively. Methylene iodide contact angle measurements can be used to determine $\gamma_{i}^{d}$ according to the following equation:

$$
\gamma_{s}^{d}=\left[\frac{\left(1+\cos \theta_{m}\right) \gamma_{m}}{2 \sqrt{\gamma_{m}^{d}}}\right]^{p},
$$

in which $\theta_{\mathrm{m}}$ is the contact angle, $\gamma_{\mathrm{m}}$ is the surface free energy of the methylene iodide $\left(50.8 \mathrm{~mJ} / \mathrm{m}^{2}\right)$, and $\gamma_{\mathrm{m}}^{d}$ is its dispersion component $\left(48.5 \mathrm{~mJ} / \mathrm{m}^{2}\right)$. Thus, by performing methylene iodide and water contact angle measurements, the non-dispersion component of the work of adhesion and, hence, the hydrophobic constant can be easily determined for a particular hydrophobic solid. Once the value of $C$ is known, the hydrophobic interaction energy can be calculated as a function of separation distance using Eq. [4]. 
Particles that are strongly hydrophobic have a low $W_{a}^{\text {md }}$ value and, according to

Eq. [6], a high absolute value for $C$. One can conclude from Eq. [5] that a high negative $C$ value results in a very strong attractive hydrophobic interaction energy. This is illustrated in Figure 4 where $V_{T}$ and $V_{H}$ are plotted as a function of separation distance for $C$ values of $0.5,1.0$, and $1.5 \mathrm{~mJ} / \mathrm{m}^{2}$. This figure shows the increase in the dominating role of $V_{H}$ as the $C$ value or surface hydrophobicity increases.

The results of the work presented in this section showed that it is important to know appropriate values of $C$ and $D_{0}$ in estimating the magnitude of $V_{H}$. For this reason, direct surface force measurements have been conducted in the present work using a model hydrophobic system. The results of these measurements are presented in the following sections.

\section{Task 2.1 Surface Forces Measurements}

a) Direct Surface Force Measurements

The Surface Force Apparatus, Mark IV (Figure 5), provides a direct means for measuring the attachment and detachment forces between two solid surfaces as a function of separation distance. Basically, the apparatus consists of three elements: an optical interferometry system that provides a convenient method to measure surface separation (to a resolution of about $0.2 \mathrm{~nm}$ ); a weak spring to which one of the surfaces is mounted and whose deflection is related by Hookes' law to the force between the two surfaces; and a piezoelectric actuator that controls the separation of the two surfaces.

Because the surface force apparatus was new to the center, a preliminary set of tests was conducted to establish that the device was working satisfactorily. In this work, the effect of dodecylamine (DDA) additions on the force-distance relationships between 


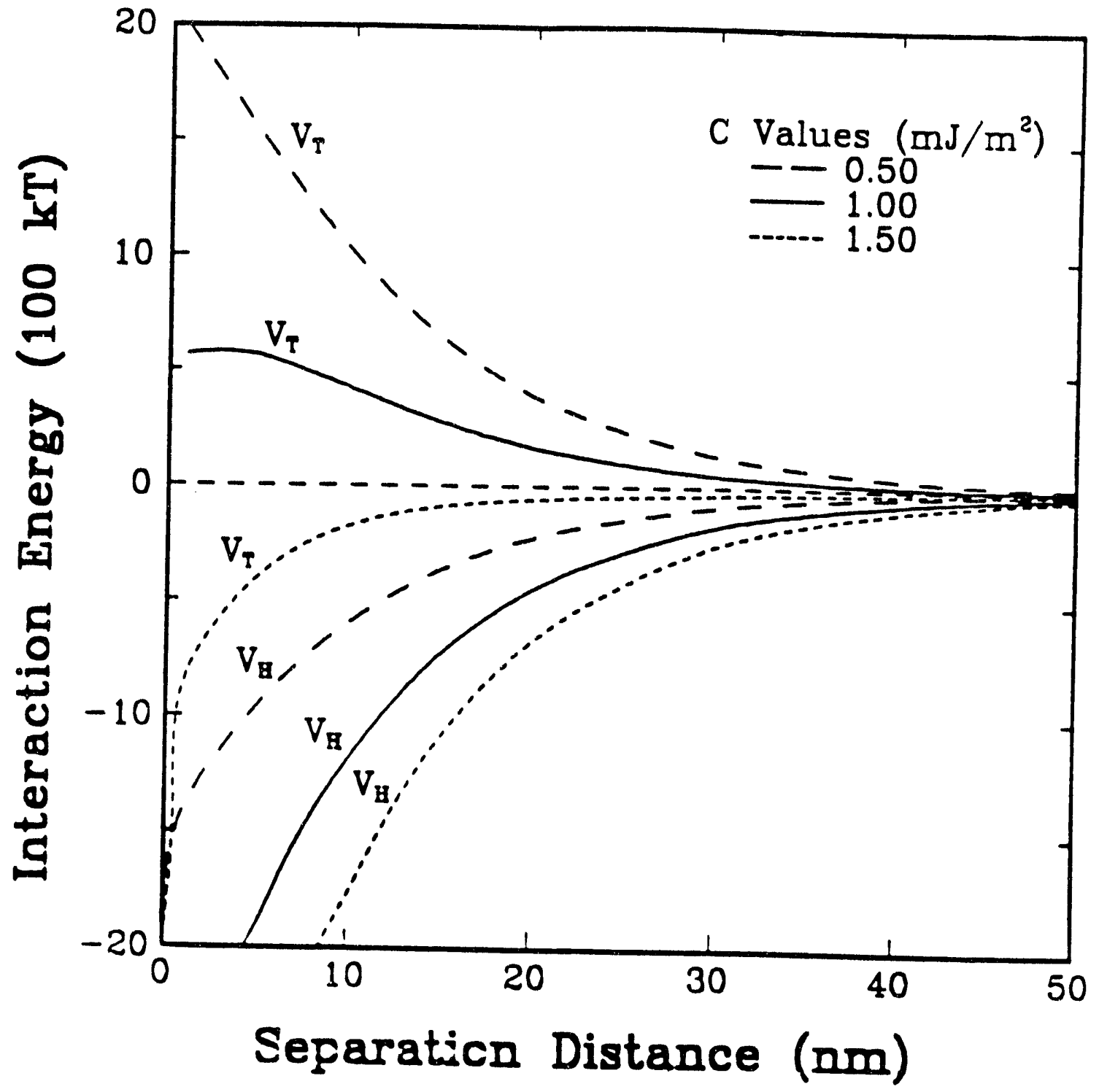

Figure 4. Potential energy profile showing the effect of $C$ on the hydrophobic interaction energy $\left(V_{H}\right)$ and the total interaction energy $\left(V_{T}\right)$. 


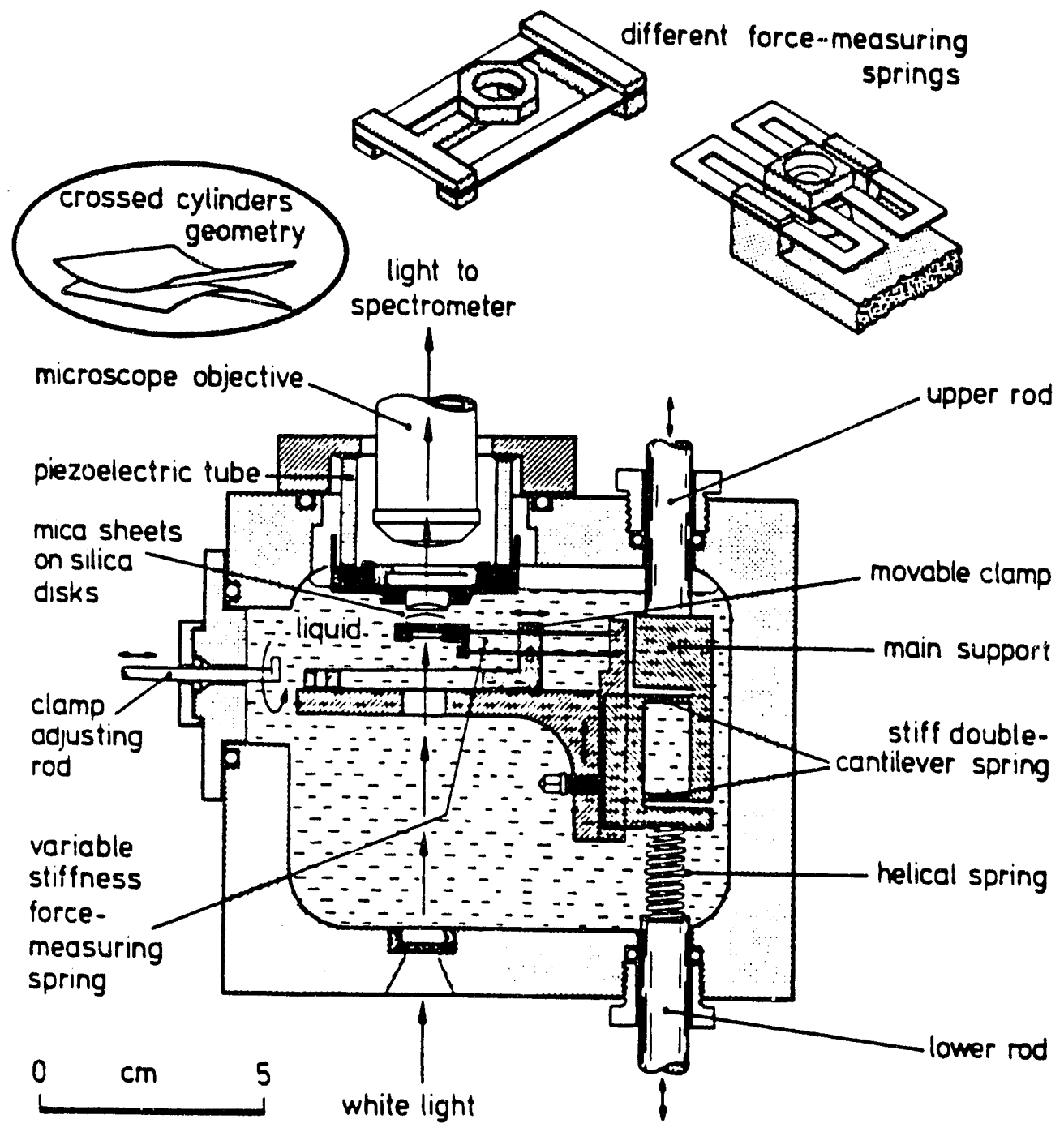

Figure 5. Schematic of the Surface Force Apparatus, Mark IV. 
two mica plates in distilled (Nanopure) water was determined. These results, which are shown in Figure 6, are in good agreement with curves reported in the literature (Herder, 1990), indicating that the apparatus was working correctly.

The next set of experimental surface force measurements were taken to determine the total interaction forces for a model hydrophobic system obtained by coating mica surfaces with dimethyldioctadecyl-ammonium (DDOA) bromide. This model system was used because the surface roughness and opacity of coal prevents it from being used directly in the Surface Force Apparatus. Recrystallized DDOA bromide was dissolved in a $95 / 5$ volume/volume mixture of hexane/ethanol and then spread on a pure water subphase in a Joyce Loebl Langmuir Mini-Trough housed in a laminar flow hood. This surfactant monolayer was then compressed at low speed and the surface pressure/head group area isotherm was measured to verify that the film was free of surface active impurities. This isotherm, which is shown in Figure 7, compared well with published isotherms, indicating that the monolayer was of acceptable purity. The spread monolayer was then transferred to silvered mica sheets already mounted on silica lens for use in the surface force apparatus. The deposition onto the mica sheets was performed at a surface pressure of $25 \mathrm{mN} / \mathrm{m}^{2}$. The coated mica surfaces emerged from the deposition dry and were immediately transferred to the surface force apparatus to avoid contamination by airborne dust. Contact angle measurements on identically prepared surfaces gave water contact angles in excess of $100^{\circ}$. Force-distance curves for the DDOA-coated surfaces were measured in distilled (Nanopure) water having a residual salt concentration of approximately $5 \times 10^{-6} \mathrm{M}$ monovalent ions, and in $\mathrm{KCl}$ solutions of $1 \times 10^{-3}, 1 \times 10^{-2}$ and $1 \times 10^{-1} \mathrm{M}$. As shown in Figure 8, the force-distance curve in pure water showed no repulsive double layer force, indicating that the coated surfaces were uncharged. This made the subsequent determination of the hydrophobic force much easier, since the Van 


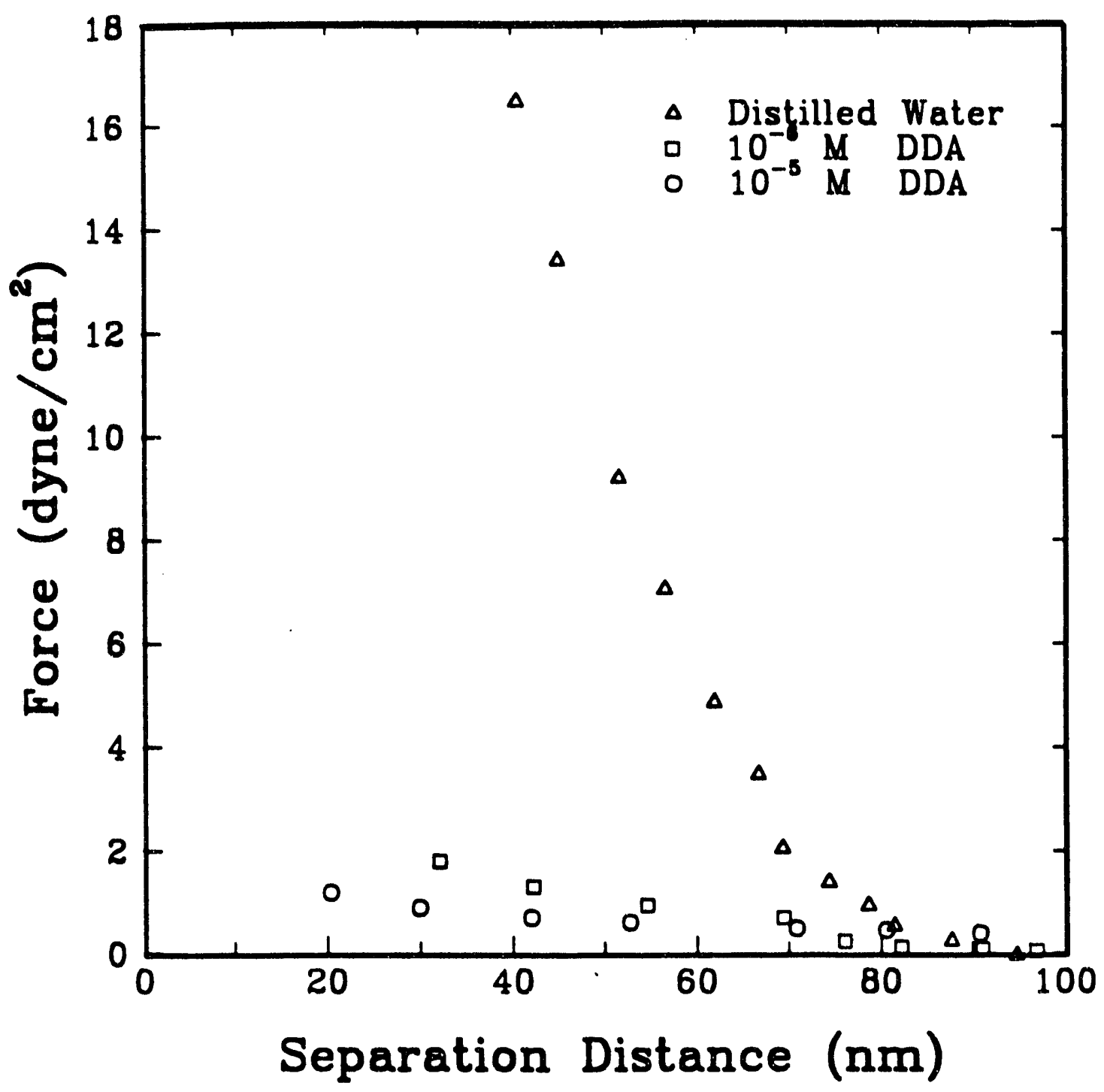

Figure 6. The effect of dodecylamine hydrochloride (DDA) addition on the surface interaction between two mica plates. 


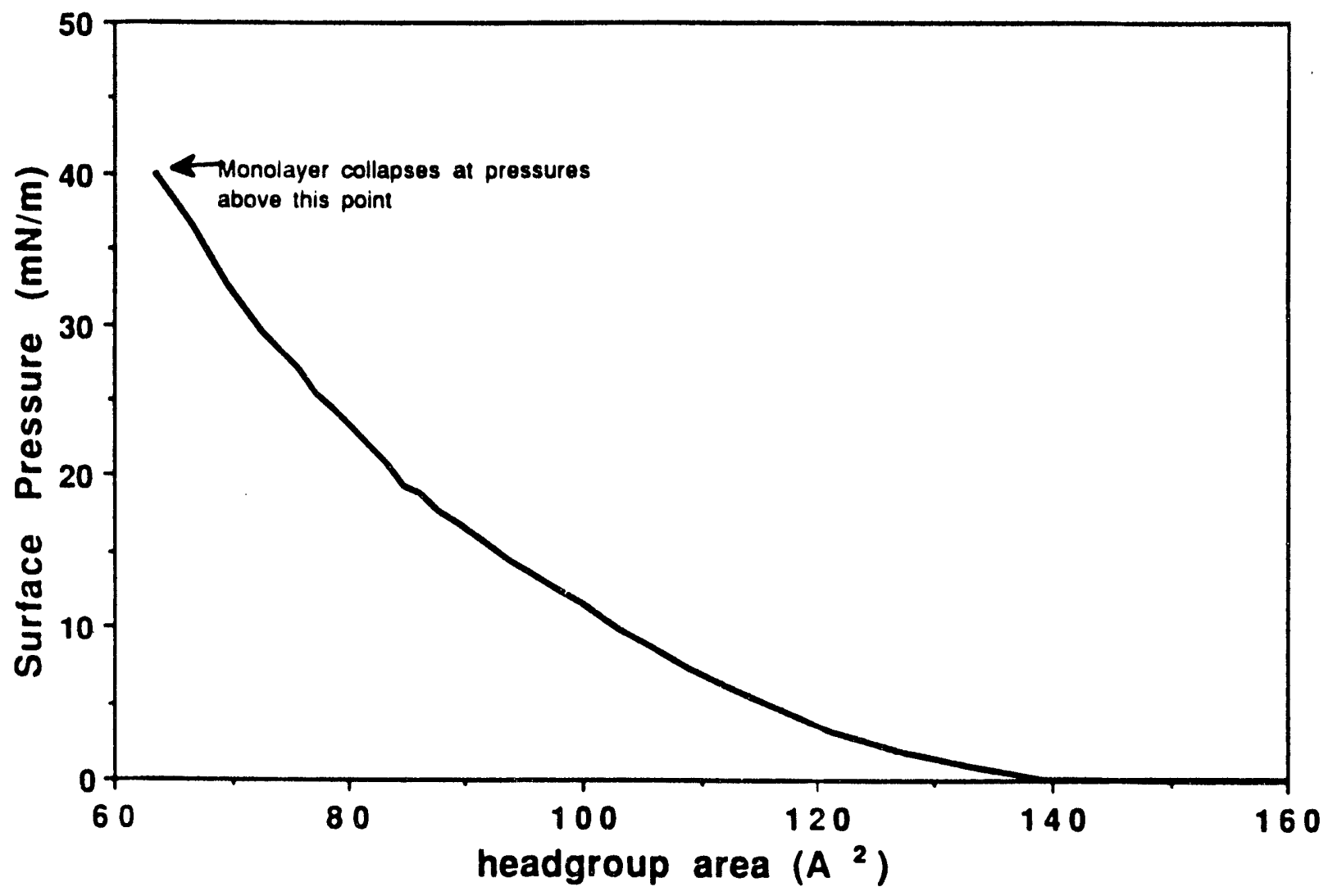

Figure 7. Surface pressure vs. head-group area isotherm for a monolayer of DDOA on pure water. 


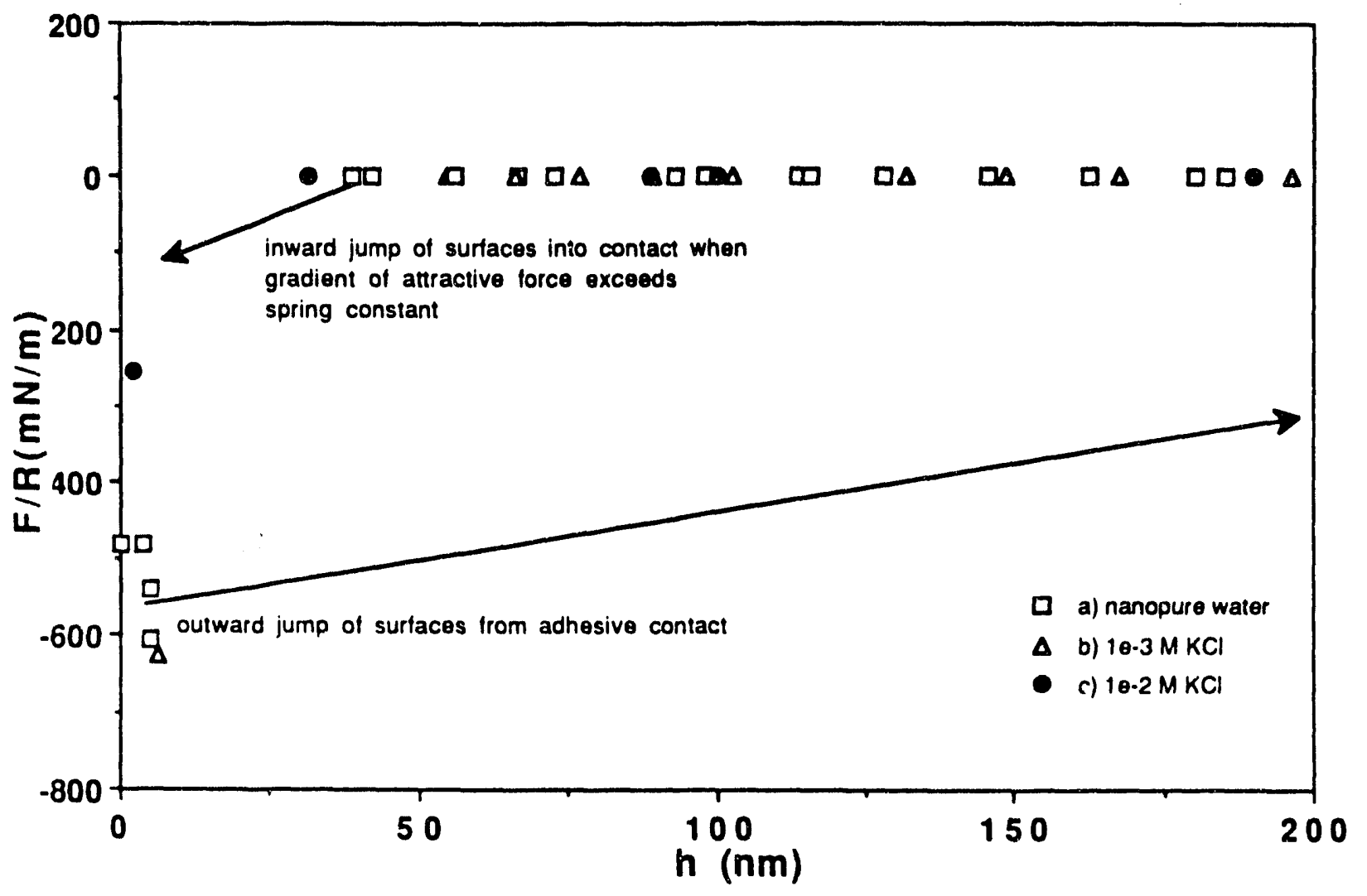

Figure 8. Force - distance curves for DDOA coated mica surfaces. 
der Waals attraction is the only other force to be subtracted. A small but measurable attractive force was observed at a surface separation of about $70 \mathrm{~nm}$, and the surfaces jumped into contact at a surface separation of about $40 \mathrm{~nm}$ (this is the point at which the gradient of the attractive force exceeds the spring constant of the leaf spring on which the lower surface is mounted). The adhesion force, i.e., the force required to separate the surfaces from contact, was found to vary from contact to contact within the range of 400 to $600 \mathrm{mN} / \mathrm{m}$. The value of the solid-liquid surface tension, $40.5 \mathrm{mN} / \mathrm{m}$, calculated from this estimate of the adhesion force, compared favorably with that obtained from Young's equation (Young, 1955) and the measured contact angle. Frequently, a strong repulsive force was found upon bringing the surfaces back into contact following separation of the surfaces from adhesive contact. This is attributed to surface damage due to the large force required to separate the surfaces. From the data, values of parameters $C$ and $D_{0}$ were found to be $2-4 \mathrm{mN} / \mathrm{m}$ and $11-16 \mathrm{~nm}$, respectively. These values are similar to previously reported values of these constants on extremely hydrophobic surfaces.

The addition of $1 \times 10^{-3} \mathrm{M} \mathrm{KCl}$ to the surface force apparatus had little effect on the force curve (Figure 8). Increasing the salt concentration to $1 \times 10^{-2} \mathrm{M} \mathrm{KCl}$ decreased the range of the attractive force and the depth of the adhesive well by about a factor of two (Figure 8). A further increase in the salt concentration to $1 \times 10^{-1} \mathrm{M} \mathrm{KBr}$ resulted in a repulsive force between the surfaces, which may result from the instability of the adsorbed monolayers. Although additional work beyond the scope of this project would be required to clarify this matter, it does appear that the range of the hydrophobic force is dependent upon salt concentration. The modeling equations may need to be modified to account for this, at least at high salt concentrations.

In an attempt to examine the hydrophobic interaction between oil-coated surfaces, mica surfaces that had been made hydrophobic using DDOA were coated with dodecane 
(a component of kerosene). Unfortunately, these preliminary experiments indicated that the adsorbed DDOA layer is not stable in this solvent (and therefore not stable in kerosene). Because of this, plans to test the effects of kerosene additions on the interaction between hydrophobic surfaces could not be completed as planned.

\section{b. Measurements of Bubble-Particle Detachment Force}

While the direct surface force measurements conducted using the Mark IV surface force apparatus can provide valuable information concerning particle-particle interactions, the measurements involve tedious procedures and are time-consuming. Since the objective of such measurements is to obtain information concerning hydrophobic interaction energy in general, it was deemed worthwhile to seek an easier experimental technique to obtain the same basic information. One such method involves the measurement of the interaction between a hydrophobic solid and an air bubble.

As shown in Figure 9, the maximum energy barrier against bubble-particle attachment can be calculated by subtracting the adhesion energy from the detachment energy. In the present work, the energy required to detach an air bubble from a methylated silica surface was measured using the procedure descrived by Janczuk (1983) and Barcicki et al. (1962). This technique is described in further detail in the following section. The adhesion energy was calculated from the equilibrium contact angles obtained using methylated silica plates. The net difference in detachment and adhesion energies represents the energy barrier against bubble-particle attachment, i.e., the activation energy for flotation.

The bubble-particle detachment studies were carried out using a methylated fused silica fiber with a flat-cut tip. The tip was immersed in the test solution in which a 2-mm diameter bubble was generated. After allowing five minutes to attain equilibrium, the 


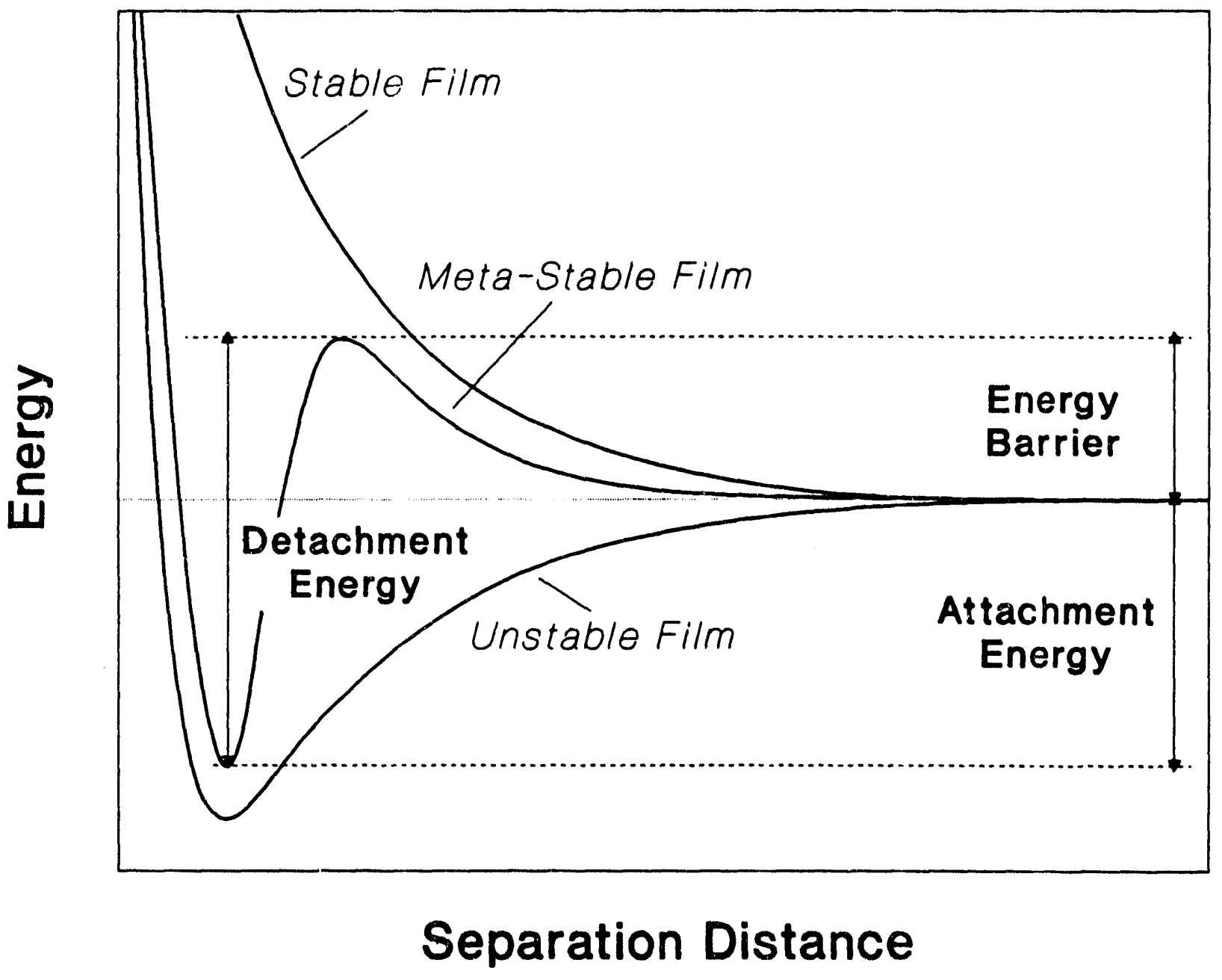

Figure 9. Schematic energy versus distance profiles for wetting films on solids. 
fiber tip was pressed against the air bubble until three-phase contact was achieved.

Detachment was initiated by slowly moving the air bubble away from the fiber tip with the help of a micrometer while monitoring the tip deflection with a cathetometer. The deflection of the tip was calibrated using a series of known weights. The detachment force was then calculated from the maximum deflection before detachment and divided by the surface area $\pi r_{k}^{2}$, where $r_{k}$ is the radius of the tip of the fiber, as measured by using an optical technique.

Equilibrium contact angles on the methylated silica plates were measured using a captive bubble technique. In these measurements, a plate and an air bubble of $1 \mathrm{~cm}$ diameter were left in the $\mathrm{KCl}$ solution for 5 minutes to ensure equilibrium between the surfaces and the solution prior to contact. Contact between the bubble and the plate was maintained for 5 minutes, after which the volume of the bubble was reduced until the contact perimeter jumped inward to ensure that the equilibrium was reached at advancing conditions. The contact angle was measured on both sides of the bubble after a 5-minute equilibration period. The reported contact angle values are the average over at least four repetitive measurements, with a reproducibility within $\pm 1^{\circ}$. From the measured values of contact angles, the work of adhesion of water on solid surfaces $\left(W_{N}\right)$ was calculated using a well-known relationship:

$$
W_{a}=\gamma_{v}(1+\cos \theta)
$$

The magnitude of $W_{1}$ can be used as a measure of the surface hydrophobicity. A small value for $\theta$ gives a large value of $W_{a}$, corresponding to a stronger interaction between water molecules and the solid surface, i.e. the surface is less hydrophobic.

In addition to the detachment and adhesion experiments, induction time ( $\tau)$ measurements were carried out using a procedure previously described by Yordan and 
Yoon (1988). In these measurements, one gram of methylated quartz particles was conditioned in $10^{-3} \mathrm{M} \mathrm{KCl}$ solution for 5 minutes and transferred to the glass cell for the induction time measurement. The cell with the sample was thermostabilized prior to measurement at $25 \pm 0.2^{\circ} \mathrm{C}$ using a temperature-controlled water bath. The time for $50 \%$ successful pick-up was recorded as the measured induction time.

Electrokinetic potential was measured with a Pen-Kem Model 501 Laser Zee Meter. The sample suspension was prepared by dispersing 0.02 grams of quartz particles $(-38 \mu \mathrm{m})$ in $500 \mathrm{ml}$ of $10^{-3} \mathrm{M} \mathrm{KCl}$ solution. After being mixed for 10 minutes, the suspension was allowed to settle for 3 minutes to avoid the coarse particles being carried into the electrophoresis cell. For a given experimental condition, at least three $\zeta$-potential readings from different runs were taken and averaged.

The force detaching an air bubble from a fused silica surface was measured as a function of solution $\mathrm{pH}$. The silica surface used in this experiment was methylated in $1 \mathrm{x}$ $10^{-3} \mathrm{M}$ TMCS solution and possessed a contact angle of $67^{\circ}$ in distilled water (pH 5-6). The measured values of the corresponding detachment force $\left(F_{d c}\right)$ are given in Figure 10. Also shown in this figure are the values of the adhesion force $\left(F_{a d}\right)$ calculated from the measured values of the contact angle $(\theta)$ and the radius of fused silica fiber $\left(r_{k}\right)$, using the following equation:

$$
F_{\alpha d}=-\frac{2 \gamma_{h}\left(\sqrt{2-\cos ^{2} \theta-\cos \theta}\right)}{2 r_{k}} .
$$

It is interesting to note that both $F_{\alpha d}$ and $F_{d e}$ decrease with increasing solution $\mathrm{pH}$ and that this decrease becomes more significant in alkaline solutions.

As shown in Figure 10, the force barrier $\left(F_{\max }\right)$ against bubble/particle adhesion, obtained from the difference between $F_{d c}$ and $F_{s d}$, increases with pH, i.e., from 6353 


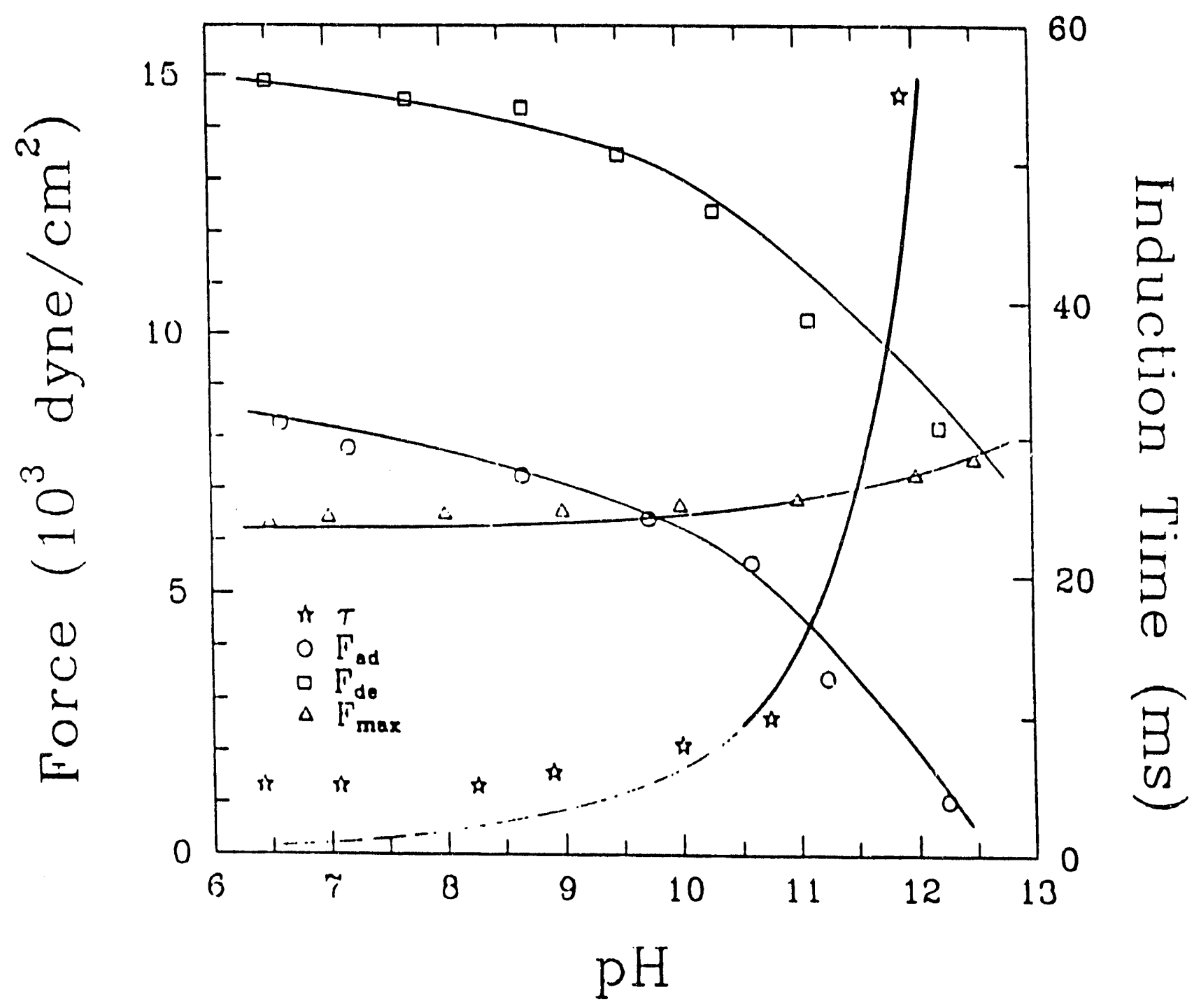

Figure 10. Variation of $\mathrm{F}_{\mathrm{de}}, \mathrm{F}_{\mathrm{dd}}, \mathrm{F}_{\max }$ and $r$ as a function of $\mathrm{pH}$, measured with silica surfaces methylated in $10^{-3} \mathrm{M}$ TMCS solution. 
dyne $/ \mathrm{cm}^{2}$ at $\mathrm{pH} 6.5$ to 7300 dyne $/ \mathrm{cm}^{2}$ at $\mathrm{pH} 12$. This is due to the fact that $F_{\text {ad }}$ decreases slightly faster than $F_{d c}$ as the $\mathrm{pH}$ increases. It is well known (Laskowski and Kitchener, 1969; Yoon and Yordan, 1986) that with increasing $\mathrm{pH}$, the surface potentials for both bubble and methylated silica surfaces increase, inducing an increased electrostatic doublelayer repulsion. As a result, the force barrier increases correspondingly. It is evident from Figure 10 that the induction time measured for quartz particles, methylated under the same conditions, also increases with increasing solution $\mathrm{pH}$, indicating that as the force barrier increases, the rate at which the disjoining film thins decreases. This suggests that the force barrier of bubble/particle interaction determines the thin film rupture and, hence, the adhesion (Laskowski and Iskra, 1970; Blake and Kitchener, 1972; Laskowski, Xu, Yoon, 1991).

In order to show the importance of various surface forces in bubble/particle adhesion, the magnitude of $F_{\max }, F_{d c}$ and $F_{\alpha}$ was also measured on less hydrophobic, fused silica surface methylated in $10^{-4} \mathrm{M}$ TMCS solution whose contact angle was $53^{\circ}$. The measurements were conducted by varying $\mathrm{KCl}$ concentration at constant $\mathrm{pH}$ of about 6.5 and are shown in Figure 11 . While $F_{\text {de }}$ is shown to decrease significantly with increasing $\mathrm{KCl}$ concentration above $10^{-2} \mathrm{M}$, changes in $F_{\star \Delta}$ are relatively small over the entire concentration range studied. As a result, the value of $F_{\max }$ decreases dramatically (see Figure 11). This accounts for an improved flotation response with increasing salt concentration (Schulze, 1984). A similar behavior was observed also by Read and Kitchener (1969) and Blake and Kitchener (1972) in connection with the wetting properties of thin films on silica and methylated silica surfaces. The impact of the salt concentration on decreased $F_{\text {max }}$ can be attributed to the screening effect of the supporting electrolyte (e.g., $\mathrm{KCl})$ upon the electrostatic double-layer force. 


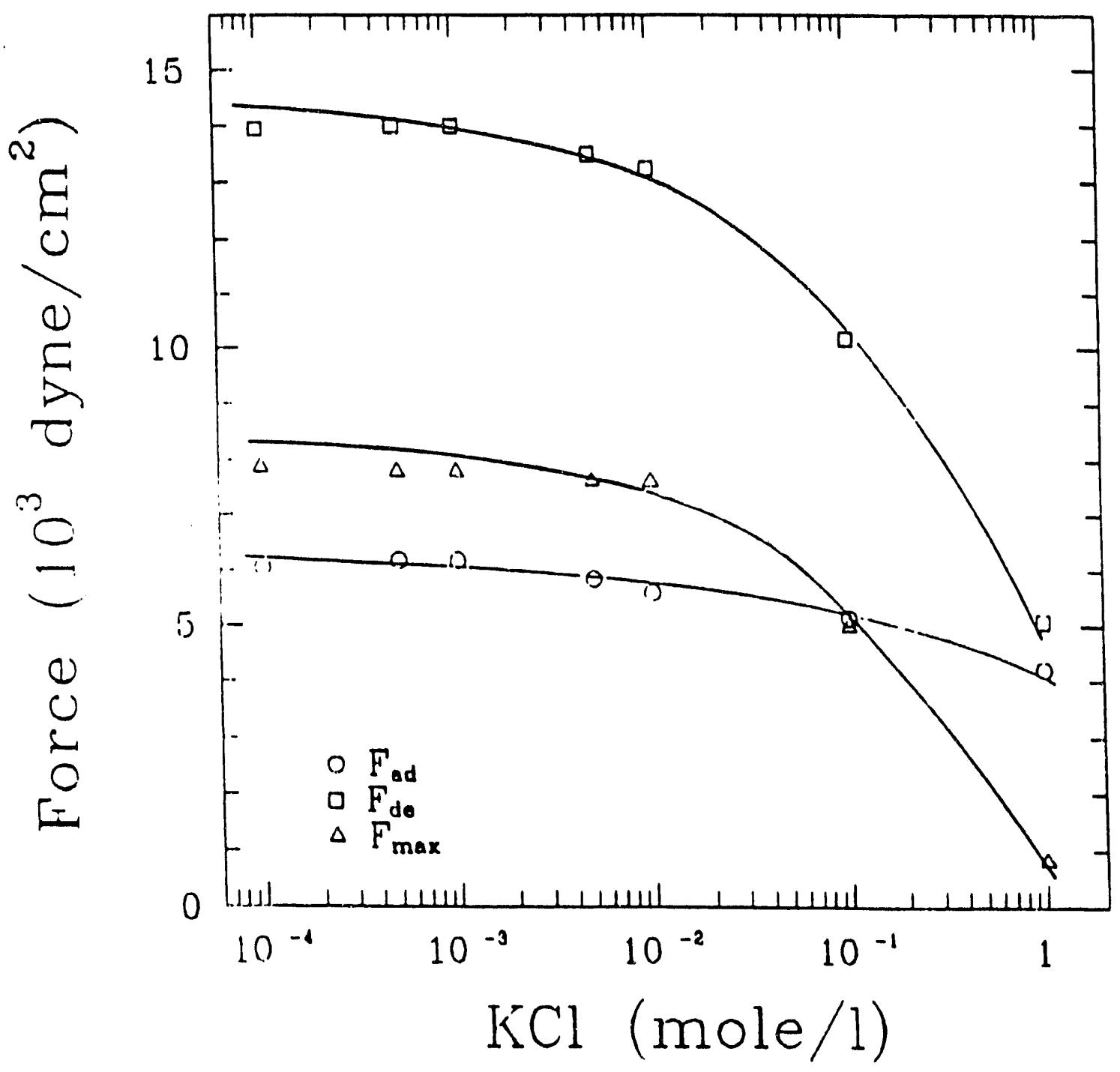

Figure 11. Variation of $\mathrm{F}_{\mathrm{de}}, \mathrm{F}_{\mathrm{sd}}$, and $\mathrm{F}_{\max }$ as a function of $\mathrm{KCl}$ concentration (pH about 6.5) measured with silica surfaces methylated in $10^{-4} \mathrm{M}$ TMCS solution. 
Figure 12 shows the experimental measurements of $F_{d e}$ and $F_{\Delta d}$ for surfaces having

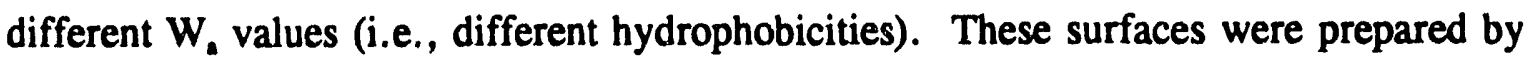
controlled methylation with varying concentrations of TMCS. The measurements were conducted at constant $\mathrm{pH}$ (about 6.5 ) and constant $\mathrm{KCl}$ concentration $\left(10^{-3} \mathrm{M}\right)$. As shown, both $F_{d e}$ and $F_{n d}$ increase with decreasing $W_{a}$, although $F_{a d}$ increases more rapidly. As a result, both $F_{\max }$ and the induction time decrease with decreasing $W_{a}$ (see Figure 12). These results confirm the previous conclusion, i.e., that the rate of thin film rupture and hence bubble/particle adhesion is dependent on the magnitude of the force barrier. For quartz particles with and without methylation, $\zeta$-potentials were also measured as a function of $\mathrm{pH}$. After methylation, the silica surface has a water contact angle of $70^{\circ}$ as measured with a fused silica plate methylated under the same conditions. The experimental results presented in Figure 13 indicate that $\zeta$-potential values are the same for both methylated and unmethylated quartz particles and their magnitudes increase with increasing solution pH. These findings are in agreement with those obtained in previous studies (Yoon, 1991; Laskowski and Kitchener, 1969; Blake and Ralston, 1985).

\section{c. Discussion}

In classical heterocoagulation theory, the total interaction force between an air bubble and a particle in a medium is described in terms of van der Waals dispersion force $\left(F_{\mathrm{D}}\right)$ and electrostatic double-layer force $\left(F_{\mathrm{E}}\right)$, i.e.,

$$
F_{T}=F_{D}+F_{E}
$$

The dispersion forces between an air bubble and a particle are generally repulsive (Kitchener, 1984; Laskowski, 1986; Dai et al., 1990; Schulze, 1984; Derjaguin, 1989; Usui et al., 1986). The magnitude of $F_{\mathrm{D}}$ can be calculated using the following approximation equation: 


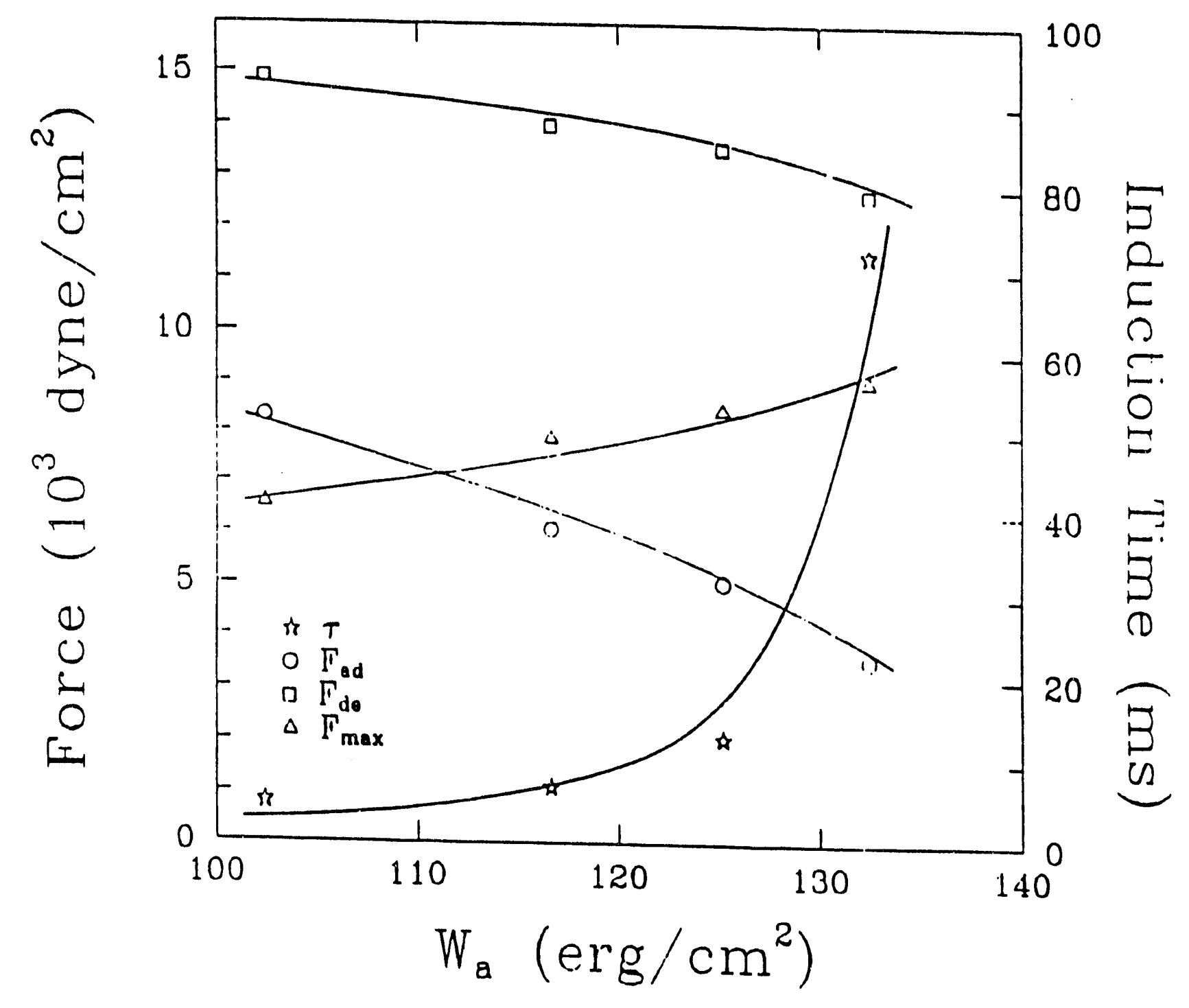

Figure 12. Dependence of $\mathrm{F}_{d e}, \mathrm{~F}_{\mathrm{ad}}, \mathrm{F}_{\mathrm{max}}$ and $\tau$ on $\mathrm{W}_{\mathrm{a}}$, obtained in $10^{-3} \mathrm{KCl}$ solution $(\mathrm{pH}$ about 6.5). 


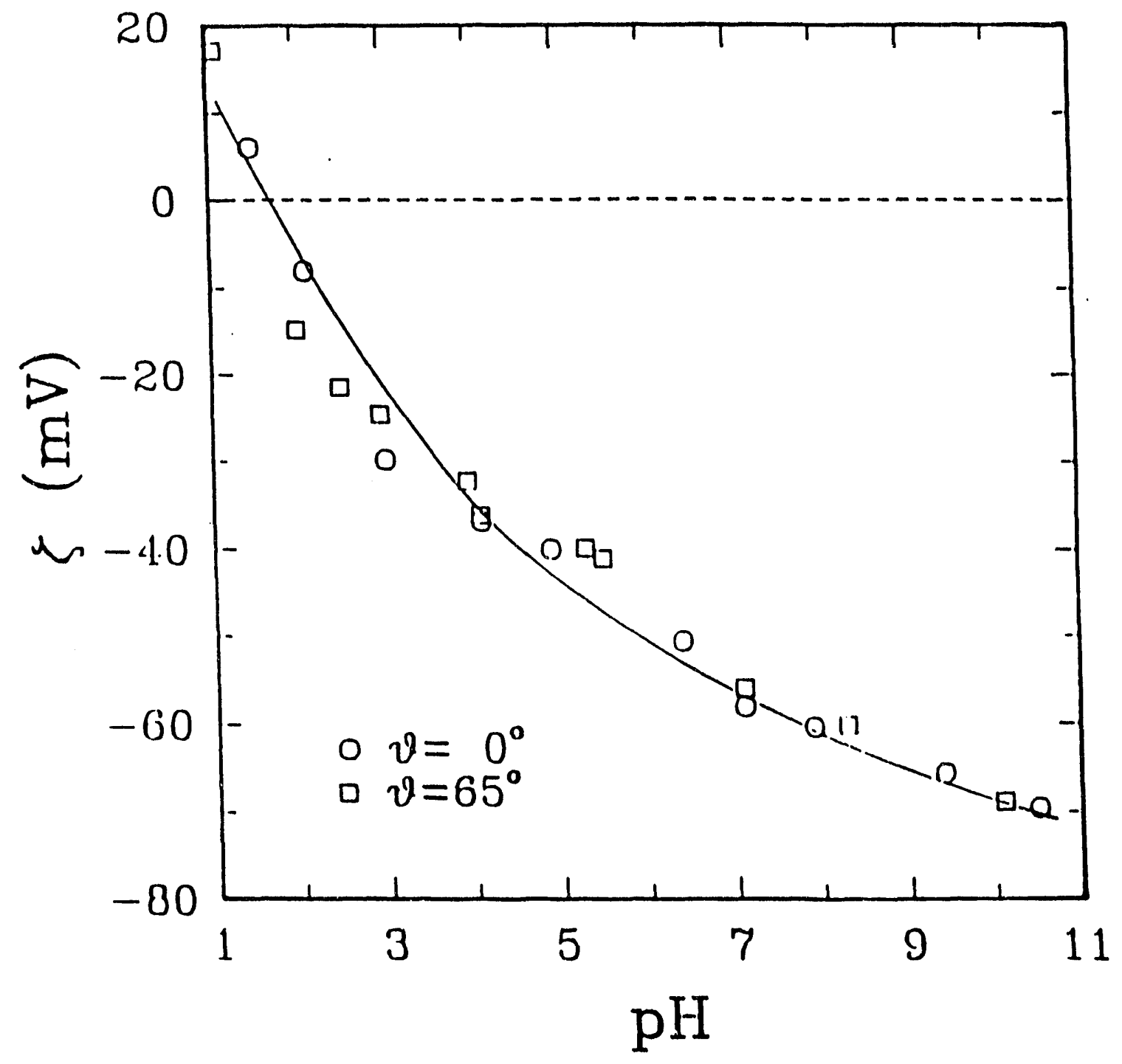

Figure 13. $\zeta$-potentials of silica particles with and without methylation in $10^{-3} \mathrm{M} \mathrm{KCl}$ solutions as a function of $\mathrm{pH}$. 


$$
F_{D}=-\frac{A_{S W B}}{6 \pi(H+28)^{3}}
$$

where $A_{\text {sWB }}$ is the Hamaker constant for silica/water/air system, $H$ is the distance separating Stern planes of silica and bubble surfaces, and $\delta$ is the Stern layer thickness. A value of $3.7 \AA$ was suggested by Devanathan and Tilak (1965) for $\delta$. Eq. [12] has been confirmed experimentally in a variety of systems (Blake, 1972). The electrostatic double-layer force can be calculated using the constant surface potential model equation (Blake, 1972'):

$$
F_{E}=-\frac{\varepsilon x^{2}}{8 \pi} \operatorname{cosech} \kappa H\left[\left(\Psi_{S}^{2}+\Psi_{B}^{2}\right) \operatorname{cosech} \kappa h-2 \Psi_{s} \Psi_{B} \operatorname{coth} \alpha H\right]
$$

where $\psi_{s}$ and $\psi_{B}$ are the surface potentials for silica and bubble, $\alpha$ is the Debye reciprocal length of the double-layer, and $\varepsilon$ is the dielectric constant of medium (water).

Using the expressions provided above, the interaction forces between an air bubble and silica surface can be calculated. The computations were carried out assuming a pH of 6.5 and an electrolyte $(\mathrm{KCl})$ concentration of $10^{-3} \mathrm{M}$. For this system, a Hamaker constant of $1.03 \times 10^{-13} \mathrm{erg}$ can be assumed, as suggested by Hough and White (1980). Also assumed were surface potentials of $-53 \mathrm{mV}$ and $-32 \mathrm{mV}$, approximated from the values of $\zeta$-potentials for silica and air bubble (Read and Kitchener, 1969; Yordan, 1989), and a calculated $\kappa$ value of $0.104 \mathrm{~nm}^{-1}$. The results of these calculations are plotted in Figure 14, where $F_{C}$ and $F_{E}$ are the dispersion and the electrostatic components, respectively, and $\mathrm{F}_{\mathrm{T}}$ is total interaction force.

Figure 14 shows that the force profile has a maximum which is conventionally referred to as the force barrier. It is the magnitude of this force barrier that determines

'There is a printing error in Eq. (6) in this reference. 


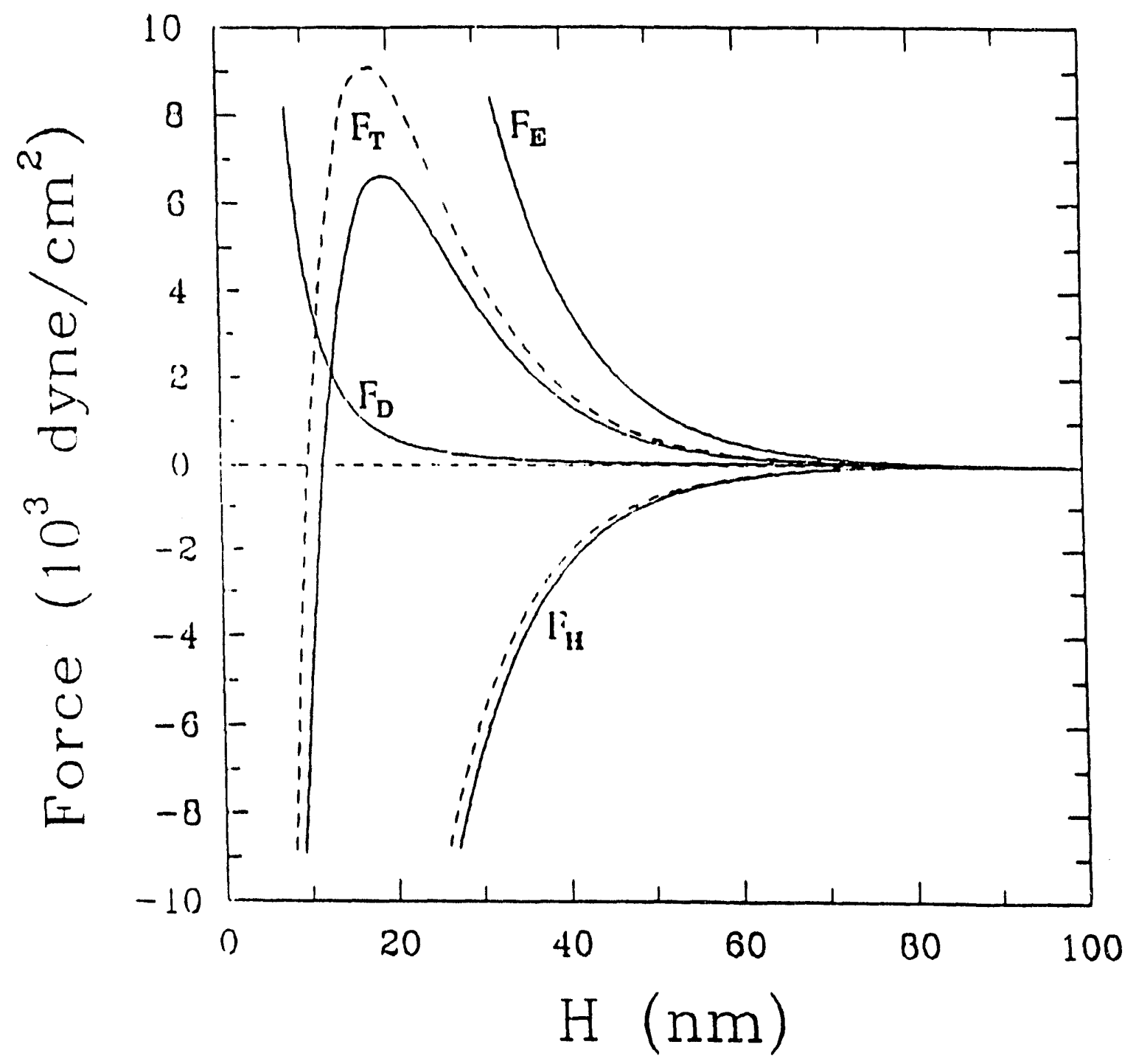

Figure 14. Surface force profile calculated with Eq.[12] for an air bubble and a silica particle in $10^{-3} \mathrm{M} \mathrm{KCl}$ solution ( $\mathrm{pH} 6.5$ ). 
the stability of a thin film between an air bubble and a solid surface. The importance of this parameter led us to investigate the force barriers for other $\mathrm{pH}$ values. The calculated results $\left(\mathrm{F}_{\max }{ }^{\mathrm{C}}\right)$ are summarized in Table I. Also included in this table are the $\zeta$-potential values for air bubble $\left(\zeta_{\mathrm{B}}\right)$ and silica $\left(\zeta_{\mathrm{S}}\right)$ surfaces and the corresponding experimental values for force barriers $\left(F_{\max }{ }^{M}\right)$.

Table I. Comparison of force barriers calculated using the conventional heterocoagule: on theory and experimental values determined as a function of $\mathrm{pH}$

\begin{tabular}{|c|r|r|r|r|}
\hline $\mathrm{pH}$ & 6.5 & 7.5 & 8.5 & 9.5 \\
\hline \hline$\zeta_{\mathrm{B}}(\mathrm{mV})^{*}$ & -32.0 & -41.0 & -50.0 & -58.0 \\
\hline$\zeta_{\mathrm{S}}(\mathrm{mV})$ & -53.0 & -58.7 & -63.0 & -70.0 \\
\hline $\mathrm{F}_{\max }{ }^{\mathrm{c}}\left(\right.$ dyne $\left./ \mathrm{cm}^{2}\right)$ & 43270.3 & 88131.7 & 768192.9 & 1094887.0 \\
\hline $\mathrm{F}_{\max }{ }^{\mathrm{M}}\left(\right.$ dyne $\left./ \mathrm{cm}^{2}\right)$ & 6353.0 & 6500.0 & 6558.8 & 6648.1 \\
\hline
\end{tabular}

*Ruckenstein and Churaev (1991)

As seen from Table I, the magnitude of $\zeta$-potential increases with increasing solution $\mathrm{pH}$ for both the air bubble and methylated silica surfaces. Accordingly, the electrostatic double-layer repulsion and, hence, the force barrier (calculated using the classical DLVO theory) increase (Table I). This observation is in general agreement with the experimental results. However, the measured force barrier is at least ten times smaller than the calculated value, a discrepancy which suggests that an attractive hydrophobic force must be included in the classical DLVO theory in order to extend its application.

It is important to mention that the classical DLVO theory cannot account for the observed decrease of the force barrier with increasing surface hydrophobicity (Figure 12). 
Since the experiments were conducted in solutions of the same $\mathrm{pH}$, all the bubbles have the same $\zeta$-potential value. Figure 13 shows that at a given $\mathrm{pH}$, the $\zeta$-potential of methylated hydrophobic silica and pure hydrophilic silica are practically identical. According to the classical DLVO theory, the electrostatic double-layer force between air bubbles and silica surfaces should then remain unchanged. On the other hand, the van der Waals dispersion force may be slightly reduced due to the formation of a hydrophobic surface layer. However, this change may be negligible over a large separation distance (Vincent, 1973), which implies that the force barriers calculated using the DLVO theory should be identical regardless of the surface hydrophobicity. However, this contradicts our experimental results. Therefore, in order to account for the observed reduction of the force barrier, an attractive hydrophobic force $\left(F_{\mathrm{H}}\right)$, which increases with increasing surface hydrophobicity, must be included in the conventional heterocoagulation theory. This "extended" DLVO theory is given by:

$$
F_{T}=F_{D}+F_{E}+F_{B},
$$

where $F_{\mathrm{H}}$ is the hydrophobic interaction force. $\quad F_{\mathrm{H}}$ can be calculated using Israelachvili's exponential force law (Israelachvili and Pashley, 1982), i.e.:

$$
F_{H}=-C \exp \left(-\frac{H}{D_{0}}\right)
$$

in which $D_{0}$ is the decay length and $C$ is the force constant. With Eq. [15], the hydrophobic interaction force can be quantified provided that $D_{0}$ and $C$ are known. From the data available in this work, it is difficult to determine both parameters simultaneously. However, with strongly hydrophobic solid surfaces, it is likely that the decay length $\left(D_{0}\right)$ is in the 10-15 nm range (Yoon, 1991; Ruckenstein and Churaev, 1991). It is also recognized that the strength of the hydrophobic force is regulated by the surface 
hydrophobicity, while the decay length is mostly related to the nature of the medium (Dai et al., 1990). By assuming a decay length of $10 \mathrm{~nm}$ as an example, the force constant was determined in such a way that the measured force barrier would match the value calculated using Eq. [14]. A $C$ value of $1.332 \times 10^{5} \mathrm{mN} / \mathrm{m}$ was obtained for the interaction between a silica surface methylated with $10^{-3} \mathrm{M}$ TMCS solution and an air bubble in $10^{-3} \mathrm{M} \mathrm{KCl}$ solution of $\mathrm{pH} 6.5$. This value is one order of magnitude greater than that obtained for the interactions between two strong hydrophobic silica surfaces (Israelachvili and Pashley, 1984). This suggests that the hydrophobic force between an air bubble and a hydrophobic surface is stronger than that between two hydrophobic solid surfaces. Experimentally, it has also been observed (Blake and Kitchener, 1972; Yordan and Yoon, 1991) that the thin films between air bubble and hydrophobic solid surfaces rupture at a much thicker film thickness, indicating the existence of a stronger long-range hydrophobic force.

The hydrophobie force constants for the other solid surfaces of different hydrophobicities were also calculated using decay lengths of 10 and $12 \mathrm{~nm}$, and the corresponding force constants $C_{\mathrm{I}}$ and $C_{\mathrm{I}}$, respectively, are presented in Table II. This data shows that the hydrophobic force constant increases with increasing surface hydrophobicity. It is also interesting to note that changing the decay length from $10 \mathrm{~nm}$ to $12 \mathrm{~nm}$ in the above calculation does not significantly affect the outcome of the calculated force constants. 
Table II. The values of the hydrophobic force constant calculated from the measured force barrier as a function of surface hydrophobicity.

\begin{tabular}{|c|r|r|r|r|}
\hline$W_{1}$ & 132.4 & 125.2 & 116.6 & 102.4 \\
\hline \hline$F_{\max }{ }^{M}\left(\right.$ dyne $\left./ \mathrm{cm}^{2}\right)$ & 9092.5 & 8489.5 & 7909.7 & 6589.6 \\
\hline$C_{1}\left(10^{3}\right.$ dyne $\left./ \mathrm{cm}^{2}\right)$ & -118.8 & -122.1 & -123.3 & -128.3 \\
\hline$C_{\text {II }}\left(10^{3}\right.$ dyne $\left./ \mathrm{cm}^{2}\right)$ & -117.7 & -121.4 & -124.9 & -133.2 \\
\hline
\end{tabular}

As another example, the hydrophobic force $\left(\mathrm{F}_{\boldsymbol{H}}\right)$ operating between air bubbles and silica surfaces methylated in $10^{-6}$ and $10^{-3} \mathrm{M}$ TMCS solutions have been calculated using Eq. [15]. The results of these calculations are given in Figure 15, along with the van der Waals and electrostatic double-layer forces present in the system. As shown, the hydrophobic interaction force is primarily responsible for reducing the force barrier against bubble-particle attachment and is the major driving force for overcoming the longrange van der Waals and electrostatic double-layer repulsive forces. Since bubbles are assumed to have the same surface hydrophobicity (provided that the solution conditions are the same as in the present case), the observed increase in the hydrophobic force with reagent concentration should be attributed to the enhanced hydrophobicity of the silica surface. It is also worth mentioning that the origin of the hydrophobic force is still uncertain, although its existence and long-range nature have been demonstrated for many other systems. Possible mechanisms for long-range hydrophobic forces have been postulated and summarized in a recent note by Ruckenstein and Churaev (1991).

As shown previously in Figure 11, the force barrier decreases with increasing electroiyte concentrations. This finding can be explained by the decrease in the $\zeta$ potentials of the bubble and particle due to double-layer compression. As the repulsive electrostatic force decreases, so does the force barrier. On the other hand, it is also possible that an increase in salt concentration could decrease contributions from the 


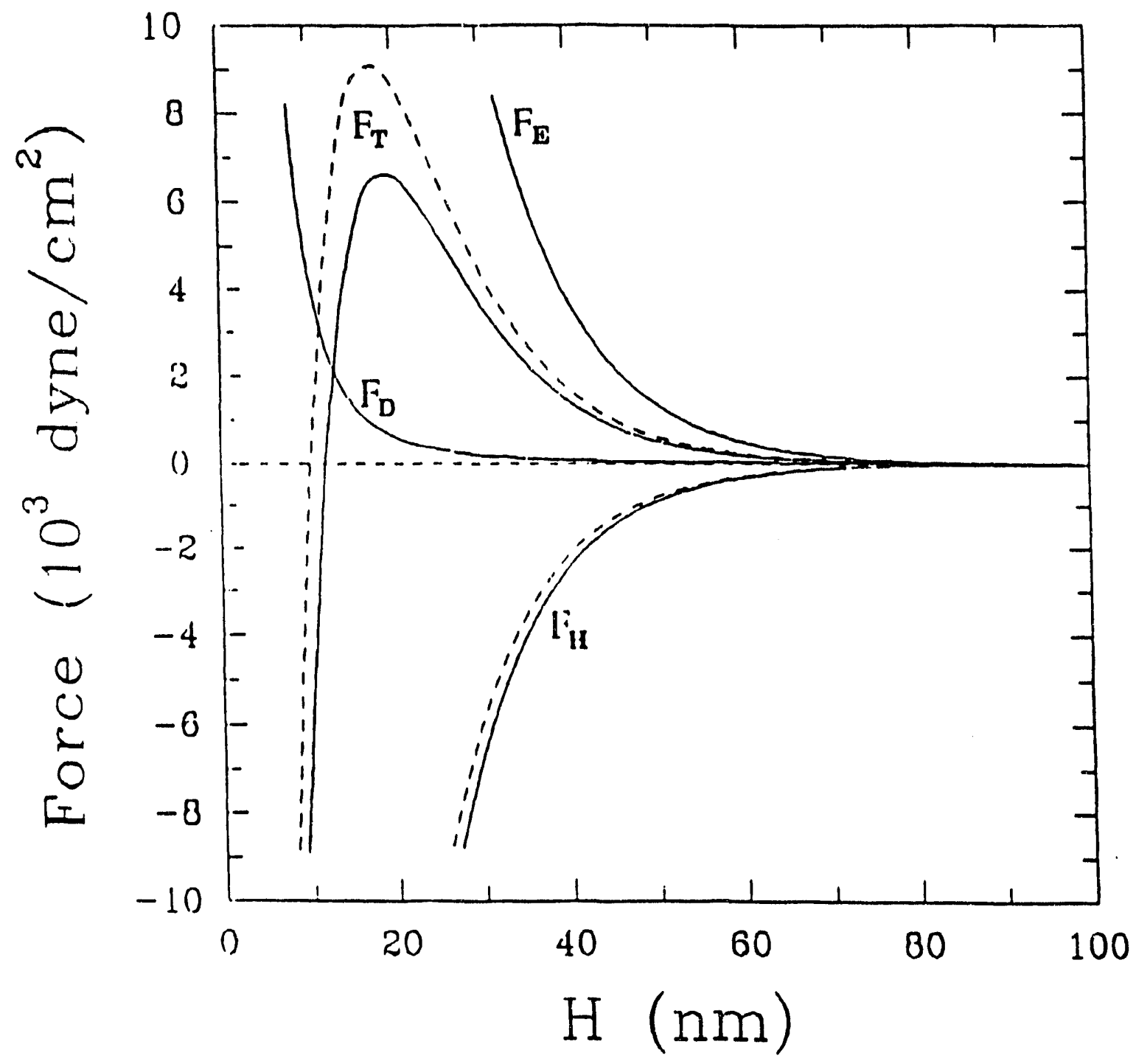

Figure 15. Surface force profile calculated for silica particles methylated in $10^{-6} \mathrm{M}$ (dashed) \& $10^{-3} \mathrm{M}$ (solid) TMCS solutions, interacting with air bubbles in $10^{-3}$ $\mathrm{M} \mathrm{KCl}$ solution (pH 6.5). 
hydrophobic force to the force barrier. It has been experimentally observed that the longrange hydrophobic forces between two hydrophobic solid surfaces decrease with increasing salt concentrations (Christensen et al., 1989).

With the exception of magnitude of the forces involved, both the particle-particle and bubble-particle systems have been treated in essentially the same manner in the present work. However, one key difference does exist that has not been addressed. While the force of particle-bubble adhesion (Eq. [10]) is calculated from the "free" contact angle measurements using flat polished plates, the detachment force is measured on a small rod of a given size. The hydrophobicity of these two surfaces are probably identical, but it is quite likely that the contact angle in the moment of detachment is different from the "free" contact determined on a large plate. This is caused by a deformation of the bubble in the moment of attachment or detachment. This phenomenon does not occur during particle-particle interactions, but it may have a significant impact on the force required to detach the particle from the bubble surface. As a result, the theoretical values calculated in the present work may differ slightly from those that actually occur during the act of flotation.

\section{Task 2.2 Determination of Hydrophobic Interaction Parameters}

Samples for the determination of the hydrophobic interaction parameters for fresh and oxidized Elkhorn No. 3 and Pittsburgh No. 8 coals were obtained by performing heavy media separations on $+1 / 4$-inch ROM coal at a specific gravity of 1.3 . This procedure gave clean coal products containing approximately $4 \%$ ash (for the Elkhorn No. 3 sample) and $3 \%$ ash (for the Pittsburgh No. 8 coal). Several chunks of the precleaned coal were cut and polished for the contact angle measurements. The 
remaining coal was crushed and screened at 400 mesh. The -400 mesh material was collected for the batch coagulation tests and the zeta potential $(\zeta)$ measurements. A fraction of the precleaned Elkhorn No. 3 coal was also heat treated in a laboratory furnace for 24 hours at $140^{\circ} \mathrm{C}$ to study the effect of oxidation.

The batch coagulation tests to establish the effective operating range for these two samples were conducted over a $\mathrm{pH}$ range of $2-11$. In these tests, a 10 -gram coal sample was suspended in a $500-\mathrm{ml} \mathrm{KCl}$ solution $\left(10^{-3} \mathrm{M}\right)$ and agitated for 5 minutes at $750 \mathrm{rpm}$. After agitation, the suspension was allowed to settle for 3 minutes and then $310 \mathrm{ml}$ of the supernatant was removed via siphoning. The solids in the supernatant were u eighed after filtration and drying. Coagulation efficiency $\left(E_{\mathrm{c}}\right)$ was calculated as:

$$
E_{c}=100\left(W_{i}-W_{j}\right) / W_{i} \text {, }
$$

in which $W_{i}$ is the initial weight of solids in a given volume of suspension before coagulation and $W_{f}$ refers to the weight of solids remaining in the supernatant after coagulation.

Coagulation efficiency values for fresh and oxidized Elkhorn No. 3 coals are shown as a function of slurry $\mathrm{pH}$ in Figure 16. For the fresh coal samples, high coagulation efficiency values were obtained throughout the acidic $\mathrm{pH}$ range and up to a $\mathrm{pH}$ of 8.2. $\mathrm{A}$ further increase in $\mathrm{pH}$ resulted in a sharp decrease in coagulation efficiency. The coagulation efficiency values for the oxidized coal samples remained high between the pH values of 3 and 5.4. However, the efficiency values declined below a $\mathrm{pH}$ of 3 and above a $\mathrm{pH}$ of 5.4. Thus, the upper critical coagulation $\mathrm{pH}(\mathrm{pH})$ for the fresh and oxidized Elkhom No. 3 coal samples was found to be 8.2 and 5.4, respectively. High coagulation efficiencies were also obtained for the Pittsburgh No. 8 coal between the $\mathrm{pH}$ values of 2.5 and 6.2 (Figure 17). However, an increase in $\mathrm{pH}$ beyond 


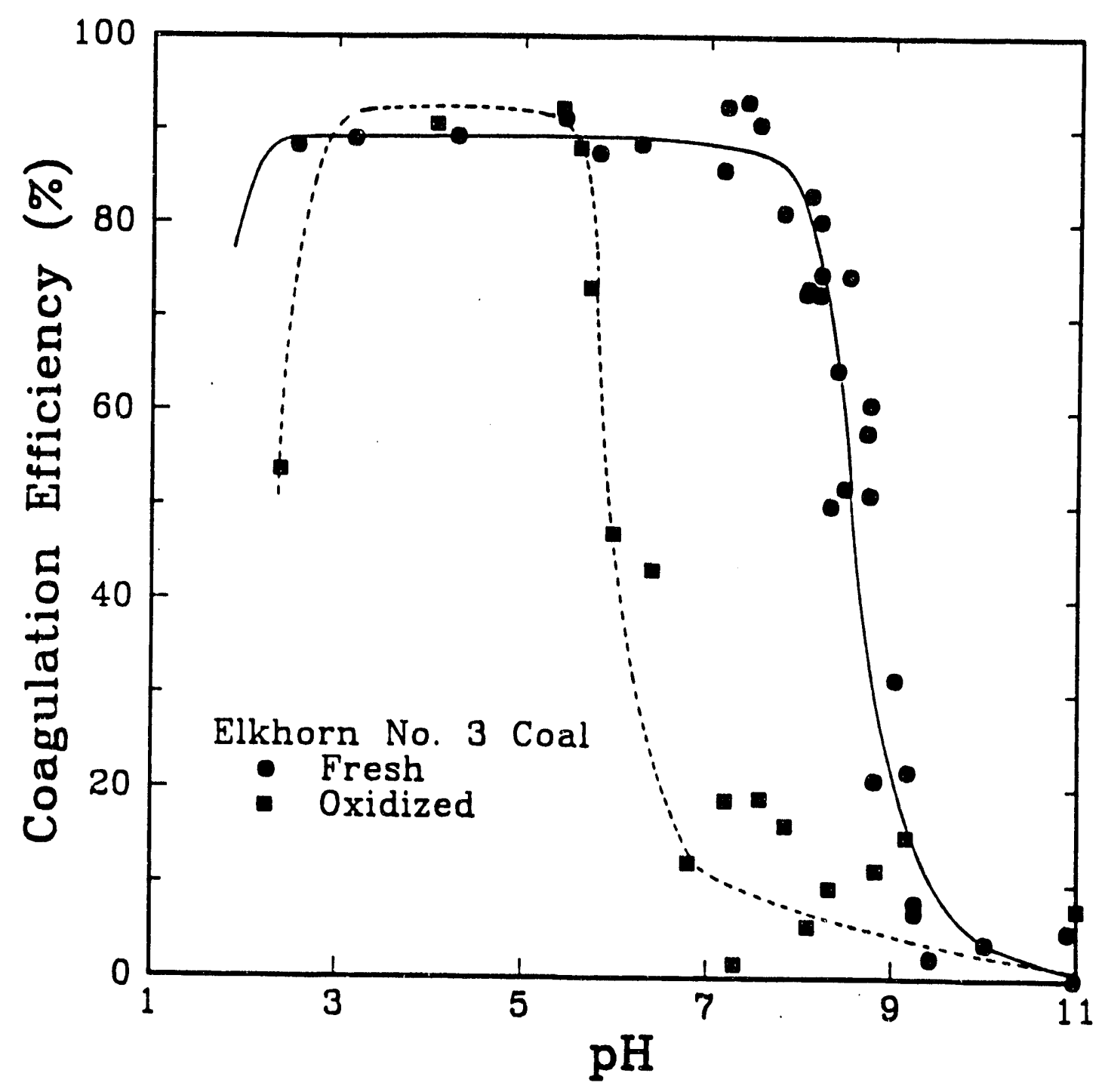

Figure 16. Effect of solution pH on the coagulation efficiency of fresh and oxidized Elkhorn No. 3 coal. 


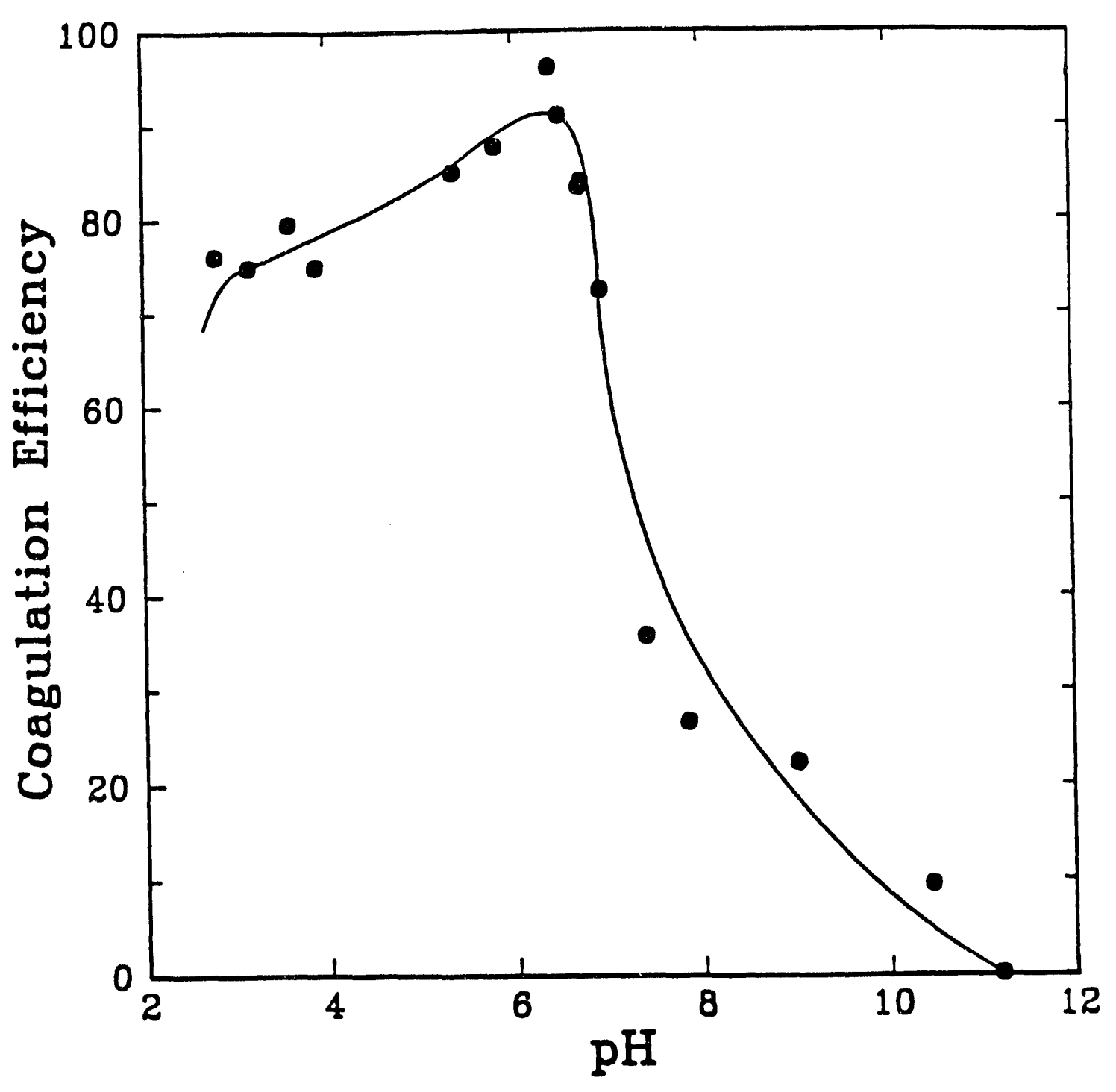

Figure 17. Effect of solution $\mathrm{pH}$ on the Coagulation Efficiency (as defined by Eq.[2]) of Pittsburgh No. 8 coal. 
6.2 resulted in a sharp decrease in coagulation efficiency (i.e., a stable suspension was formed). Based on this data, it was concluded that the upper critical coagulation pH limit, $\mathrm{pH}_{\mathrm{c}}$, was 6.2 for this particular sample of coal.

The extended DLVO theory, i.e., Eqs. [4]-[16], has been used to assess the behavior of these two systems. The procedures used and results obtained for the estimation of the basic parameters required to calculate the hydrophobic interaction energy, $\left(V_{H}\right)$, the dispersion interaction energy, $\left(V_{A}\right)$, and the electrostatic repulsive energy $\left(V_{R}\right)$ are discussed below.

a) The water and methylene iodide contact angles required to determine the magnitude of the hydrophobic interaction energy term, $\left(V_{H}\right)$, were measured at $25^{\circ} \mathrm{C}$ using a Ramé-Hart contact angle goniometer. The sessile drop technique was employed in a closed container to take the measurements at equilibrium vapor pressure.

b) The Hamaker constant, $A_{131}$, required to determine the dispersion interaction energy, $\left(V_{A}\right)$, was estimated using the following expression:

$$
A_{131}=\frac{\left[\sqrt{A_{11}}-\sqrt{A_{33}}\right]^{2}}{1-2.5 \times 10^{18} \sqrt{A_{11} A_{33}}},
$$

in which $A_{11}$ and $A_{33}$ refer to the Hamaker constants of the solid and medium, respectively, in vacuum. Fowkes (1964) showed that these two constants can be determined from the equation:

$$
A_{u}=6 \pi r_{i i}^{2} \gamma^{d}
$$

where $r_{\mathrm{ii}}$ is the intermolecular distance within the interacting body of the substance and $\gamma^{d}$ refers to the dispersion component of the surface free energy of the 
substance in question. Fowkes suggested that $6 \pi r_{\mathrm{ii}}$ approximates $1.44 \times 10^{-14}$ for most materials.

c) Zeta potential ( () measurements are commonly used as an estimate of the Stern potential term in the electrostatic repulsive interaction energy $\left(V_{R}\right)$ equation. Results over a pH range of 2 to 11 were obtained with a Pen Kem Model 501 Lazer-Zee meter. The sample suspensions used for these measurements were prepared by dispersing 0.02 grams of the -400 mesh coal samples in $500 \mathrm{ml}$ of $10^{-3} \mathrm{M} \mathrm{KCl}$ solution.

For the Elkhorn No. 3 coal, the methylene iodide contact angles $\left(\theta_{m}\right)$ for the fresh and oxidized coal samples were measured to be $20^{\circ}$ and $15^{\circ}$, respectively. From these values, the dispersion component of the surface free energy $\left(\gamma^{d}\right)$ of the fresh coal sample was calculated to be $50.05 \mathrm{~mJ} / \mathrm{m}^{2}$. The same constant for the oxidized coal was determined to be $51.41 \mathrm{~mJ} / \mathrm{m}^{2}$. In order to determine the values of $W_{a}^{\text {nd }}$ for these two samples, water contact angles $\left(\theta_{w}\right)$ were measured and used along with the $\gamma_{s}^{d}$ values discussed earlier. For the fresh Elkhorn No. 3 coal, the water contact angle was found to be $67^{\circ}$, which resulted in a $W_{a}^{n d}$ value of $33.68 \mathrm{~mJ} / \mathrm{m}^{2}$. The $\theta_{w}$ and $W_{a}^{\text {md }}$ values for the oxidized coal were determined to be $63^{\circ}$ and $37.38 \mathrm{~mJ} / \mathrm{m}^{2}$, respectively. The increase in the $W_{a}^{\text {nd }}$ value reflects the decrease in hydrophobicity for the oxidized coal sample. The hydrophobic constants, $C$, were calculated to be $-1.198 \mathrm{~mJ} / \mathrm{m}^{2}$ for the fresh coal and $0.416 \mathrm{~mJ} / \mathrm{m}^{2}$ for the oxidized coal by using Eq. [12]. Using the $\gamma_{\triangleleft}$ values listed above, the Hamaker constant for the fresh sample of Elkhorn No. 3 coal was calculated to be $7.207 \times 10^{-20} \mathrm{~J}$. For the oxidized sample, the Hamaker constant was $7.403 \times 10^{20} \mathrm{~J}$. The zeta-potentials $(\zeta)$ used for determining $V_{R}$ for the Elkhorn No. 3 samples are shown plotted versus $\mathrm{pH}$ in Figure 18. The isoelectric points (i.e.p.) of the fresh and oxidized coal samples were found to be 4.2 and 3.5 , respectively. The zeta-potential 


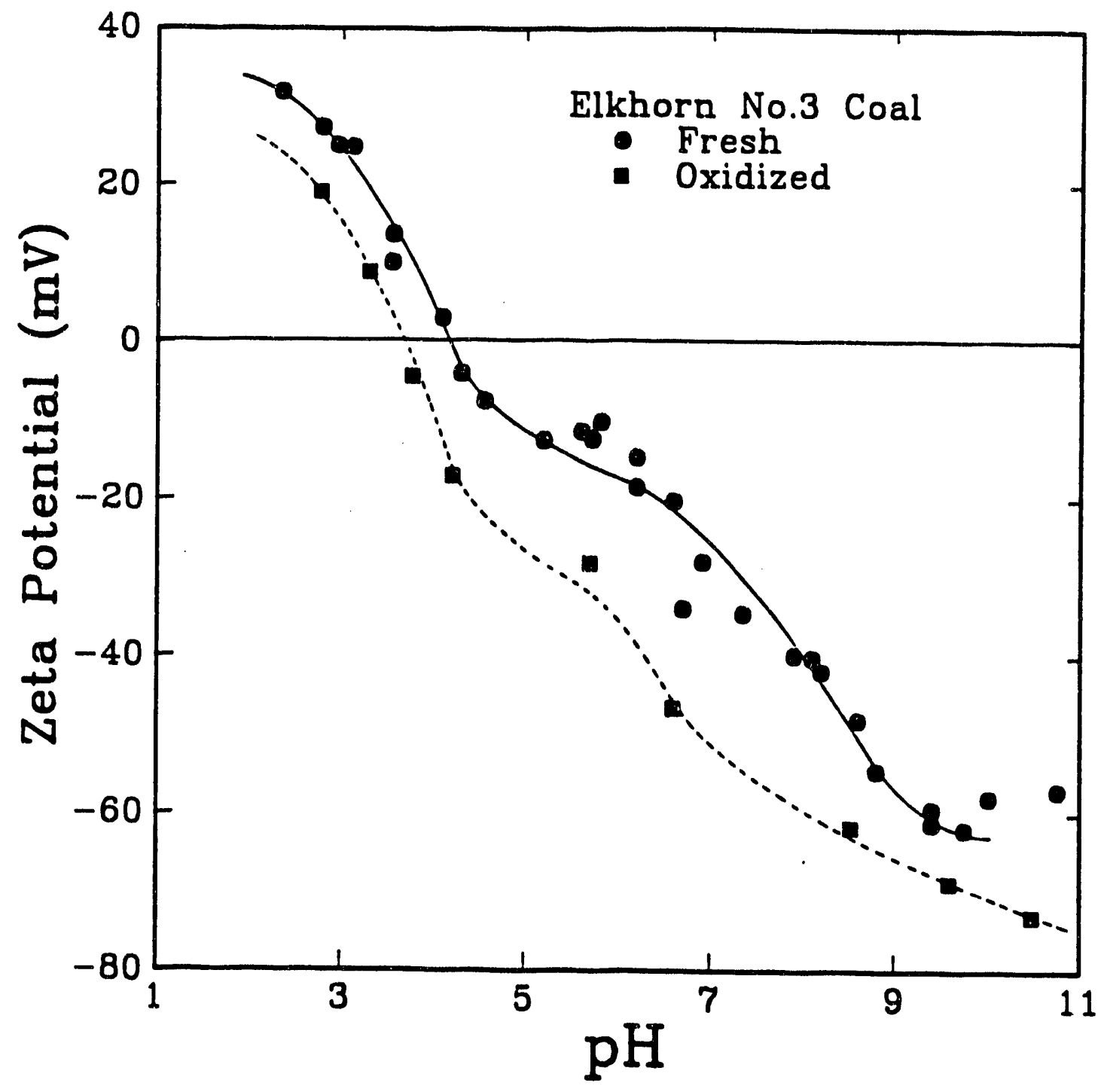

Figure 18. Zeta potential $(\zeta)$ measured for fresh and oxidized Elkhorn No. 3 coal particles as a function of $\mathrm{pH}$. 
corresponding to the $p H_{c}$ for the fresh coal was $-43 \mathrm{mV}$. As expected, this value decreased after oxidization to $-26 \mathrm{mV}$.

The potential energy vs. distance diagram showing the contributions of the various surface forces toward the total interaction energy $\left(V_{T}\right)$ at the critical coagulation zeta potential $\left(\zeta_{c}\right)$ of the fresh Elkhorn No. 3 coal is shown plotted as a function of the separation distance in Figure 19. The maximum total interaction energy $\left(V_{T, \max }\right)$ or energy barrier was $36 k \mathrm{~T}$, and was dominated by the electrostatic and hydrophobic interaction energies. Coagulation at such a small energy barrier can be induced by gentle mechanical agitation. By using the zeta potential $(\zeta)$ values obtained over a pH range of 2.5-11, $V_{T, \text { max }}$ was calculated as a function of $\mathrm{pH}$ for both the fresh and oxidized coal samples as shown in Figures 20 and 21. The figures compare the calculated energy barriers with the experimental coagulation efficiency values. In both cases, an increase in the calculated energy barrier corresponded with the critical coagulation $\mathrm{pH}\left(\mathrm{pH}_{\mathrm{c}}\right)$ of the respective coal samples. Thus, the extended DLVO theory and the calculated hydrophobic parameters for the fresh and oxidized Elkhorn No. 3 coals correlate well with the experimental coagulation results.

For the Pittsburgh No. 8 coal, the methylene iodide and water contact angles were found to be $29^{\circ}$ and $64^{\circ}$, respectively. By applying Eqs. [8]-[16], the values of $C, W_{A}^{\text {dd }}$,

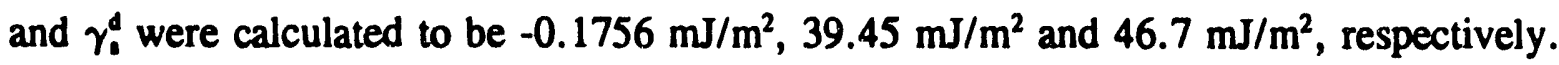
The Hamaker constant for the Pittsburgh No. 8 coal was calculated to be $6.725 \times 10^{-20} \mathrm{~J}$. The zeta-potentials $(\zeta)$ used for determining $V_{R}$ for the Pittsburgh No. 8 sample are shown in Figure 22. These results indicate that the isoelectric point (i.e.p.) for this coal is approximately 3.8. The zeta potential ( () at the critical coagulation $\mathrm{pH}$ of 6.2 was -19.2 . 


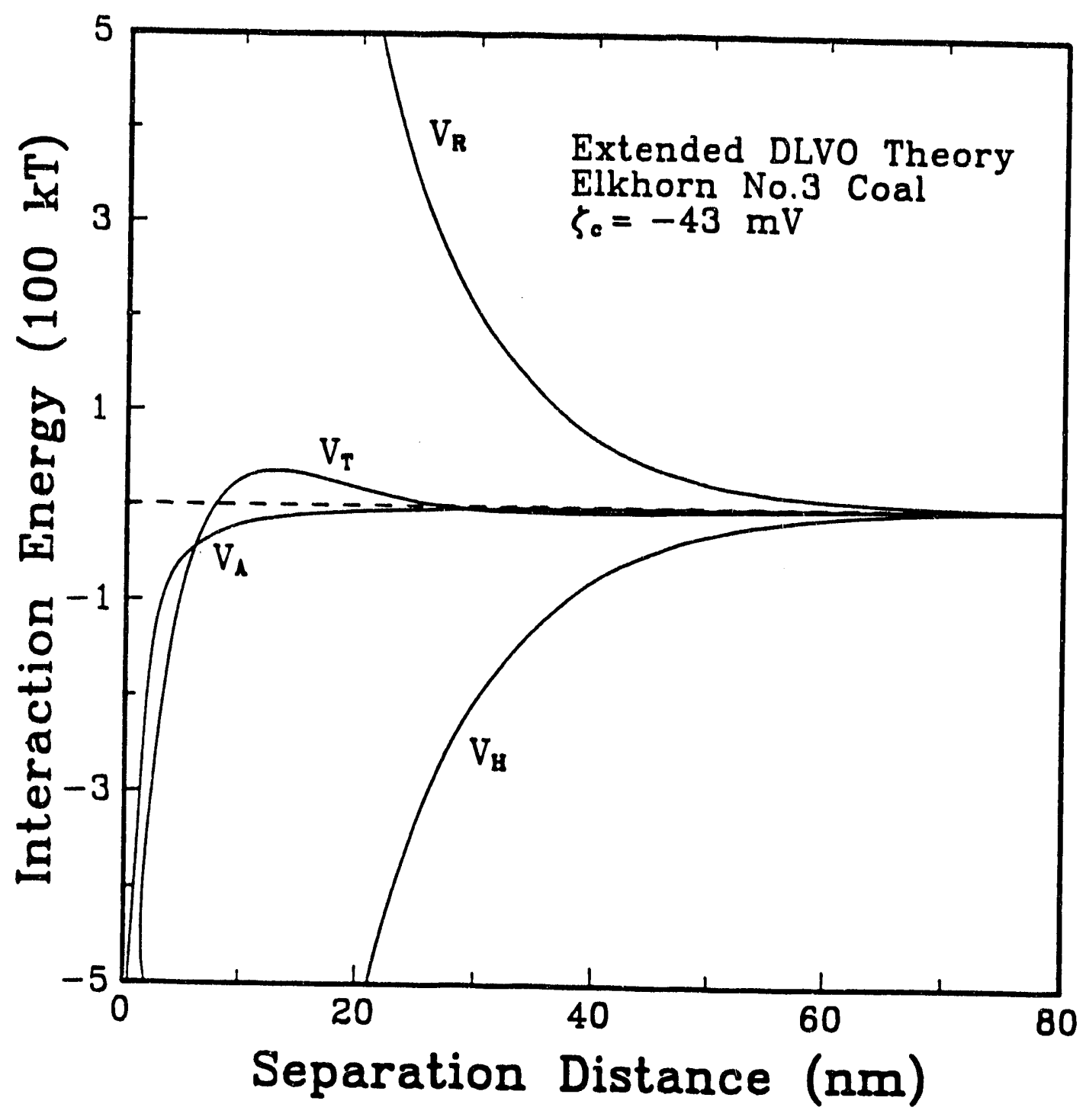

Figure 19. Effect of $\mathrm{pH}$ on the coagulation efficiency and interaction energy barrier of fresh Elkhorn No. 3 coal particles. 


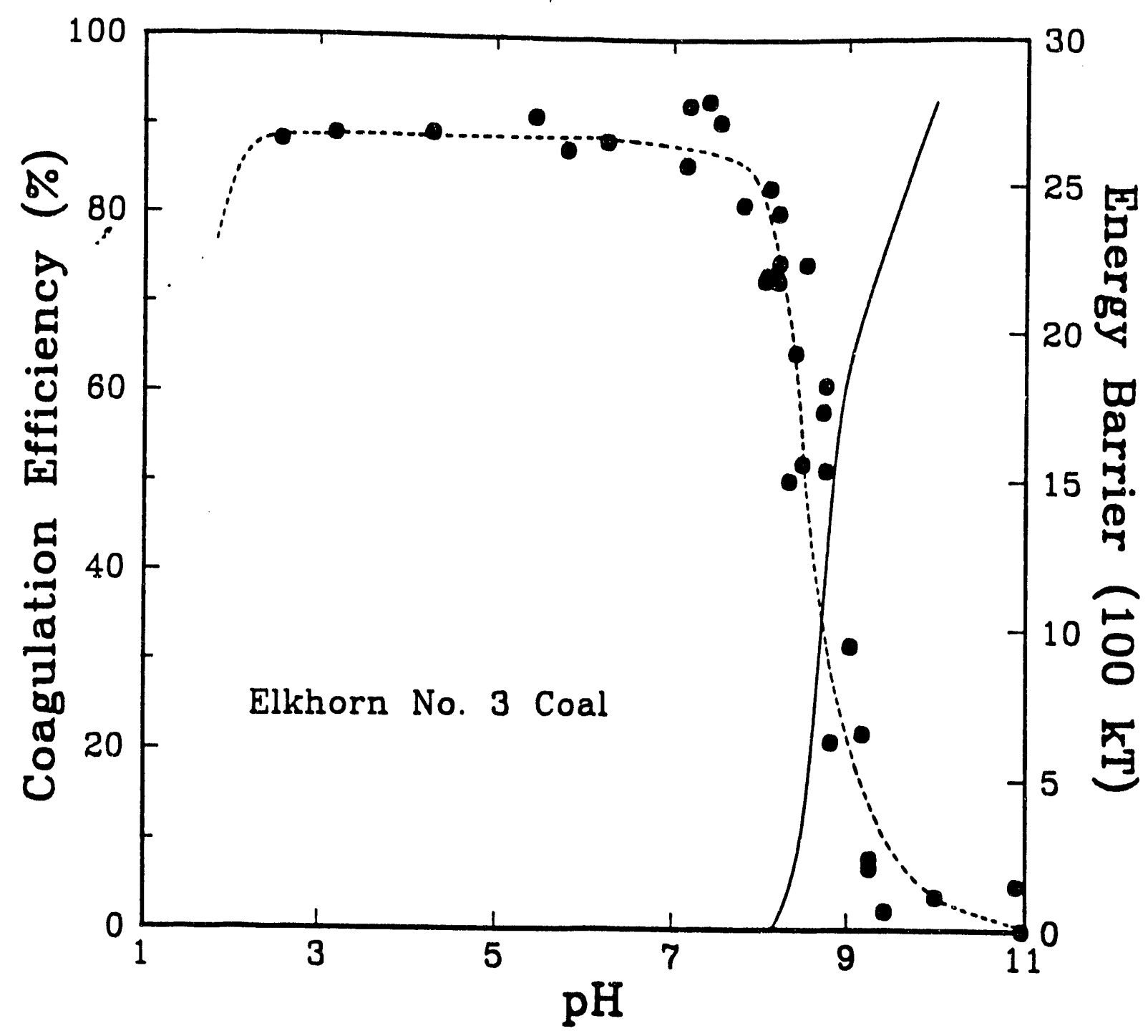

Figure 20. Effect of $\mathrm{pH}$ an the coagulation efficiency and interaction energy barrier of partially oxidized Elkhorn No. 3 coal particles. 


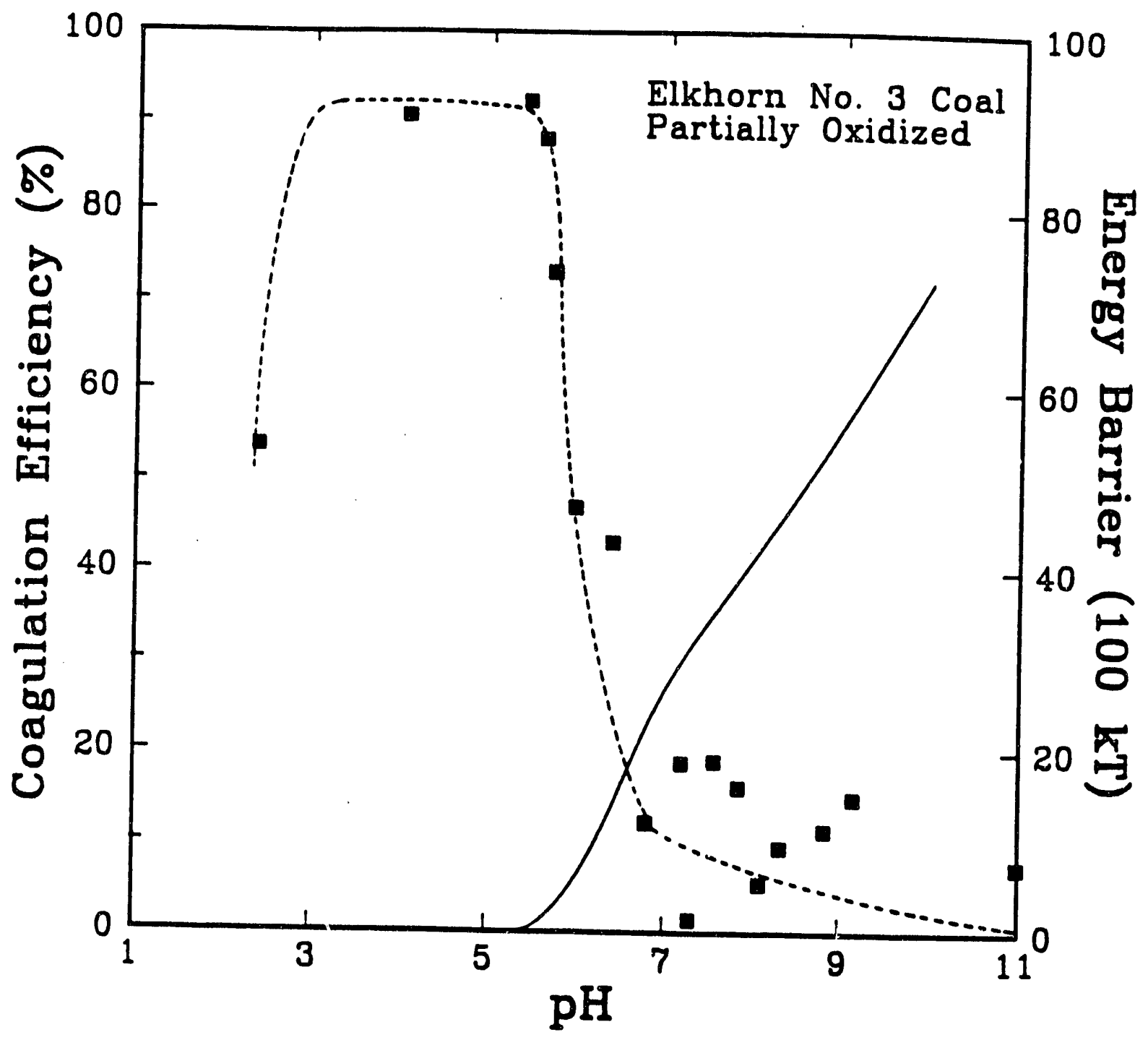

Figure 21. Interaction energy diagram for Elkhorn No. 3 coal particles as calculated from the extended DLVO theory. 


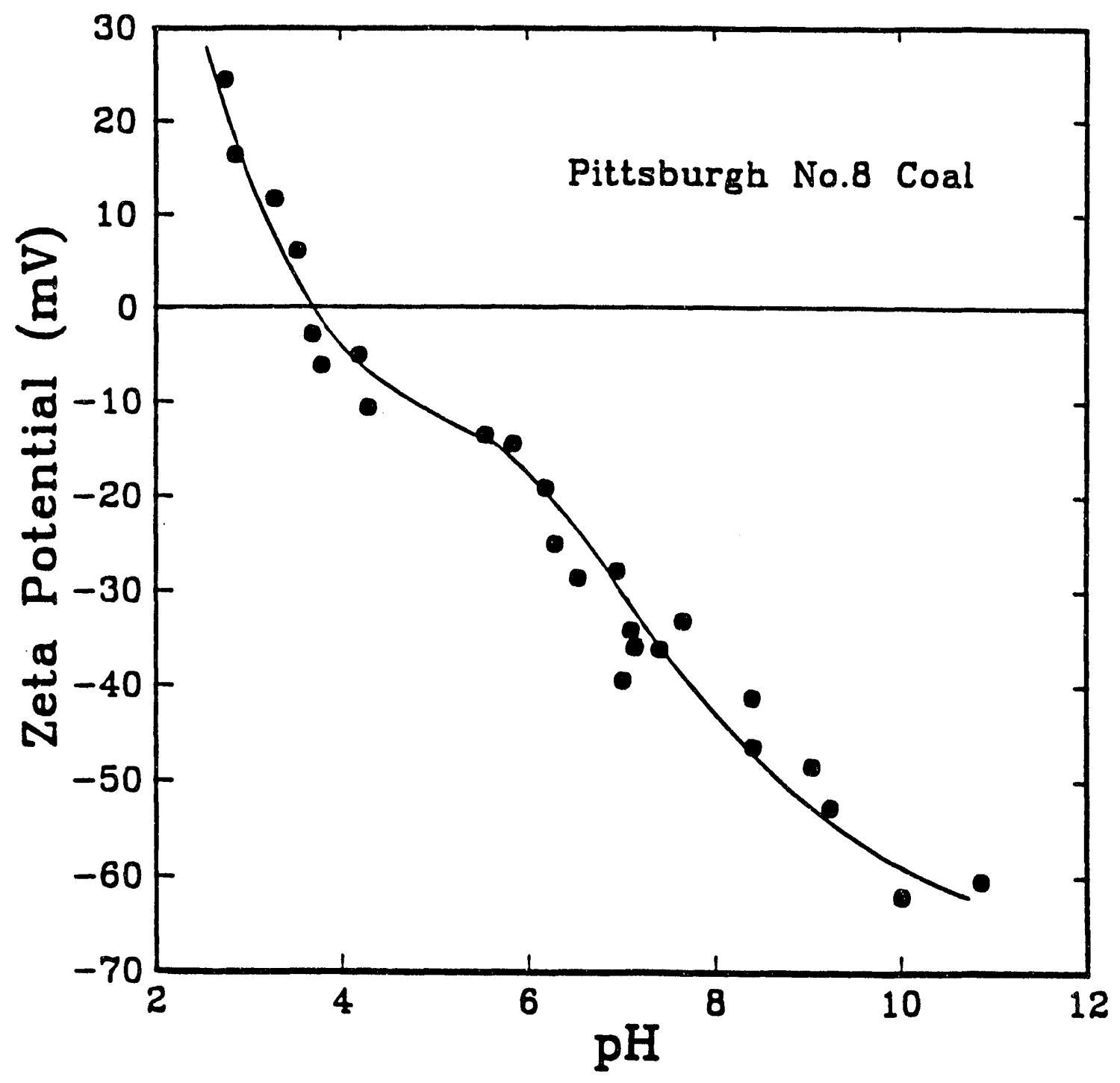

Figure 22. Effect of pH on the zeta potential $(\zeta)$ of Pittsburgh No. 8 coal. 
Utilizing Eqs. [4], [5] and [7], an interaction energy diagram showing the contributions of the various surface forces toward the total interaction energy $\left(V_{T}\right)$ was drawn at the $\mathrm{pH}_{\mathrm{c}}$ of the Pittsburgh No. 8 coal. As shown in Figure 23, the electrostatic and hydrophobic energies are the dominating interactions. The maximum total interaction energy $\left(V_{\mathrm{T}, \max }\right)$ or energy barrier was $66 k \mathrm{~T}$. Coagulation at such a small energy barrier can be induced by a inertia forces and/or gentle mechanical agitation. After obtaining zeta potential $(\zeta)$ values over a range in $\mathrm{pH}$ of $2-11$, the $V_{\mathrm{T}, \max }$ was calculated for each $\mathrm{pH}$ condition and compared with the coagulation efficiency data as shown in Figure 24. The plot shows that as the energy barrier began to increase the coagulation efficiency decieased sharply. Thus, the modified coiloid stability model and the experimental coagulation results also correlate very well for the Pittsburgh No. 8 sample.

\section{Task 2.3 Modeling of Hydrophobic Coaqulation}

A population balance model for describing the hydrophobic coagulation process has been developed. The model utilizes a balance between the rate of particle aggregation and the rate of particle-particle breakage. The aggregation rate $\left(A_{i j}\right)$ is determined from the collision frequency $\left(N_{\mathrm{ij}}\right)$ between particles of type $i$ and $j$. The value of $N_{i j}$ is estimated from the hydrodynamics of mixing and the adhesion efficiency ( $\left.E_{2}\right)$ for particle coagulation. The hydrodynamics of mixing determines the amount of kinetic energy being applied toward particle-particle collision and attachment. The method used to calculate the kinetic energy is outlined in the following discussion along with an example using a silica sample having an average particle size of $3.2 \mu \mathrm{m}$.

The equation proposed by Chia and Somasundaran (1983) was used to determine the viscosity of a concentrated suspension: 


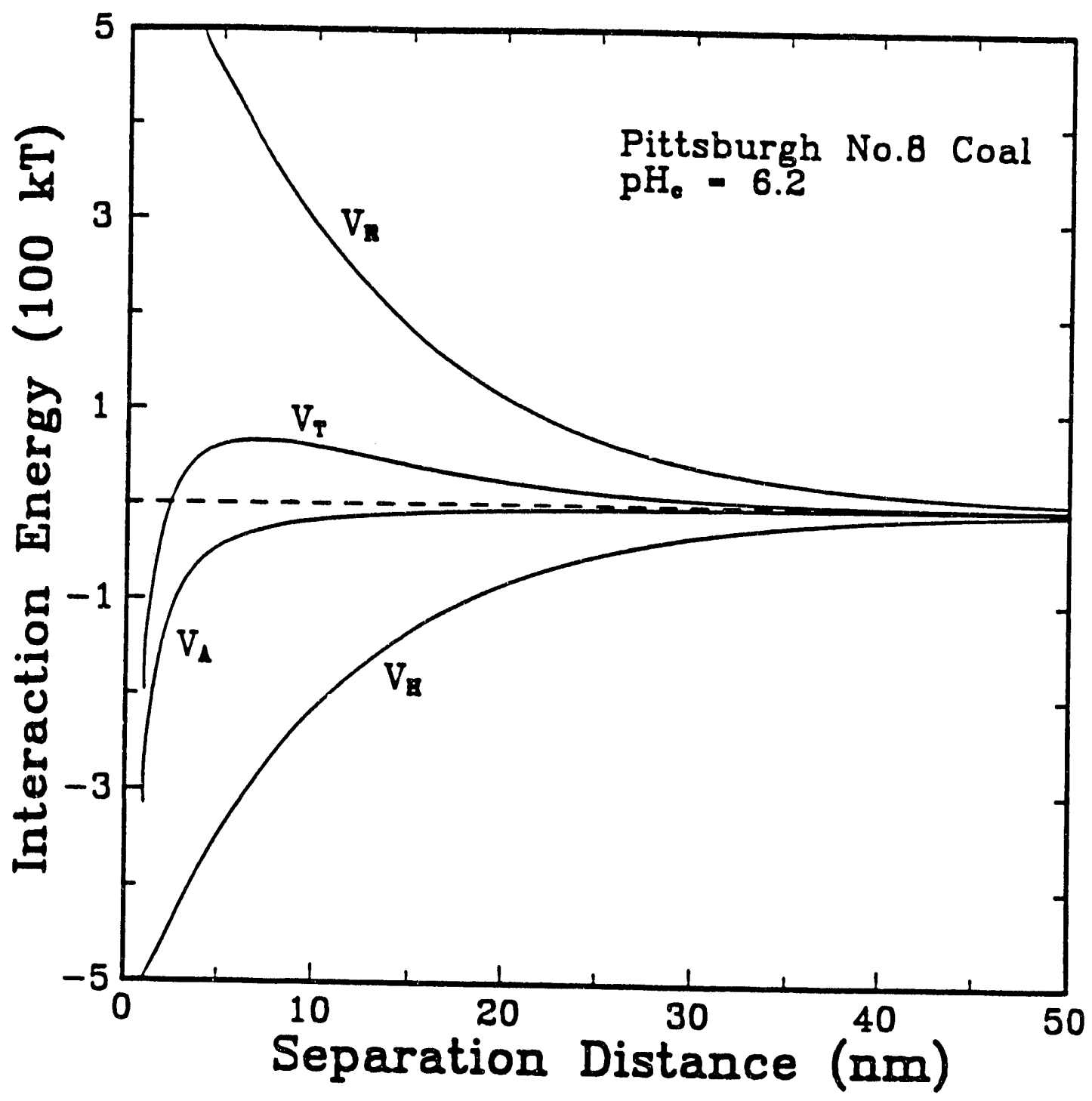

Figure 23. Calculated total interaction energy $\left(V_{\tau}\right)$ as a function of particle separation distance for Pittsburgh No. 8 coal. 


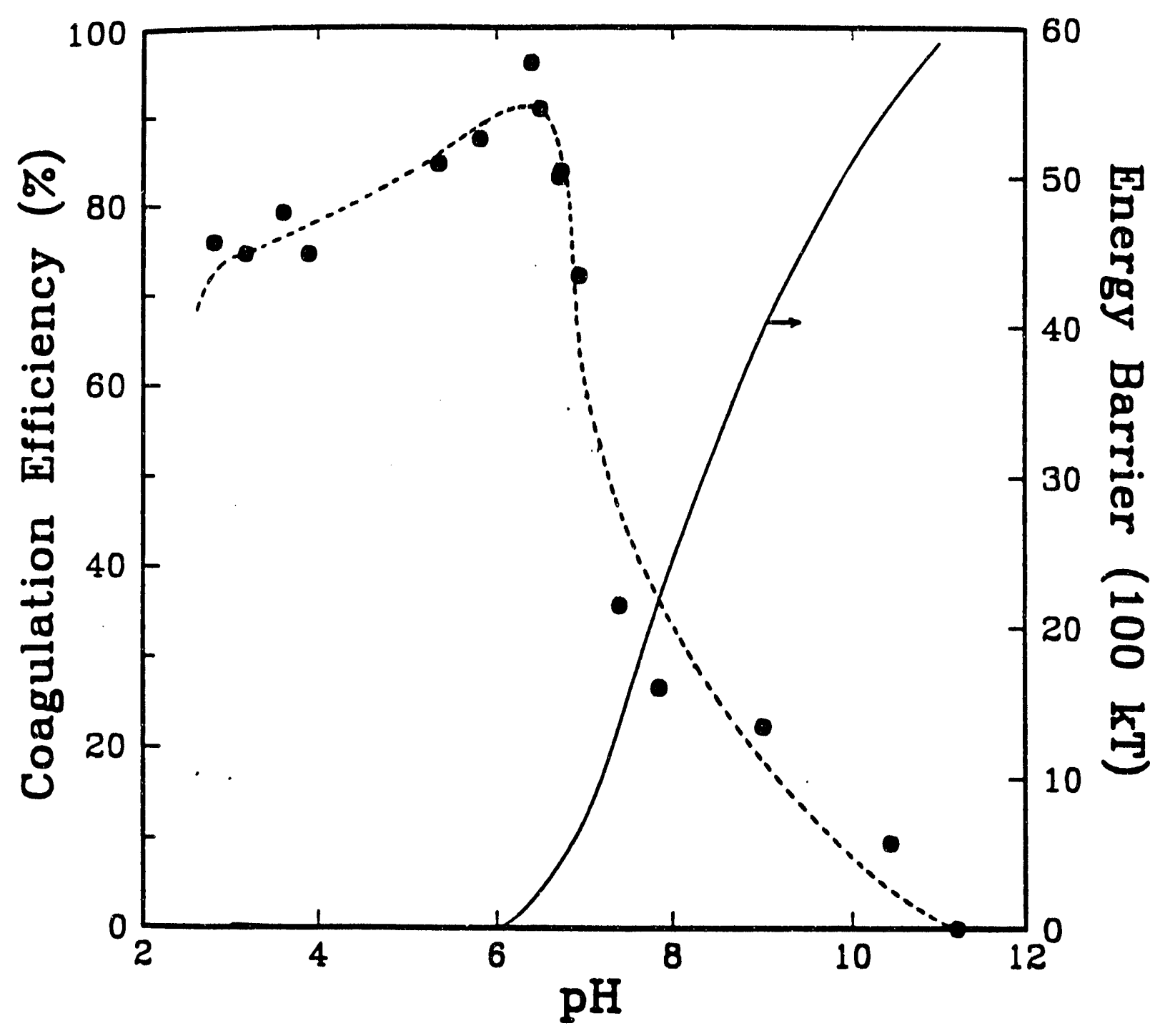

Figure 24. The effect of pH on Coagulation Efficiency and the magnitude (calculated) of the energy barrier for Pittsburgh No. 8 coal. 


$$
\frac{\eta}{\eta_{l}}=1+2.5 \bar{C}+10 \overline{C^{2}}+0.62 \exp \left(\frac{1.875 \bar{C}}{1-1.595 \bar{C}}\right)
$$

in which

$$
\bar{C}=\frac{\left(W_{s} / P_{s}\right)}{\left(W_{s} / P_{s}\right)+\left(W_{l} / P_{l}\right)}
$$

and where $\eta$ and $\eta_{1}$ are the viscosities of the suspension and liquid, respectively, $W_{1}$ and $W_{1}$ are the weights of solid and liquid, respectively, and $\rho_{1}$ and $\rho_{1}$ are the densities of the solid and liquid, respectively.

In the case of the silica example:

$$
\begin{aligned}
& W_{1}=5 \mathrm{gm} \\
& W_{1}=500 \mathrm{gm} \\
& \rho_{1}=2.63 \mathrm{gm} / \mathrm{cm}^{3} \\
& \rho_{1}=1.00 \mathrm{gm} / \mathrm{cm}^{3} \\
& \eta_{1}=0.0089 \text { poise }(\mathrm{gm} / \mathrm{cm}-\mathrm{sec}) .
\end{aligned}
$$

The calculated $\bar{C}$ is 0.00379 and the viscosity of suspension is 0.0145 poise.

The effect of mixing cell geometry, especially the number and size of the baffles, is important in determining the intensity of the mixing environment. The conditional expression used to check for fully baffled conditions is:

$$
(B / D)^{1.2} \times n_{B}<0.35 \text {, }
$$

where $B$ is the width of the baffles, $D$ is the diameter of the container and $n_{\mathrm{B}}$ is the number of baffles. If the calculated value is less than 0.35 , the partially baffled condition should be used in calculating power consumption. For the example, the baffle width, container diameter and the number of baffles are $0.9 \mathrm{~cm}, 8.9 \mathrm{~cm}$ and 4 , respectively. 
Since the calculated result is 0.26 , a partially baffled condition will be used for the calculations.

The critical Reynolds number $\left(\boldsymbol{R}_{\mathrm{c}}\right)$ was determined using the expression:

$$
R_{c}=\frac{25}{(b / D)}\left(\frac{d}{D}-0.4\right)^{2}+\left(\frac{b / D}{0.11(b / D)-0.0048}\right),
$$

where $b$ is the width of the impeller and $d$ is the diameter of the impeller. In the example, the impeller width and diameter is $3.2 \mathrm{~cm}$ and $3.9 \mathrm{~cm}$, respectively. Thus, $\boldsymbol{R}_{\mathrm{c}}$ was calculated to be 10.45. The corresponding $Z$ factor (Nagata, 1975; Fig. 1.38) was 0.991 .

The equation representing maximum power consumption is shown below:

$$
N_{\text {max }}=\frac{\bar{A}}{R_{c}}+\bar{B} Z^{\bar{p}}\left(\frac{H}{D}\right)^{(0.35+b / D)},
$$

in which $H$ is the height of the suspension $(8.5 \mathrm{~mm})$, and:

$$
\begin{gathered}
\bar{A}=14+(b / D)\left[670(d / D-0.6)^{2}+185\right], \\
\bar{B}=10^{\left[1.3-4(b / D-0.5)^{2}-1.14(d / D)\right]}, \\
\bar{p}=1.1+4(b / D)-2.5(d / D-0.5)^{2}-7(b / D)^{4} .
\end{gathered}
$$

For the mixer used in this example, the values for $\bar{A}=86.82, \bar{B}=5.266$ and $\bar{p}=$ 2.412. Therefore, the calculated $N_{P_{\max }}=13.29$.

The power number $\left(N_{\mathrm{PB}}\right)$ with partially baffled conditions was estimated by the following equation:

$$
N_{P B}=N_{P \max }-\left(N_{P \max }-N_{P \infty}\right)\left[1-2.9(B / D)^{1.2} n_{B}\right]^{2} \text {, }
$$

where $N_{\text {Poo }}$ is the power number obtained at $R_{\mathrm{c}}$ tending to infinity, i.e., 


$$
N_{P \omega}=\bar{B}(0.6 / 1.6)^{\bar{p}}(H / D)^{(0.35+H / D)} .
$$

For the mixer in the example, $N_{\mathrm{Pow}}$ was calculated to be 0.479 . Hence, $N_{\mathrm{PB}}$ was found to be 12.46 .

According to Nagata (1975), power input $(P)$ is related to the power number $\left(N_{\mathrm{PB}}\right)$ in the following manner:

$$
P=N_{P B} \rho N^{3} d^{5}
$$

where $N$ is the rotational speed and $\rho$ is the density of the suspension. The rate of energy dissipation per unit mass for turbulent conditions can be calculated in the following way:

$$
\bar{\varepsilon}=\frac{P}{M}=\frac{N_{P B} N^{3} d^{5}}{\bar{V}},
$$

where $M$ is the mass and $\bar{V}$ is the volume of the suspension. In the example, the volume was $502 \mathrm{~cm}^{3}$ and, thus, $\bar{\epsilon}$ was calculated to be $1.30 \times 10^{4} \mathrm{~cm}^{2} / \mathrm{sec}^{3}$ for $N=500 \mathrm{rpm}$.

According to $\mathrm{Xu}(1990)$, the relative velocity $\left(U_{\mathrm{r}}\right)$ depends on the turbulent scale and can be calculated from the Kolmogorov theory of isotropic turbulence:

$$
\lambda=\left(v^{3} / \varepsilon\right)^{1 / 2} .
$$

Under the conditions where the particle size is much smaller than the microscale of turbulence, the relative velocity at the moment of collision is given by:

$$
U_{r}=(1 / 15)^{1 / 2}(\bar{\varepsilon} / v)^{1 / 2}\left(a_{1}+a_{2}\right) \text {, }
$$

where $a_{1}$ and $a_{2}$ are the radii of approaching particles. However, if the particle size is larger than the microscale of turbulence, the inertia mechanism is applicable and the relative velocity should be calculated by using the following equation:

$$
U_{r}=1.37 \bar{\varepsilon}^{-1 / 3}\left(a_{1}+a_{2}\right)^{1 / 3} \text {. }
$$


In the example, $\lambda$ was calculated to be $39 \mu \mathrm{m}$, which is much larger than the particle size of $3.6 \mu \mathrm{m}$. Therefore, Eq. [32] was used and $U_{\mathrm{r}}$ was calculated to be $0.0884 \mathrm{~cm} / \mathrm{sec}$.

From the obtained relative velocity of approaching particles, the kinetic energy provided by mixing can be calculated as:

$$
V_{k}=1 / 2 \mu U_{r}^{2},
$$

where $\mu$ is the reduced mass of particles, which is defined by $1 / \mu=1 / m_{1}+1 / m_{2}$, in which $m_{1}$ and $m_{2}$ are the masses of the approaching particles. In this example, $V_{\mathbf{k}}$ was calculated to be $1.26 \times 10^{-13} \mathrm{erg}$ (or $3.05 \mathrm{kT}$ ).

The kinetic energy has been calculated as a function of mixing rate for coal samples of different particle sizes using the procedure discussed. The results are given in Figure 25. It is clearly shown that there exists a logarithmic linear relation between kinetic energy and mixing rate. The kinetic energy provided by mixing increases significantly as the mixing rate increases. At the same mixing rate, the kinetic energy increases with increasing particle size.

It is well known that in order for coagulation to occur, the particles must collide with each other and adhere. Therefore, the aggregation rate depends upon the frequency of collision and the fraction of collisions which result in adhesion. A coagulum is stable only when the adhesion force is larger than the shear force. To describe the coagulation kinetics, both aggregation rate and coagulum breakage rate need to be considered. In this report, the aggregation rate is derived by considering the collision frequency and adhesion efficiency.

For coagulation to occur with intense mixing, the fluid flow can be considered as the only important mechanism for transporting particles if the particles are greater than 1 $\mu \mathrm{m}$. In this case, the collision frequency $\left(N_{i j}\right)$ can be written as: 


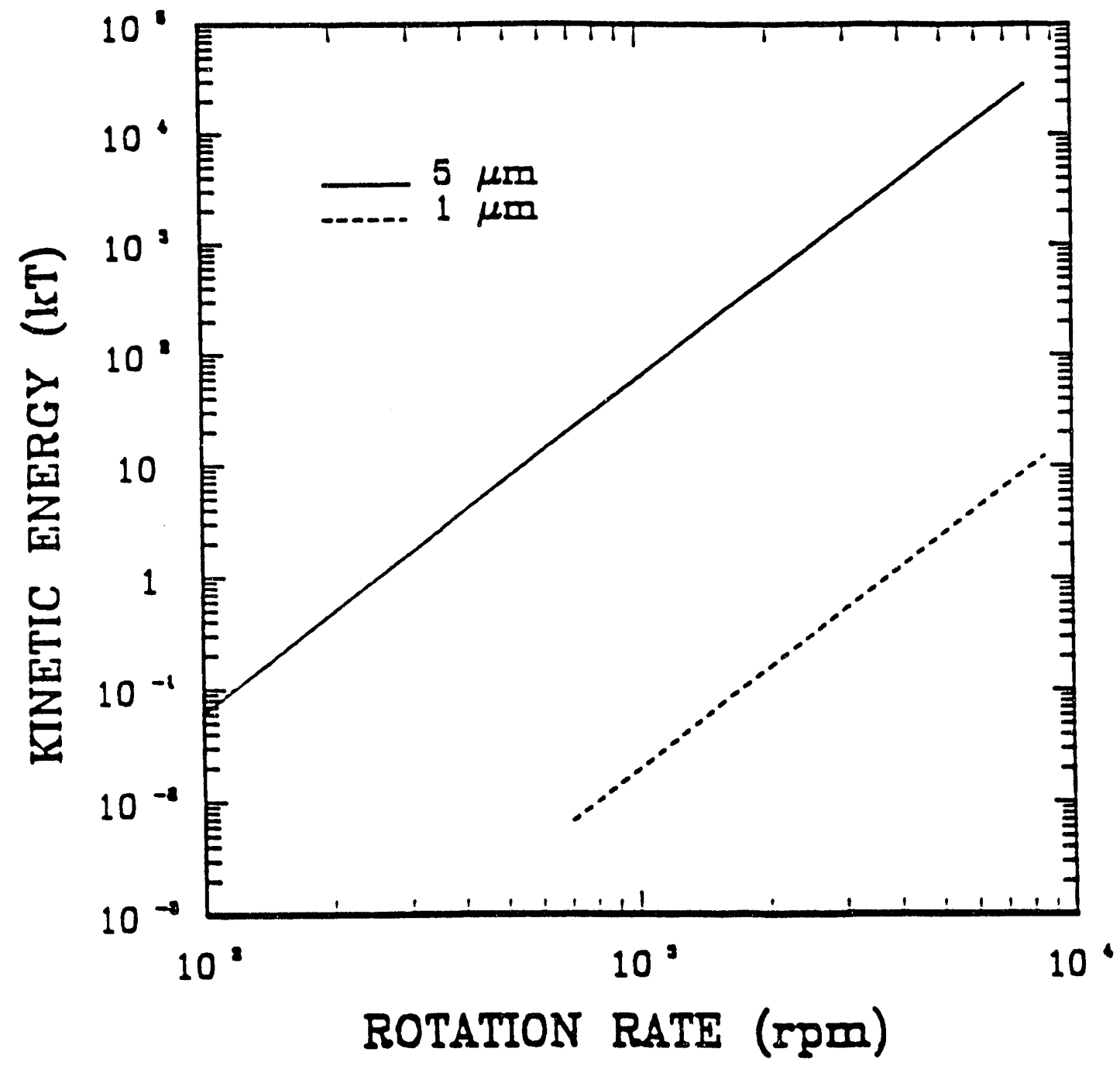

Figure 25. Kinetic energy as a function of mixing rate for coal samples of two different particle sizes. 


$$
N_{i}=\pi\left(a_{i}+a_{j}+H_{m}\right)^{2} U_{i} n(i) n(j),
$$

where $a_{i}$ and $a_{j}$ are the radii of particles and/or aggregates $i$ and $j, n(i)$ and $n(j)$ are the number concentrations of particles $i$ and $j, H_{m}$ is the separation distance between two particles at $V_{T, \text { max }}$, and $U_{i}$ is the relative velocity at a separation distance of $H_{m}$. $U_{i j}$ can be calculated from the hydroc ynamics of the system (as outlined above). It is worth mentioning that when $i=j, 1 / 2$ needs to be inserted into Eq. [35] to prevent double counting of the same particles.

The adhesion efficiency $(\alpha)$ is believed to be a function of both the thermodynamics and hydrodynamics of the system. It is well known that every collision will result in adhesion if the net surface forces between colliding particles are purely attractive. However, if an energy barrier exists due to the repulsive forces, only a fraction of the collisions are effective for adhesion. It is also important to include the hydrodynamics because the kinetic energy of particles can, in some conditions, be large enough to overcome the energy barrier. The adhesion efficiency can, therefore, be considered as unity if the kinetic energy is larger than or equal to the interaction energy barrier. Otherwise, an exponential distribution function can be used as in the following form:

$$
\alpha=\exp \left[-\beta\left(V_{T_{\text {max }}}-V_{k}\right)\right],
$$

in which $\beta$ is an adjustable parameter with units of $(k T)^{-1}$, describing the rate at which the adhesion efficiency decreases with increasing $\left(V_{T, \text { max }}-V_{k}\right)$, where $V_{T, \text { max }}$ is the energy barrier and $V_{k}$ is the relative kinetic energy of the particles at the moment of collision. Details on the calculation of $V_{k}$ were given above.

It is interesting to note that if $V_{k}$ and $\beta$ are set at zero and one, respectively, the above definition of the adhesion efficiency is equivalent to the classical stability ratio 
where the kinetic energy of particles has been ignored. They differ only by a factor of $\lambda\left(a_{1}+a_{2}\right)$, where $\lambda$ is the reciprocal double-layer thickness. It is evident that the classical stability ratio predicts an adhesion efficiency of $\lambda\left(a_{1}+a_{2}\right)$ instead of unity which is expected for unstable suspension having $V_{T, \max }$ equal to zero. Therefore, the adhesion efficiency proposed in the present work seems more reasonable, particularly when the coagulation is conducted under mixing conditions.

Figure 26 shows the dependence of adhesion efficiency on $\left(V_{T, \max }-V_{k}\right)$ as calculated using the present definition. It can be seen that over the range of $V_{T, \text { max }}>V_{k}$, collision efficiency is very sensitive to the interaction energy barrier through its exponential dependence. The sensitivity can be controlled by adjusting the value of the parameter $\beta$. The value of $\beta$ may be obtained by measuring initial coagulation rates experimentally under conditions where breakage of aggregates can be neglected.

By combining frequency and adhesion efficiency, the overall aggregation rate constant $\left(A_{i}\right)$ can be expressed as:

$$
A_{i j}=\alpha \pi\left(a_{i}+a_{j}+H_{m}\right)^{2} U_{i j} .
$$

To calculate the aggregation rate constant, it is necessary to know the interaction energy barrier due to the surface forces. By using the extended DLVO theory as developed in Task 2.2 of the present work, various components of particle-particle interactions and, hence, the energy barrier, $V_{T, \text { max }}$, can be calculated if the surface and solution characteristics, such as zeta-potentials, Hamaker constant, non-dispersion component of work of adhesion of water and the ionic strength of the slurry, are known. These parameters can be obtained experimentally. Combined with the hydrodynamic conditions, one can predict the aggregation rate constant and control it by adjusting the physico-chemical and hydrodynamic conditions of the system. 


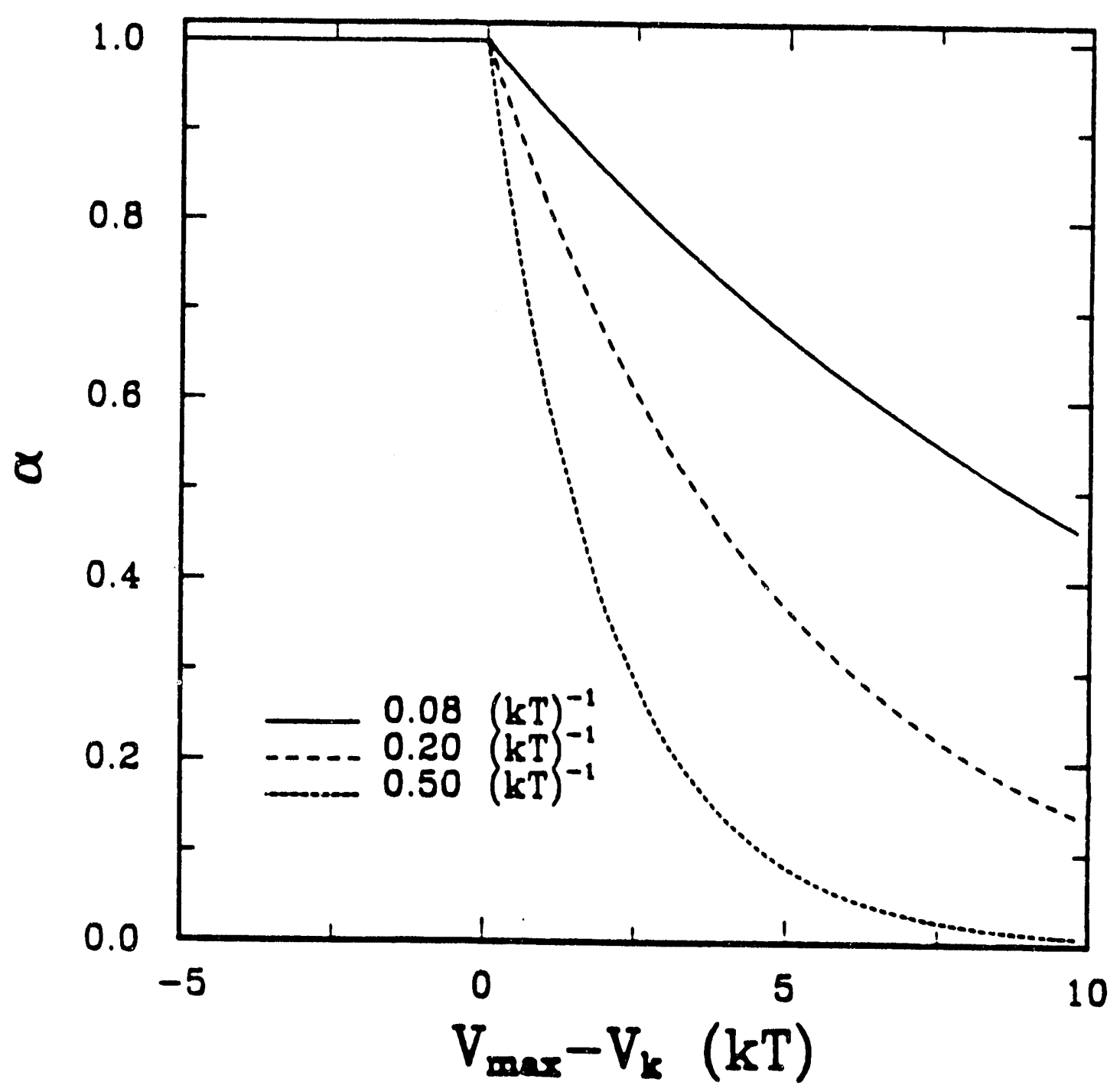

Figure 26. Comparison of collision efficiency for three different values of model parameter $\beta$. 
It is also well known that under turbulent conditions, the aggregates are subjected to shear forces. If the shear force is greater than the adhesion force holding the particles together as an aggregate, the aggregate will break into small fragments. Therefore, it is also necessary to consider the breakage event in forming the coagulation kinetic equation.

In general, intense mixing promotes agglomeration by increasing the particleparticle collision frequency. At the same time, collision efficiency will also be improved due to the high level of kinetic energy provided to the interacting particles. If the kinetic energy is high enough to overcome the interaction energy barrier, every collision will result in agglomeration. However, if the mixing is too vigorous, the shear force produced may exceed the binding force of the agglomerate, resulting in breakage. Thus, a successful coagulation model must consider both the agglomeration and breakage rate. The rate of breakage will be derived by considering both the binding force of the agglomerate and the shear force.

When two or more particles form an aggregate, there is a reduction in the surface/water interface due to the direct contact of particles. The binding force is, therefore, a function of the solid/liquid interfacial tension and contact area. The former can be evaluated from contact angle measurements while the latter is a function of the particle shape, the number of particles composing the aggregate, and the structure of the aggregate. In general, the magnitude of the binding force, $F_{b}$, holding the agglomerates together is a product of twice the net contact area and the interfacial tension and is given by:

$$
F_{b}=2 \gamma_{s w} A_{n},
$$

where $\gamma_{s w}$ is the solid/water interfacial tension and $A_{n}$ is the net contact area. Since our knowledge of the relationship between contact area and aggregate size is very limited, an 
assumption has been made that the net contact area is proportional to the cross-sectional ared of the aggregate, i.e.,

$$
A_{n}=k_{1} a^{2}(1-\varepsilon)^{2 / 3}
$$

where $\varepsilon$ is the porosity of the aggregate, $a$ the radius of the aggregate, and $k_{l}$ is an adjustable parameter. The value of $k_{1}$ represents the mechanical properties of the particles, such as elasticity, particle shape, and structure of the aggregate. Substituting Eq. [38] into Eq. [39] results in the following equation which can be used for evaluating the binding force:

$$
F_{b}=2 k_{1} \gamma_{s w} a^{2}(1-\varepsilon)^{2 / 3}
$$

The binding force, $F_{b}$, has been calculated as a function of aggregate size for various values of $k_{1}$. The results shown in Figure 27 indicate that $F_{b}$ increases with increasing aggregate size. This result is obvious since increasing aggregate size results in an increase in the total contact area. Figure 27 also indicates that $F_{b}$ is very sensitive to the value of $k_{1}$. Since $k_{1}$ is a function of aggregate structure (which is affected by hydrodynamics), it is necessary to quantify $k_{1}$ for each system under a given set of operating conditions in order to obtain an accurate estimation of $F_{b}$.

In contrast to the binding force, the shear force is the major contributor to aggregate breakage. The shear force, $F_{s}$, acting on a unit area on opposite sides of the aggregate is proportional to the average value of the velocity fluctuations of the two sides and is given by:

$$
F_{s}=k_{2} A_{s} p_{w} U_{a}^{2}
$$

where $k_{2}$ is a proportionality constant, $A_{s}$ the cross-sectional area of shear, and $U_{a}$ is the average value of the velocity fluctuations evaluated at a sefaration distance $a$. The value of $U_{\text {a }}$ can be determined using the Kolmogorov theory of isotropic turbulence. The 


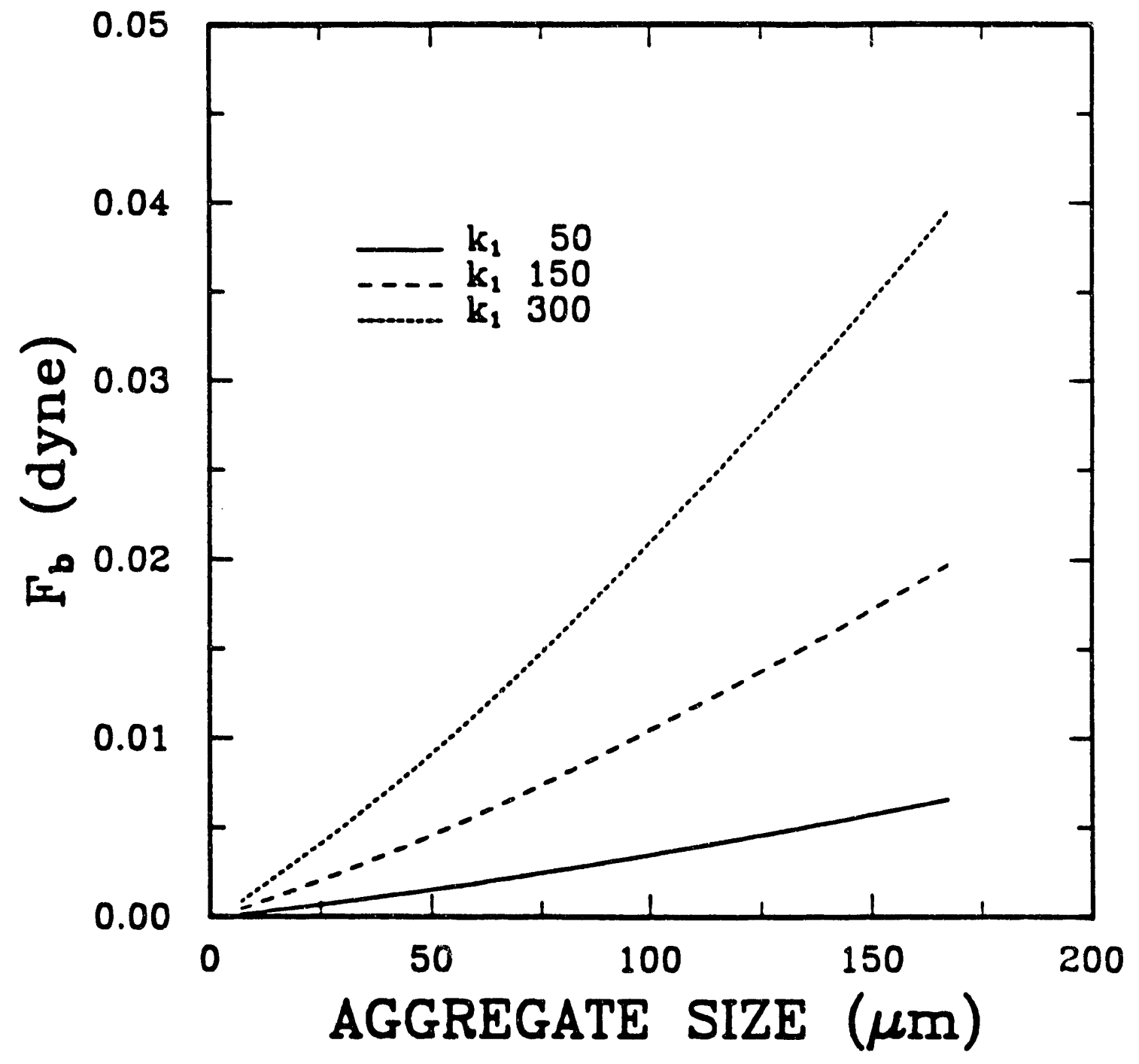

Figure 27. Variation of binding force as a function of aggregate size for three different values of model parameter $k_{1}$. 
equations used to calculate $U_{a}$ has been discussed above.

By using Eq. [41], the shear force has been calculated as a function of aggregate size for different agitation speeds. The system studied was a $5 \%$ by weight coal suspension with the primary particles being $5 \mu \mathrm{m}$ in diameter. As shown in Figure 28, the test results obtained by using the value of unity for $k_{2}$ show that the shear force increases with increasing aggregate size. The aggregate size dependence is greater under high agitation conditions. The results also show that, for a given aggregate size, the shear force increases dramatically as shear intensities increase. This finding indicates that too vigorous mixing will break the aggregates that are relatively small in size and reduce the aggregate top size.

In the breakage model, the aggregate will break into a spectrum of daughter aggregates/particles if the shear force is greater than the binding force. The breakage rate constant, $S(a)$, is defined as such that $S(a) n(a) d t$ is the number of aggregates having size $a$ that split per unit volume in the infinitesimal time interval $d t$. The following functional form was used for describing the breakage rate constant:

$$
\begin{array}{ll}
S(a)=0 & \text { if } F_{b}>F_{s} \\
S(a)=A\left[1-\exp \left[k_{3}\left(F_{b}-F_{g}\right)\right]\right] & \text { if } F_{b} \leq F_{z}
\end{array}
$$

where $S(a)$ has units of $\sec ^{-1}$. The constants $A$ and $k_{3}$ are adjustable parameters which can be determined by fitting experimental data to the simulation results. Eq. [42] simply means that breakage does not occur if $F_{b}$ is greater than $F_{s}$. If $F_{b}$ is less than $F_{s}$, the breakage rate is exponentially proportional to the net breakup force $\left(F_{b}-F_{s}\right)$.

The behavior of this breakage rate function is illustrated in Figure 29 as a function of aggregate size. In the calculation of breakage rates, Eqs. [40]-[42] have been used in combination. The calculations have been conducted for a system having a solids 


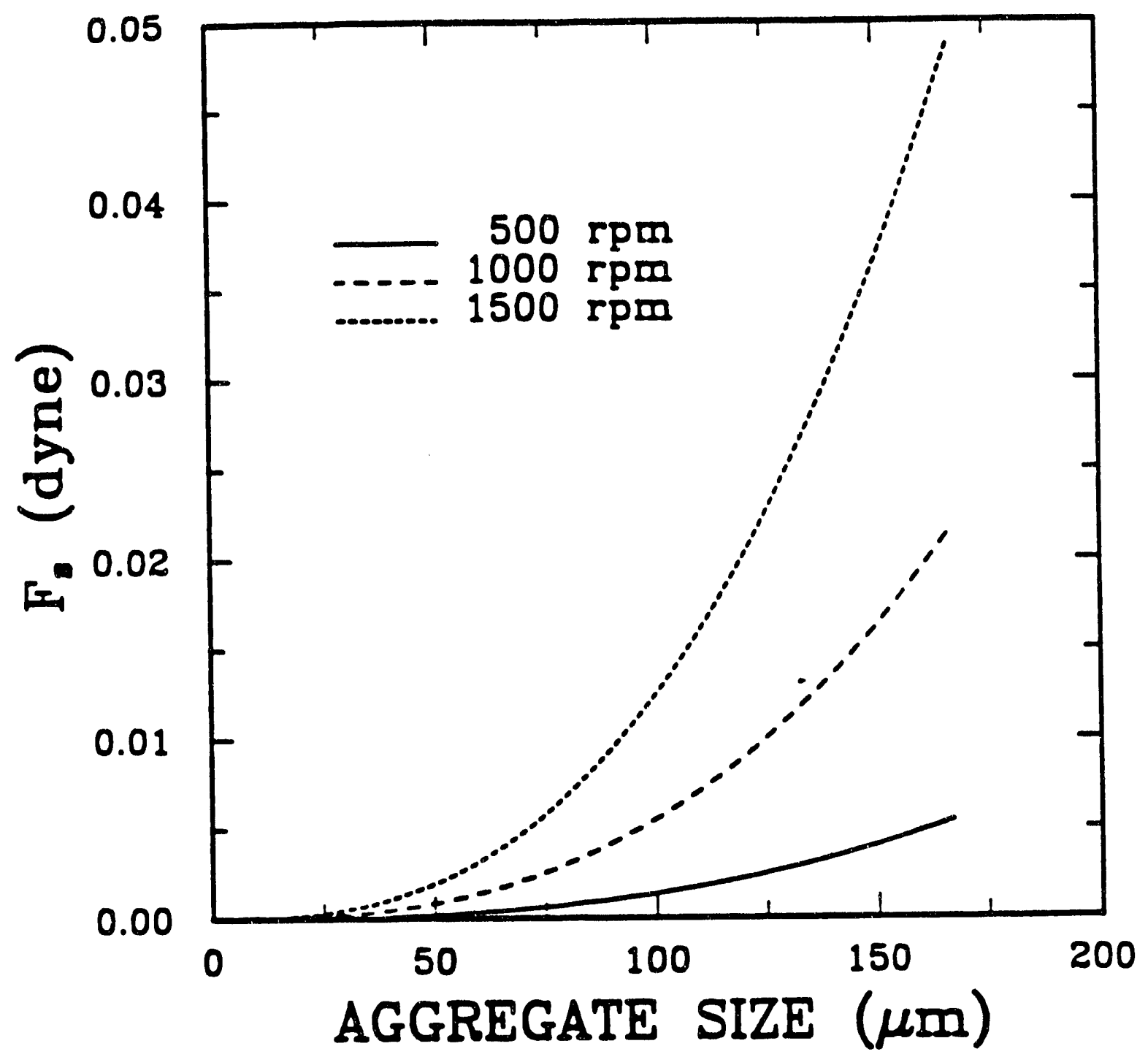

Figure 28. Variation of shear force as a function of aggregate size for three different mixing rates. 


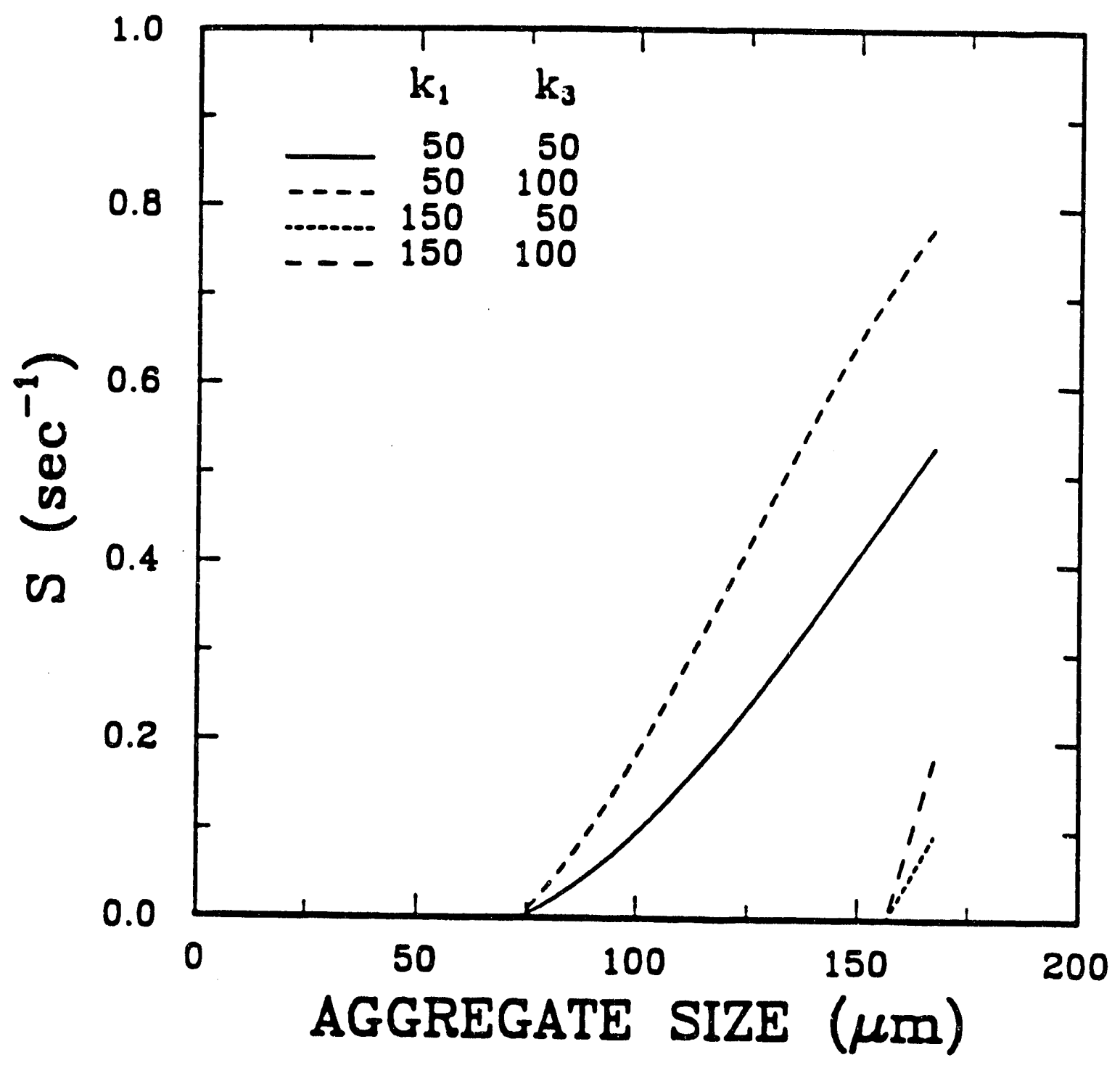

Figure 29. Variation of breakage rate constant as a function of aggregate size for different values of model parameters $k_{1}$ and $k_{3}$. 
concentration of $5 \%$ by weight of $5-\mu \mathrm{m}$ coal conditioned at $1000 \mathrm{rpm}$. For sake of simplicity, $k_{2}$ was assumed to be equal to unity. This did not effect the breakage rate constant since $k_{2}$ can be pulled out, resulting in new values for $k_{1}$ and $k_{3}$. For convenience, $A$ was also given a value of unity for these calculations.

Figure 29 indicates that breakage will not occur until the aggregate grows to a certain size. The size at which the aggregate begins to break increases significantly as $k_{1}$ increases. This finding suggests that compact aggregates of soft material can be subjected to a higher shear field without breakage, i.e., forming aggregates of larger size due to the larger value of $k_{1}$. Figure 29 also shows that by assuming the same value of $k_{1}$, the breakage rate increases substantially with an increase in $k_{3}$. However, the aggregate size at which the aggregates start to break does not change.

In order to study the influence of shear force on aggregate breakage, breakage rate constants were calculated over a range of aggregate size for $1000 \mathrm{rpm}$ and $1500 \mathrm{rpm}$. The values of $k_{1}$ and $k_{3}$ were kept constant at 150 and 100, respectively. The results given in Figure 30 show that not only the breakage rate but also the size corresponding to the beginning of aggregate breakage changes as the mixing intensity is increased. This finding suggests that mixing intensity is a most sensitive adjustable operation variable to control the ultimate aggregate size and its distribution.

The above analyses indicate that this simple three-parameter $\left(k_{1}, k_{3}, A\right)$ model seems appropriate for describing the breakage rate. The values of these three parameters can be determined by curve-fitting with experimental results. In order to complete the description of the breakage cvent, the distribution function of the fragments resulting from the breakage of larger aggregates, known as the breakage function, must also be determined. 


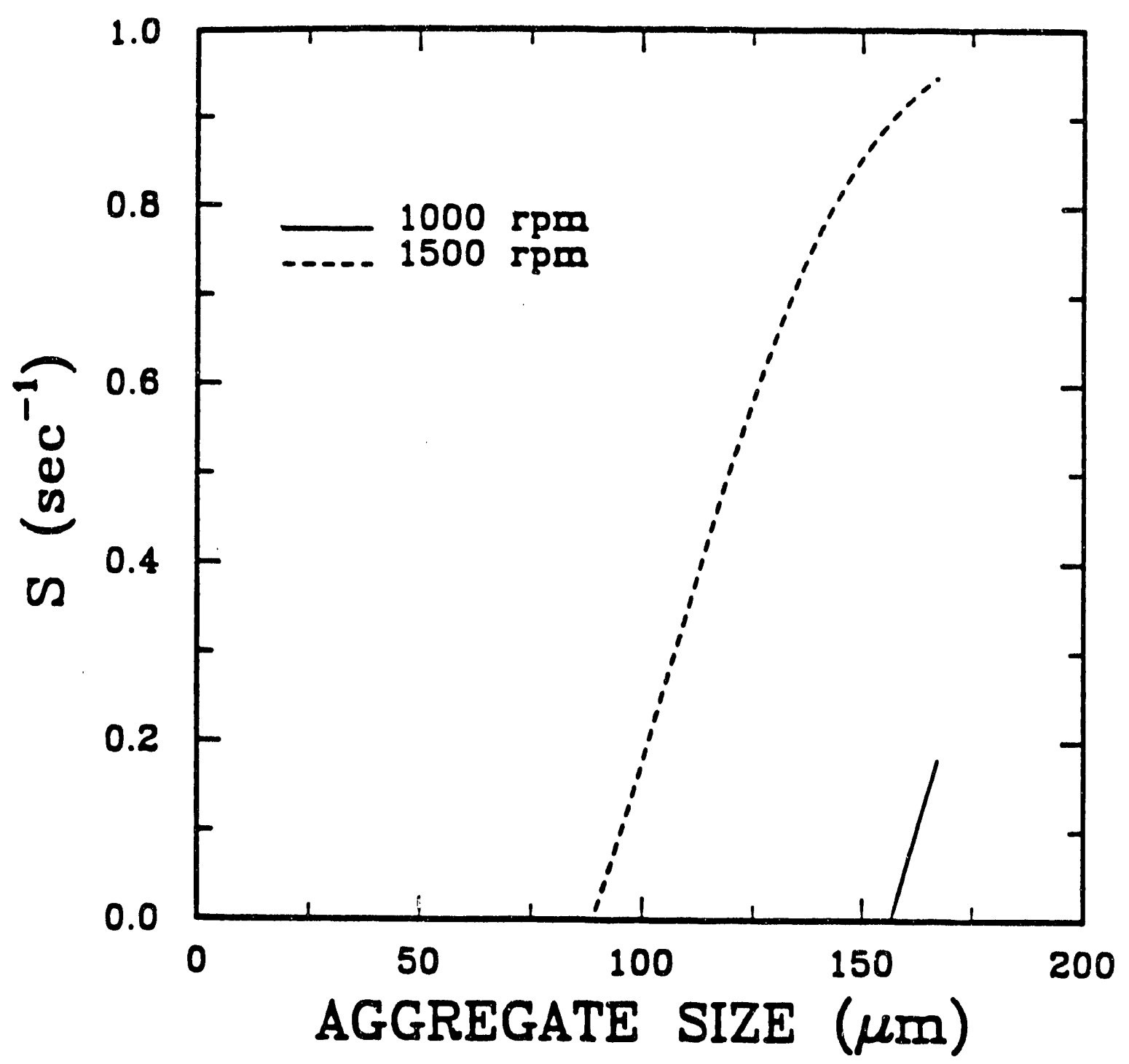

Figure 30. Variation of breakage rate constant as a function of aggregate size for two different mixing rates. 
Upon breakage, an aggregate of size $a$ turns into a number of daughter fragments which are distributed in the size space according to a distribution function, $B\left(a^{\prime}, a\right)$. $B\left(a^{\prime}, a\right)$ is the number fraction of aggregates of size $a$ that report into a size class of $a^{\prime}$ after their breakage. In the present work, the Broadbent and Callcott breakage function (Broadbent and Callcott, 1956, 1957) was used without considerable modifications to describe the aggregate breakage function. For convenience, the aggregate number instead of aggregate size is used in the following:

$$
B\left(z^{\prime}, z\right)=\frac{e^{-\left(z^{\prime}-1\right) / z}-e^{-z^{\prime} / z}}{1-e^{-1}} \frac{z}{z^{\prime}},
$$

where $z$ and $z^{\prime}$ refer to the $z$ th and $z^{\prime}$ th aggregates, respectively. $B\left(z^{\prime}, z\right)$ represents the fractional number of $z$ th aggregates formed by the breakage of a $z$ th aggregate. It should be noted that the factor $\left(z / z^{\prime}\right)$ which occurs in Eq. [43] represents the fact that every zth aggregate produces $\left(z / z^{\prime}\right) z^{\prime}$ th daughter aggregates if all daughter aggregates are of the same aggregate number, $z^{\prime}$. Apparently, the following relation is satisfied for the present breakage function:

$$
\sum_{z^{\prime}=1}^{\infty} B\left(z^{\prime}, z\right)=\sum_{z^{\prime}-1}^{z-1} B\left(z^{\prime}, z\right)=z
$$

The first equality follows from:

$$
B\left(z^{\prime} z\right)=0 \quad z^{\prime} z z,
$$

indicating that all daughter aggregates must be smaller than the parent aggregate. Eq. [45] implicitly assumes that aggregates of a given size do not break into their own class, which is a sound assumption if the primary particles are mono-sized and the spectrum of aggregate size is classified in such a way that the aggregate in each successive class contains one more primary particle than the aggregate in the previous class. 
From the development of the aggregate growth model discussed above and the breakage model in the above discussion, it is possible to develop a general population balance model to describe the dynamic behavior of coagulation under mixing. The general conservation equation used in the development of the dynamic population balance model can be expressed as:

$$
\text { Accumulation }=\text { Input }- \text { Output }+ \text { Net Generation } \text {. }
$$

Aggregate size is the only property considered in the present population balance model. By applying the general theorem of Eq. [46] to a closed coagulation system, the basic deterministic equation for the number balance of any zth aggregate size class is obtained as follows:

$$
\begin{aligned}
\frac{8 n(z, t)}{\delta t}= & \frac{1}{2} \sum_{i=1}^{t=z-1} R C(z-i, i) n(z-i, t) n(i, t)-n(z, t) \sum_{i=1}^{\infty} R C(z, i) n(i, t) \\
& +\sum_{i=z+1}^{\infty} S(i) B(z, i) n(i, t)-S(z) n(z, t), \quad z=3,4, \ldots,
\end{aligned}
$$

where $n(z, t)$ represents the time dependence for the number concentration of the $z$ th size class, $R C(z, i)$ is the coagulation rate between aggregates in the $z$ th size class and the $i$ th size class, $S(z)$ is the breakage rate of aggregates in the zth size class, and $B(z, i)$ is the breakage function representing the fraction of $i$ th-class aggregates formed by the breakage of a zth-class aggregate.

The first term in Eq. [47] represents the increase in the number concentration of zth sice class aggregates which are formed by binary aggregation of smaller aggregates and/or particles within a specific unit of time. The second term describes the decrease in the number concentration of zth-class aggregates due to coagulation between a zth-class aggregate and any other aggregate. Together, these two terms describe the aggregate 
growth behavior. The third term accounts for the appearance of $z$ th-class aggregates by the breakage of a larger aggregate, and the fourth represents the disappearance of $z$ thclass aggregates due to their breakage into a spectrum of smaller daughter aggregates. Together, the third and fourth terms describe aggregate breakage behavior.

It has been found experimentally that a maximum stable aggregate size exists for each coagulation system. Therefore, the infinity sign in Eq. [47] can be replaced with a maximum aggregate size, $N$. Under this constraint, the application of the general population balance equation to (i) the primary particle, (ii) the dimer aggregate, and (iii) the top aggregate class yields the following equations:

$$
\begin{gathered}
\frac{8 n(1, t)}{\delta t}=-n(1, t) \sum_{i=1}^{N} R C(1, i) n(i, t)+\sum_{i=2}^{N} X(i) B(1, i) n(i, t), \\
\frac{\delta n(2, t)}{\delta t}=R C(1,1) n(1, t)^{2}-n(2, t) \sum_{i=1}^{N} R C(2, i) n(i, t)+\sum_{i=3}^{N} S(i) B(2, i) n(i, t)-S(2) n(2, t), \\
\frac{\delta n(N, t)}{\delta t}=\frac{1}{2} \sum_{i=1}^{N-1} R C(i, N-i) n(i, t) n(N-i, t)-n(N, t) \sum_{i=1}^{N} R C(i, N) n(i, t)-S(N) n(N, t) .
\end{gathered}
$$

In order to complete the description of the closed system, an additional constraint must be imposed on Eqs. [48]-[50]:

$$
\frac{8 \sum_{z=1}^{N} z n(z, t)}{8 t}=0 .
$$

Eq. [51] indicates that the total mass of the solids in a system does not change with time. In integral form, this constraint can be written as: 


$$
\sum_{z=1}^{N} z n(z, t)=n(1,0)
$$

The result of the above analysis is a macroscopic population balance model that describes both the aggregate growth and aggregate breakage events. It is interesting to note that if the breakage event is neglected in Eq. [47], the model simplifies to the discrete form of the well-known Smoluchowski's coagulation equation. Therefore, the present model can be applied to more general coagulation systems than can the model developed by Smoluchowski, which only accounts for special cases.

In this study, the population balance model equation was developed for 500 individual size fractions $(N=500)$. After predetermination and/or calculation of all parameters and constants, the equations were solved numerically using the backward differentiation formula known as Gear's stiff method and a Jacobian matrix. The solution procedure is summarized in the schematic flow chart shown in Figure 31.

In order to validate the model developed in the present work, the predicted aggregate size distribution was compared with that of an aggregate generated from a -400 mesh Elkhorn No. 3 coal sample. Aggregate sizes were estimated from settling rate tests carried out using the Andreasen pipette technique. The simulations were conducted using model parameters determined for the Lower Cedar Grove coal sample at the critical coagulation $\mathrm{pH}$. These parameters were believed to be reasonably applicable to the Elkhorn seam coal because of the similarities between the two coal samples. In the model simulation, an experimentally determined $D_{90}$ value of $16 \mu \mathrm{m}$ was used as the primary particle size and a binary breakage function was used in place of the proposed Broadbent and Callcott function to obtain better agreement between the experimental and simulation results. 


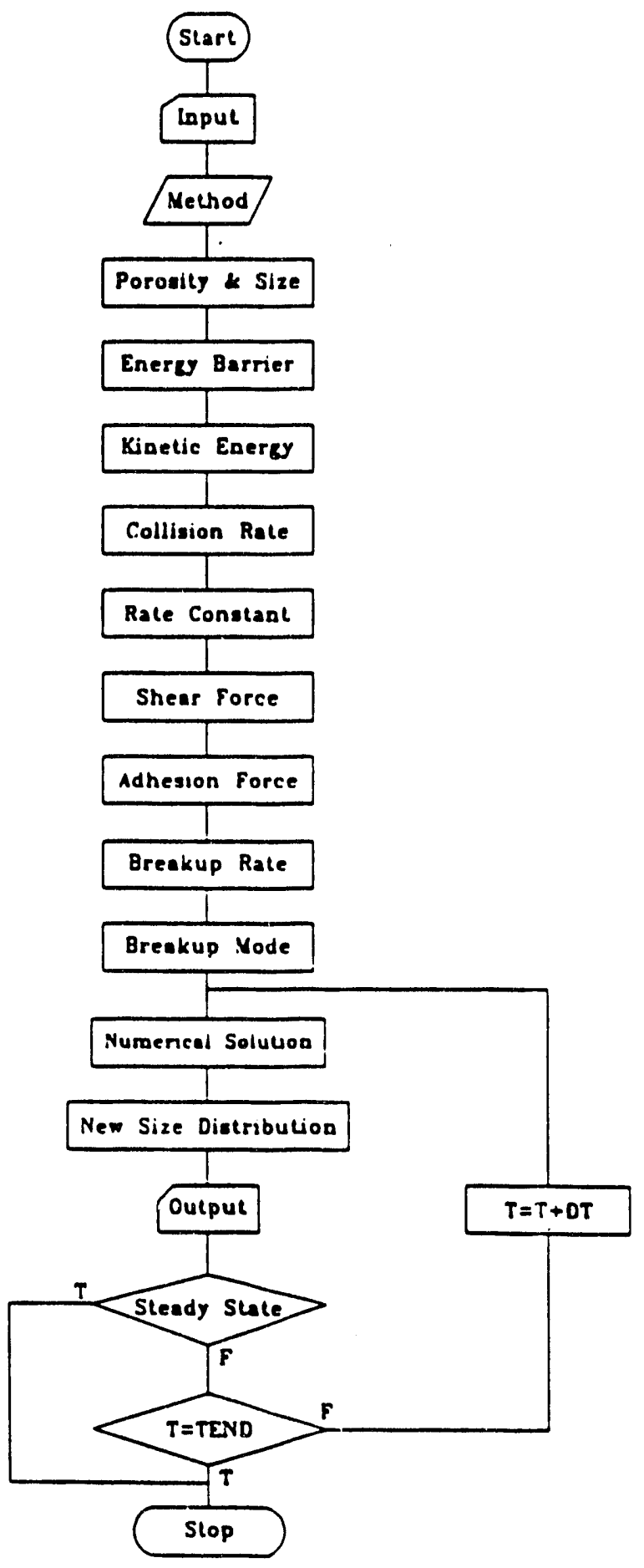

Figure 31. Schematic diagram of the computer program for simulating coagulation kinetic behavior. 
The experimental and predicted aggregate size distributions are shown in Figure 32. The experimentally measured aggregate top size of approximately $50 \mu \mathrm{m}$ is almost identical to that predicted by the model. The lack of agreement in the aggregate size range greater than $50 \mu \mathrm{m}$ may be attributed to inaccuracies in the aggregate size measurements obtained using the Andreasen pipette. It is also possible that the model is unable to predict the size distribution of particles in the tail of the fine size range due to the nature of the binary breakage function used in the simulation.

The effects of varying three of the fundamental parameters used in this study, i.e., the hydrophobicity interaction parameter, $C$; the zeta potential, $\zeta$, and the Hamaker constant, $A_{131}$; are given in Figures 33,34 and 35 . Figure 33 shows that a decrease in the magnitude of the hydrophobic interaction parameter from $-1.247 \mathrm{~mJ} / \mathrm{m}^{2}$ to -1.147 $\mathrm{mJ} / \mathrm{m}^{2}$ (i.e., a decrease in hydrophobicity) caused only minor changes to the equilibrium size distribution, but that the further decrease to $-1.047 \mathrm{~mJ} / \mathrm{m}^{2}$ prevents coagulation from occurring. These relatively small changes in $C$ actually represent significant changes in the interaction energy barrier (from $67.6 k \mathrm{~T}$ to $161.3 k \mathrm{~T}$ and $294.2 k \mathrm{~T}$ ). Similar results were obtained for zeta potential $(\zeta)$, where a change in zeta potential from -43 to $-45 \mathrm{mV}$ caused a change from coagulation to dispersion. Again, this was due to significant changes in the interaction energy barrier (from $67.6 k \mathrm{~T}$ to $188.7 k \mathrm{~T}$ ). Figure 35 demonstrates that the Hamaker constant $\left(A_{131}\right)$ does not materially affect the equilibrium aggregate size distribution. A theoretical analysis of the effect of the Hamaker constant indicates that it is not as important as surface electrical potential in determining the stability of colloidal suspensions of hydrophobic materials. Instead, this parameter mainly affects the kinetics of coagulation, particularly in the early stages of aggregate growth. As aggregate size increases, the kinetic energy of these aggregates tends to determine collision efficiency, and as a result, the Hamaker constant has little effect on 


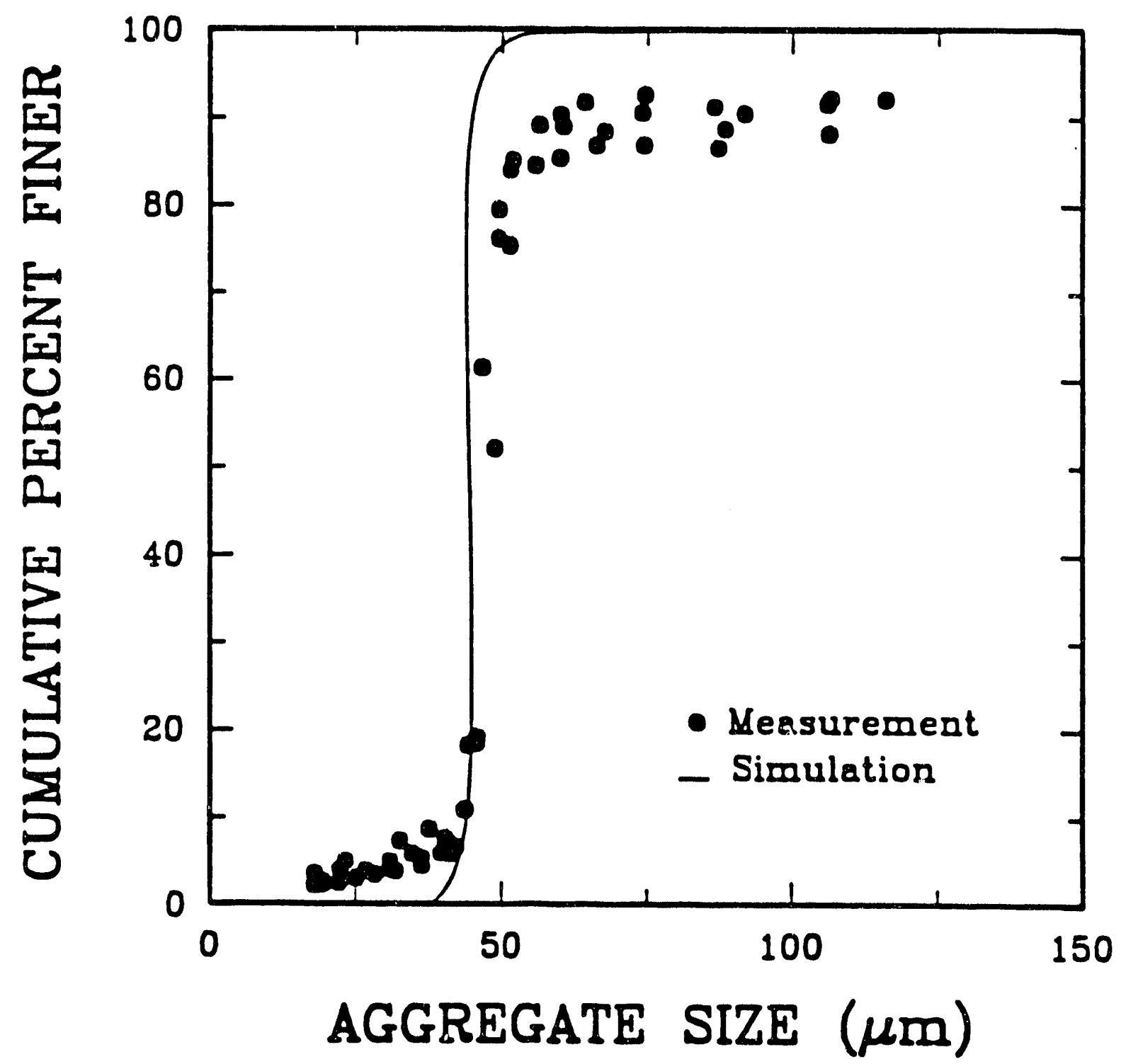

Figure 32. A comparison of aggregate size distributions obtained from computer simulation and experimental measurements at $\mathrm{pH}$ 8.4. 


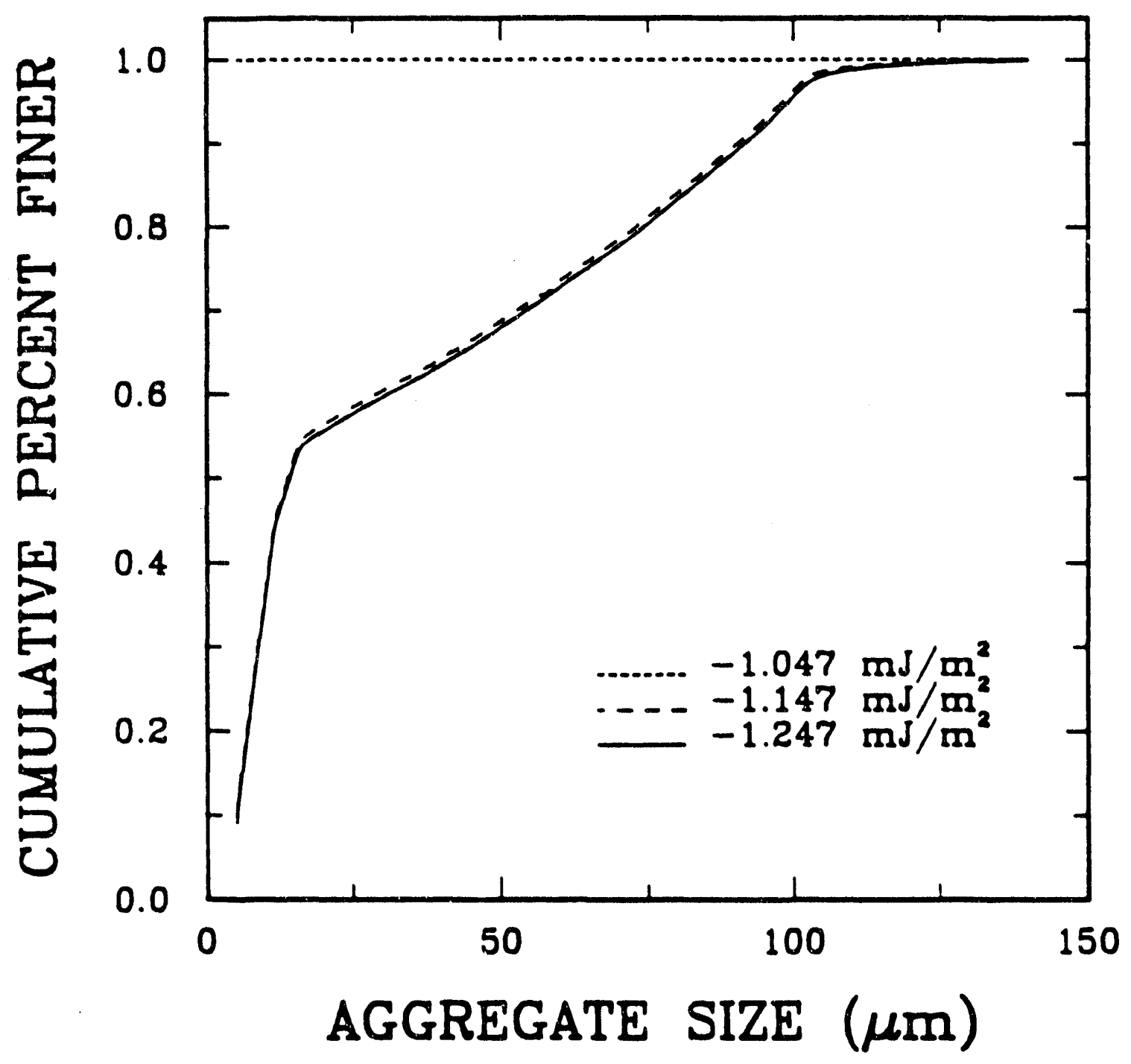

Figure 33. Equilibrium aggregate size distributions (predicted) for different values of the Hydrophobic Interaction Parameter, C. 


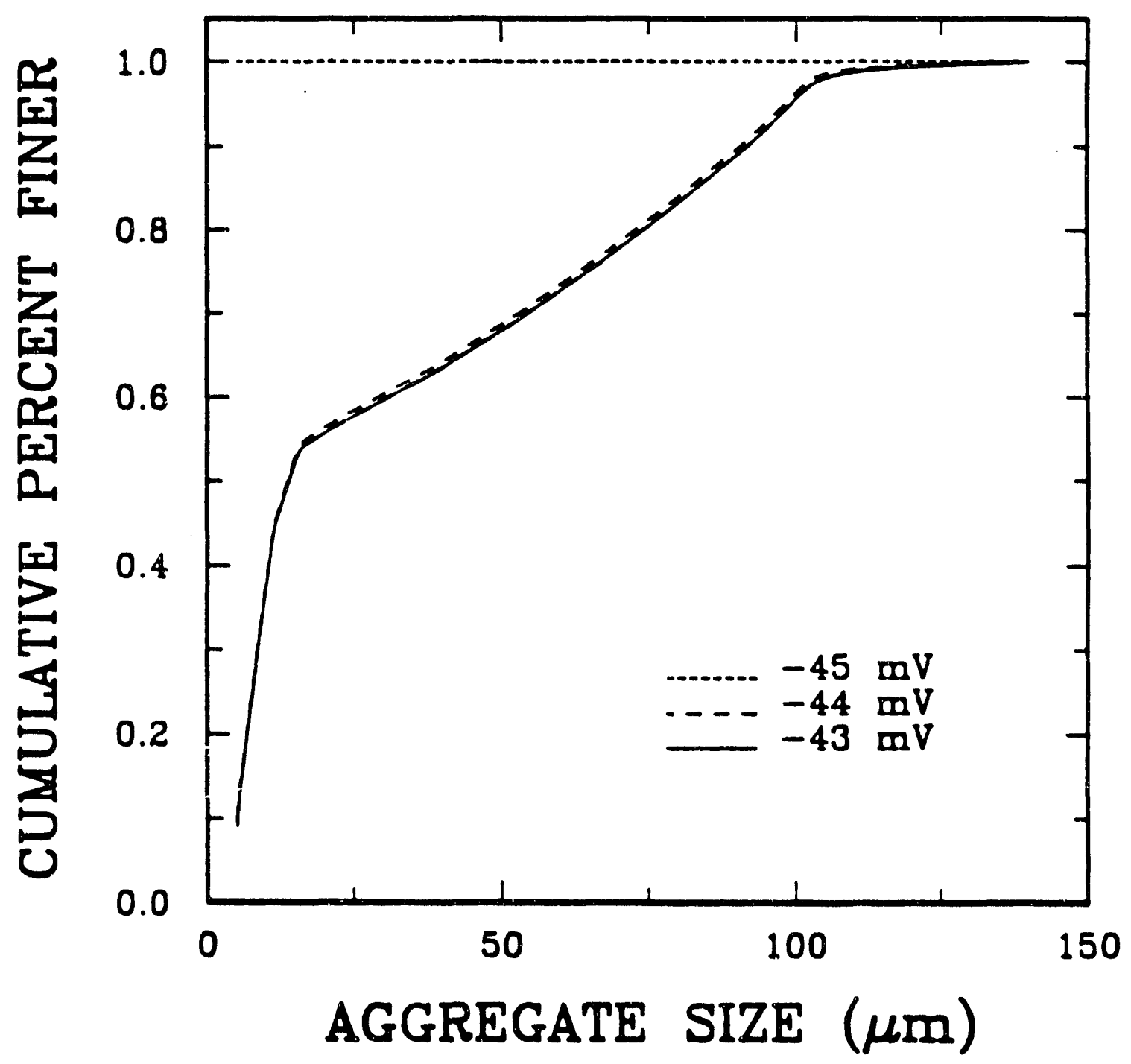

Figure 34. Equilibrium aggregate size distribution (predicted) for different values of the zeta potential. 


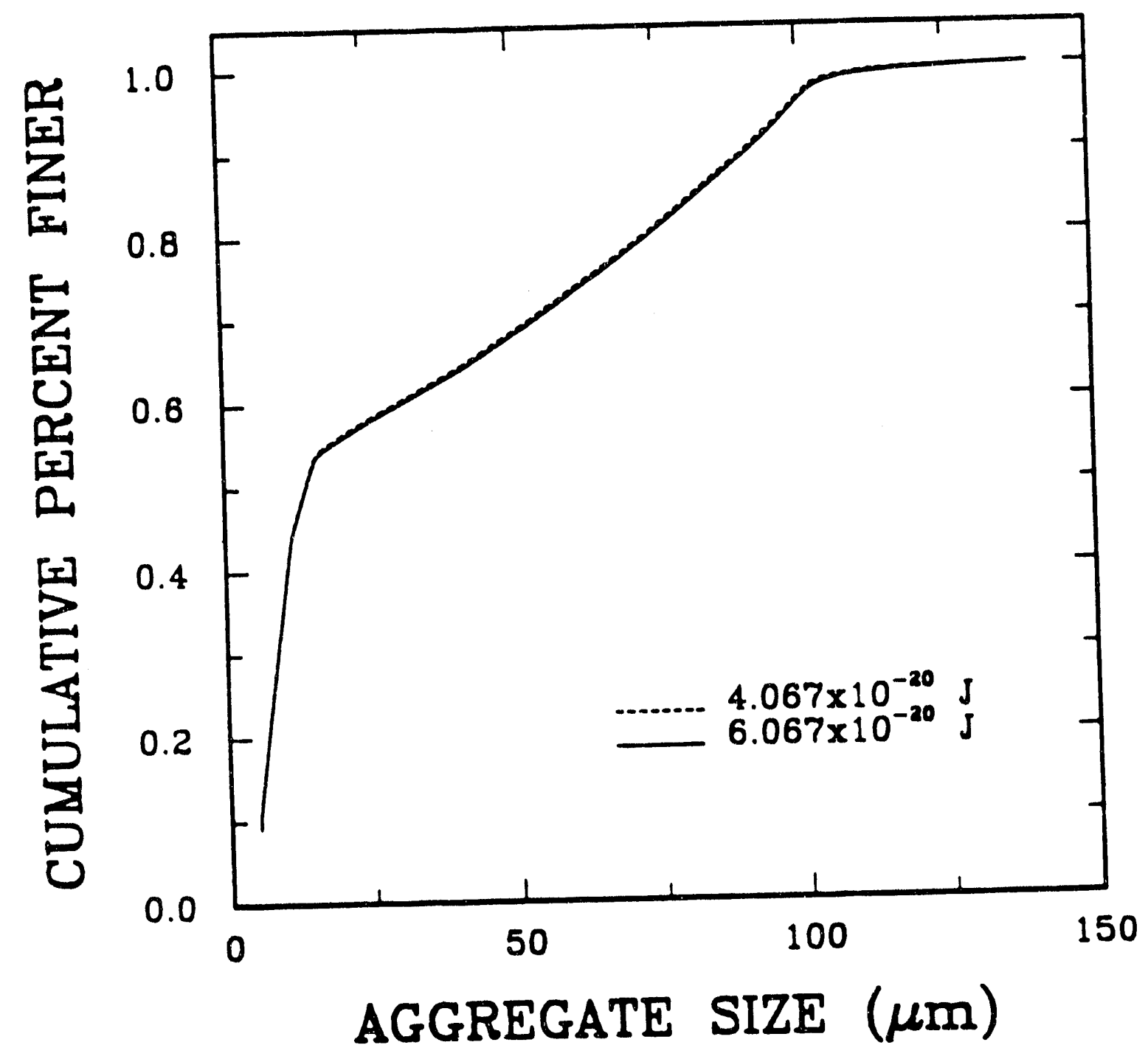

Figure 35. Equilibrium aggregate size distributions (predicted) for different values of the Hamaker constant. 
equilibrium size distribution.

Figure 36 illustrates the general effect of mixing rate on the equilibrium size distribution of aggregates. It will be noted that the topsize of the aggregates decreases as the mixing rate increases, principally because the rates of breakage of the larger aggregates begin to predominate as impeller speed increases. The model prediction for aggregate topsize as a function of agitation speed is given in Figure 37, along with experimental data generated in Task 3.3 (Mixing Studies). As shown, the degree of agreement is excellent.

\section{Task 2.4 Measurement of Coagula Size}

In order to validate the population balance model developed in Task 2.3 , a Lasentec Model 100 in-situ particle size analyzer was utilized to measure the coagula size under various mixing intensities and chemical conditions. The Lasentec particle size analyzer uses back-scattered laser light to infer the particle (aggregate) size. The width and number of the back-scatter pulses are monitored to predict the particle (aggregate) size distribution. The particle size of a solid suspension is measured by simply immersing the Lasentec probe into the particle suspension. The advantage of this technique is that the particle size analyses can be conducted in-situ.

The instrument was calibrated with samples of pre-cleaned Elkhorn No. 3 and Pittsburgh No. 8 seam coals which had been wet-screened into several size fractions between 28 and 500 mesh. First the size fractions were analyzed by an Elzone $80-X Y$ particle size analyzer to obtain the actual volume mean particle diameter $\left(D_{s 0}\right)$. Then the actual $D_{s 0}$ of each size class was compared to the corresponding $D_{s 0}$ determined using the Lasentec analyzer. As shown in Figures 38 and 39, this procedure allowed calibration 


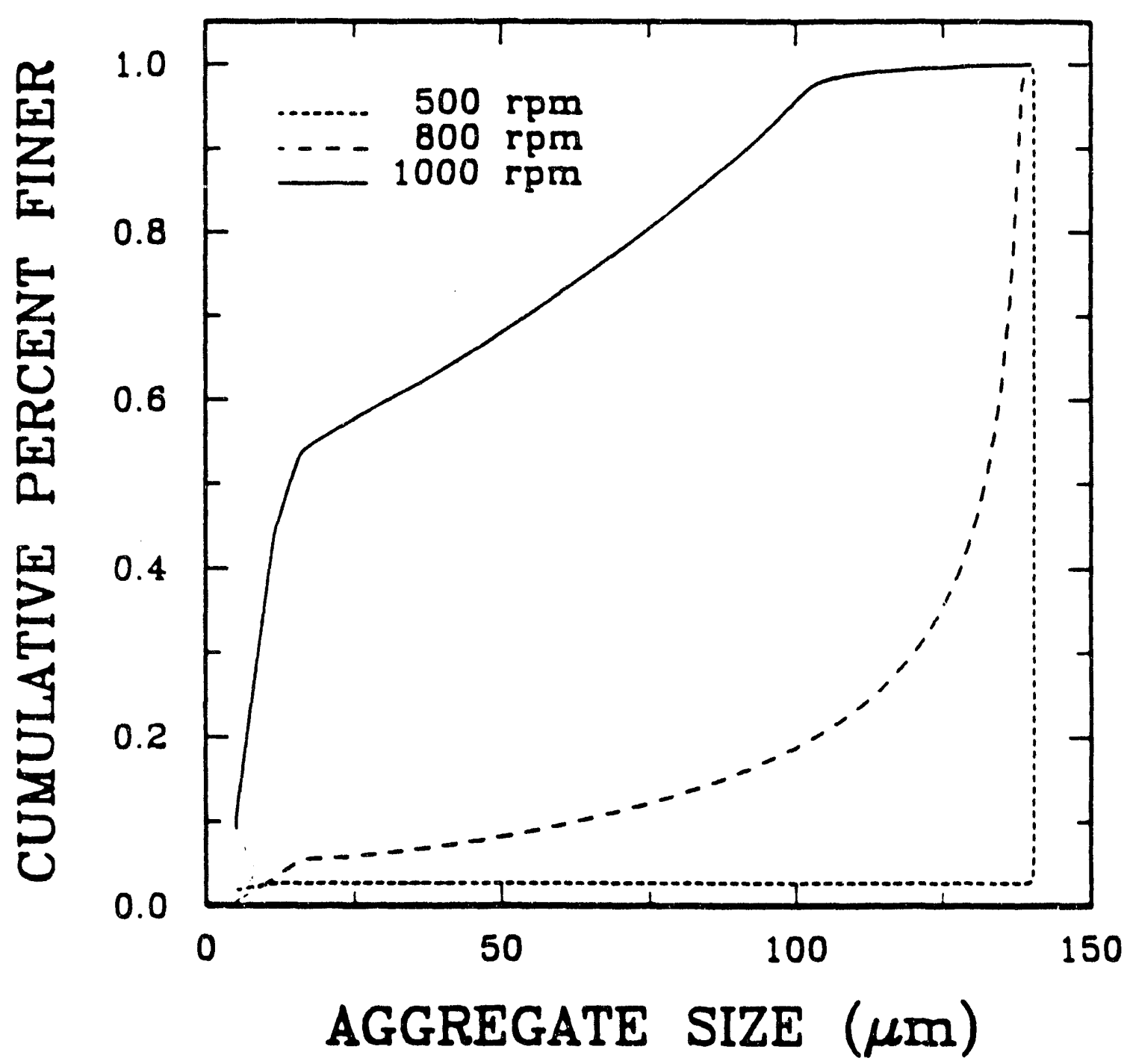

Figure 36. Equilibrium aggregate size distributions (predicted) as a function of mixing rate. 


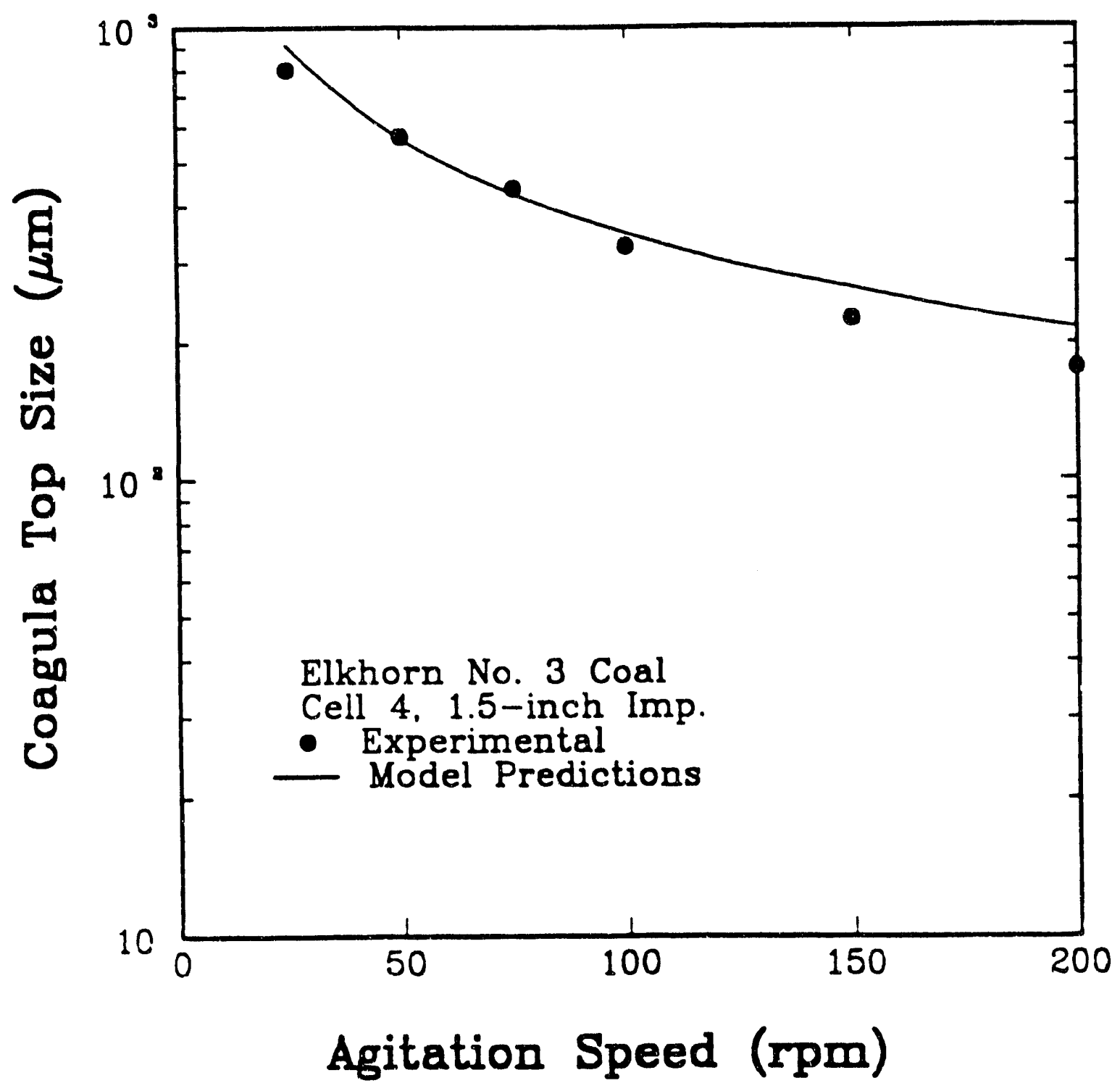

Figure 37. Coagula top size as a functlon of mixer agitator speed for the Elkhorn No. 3 coal. 


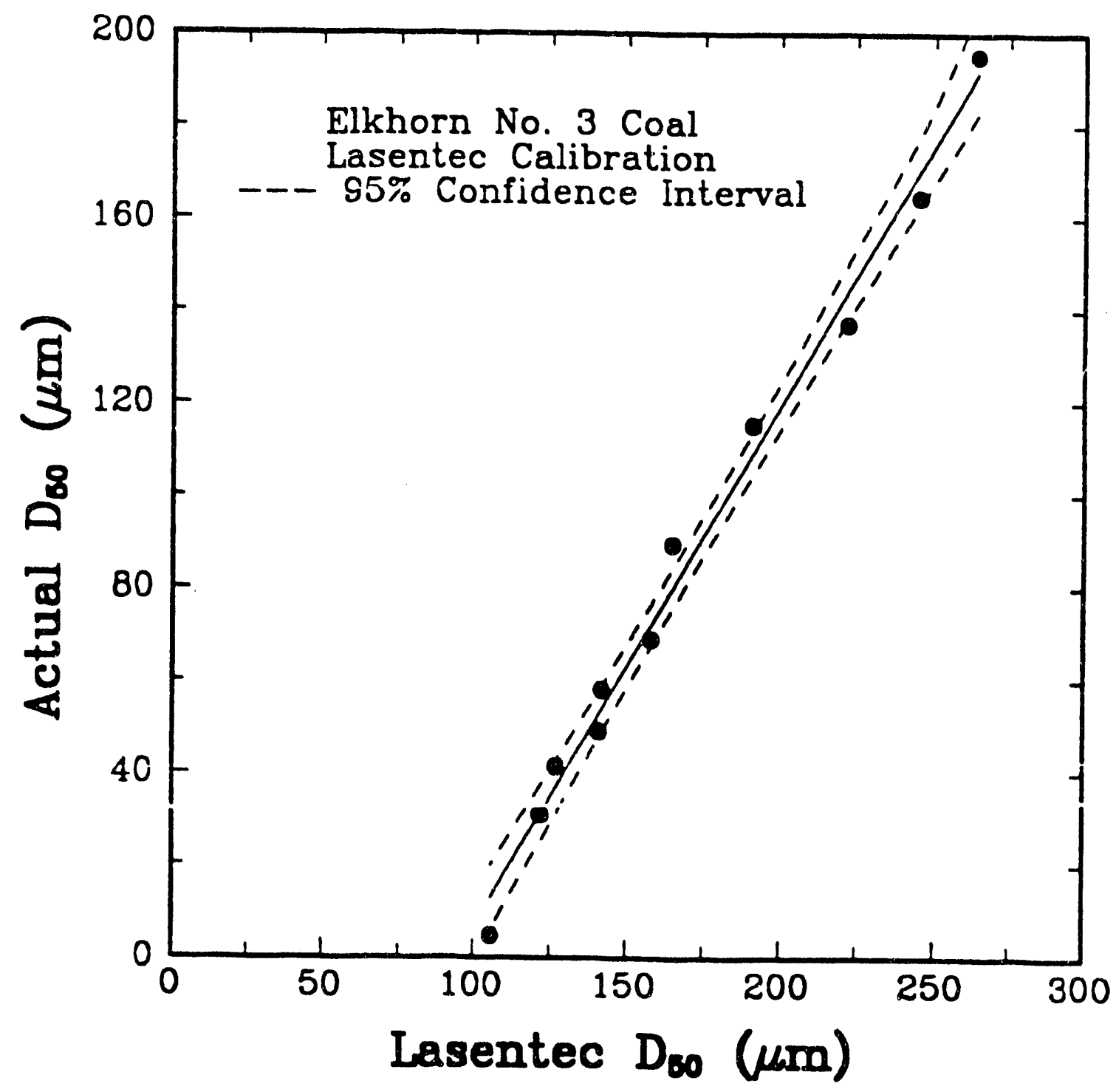

Figure 38. Actual $D_{50} v s$. Lasentec $D_{50}$ for Elkhorn No. 3 seam coal. 


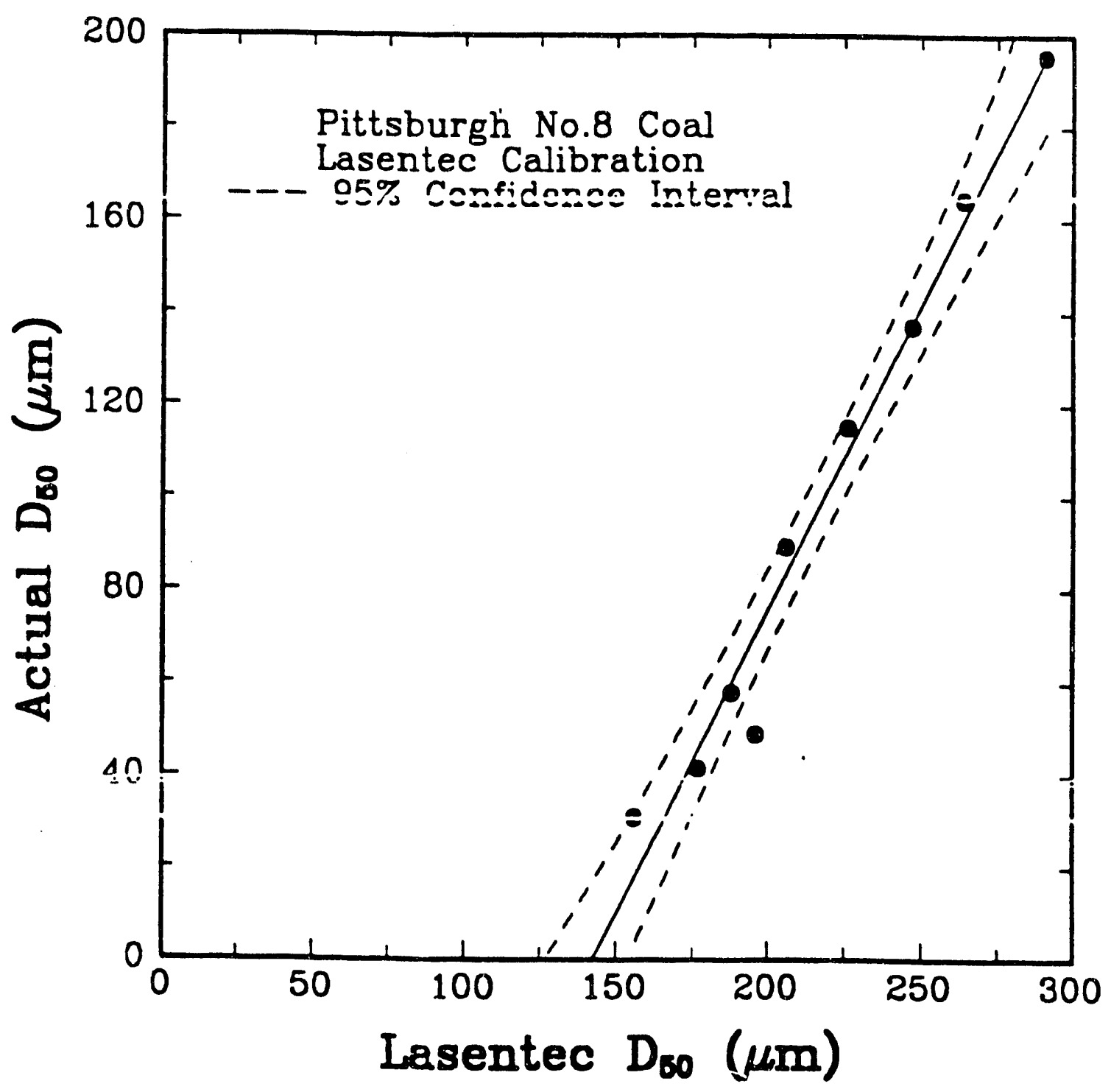

Figure 39. Actual $D_{50}$ vs. Lasentec $D_{50}$ for Pittsburgh No. 8 seam coal. 
curves to be constructed for each coal sample. The actual $D_{s o}$ for the Elkhorn No. 3 and Pittsburgh No. 8 coals could be determined from the following linear relationships:

ElkhornNo.3: Actual $D_{50}=1.13\left(\right.$ Lasentec $\left.D_{50}\right)-106.91$,

PittsburghNo.8: Actual $D_{50}=1.32$ (Lasentec $\left.D_{50}\right)-187.77$.

These equations were used to obtain actual coagula size distributions for the various test conditions. The initial size distributions obtained for dispersed and coagulated coal suspensions are shown in Figure 40. Coagulation of the coal was carried out in distilled water at a pH value of 7.5. Complete dispersion of the coal suspension was ensured by the addition of Lomar-D dispersant. Figure 40 illustrates the relatively large difference in the particle distributions of the dispersed and coagulated suspensions. The measured $D_{50}$ of the dispersed particles was found to be $4.7 \mu \mathrm{m}$ while the coagula $D_{50}$ was $290 \mu \mathrm{m}$. The $S H C$ process exploits this size difference to separate the coal coagula from the dispersed gangue material.

Since $\mathrm{pH}$ can be used to either disperse or coagulate a coal suspension, coagula size distributions were obtained for $\mathrm{pH}$ values between 7.5 and 8.5 at an agitation rate of $25 \mathrm{rpm}$. The results shown in Figure 41 indicate that the coagula size distribution decreases as the $\mathrm{pH}$ of the coal slurry is increased. By increasing the $\mathrm{pH}$ from 7.5 to 8.2 , the mean coagula size is reduced from $290 \mu \mathrm{m}$ to $88 \mu \mathrm{m}$. A further increase in $\mathrm{pH}$ to 8.5 resulted in a decrease in the $D_{s 0}$ to $45 \mu \mathrm{m}$. These results correlate well with the critical coagulation $\mathrm{pH}\left(\mathrm{pH}_{\mathrm{c}}\right)$ of 8.2 reported above for the Elkhorn No. 3 coal.

As indicated in Task 2.3 , the kinetic energy supplied by mechanical mixing can result in an increase in both the coagulation growth rate and breakage rate. The coagula size corresponding to the balance of the two rates represents the top size of the coagula size distribution. In order to characterize the effect of inechanical agitation, coagula size 


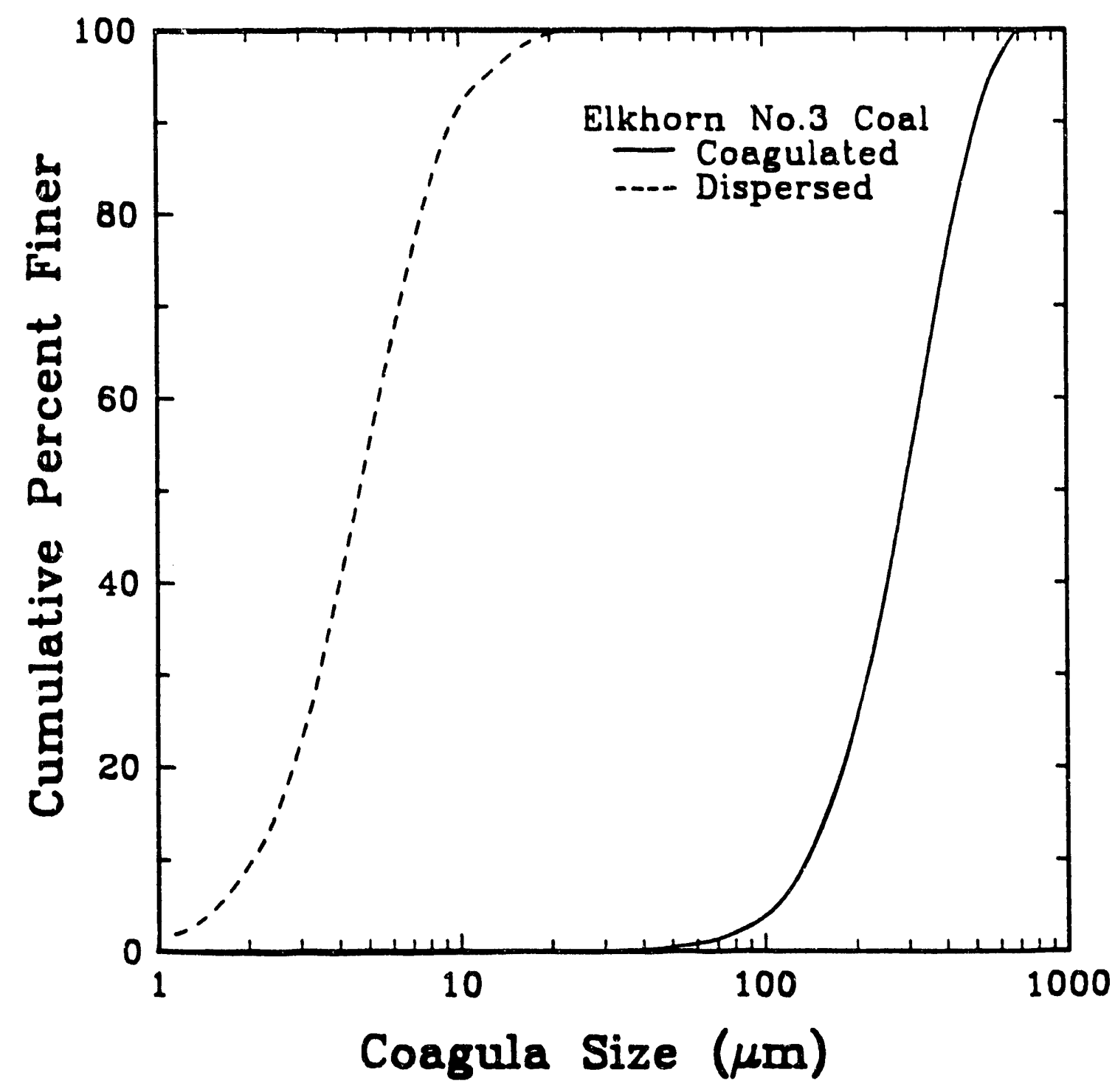

Figure 40. Coagula size distributions for the Elkhorn No. 3 seam coal measured under coagulated and dispersed conditions. 


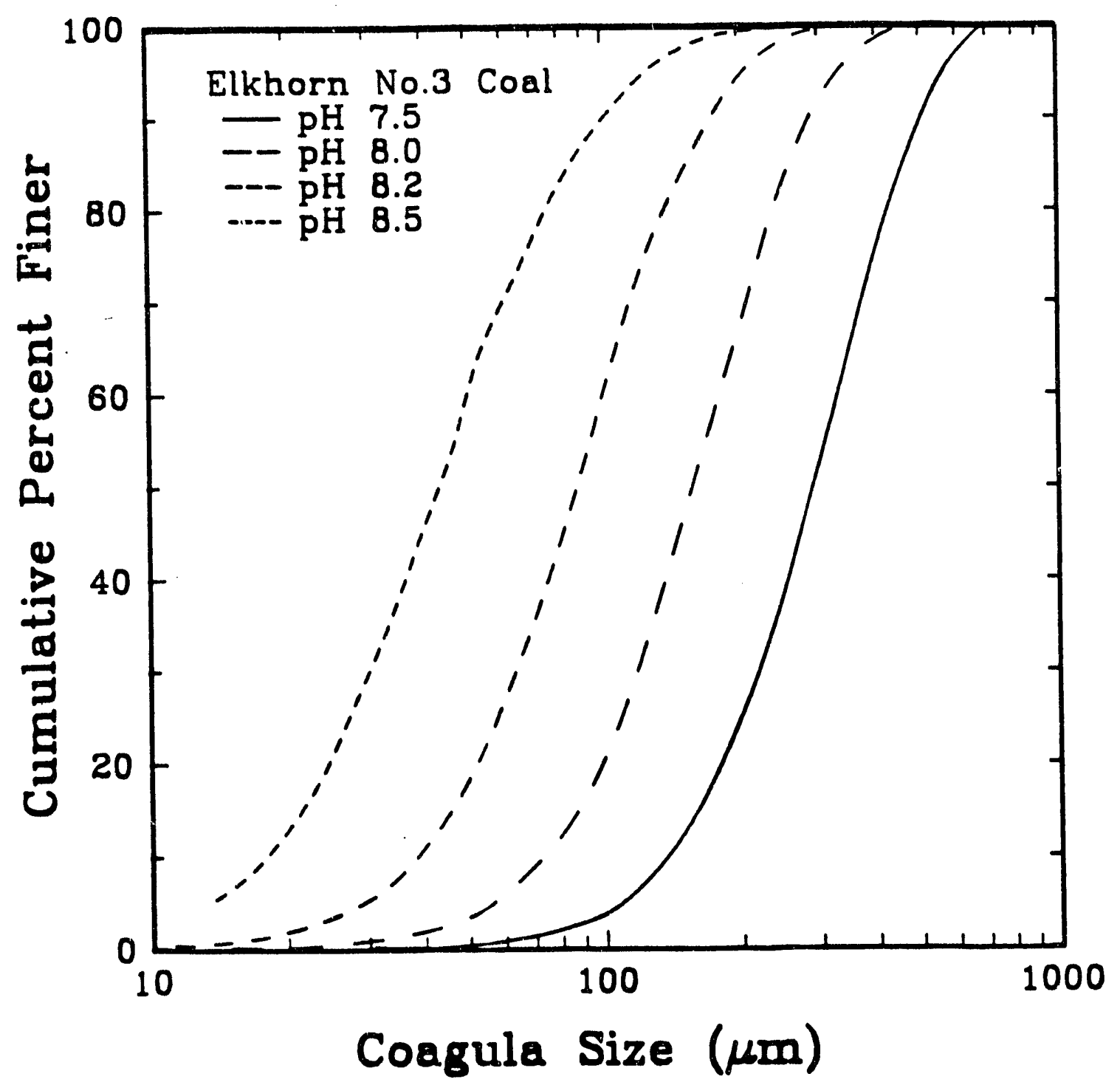

Figure 41. Effect of $\mathrm{pH}$ on the coagula size distribution for the Elkhom No. 3 seam coal. 
distributions were measured as a function of agitation speed. The results of these measurements are shown in Figure 42. As expected, an increase in agitation speed reduces the coagula size, indicating that mechanical agitation enhances the breakage rate more than the growth rate for the particulate system in this study.

By combining the results in Figures 41 and 42 , the coagula $D_{s o}$ can be plotted as a function of $\mathrm{pH}$ for agitation speeds of 25,50 , and $100 \mathrm{rpm}$. As shown in Figure 43, a $\mathrm{pH}$ window consisting of values between approximately 3.5 and 8.5 exists for each agitation rate. The maximum $D_{s 0}$ within the window was $290 \mu \mathrm{m}$ for an agitation speed of $25 \mathrm{rpm}$ compared to $160 \mu \mathrm{m}$ and $60 \mu \mathrm{m}$ for $50 \mathrm{rpm}$ and $100 \mathrm{rpm}$, respectively. This result further demonstrates the effect of mechanical agitation on coagula breakage. The upper and lower critical $\mathrm{pH}$ values correspond with $\zeta$-potentials that result in the repulsive electrostatic interaction energy exceeding the attractive interaction energies. This phenomenon can be described by the previously discussed extended DLVO theory.

Coagula size distributions for the Elkhorn No. 3 coal were measured using the Lasentec size analyzer for three different initial particle sizes and four different solids concentrations. In most tests, the effects of $\mathrm{pH}$ and impeller speed were also examined. The samples used for this study were prepared by wet-grinding in a laboratory stirred-ball mill for a predetermined amount of time to achieve the desired particle size. Following grinding, each sample was transferred to a 4-inch diameter mixing vessel having eight $1 / 4$ inch baffles, the solids concentration was adjusted to $2 \%$ solids by weight, and the $\mathrm{pH}$ was adjusted to the desired value using sodium hydroxide. Also, unless otherwise stated, potassium chloride was added as a supporting elertrolyte at a concentration of $10^{-3} \mathrm{M}$. The slurry was then agitated at $500 \mathrm{rpm}$ using a 11/2-inch diameter turbine impeller. After conditioning, the coagula size distributions were measured in-situ using the Lasentec particle size analyzer. These measurements were then corrected, using Eq. 


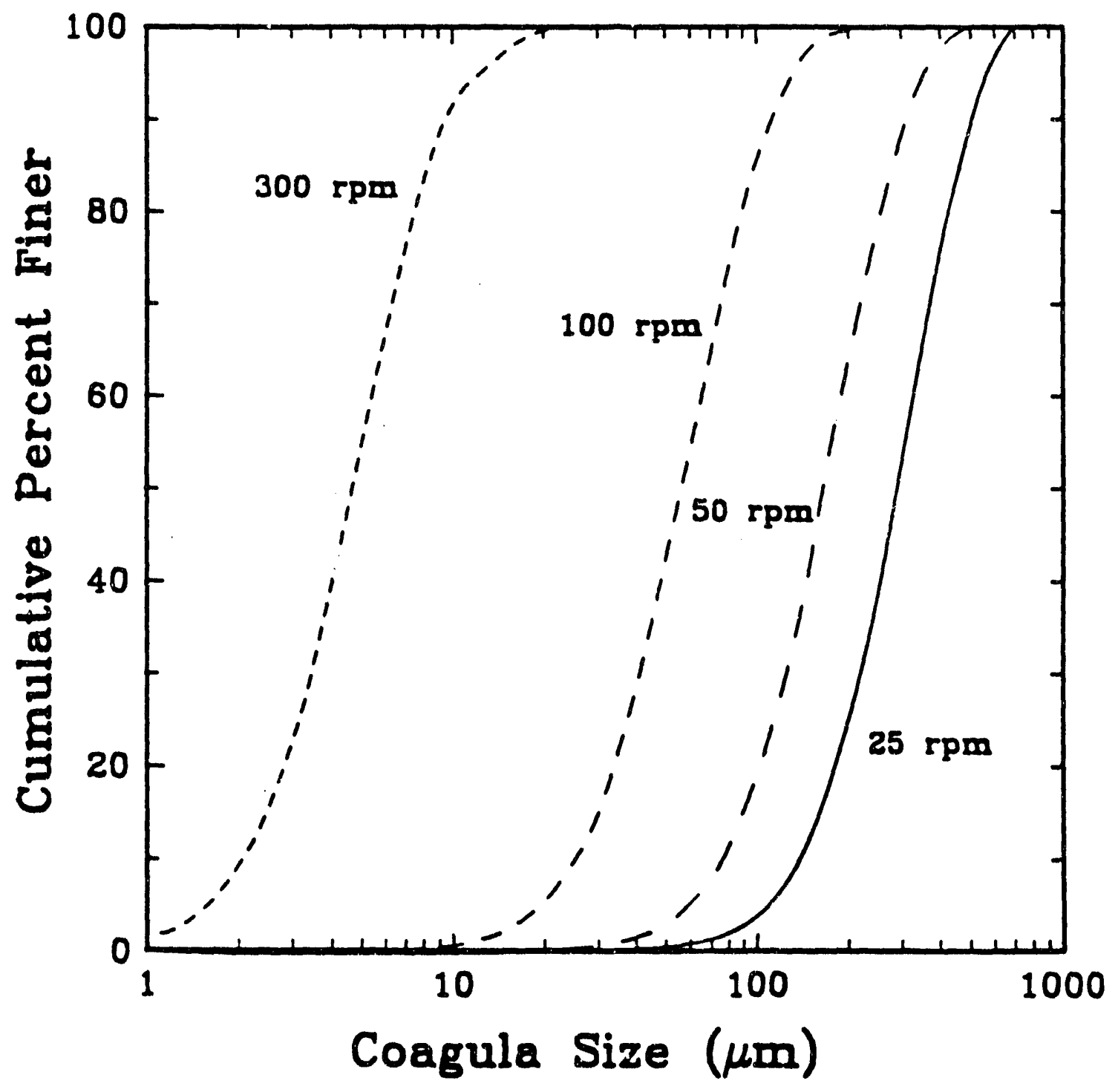

Figure 42. Effect of mixing intensity on the coagula size distribution for the Elkhorn No. 3 seam coal. 


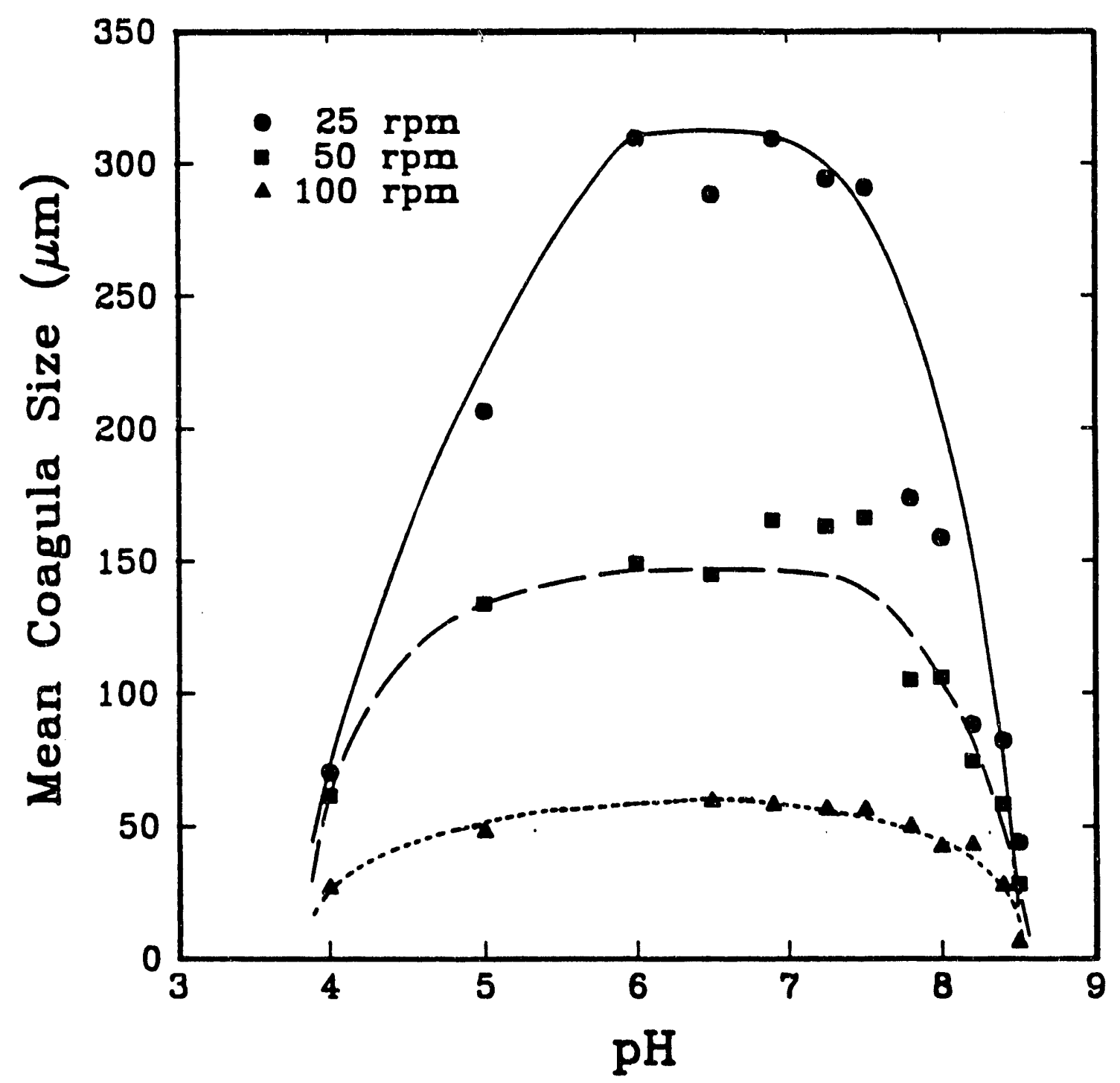

Figure 43. Effect of $\mathrm{pH}$ and mixing intensity on the mean coagula size for the Elkhorn No. 3 seam coal. 
[54], to obtain the actual $D_{50}$ of the coagula.

The effect of the initial mean particle size (IPS) on the resultant mean coagula size $\left(D_{50}\right)$ was studied as a function of agitation speed and $\mathrm{pH}$. Three different initial particle sizes were prepared by wei grinding samples of Elkhorn No. 3 coal for 2, 5, and 10 minutes in a laboratory attrition mill. Mean particie sizes for these samples were determined with an Elzone 80-XY particle size analyzer to be $7.2,5.3$ and $3.6 \mu \mathrm{m}$, respectively.

Table III. Results illustrating the effect of initial particle size on the mean coagula size over a range of $\mathrm{pH}$ values and agitation speeds.

\begin{tabular}{|c|c|c|c|c|c|c|}
\hline \multirow{2}{*}{ RPM } & \multicolumn{6}{|c|}{ Mean Coagula Size $(\mu \mathrm{m})$} \\
\cline { 2 - 7 } & \multicolumn{2}{|c|}{ At pH 7.5 } & \multicolumn{2}{c|}{ At pH 8.0 } & \multicolumn{2}{c|}{ At pH 8.5} \\
\cline { 2 - 7 } & IPS =3.6 & IPS $=7.2$ & IPS =3.6 & IPS =7.2 & IPS=3.6 & IPS $=7.2$ \\
\hline 25 & 355 & 288 & 189 & 175 & 92.1 & 50.2 \\
\hline 50 & 167 & 199 & 132 & 116 & 74.0 & 38.9 \\
\hline 75 & 90.7 & 156 & 72.8 & 48.0 & 68.3 & 31.0 \\
\hline 100 & 67.2 & 71.7 & 52.5 & 32.1 & 59.3 & 17.4 \\
\hline
\end{tabular}

IPS = Initial Particle Size (in $\mu \mathrm{m})$

Table III provides a summary of the coagula size measurements conducted as functions of initial particle size, agitation speed and pH. Only two initial particle sizes, 3.6 and $7.2 \mu \mathrm{m}$, were examined in detail in this first series of tests. For most experimental conditions, the test data indicate that the mean coagula size increases with a decrease in the initial particle size, i.e., finer feeds produce larger coagula. This finding is reasonable since (i) the particle population, and hence collision frequency, increases as the initial particle size decreases and (ii) particle detachment is reduced by a decrease in 
particle size due to the reduced magnitude of the inertial forces on finer particles. As shown in Table III, the increase in coagula size with a decreasing initial particle size is most pronounced ai pH values 8.0 and 8.5. However, when the coagulation experiments were conducted at pH 7.5, the reverse trend was observed for agitation speeds greater than $25 \mathrm{rpm}$. This behavior is illustrated more clearly in Figure 44, which shows coagula size distributions measured at $37 \mathrm{rpm}$ and $\mathrm{pH}$ 7.5. As shown, the coagula size distributions shift toward finer sizes as the initial particle size decreases.

Additional coagula size measurements were conducted at solids concentrations of $0.1 \%, 0.5 \%, 1.0 \%$, and $2.0 \%$ by weight in order to develop a better understanding of the effects of particle population on ccagulation behavior. These tests were conducted using a pre-cleaned Elkhorn No. 3 coal sample that had been ground for 5 minutes in a laboratory attrition mill. Both the pH and agitation speed were held constant, at 7.5 and $25 \mathrm{rpm}$, respectively, during this series of tests. As shown in Figure 45, there was a slight decrease in the coagula $D_{s 0}$ size from $325 \mu \mathrm{m}$ to $261 \mu \mathrm{m}$ when the sclids content was decreased from $2.0 \%$ to $1.0 \%$. Further decreases in solids content to $0.5 \%$ and $0.1 \%$ resulted in sharp drops in the coagula $D_{50}$ sizes to $104 \mu \mathrm{m}$ and $37 \mu \mathrm{m}$, respectively. These results may be explained by the fact that the number of particles per unit volume decreases as the solids content is decreased. As a result, the particle collision frequency decreases with decreasing particle population, resulting in a decrease in coagula size.

Coagula size distributions for the Pittsturgh No. 8 coal seam sample were also determined during this reporting period. In general, the coagula obtained with this coal are smaller than those obtained with the Elkhorn No. 3 coal sample. This difference may be caused by the lower characteristic hydrophobicity of the Pittsburgh No. 8 coal, as reported in Task 2.2. Typical coagula and initial size distributions, measured in-situ before and after the addition of Lomar-D dispersant to a coagulated suspension, are 


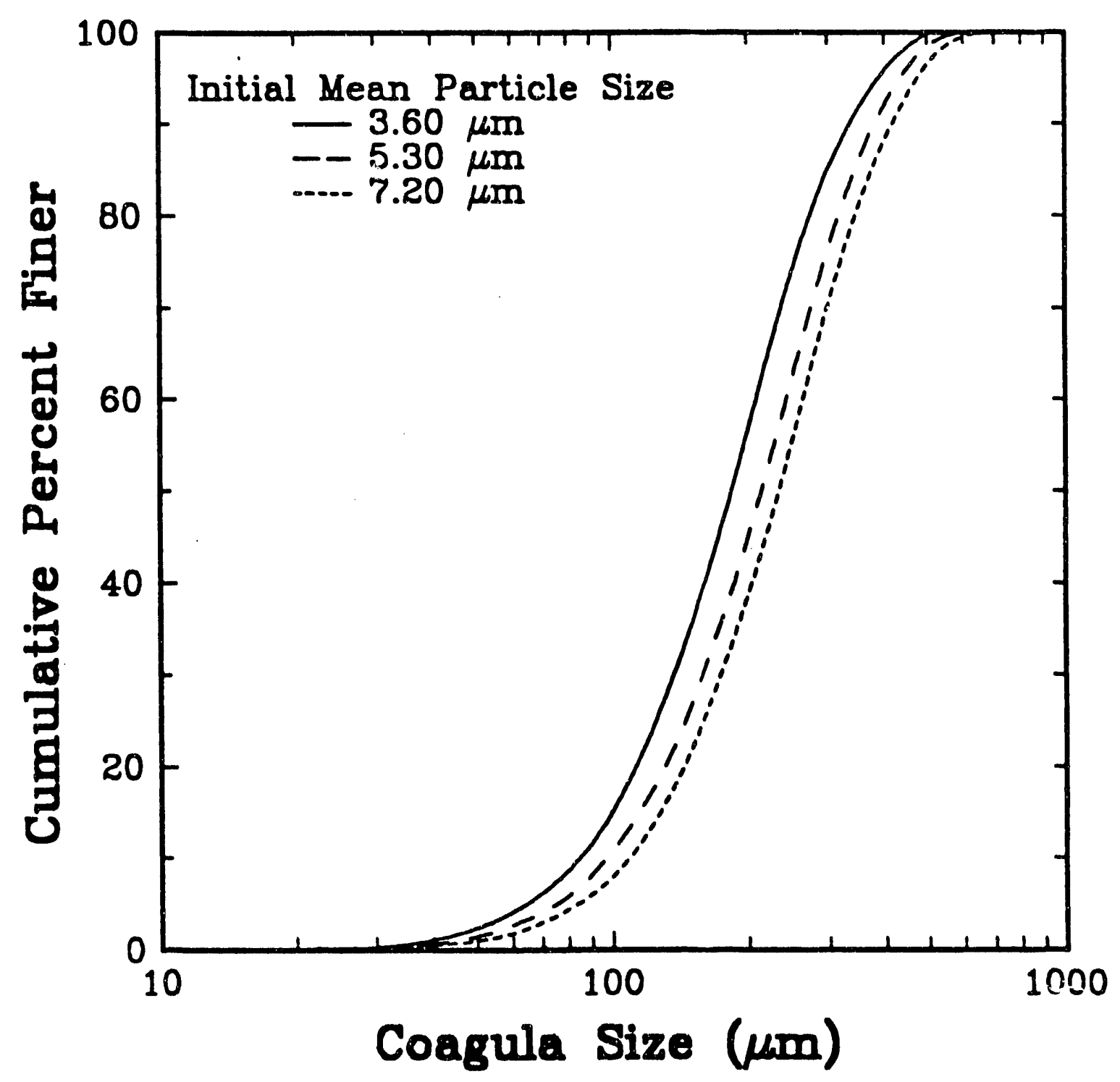

Figure 44. The effect of initial particle size on the size of coagula produced at an agitation speed of $37 \mathrm{rm}$ and $\mathrm{a} \mathrm{pH}$ of 7.5 . 


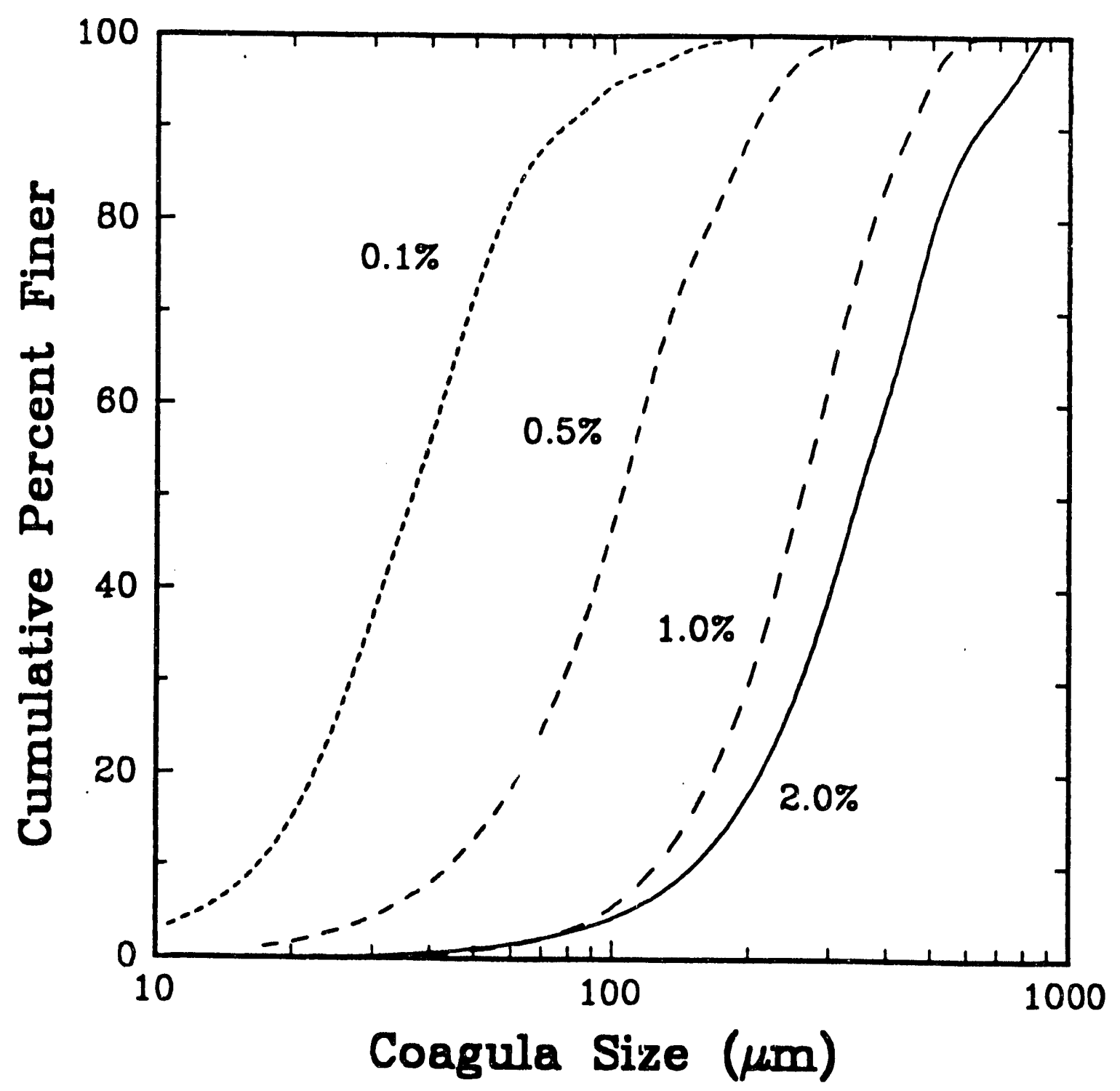

Figure 45. The effect of solids concentration on the size of coagula produced at an agitation speed of $25 \mathrm{rpm}$ and a pH of 7.5. 
compared in Figure 46. In this case, the $D_{s o}$ size of the dispersed particles was found to be $4.6 \mu \mathrm{m}$ at a $\mathrm{pH}$ value of 9 . The $D_{50}$ of the coagula was found to be $143 \mu \mathrm{m}$ at an agitation speed of $25 \mathrm{rpm}$ and $\mathrm{pH}$ of 6.5 .

In order to determine the effects of $\mathrm{pH}$ on the coagulation behavior of the Pittsburgh No. 8 seam coal, coagula size distributions were determined at pH values between 4 and 8. As shown in Figure 47, the coagula size increased as the pH was raised from 4 to 6 , and then decreased as $\mathrm{pH}$ was further increased to $\mathrm{pH} 7$. The decrease in the coagula size beycnd $\mathrm{pH} 6$ correlate well with the critical coagulation $\mathrm{pH}$ $\left(p H_{c}\right)$ of 6.2 reported above.

The effect of agitation speed on the coagulation behavior of the Pittsburgh No. 8 coal was also investigated. The coagula size distributions were measured for agitation speeds of $25,50, \mathrm{i} 00$, and $300 \mathrm{rpm}$ at a constant $\mathrm{pH}$ of 6.5 . The results of these tests, plotted in Figure 48, indicate that an increase in agitation speed causes a corresponding decrease in the coagula size distribution. The maximum mean coagula diameter of 143 $\mu \mathrm{m}$ was obtained at an agitation speed of $25 \mathrm{rpm}$, while a speed of $100 \mathrm{rpm}$ resulted in a coagula $D_{s 0}$ of less than $18 \mu \mathrm{m}$. As discussed earlier, an increase in agitation speed creates turbulence that tears the coagula apart. This indicates that the forces that hold the particles together are relatively weak, possibly because of the relatively small contact area between individual coal particles.

The combined effects of $\mathrm{pH}$ and agitation speed on the coagulation behavior of the Pittsburgh No. 8 seam coal are demonstrated in Figure 49. As shown, the optimum coagula size for the Pittsburgh No. 8 sample was obtained at the lowest agitation speed of $25 \mathrm{rpm}$ and at a pH between 6 and 6.5. It is likely that the coagula size decreased sharply above pH 6.5 due to the increased magnitude of the repulsive electrostatic interaction energy $\left(V_{R}\right)$. Calculations indicate that $V_{R}$ exceeds the attractive surface 


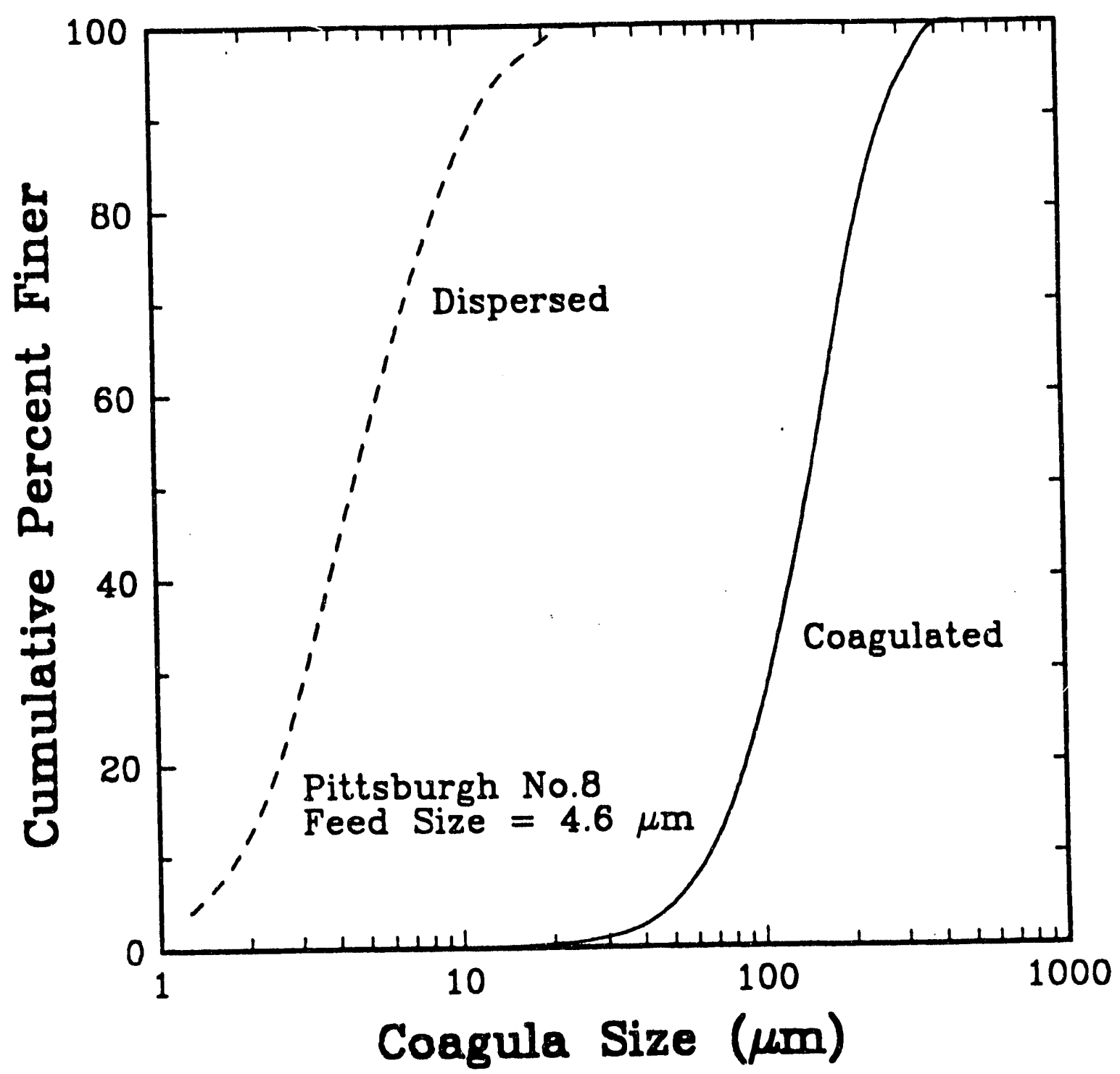

Figure 46. Comparison of the dispersed particle and coagulated particle distributions for a Pittsburgh No. 8 seam coal. 


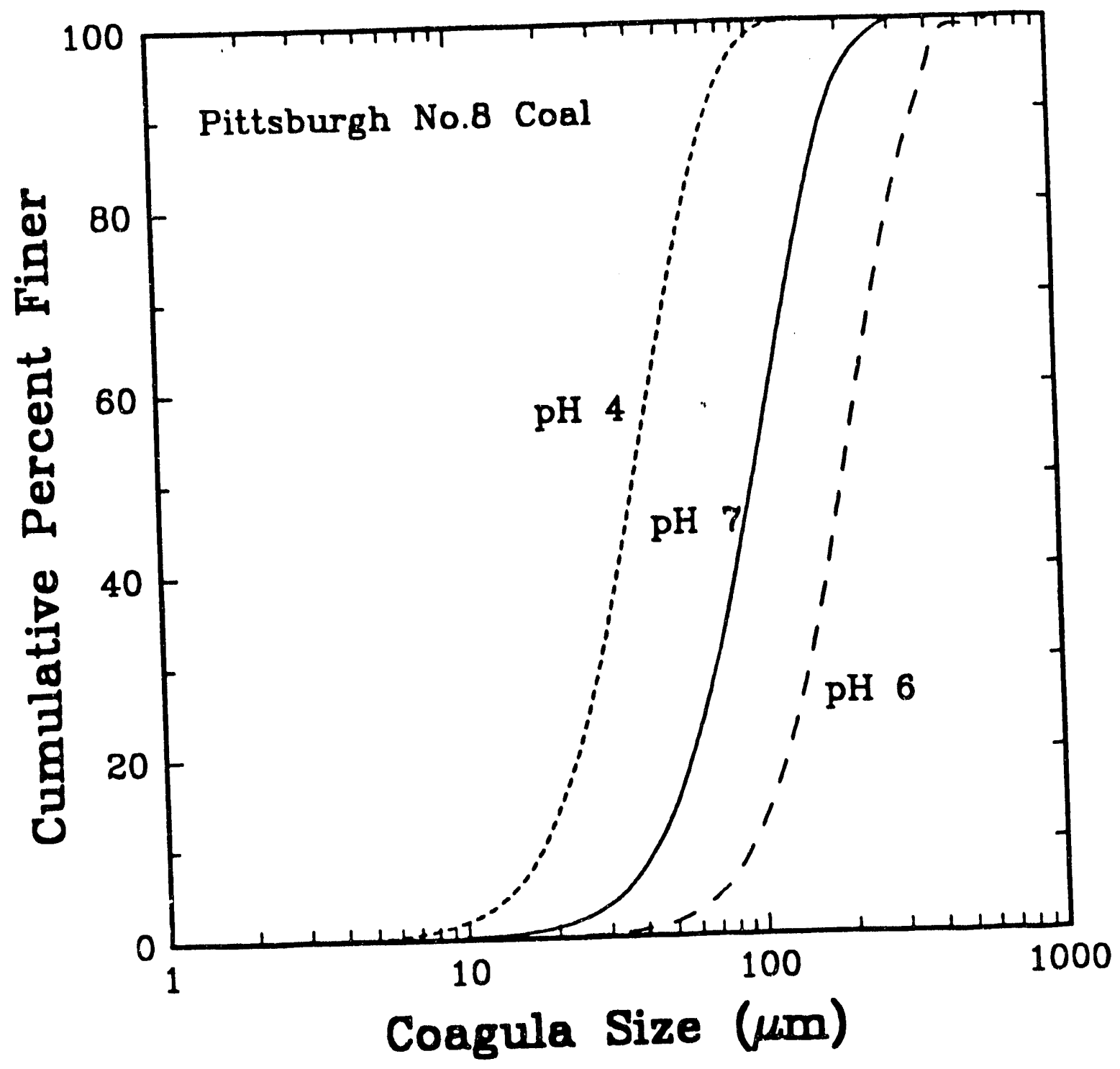

Figure 47. The effect of $\mathrm{pH}$ on the coagulation behavior of a Pittsburgh No. 8 seam coal. 


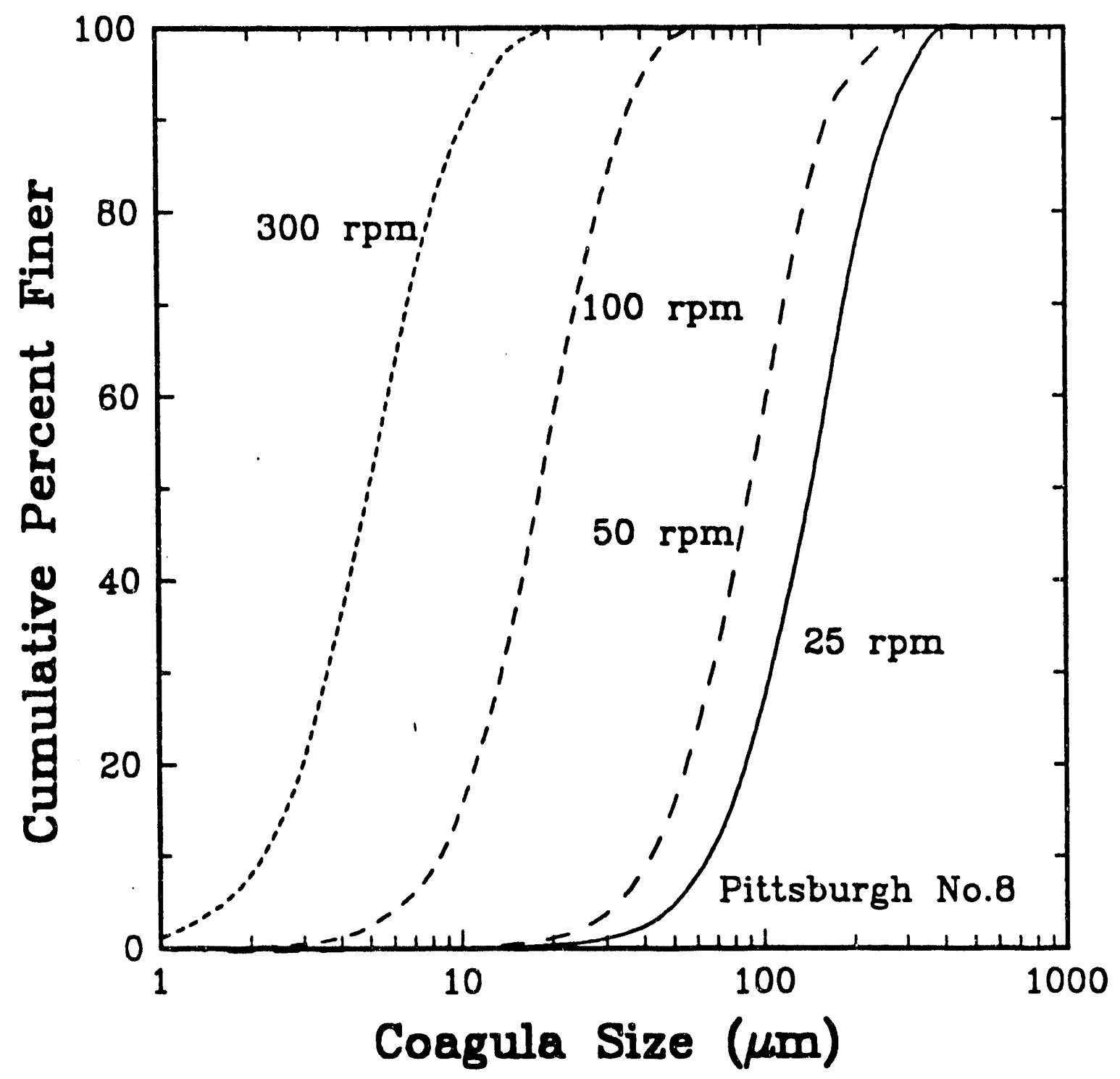

Figure 48. The effect of agitation on the coagulation behavior of a Pittsburgh No. 8 seam coal. 


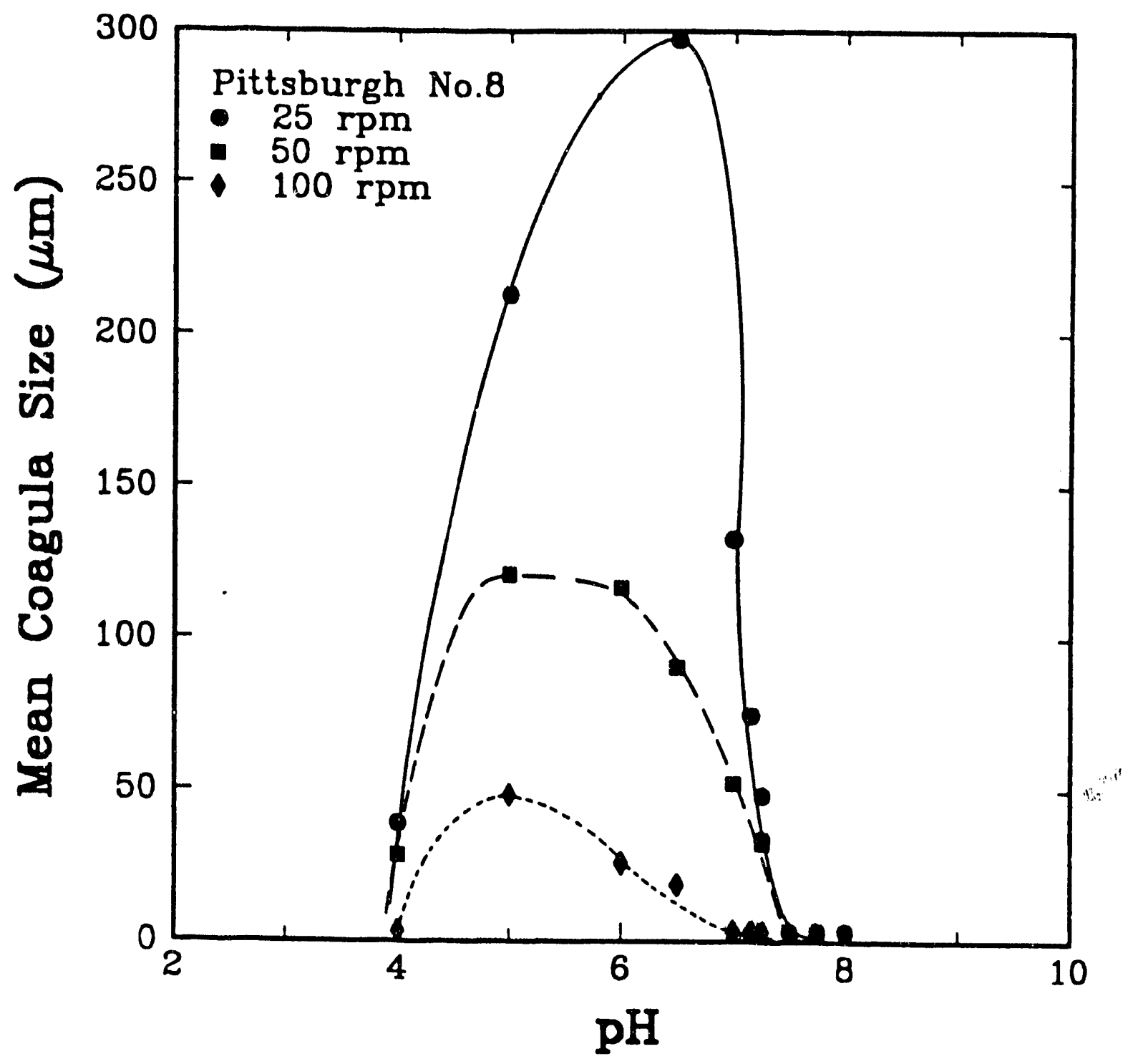

Figure 49. Mean coagula size at different mixing intensities vs. $\mathrm{pH}$ for a Pittsburgh No. 8 seam coal. 
interaction energies $\left(V_{A}\right.$ and $\left.V_{H}\right)$ above $\mathrm{pH}$ 6.2. Since the iso-electric point (iep) of the Pittsburgh No. 8 coal was found to be approximately 4.0 , the decrease in the coagula size below pH 6 is more difficult to explain.

Test work to determine the effect of hydrocarbon additions on the size of coagula obtained from Pittsburgh No. 8 coal indicated that the addition of small quantities of kerosene (1-8 lbs/ton) had little effect on coagula mean size (Figure 50). Increases in coagula size were noted at higher kerosene dosage rates (10-20 lbs/ton), particularly at low mixing speeds, but these levels are approaching those fcund in conventional, heavy oil agglomeration processes. The general effects of hydrocarbon additions on coagula size are discussed in greater detail in Task 3.4, in connection with work carried out to develop methods for enhancing the separation of coagula from dispersed material. 


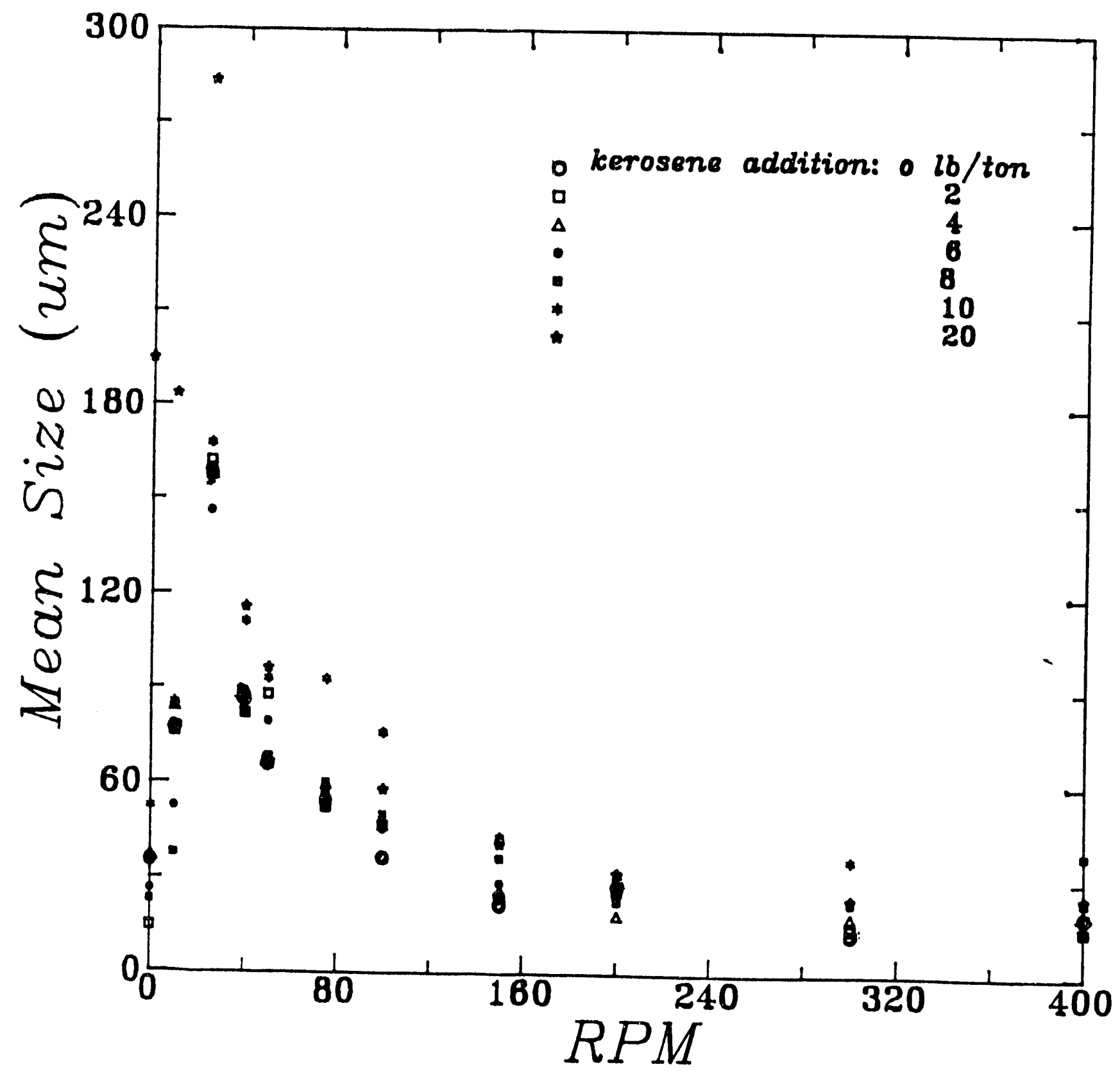

Figure 50. Effect of kerosene addition and mixing intensity on mean coagula size for Pittsburgh No. 8 coal at pH 7.0. 
TASK 3: PROCESS DEVELOPMENT

It has been shown that ultrafine hydrophobic particles can be selectively coagulated and separated from dispersed hydrophilic particles using simple $\mathrm{pH}$ control (Chapter 4). This process, referred to as selective hydrophobic coagulation (SHC), is based on the existence of a strongly attractive hydrophobic interaction energy that allows hydrophobic particles to coagulate at $\zeta$-potential values significantly larger than those predicted by the classical DLVO theory. As a result, separation can be achieved without the use of agglomerants, flocculating agents, or dispersants. Batch tests have proven that the $S H C$ process has the ability to produce superclean (less than $2 \%$ ash, less than $1 \%$ sulfur) coal while maintaining a high recovery of the valuable material.

The objectives of the process development test work described in the following tasks were to develop (i) a better understanding of the factors which control the effectiveness of the SHC process and (ii) efficient techniques for separating coal agglomerants formed by hydrophobic coagulation from unwanted mineral matter that remains dispersed. To accomplish these objectives, several bench-scale studies were carried out during the effective duration of this contract. Information collected from these studies has been used to further improve the commercial viability of the SHC process for the treatment of fine and ultrafine cods.

\section{Task 3.1 Sample Acquisition}

One 55-gallon drum channel sample from the Pittsburgh No. 8 coal seam was obtained from a Consolidation Coal Company preparation plant located in Eastern Ohio. The sample was obtained as a coarse coal product from a jig. Upon arrival, the sample was ground to $-1 / 4$-inch and split into representative lots of 1000 grams each. The 
samples were then placed into air-tight bags and stored in a freezer at a temperature of $20^{\circ} \mathrm{C}$ in order to prevent oxidation.

Two 55-gallon drums of Elkhorn No. 3 coal seam were obtained as channel samples from United Fuels Company in eastern Kentucky. Upon arrival, both barrels were crushed in a laboratory roll crusher to pass approximately 1/4-inch. One barrel was split into representative lots of 1000 grams each and placed into air-tight storage bags. The other barrel of coal was cleaned in a heavy media bath having a specific gravity of 1.3. The float fraction was air-dried and placed into air-tight storage bags. All the samples were stored in a freezer at a temperature of $-20^{\circ} \mathrm{C}$ in order to retard oxidation.

\section{Task 3.2 Sample Characterization}

Representative samples of Elkhorn No. 3 and Pittsburgh No. 8 seam coals were subjected to laboratory analyses for the determination of ash, volatile matter, fixed carbon, total sulfur, sulfur forms and heating value. The results of these analyses are summarized in Table IV. As shown, the Elkhom No. 3 seam possessed a higher ash content $(22.2 \%$ versus $14.2 \%)$ and a lower total sulfur content (1.87\% versus $5.68 \%)$ than the Pittsburgh seam. Both samples contained a relatively high percentage of pyritic sulfur, i.e., $36.4 \%$ for the Pittsburgh seam and $51.3 \%$ for the Elkhorn seam.

In order to investigate the effects of grind size on the liberation behavior of these coals, each seam was wet-ground in an attrition mill for a different length of time and then analyzed using an SEM-IPS image processing system. Grinding times of 5, 10, and 30 minutes were selected for this study. The resultant size distributions are plotted in Figures 51 and 52 , and the distribution parameters for each grind are summarized in Table V. 
Table IV. Results of sulfur forms, total sulfur, proximate, and calorimetry analyses conducted on Pittsburgh No. 8 and Elkhorn No. 3 coal samples.

\begin{tabular}{|l|c|c|}
\hline & Pittsburgh No. 8 & Elkhorn No. 3 \\
\hline Ash $\%$ & 14.18 & 22.16 \\
\hline Vol. Matter $\%$ & 37.00 & 31.91 \\
\hline Fixed Carbon $\%$ & 48.82 & 45.86 \\
\hline Btu/lb & 12,653 & 11,857 \\
\hline Total Sulfur $\%$ & 5.68 & 1.87 \\
\hline Pyritic Sulfur $\%$ & 2.07 & 0.96 \\
\hline Organic Sulfur $\%$ & 3.61 & 0.91 \\
\hline
\end{tabular}

All results on dry-basis

Table V. Product size distribution parameters for attrition grinding of Pittsburgh No. 8 and Elkhorn No. 3 coal samples.

\begin{tabular}{||ll|c|c|}
\hline & & Pittsburgh No. 8 & Elkhorn No. 3 \\
\hline 5 Minute Grind: & $D_{s 0}(\mu \mathrm{m})$ & 10.64 & 9.00 \\
& $D_{s 0}(\mu \mathrm{m})$ & 5.30 & 5.42 \\
\hline \multirow{2}{*}{ 10 Minute Grind: } & $D_{s o}(\mu \mathrm{m})$ & 9.76 & 8.18 \\
& $D_{s 0}(\mu \mathrm{m})$ & 3.90 & 4.14 \\
\hline \multirow{2}{*}{30 Minute Grind: } & $D_{s o}(\mu \mathrm{m})$ & 8.80 & 4.75 \\
& $D_{s 0}(\mu \mathrm{m})$ & 3.31 & 3.03 \\
\hline
\end{tabular}

Using the SEM-IPS system, the degree of liberation of mineral matter was deternined from the back-scattered images. The results of these measurements are summarized in Tables VI and VII for the Elkhorn No. 3 and Pittsburgh No. 8 seams, respectively. The size-by-size data collected from these studies are provided in Appendix A. The test results indicate that less than $50 \%$ of either of the feed samples had a 


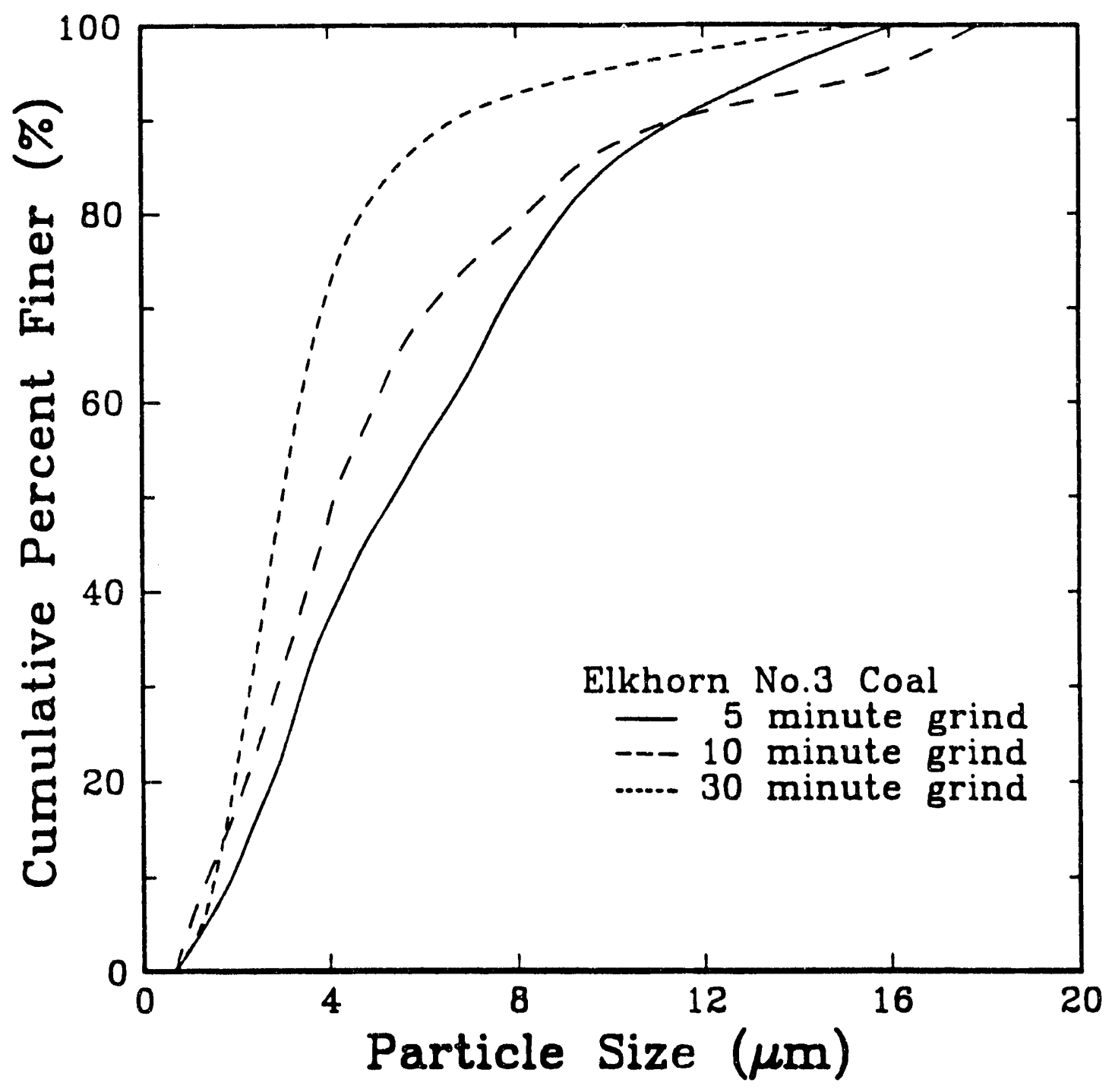

Figure 51. Effect of grind time on the particle size distribution of Elkhom No. 3 coal. 


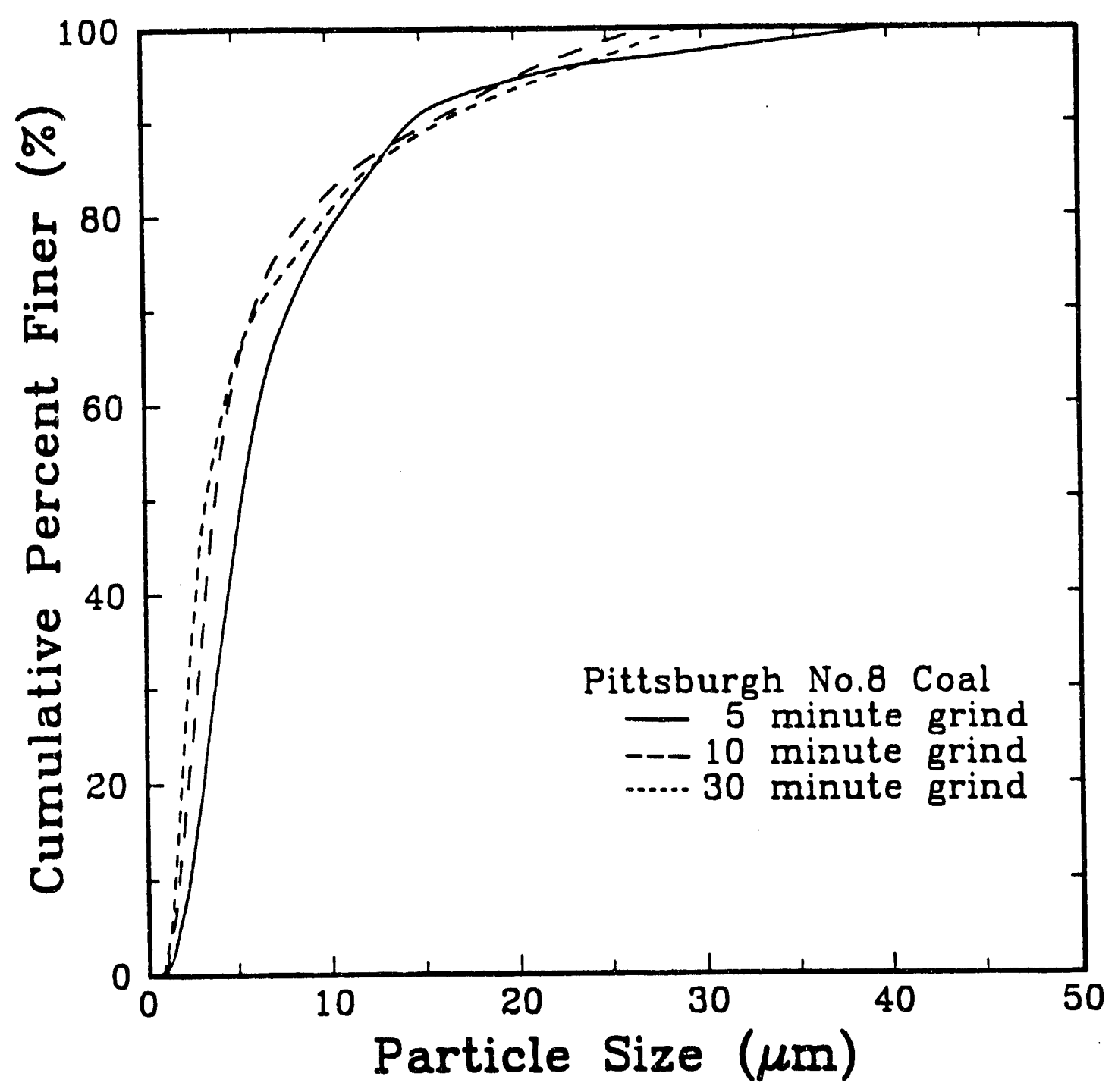

Figure 52. Effect of grind time on the particle size distribution of Pittsburgh No. 8 coal. 
mineral matter content less than $5 \%$. This finding suggests that attrition grinding would probably be necessary to produce low-ash fuels from either of these coals. For example, the amount of material having less than $5 \%$ mineral matter increased from 38.1 to $46.2 \%$ for the Elkhorn No. 3 coal sample after attrition grinding for 10 minutes. At the same time, the material containing less than $20 \%$ mineral matter increased from 54.8 to $62.6 \%$. The material containing less than $5 \%$ mineral matter material in the Pittsburgh No. 8 coal also increased from 53.2 to $58.7 \%$ after attrition grinding for 10 minutes.

Table VI. Weight distribution of mineral matter as a function of grinding time for an Elkhorn No. 3 coal seam sample.

\begin{tabular}{|c|c|c|c|}
\hline $\begin{array}{c}\text { Mineral Matter } \\
(\%)\end{array}$ & $\begin{array}{c}\text { Attrition } \\
\text { Mill Feed }\end{array}$ & $\begin{array}{c}\text { 5 Minute } \\
\text { Grind }\end{array}$ & $\begin{array}{c}\text { 10 Minute } \\
\text { Grind }\end{array}$ \\
\hline$<5$ & 38.1 & 45.6 & 46.2 \\
\hline $5-20$ & 16.7 & 18.2 & 16.4 \\
\hline $20-40$ & 8.89 & 16.3 & 10.4 \\
\hline $40-60$ & 7.79 & 10.1 & 9.99 \\
\hline $60-80$ & 10.0 & 5.31 & 9.26 \\
\hline $80-95$ & 10.8 & 3.88 & 7.77 \\
\hline$>95$ & 7.66 & 0.70 & 0 \\
\hline
\end{tabular}

Based on these results, it appears that a grinding time greater than 10 minutes may be justified in order to produce superclean coal at an acceptable combustible recovery for both the coals tested. It should also be noted that the samples ground for 30 minutes were also subjected to SEM-IPS analyses. Unfortunately, the SEM-IPS system was incapable of accurately discriminating between the various types of sub-micron (i.e., less 
Table VII. Weight distribution of mineral matter as a function of grinding time for a Pittsburgh No. 8 coal seam sample.

\begin{tabular}{|c|c|c|c|}
\hline $\begin{array}{c}\text { Mineral Matter } \\
(\%)\end{array}$ & $\begin{array}{c}\text { Attrition } \\
\text { Mill Feed }\end{array}$ & $\begin{array}{c}\text { 5 Minute } \\
\text { Grind }\end{array}$ & $\begin{array}{c}\text { 10 Minute } \\
\text { Grind }\end{array}$ \\
\hline$<5$ & 53.2 & 46.5 & 58.7 \\
\hline $5-20$ & 23.3 & 19.6 & 13.1 \\
\hline $20-40$ & 8.88 & 8.36 & 7.67 \\
\hline $40-60$ & 4.47 & 6.58 & 6.78 \\
\hline $60-80$ & 3.78 & 6.28 & 4.36 \\
\hline $80-95$ & 4.01 & 3.96 & 3.50 \\
\hline$>95$ & 2.37 & 8.79 & 5.81 \\
\hline
\end{tabular}

than $1 \mu \mathrm{m}$ ) particles contained in the finely pulverized coal products. Although several attempts were made, the extent of liberation could not be determined using the SEM-IPS system for this grind size.

To further examine the liberation characteristics of the Pittsburgh No. 8 and Elkhorn No. 3 seam coals, the particle size distributions for coal pyrite and other mineral matter were measured by subjecting briquetted samples to image analysis. The particle size distributions for the Elkhorn No. 3 coal indicate that the mineral matter, excluding pyrite, has a relatively coarse liberation size $\left(D_{50} \approx 220 \mu \mathrm{m}\right)$, whereas the coal pyrite has a finer liberation size $\left(D_{s 0} \approx 19 \mu \mathrm{m}\right)$ as shown in Figure 53. Extensive liberation of the coal pyrite and other mineral matter appears to occur at sizes finer than approximately $3.2 \mu \mathrm{n}$, which corresponds closely to the $D_{s o}$ obtained after grinding for 30 minutes.

For the Pittsburgh No: $8 \mathrm{coal}$, the coal pyrite was found to have a slightly coarser liberation size $\left(D_{s 0} \approx 380 \mu \mathrm{m}\right)$ than the other mineral matter (Figure 54). Extensive liberation of the coal pyrite seems to be achievable at approximately $10 \mu \mathrm{m}$, whereas the 


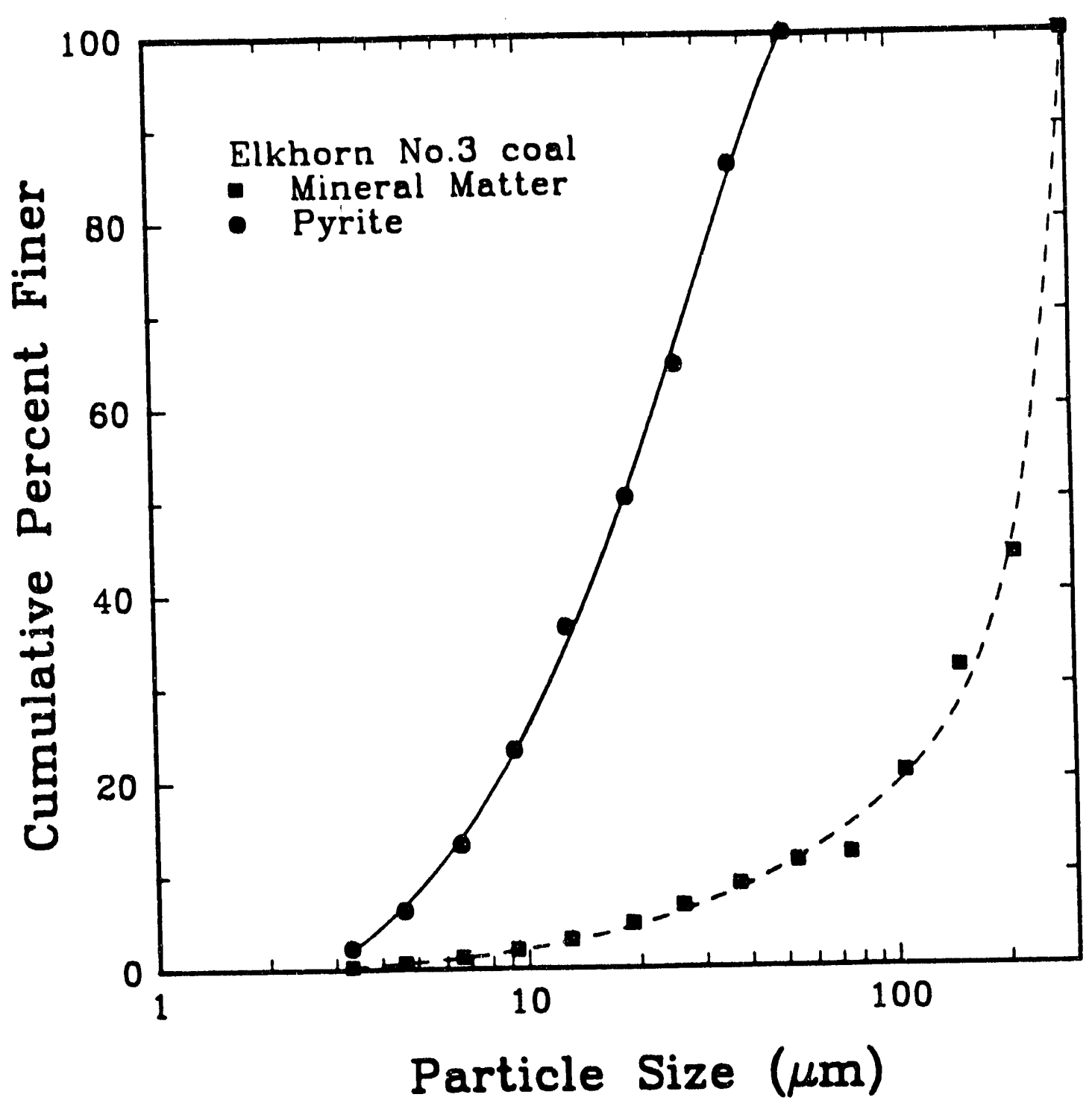

Figure 53. Grain size distributions for the Elkhorn No. 3 seam coal determined by SEM/IPS image analysis. 


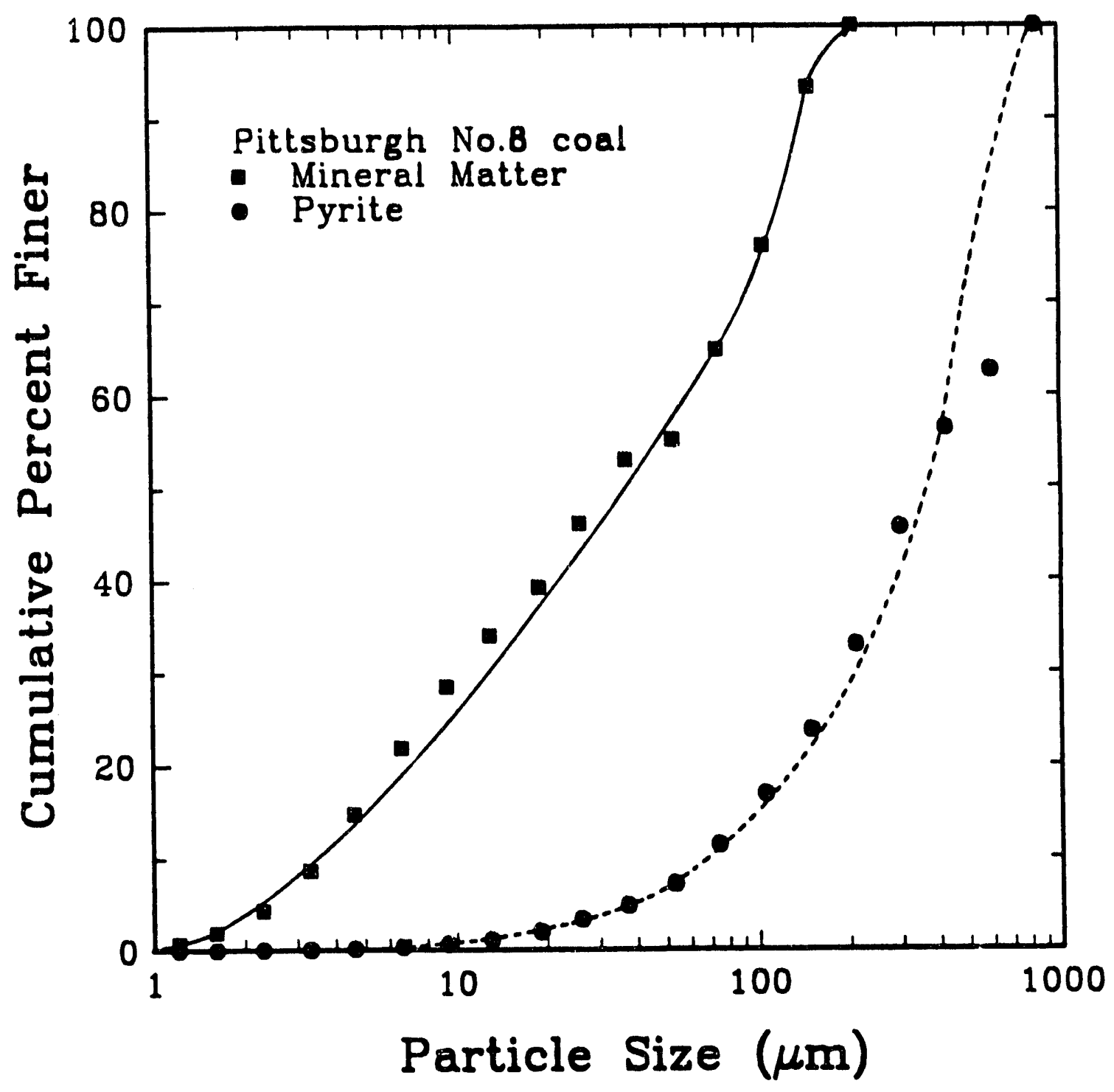

Figure 54. Grain size distributions for the Pittsburgh No. 8 seam coal determined by SEM/IPS analysis. 
mineral matter requires a particle size approaching $1 \mu \mathrm{m}$. After a 30-minute grind, the $D_{80}$ for a Pittsburgh No. 8 coal sample was measured to be $10 \mu \mathrm{m}$, which corresponds to approximately $80 \%$ liberation of the coal from pyrite. Due to the ultrafine size of the mineral matter, a cleaning scheme for the Pittsburgh No. 8 must include near complete rejection of pyritic sulfur as well as maximum possible removal of mineral matter.

For comparison sake, centrifugal washability studies were also carried out on both the Elkhorn No. 3 and Pittsburgh No. 8 seam coals. In these tests, the finely pulverized samples obtained after attrition grinding for 5, 10 and 30 minutes were passed through a Sharples Model T-1 Super Centrifuge. For cost and safety reasons, concentrated solutions of inorganic salt $\left(\mathrm{ZnCl}_{2}\right)$ were used to produce heavy-liquids having the desired specific gravities. In a typical test run, approximately $150 \mathrm{gms}$ of coal and 15 liters of salt solution (SG 1.7) were mixed together in an agitated sump. The coal/salt suspension was pumped through the centrifuge at approximately $400 \mathrm{ml} / \mathrm{min}$. A centrifuge speed of $50,000 \mathrm{rpm}( \pm 1000 \mathrm{rpm})$ was employed in all tests. The salt solution and float fractions continuously exited the centrifuge via an overflow weir, while the sink fraction was retained within the centrifuge bowl. After emptying the feed sump, several liters of fresh salt solution of the same specific gravity were passed through the centrifuge to flush out any remaining float material. The centrifuge was then switched off and the sink products were removed from the bowl and set aside for analysis. The SG of the salt suspension containing the float product was lowered using tap water (typically in increments of 0.1 SG units) and again fed to the centrifuge. This procedure was repeated until a SG range from 1.2 to 1.7 was investigated.

Figures 55 and 56 provide a summary of the float-sink test results obtained with each coal seam. The raw test data is provided in Appendix A. Due to the high specific gravities of mineral matter pyrite, it was possible to achieve substantial reductions in both 


\section{Elkhorn No. 3 Seam}
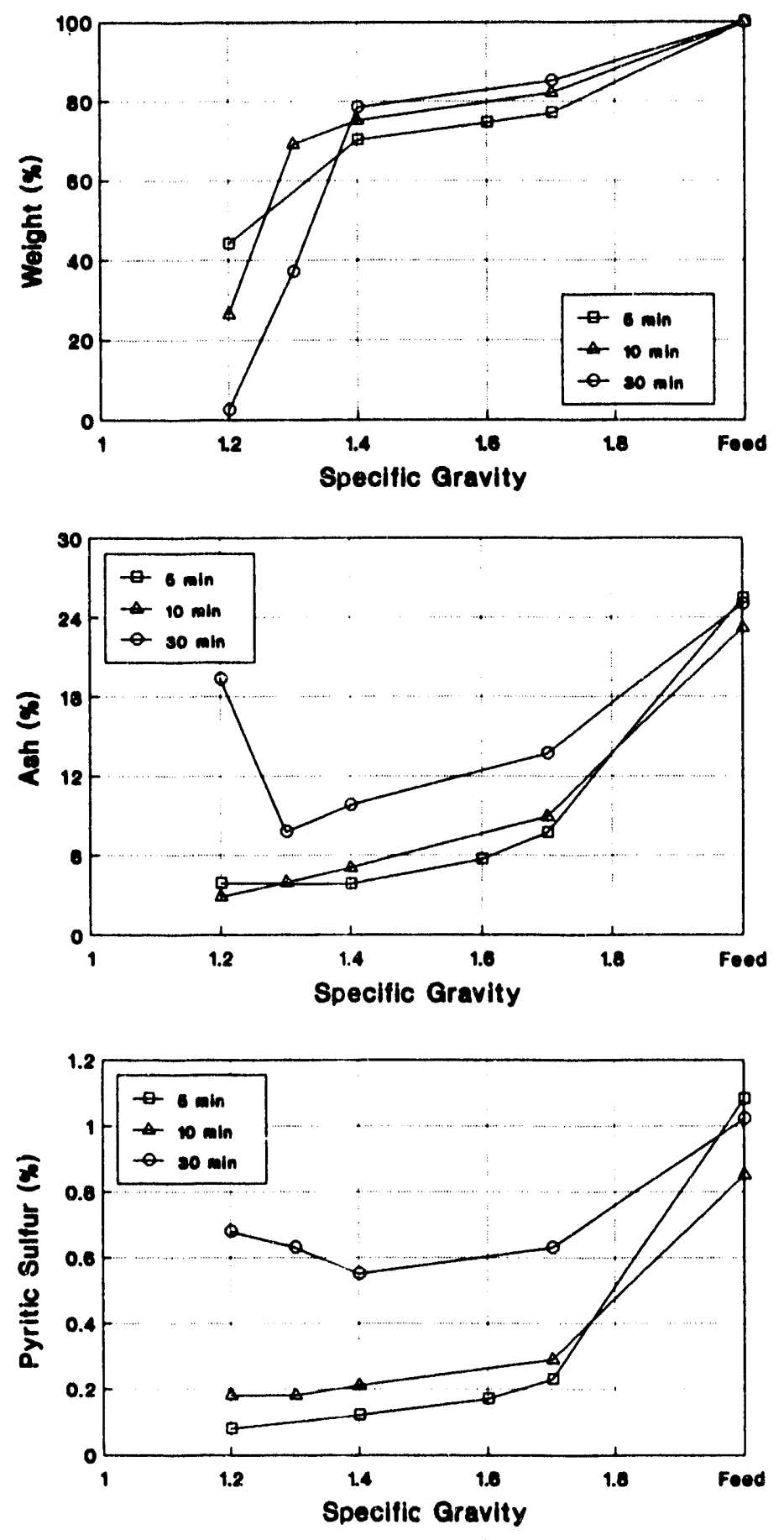

Figure 55. Results of centrifugal float-sink tests conducted on the Elkhorn No. 3 seam after grinding for 5,10 and 30 minutes. 

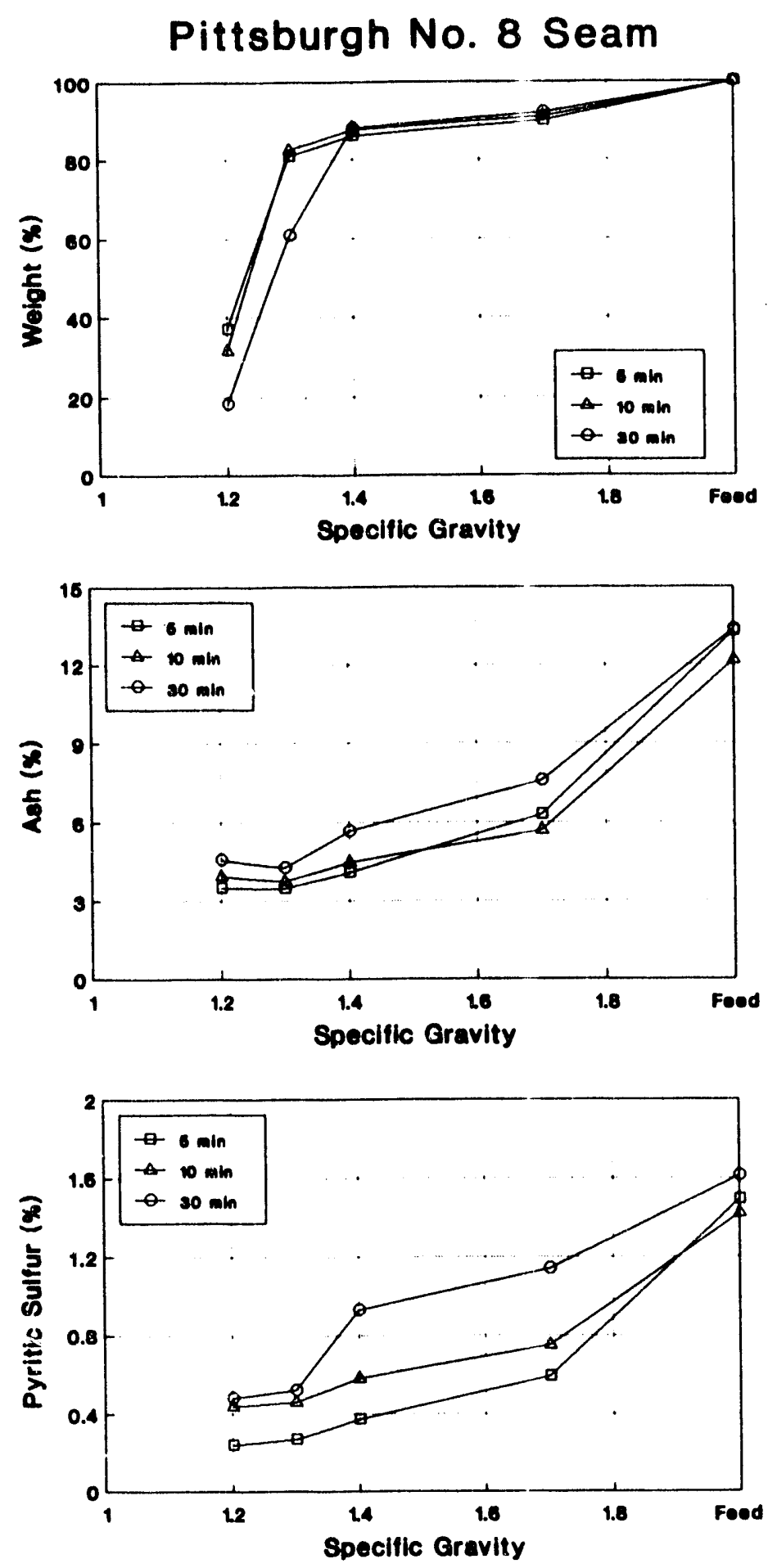

Figure 56. Results of centrifugal float-sink tests conducted on the Pittsburgh No. 8 seam after grinding for 5, 10 and 30 minutes. 
ash and pyritic sulfur for both coals after just 5 minutes of attrition grinding using the float-sink technique. For the case of the Elkhorn No. 3 seam coal, a 1.4 SG separation produced a clean coal containing less than $3.9 \%$ ash and $1 \%$ total sulfur. This level of cleaning corresponds to a carbonaceous recovery of $93 \%$ and a mineral matter rejection of $88 \%$. Processing of the Pittsburgh No. 8 seam coal at 1.3 SG produced less than $3.5 \%$ ash and $3.2 \%$ total sulfur, which corresponds to a carbonaceous recovery of $92 \%$ and rejection of mineral matter approaching $74 \%$.

Unfortunately, the washability results obtained at the finer grind sizes were significantly poorer than those obtained after 5 minutes of grinding. Since liberation generally increases with a finer grind size, this data suggests that the centrifugal separator used in the present work is incapable of handling extremely fine particles. The test data suggests that ultrafine mineral matter (particularly clay) tended to be recovered with the float product in each stage. This possibility explains the very high ash content of the 1.2 SG float product obtained for the Elkhorn No. 3 seam coal in Figure 55. It may also be possible that the high concentrations of salt promoted coagulation of coal particles and an increase in the entrapment of ultrafine mineral matter. It is interesting to note that the entrapment problem was more pronounced for the Elkhorn No. 3 seam coal since it was more easily coagulated than the Pittsburgh No. 8 seam coal. Although nonpolar organic hea $;$, liquids are more costly and difficult to use, these liquids are probably more suitable for coal washability testing since they completely wet the coal surfaces and improve dispersion. In any case, the washability results obtained in this study at the finer sizes (particularly after 30 minutes) are questionable. Neither the SEM-IPS system or centrifugal float-sink tests were capable of accurately determining the degree of liberation of the highly micronized coals used in the present work. 
The liberation results obtained using the SEM-IPS system and washability tests are compared in Figures 57 and 58 for the Elkhorn No. 3 and Pittsburgh No. 8 seams, respectively. In each case, the experimental results were converted into recoveryrejection data so that a common base for comparison existed for each analysis technique. The SEM-IPS results obtained using the Elkhorn No. 3 seam coal predicts a significantly lower rejection of mineral matter than do the float-sirik test results. The reverse trend was observed for the case of the Pittsburgh No. 8 seam coal. This discrepancy can probably be attributed to differences in the size distributions of mineral matter and pyrite size in each coal and variations in clay content. Nevertheless, these results do indicate that mineral matter rejections near $80 \%$ can be achiev'ed at recovery levels approaching $90 \%$ for both coal seams.

\section{Task 3.3 Mixing Studies}

The effects of mixing on coagulation kinetics were studied to determine if the mechanically induced kinetic energy of particles $\left(V_{k}\right)$ can be used to coagulate two particles by overcoming the interaction energy barrier $\left(V_{T, \max }\right)$ and increasing the collision frequency. In order for this strategy to be effective, the rate of coagula growth would have to occur faster than the rate of coagula breakage (as discussed in Task 2.3).

The mixing cell design used in this study is shown in Figure 59. Several different combinations of mixing cells and impeller sizes were used for the agitation tests. The various cell configurations are summarized in Table VIII. The kinetic energy $\left(V_{k}\right)$ of the particles in suspension was calculated using the procedure described previously in Task 2.3 . 


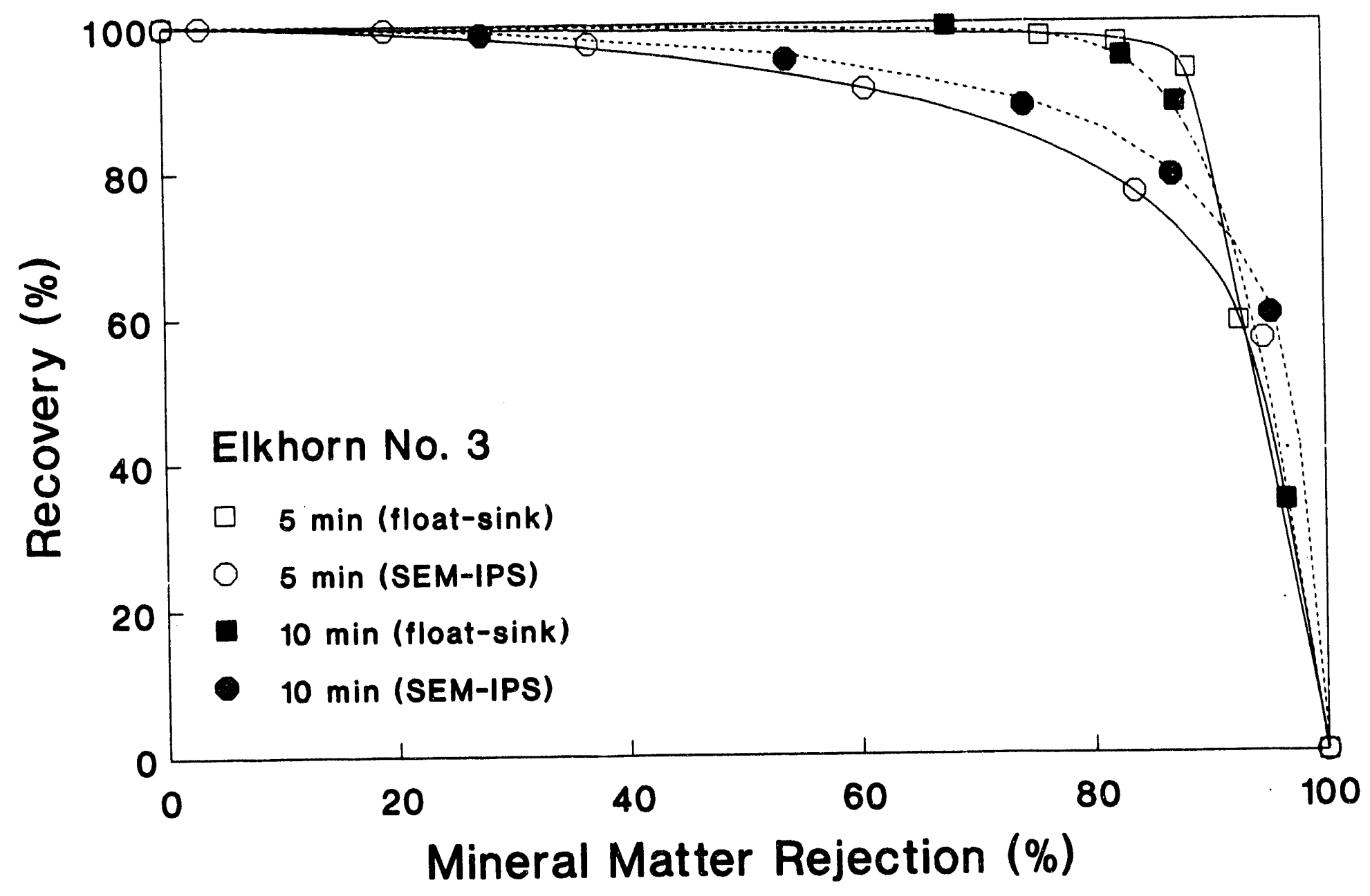

Figure 57. Recovery-rejection curves predicted by SEM-IPS and float-sink techniques for the Elkhorn No. 3 seam after 5 and 10 minutes of grinding. 


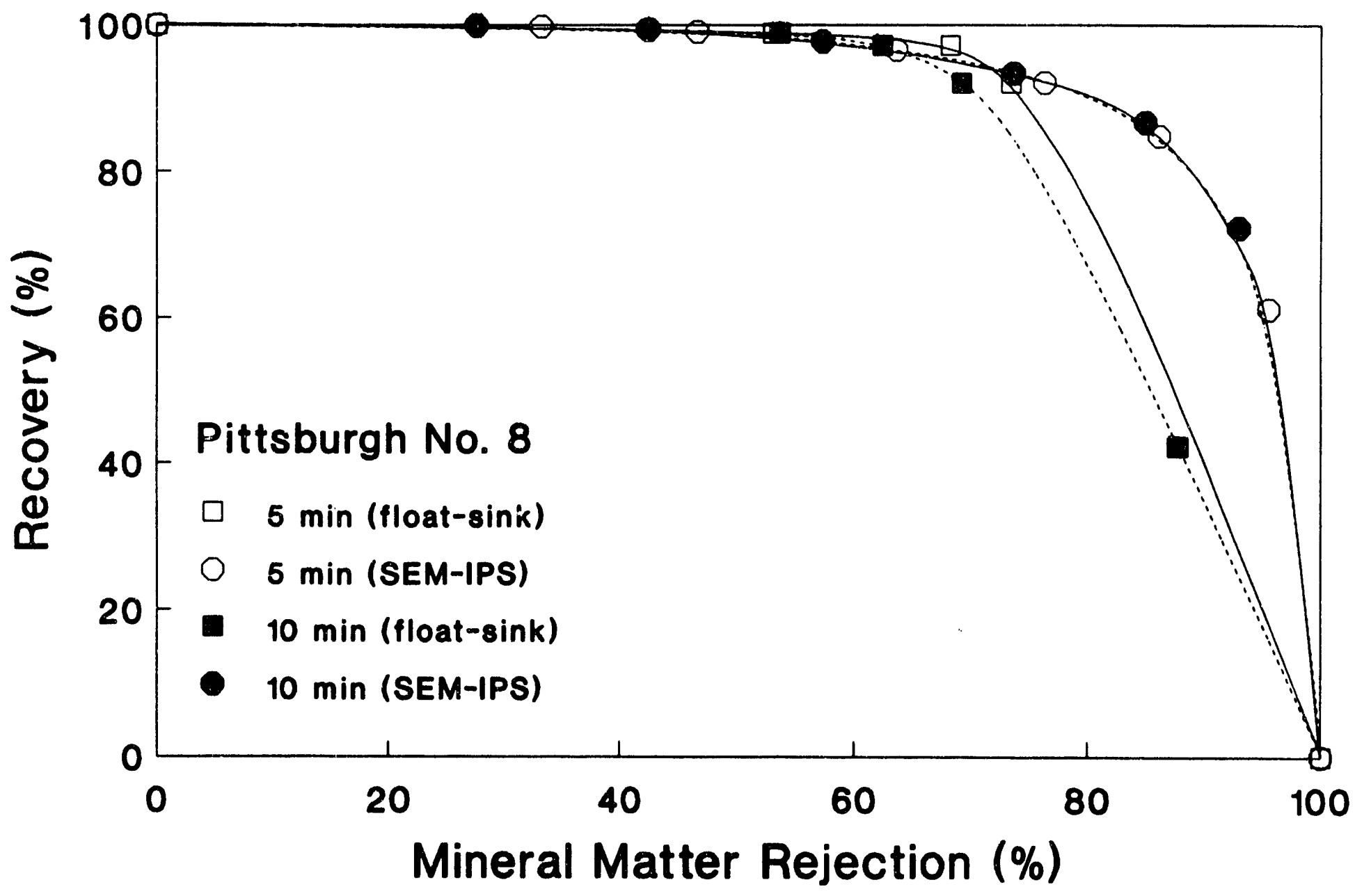

Figure 58. Recovery-rejection curves predicted by SEM-IPS and float-sink techniques for the Pittsburgh No. 8 seam after 5 and 10 minutes of grinding. 

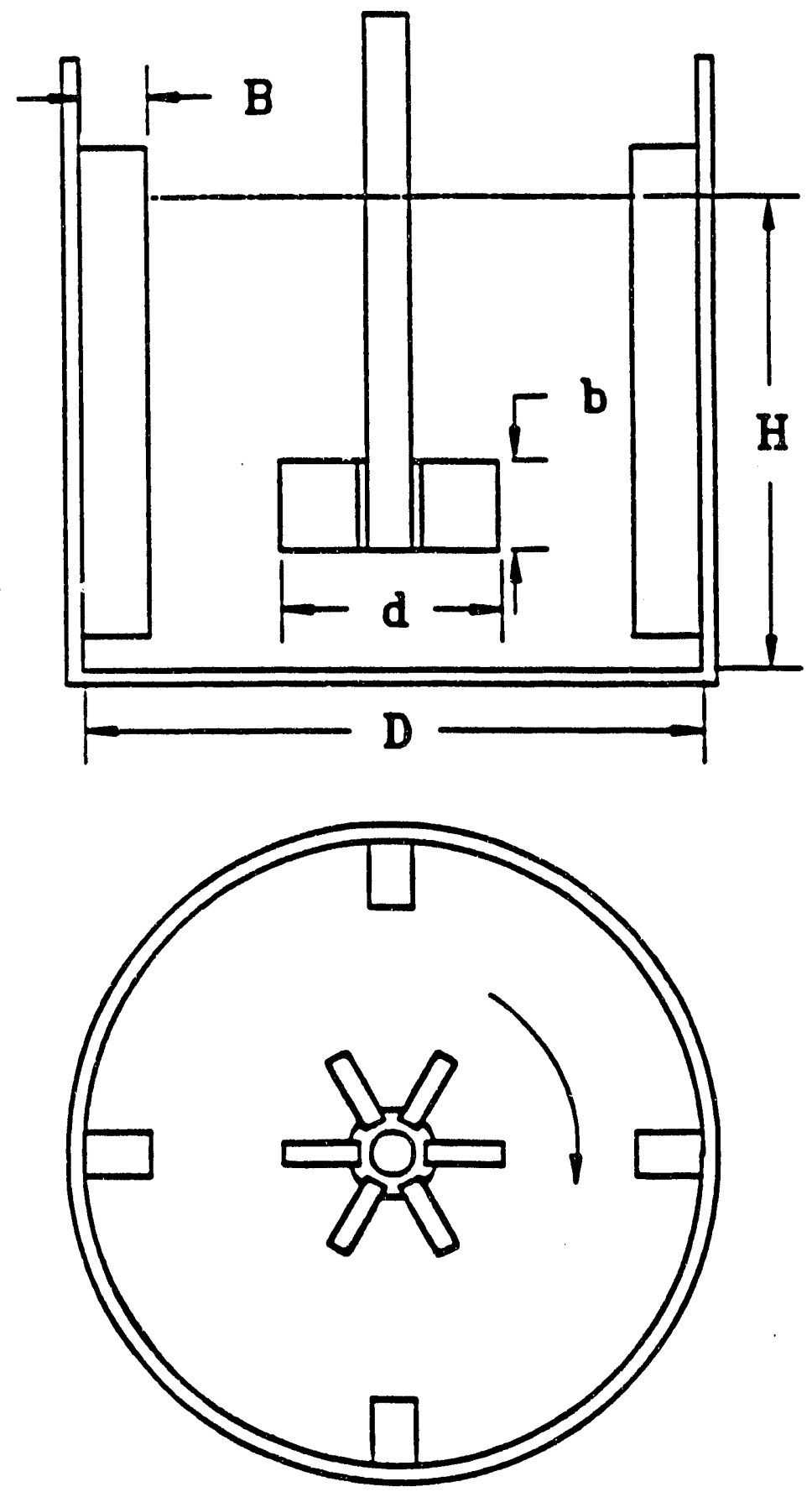

Figure 59. Structure and geometry of the mixer used in the coagulation test work. 
Table VIII. Cell configurations utilized in the SHC mixing studies.

\begin{tabular}{|l|c|c|c|c|}
\hline \multicolumn{1}{|c|}{ Cell } & Cell \#1 & Cell \#2 & Cell \#3 & Cell \#4 \\
\hline \hline Cell Diameter, D (in.) & 5.50 & 5.50 & 5.50 & 4.25 \\
Baffle Width, B (in.) & 0.75 & 0.50 & 0.50 & 0.25 \\
Baffle Number, $\mathrm{n}$ & 4 & 6 & 4 & 8 \\
Pulp Level (in.), $\mathrm{H}$ & 2.75 & 2.75 & 2.75 & 3.25 \\
\hline
\end{tabular}

$\begin{array}{lll}\text { Impeller Width, } \mathrm{b} & =1.25 \text { inches } \\ \text { Impeller Diameter, } \mathrm{d} & =1.5,2,3 \text { inches }\end{array}$

The mixing tests were conducted on a pre-cleaned Elkhorn No. 3 coal sample having an ash content of approximately $3 \%$. The coal was ground in an attrition mill for 5 minutes which resulted in a mean particle size of approximately $4.7 \mu \mathrm{m}$. The tests were performed using a $10^{-3} \mathrm{M} \mathrm{KCl}$ solution at a solids concentration of $1.5 \%$ by weight.

The mixing tests involved measuring the mean coagula size using a Lasentec particle size analyzer for several combinations of mixer geometries and agitation speeds. The tests were conducted at $\mathrm{pH}$ values of 7.5 and 8.4. At pH 7.5, the interaction energy between coal particles is purely attractive due to the hydrophobic interaction energy, i.e., there is no energy barrier to hinder coagulation. Thus, the kinetic energy supplied by mechanical agitation could only increase the coagulation rate by an increase in collision frequency. The results of the mixing tests conducted at $\mathrm{pH} 7.5$ are plotted as a function of mean coagula size versus $V_{k}$ in Figure 60 for the five different configurations examined in the present work. As shown, an increase in $V_{k}$ resulted in a sharp decrease in the coagula $D_{50}$. This result indicates that, for this $\mathrm{pH}$ condition, an increase in $V_{k}$ 


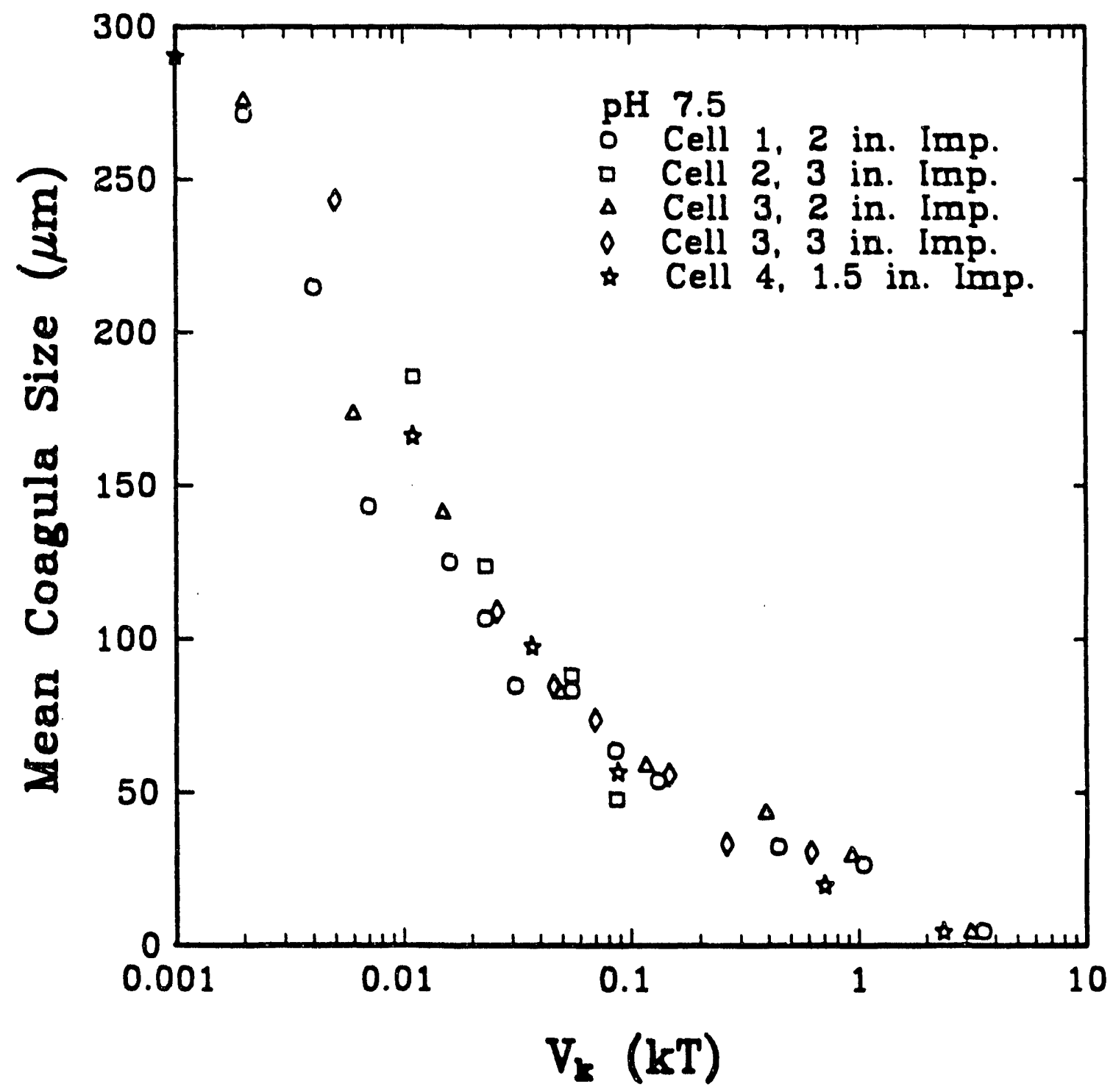

Figure 60. Effect of particle kinetic energy $\left(V_{k}\right)$ on the mean coagula size for various cell-impeller configurations at $\mathrm{pH}$ 7.5. 
enhances coagula breakage rather than promoting coagula growth.

At a pH value of 8.4, an energy barrier of $96 k \mathrm{~T}$ exists between the coal particles due to an increase in the repulsive electrostatic component. Thus, mixing tests were conducted at this $\mathrm{pH}$ to determine the possibility of using the kinetic energy induced by agitation to overcome the energy barrier and to improve the coagulation rate. As shown in Figure 61, an increase in the applied $V_{k}$ again decreased the mean coagula size. Unfortunately, these results indicate that the coagula breakage rate increases faster than the coagula growth rate when mechanical agitation is increased.

\section{Task 3.4 Development of Methods for Enhancing the Separation of Coagula}

One of the shortcomings of the selective hydrophobic coagulation process is that the resulting coagula size is relatively small. The small coagula size results in relatively slow settling rates and low throughput capacities, which in turn will incur higher capital and operating costs. This task investigates various approaches to the enhancement of coagula size and, hence, recovery rates.

\section{Task 3.4.1 Addition of Coarse Particles}

One possible method of improving the settling kinetics is to add coarse coal particles. The advantages of this approach are: 1) coagulation kinetics are improved by coagulating coarse particles with ultrafine particles and 2) the resulting coagula size is larger: thus increasing the settling rate.

Varying amounts of coarse particles were added to the Elkhorn No. 3 seam coal in an effort to improve the settling kinetics of the selectively coagulated coal particles. The coarse particles were obtained by screening a dry-ground sample obtained from the Buller 


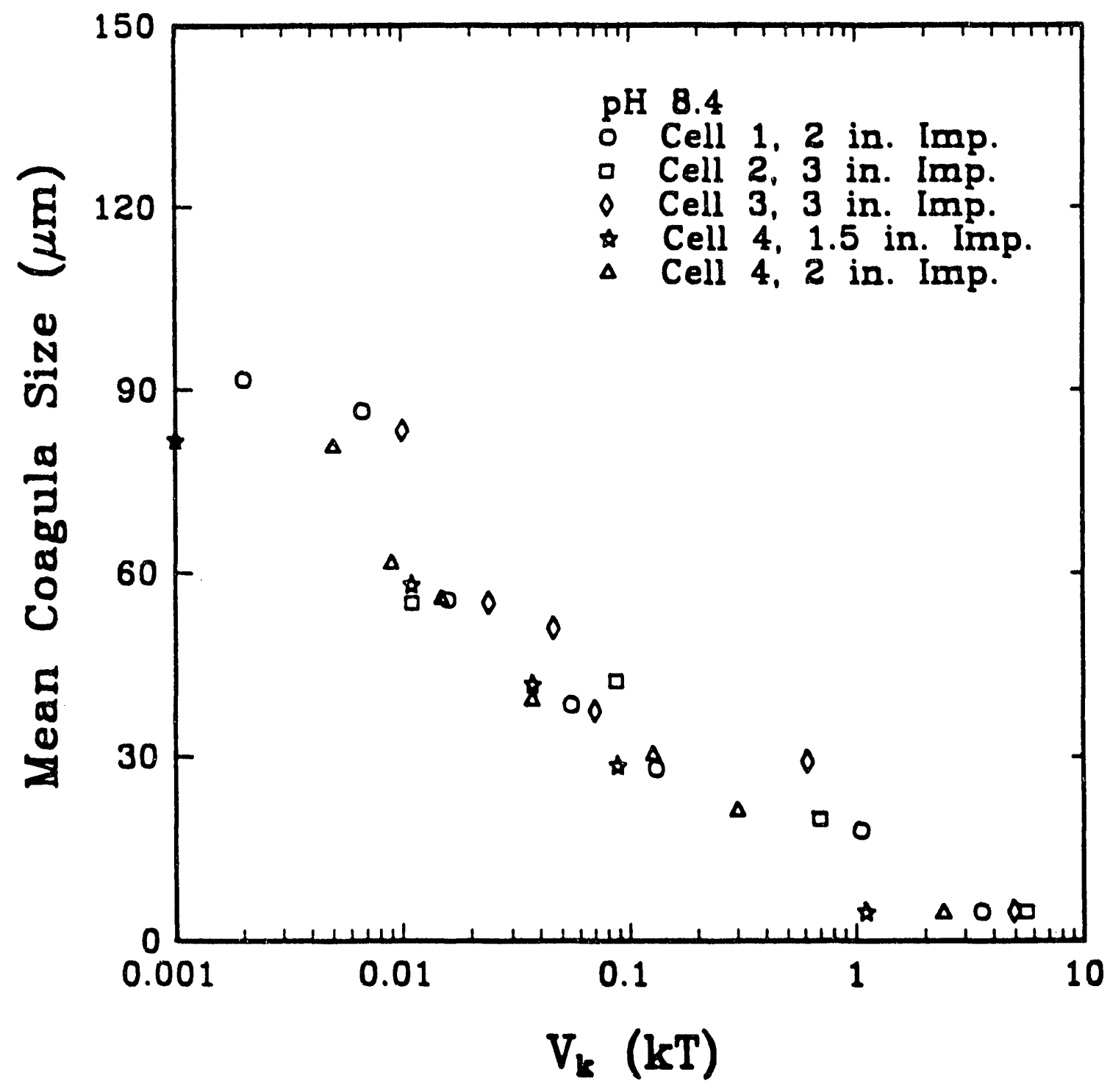

Figure 61. Effect of particle kinetic energy $\left(V_{k}\right)$ on the mean coagula size for various cell-impeller configurations at $\mathrm{pH} 8.4$. 
seam, New Zealand. This sample was selected since it provided a very hydrophobic material containing very little ash as mined. The size classes examined include $200 \times 270$, $270 \times 325$, and $325 \times 400$ mesh. The micronized coal slurries were obtained by attrition grinding a precleaned Elkhorn No. 3 coal seam sample for 30 minutes, which resulted in a $D_{s o}$ of approximately $3 \mu \mathrm{m}$. Distilled water was used to maintain a $1 \%$ solids concentration by weight.

The effect of coarse particle additions was obtained by measuring the settling distance as a function of time in a graduated cylinder $(1000 \mathrm{ml})$. Prior to each test, the $\mathrm{pH}$ of the slurry was adjusted to a value of 7.0 by either the addition of sodium hydroxide or hydrochloric acid. After the $\mathrm{pH}$ reached equilibrium, the coal slurry was placed into a graduated cylinder $(1000 \mathrm{ml})$ which was inverted 5 times. The settling time was started upon completion of the inversion step. The settling distance was monitored by referencing the position of the "mudline" which forms due to the settling coagula.

Initial experiments were aimed at determining the effect of solids concentration on coagula settling rate. These tests did not involve the addition of coarse seed particles. As shown in Figure 62, the settling distance for a given time interval increased with decreasing solids concentration. The initial settling rate for each solids concentration can be obtained by determining the slope of the settling distance versus time plot during the first 10 minutes. After the first 10 minutes, the slope of the curves tends to decrease due to the effect of hindered settling as the coagula starts to compact. Settling rates of the coagula are plotted as a function of solids concentration in Figure 63 . The results indicate that coagula settling rate increases almost exponentially as the solids concentration is decreased. The settling rate at a solids concentration of $3 \%$ by weight was less than $0.40 \mathrm{~cm} / \mathrm{min}$, whereas the settling rate was greater than $1.60 \mathrm{~cm} / \mathrm{min}$ at a solids content of $0.5 \%$. These trends illustrate the large effect that hindered settling has 


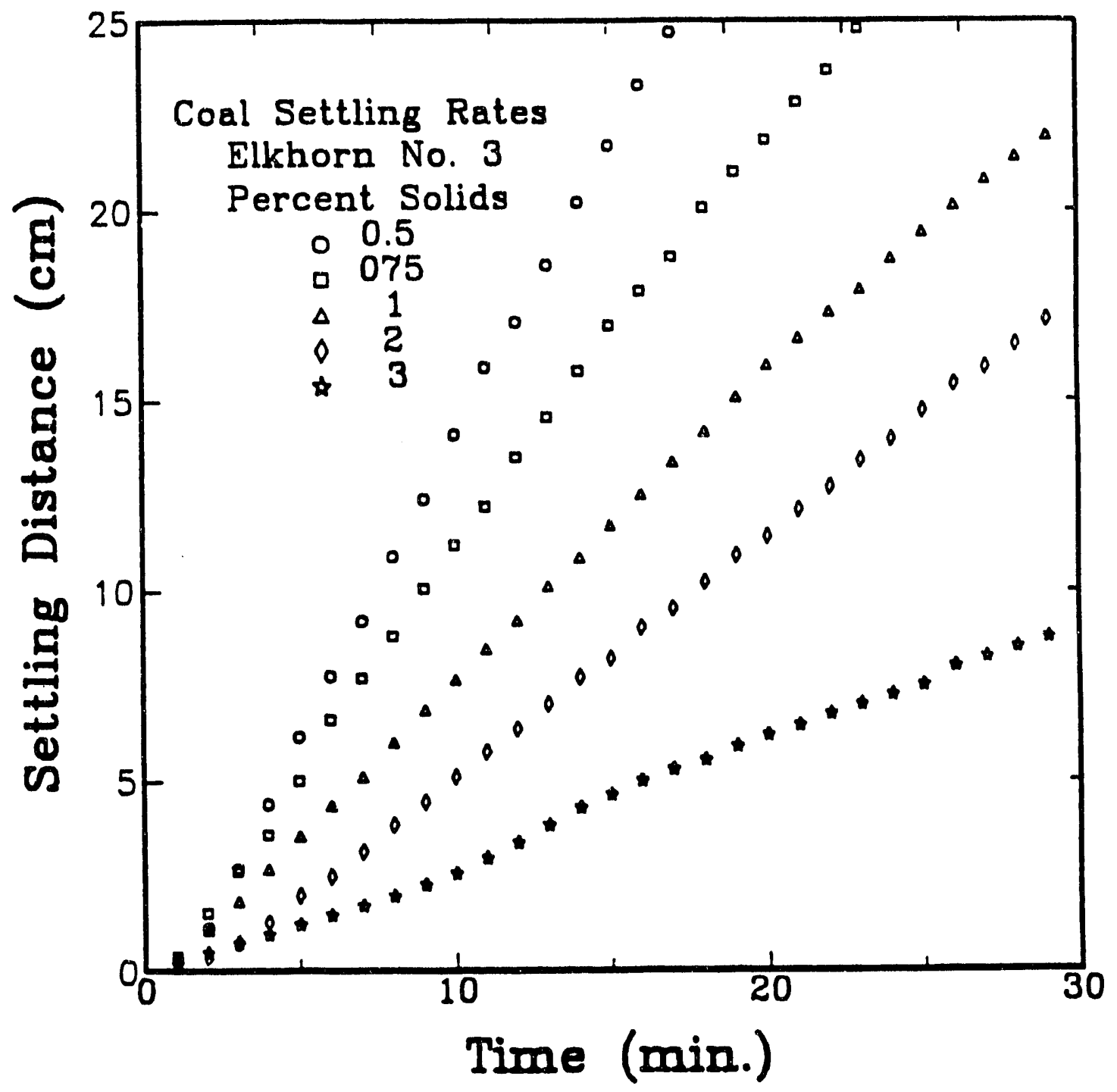

Figure 62. Effect of solids content on the coagula settling behavior for the Elkhorn No. 3 seam coal. 


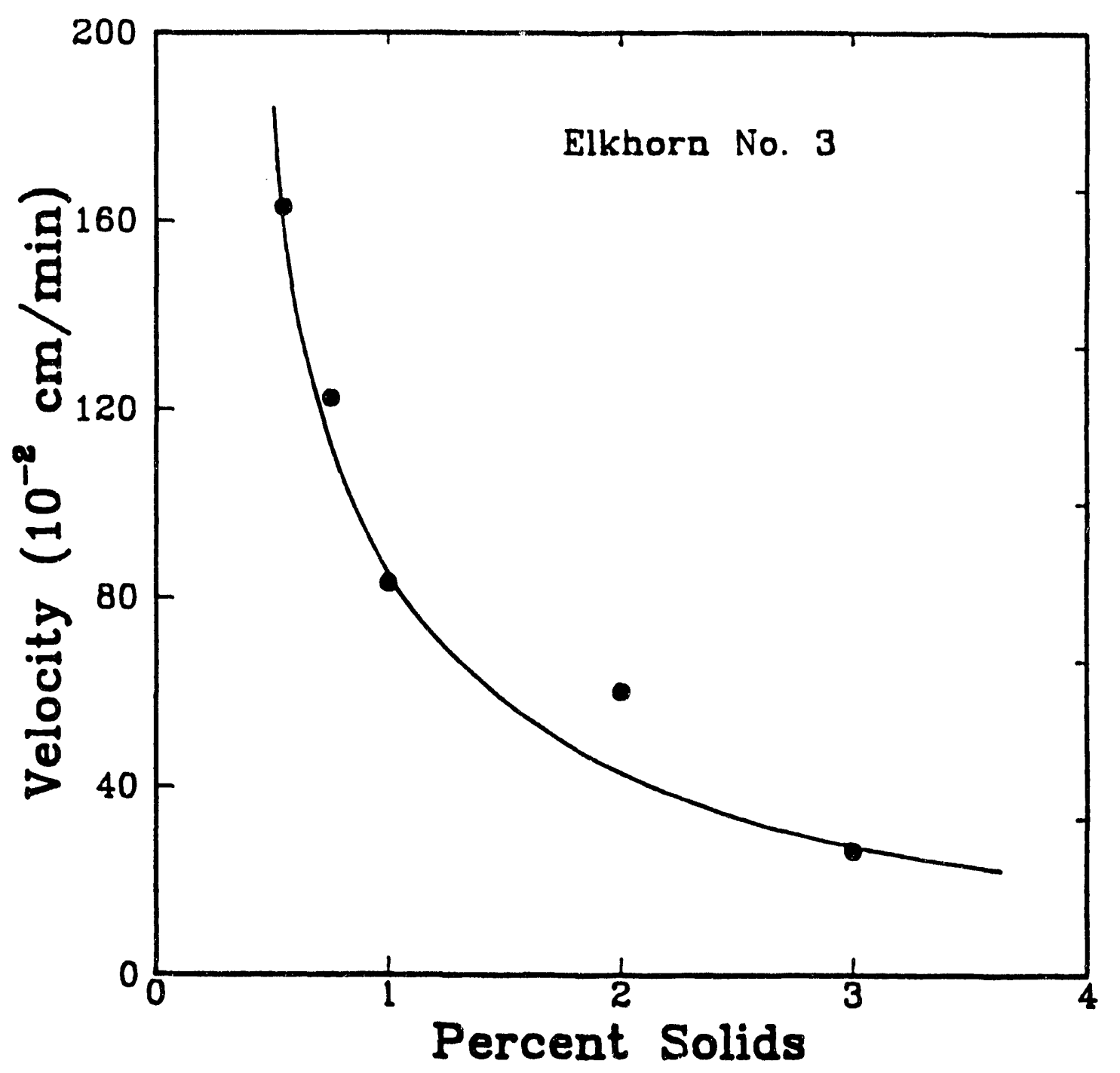

Figure 63. Settling velocity of the coagula as a function of solids content for the Elkhorn No. 3 seam coal. 
on settling rates of coal coagula.

The method of improving the settling rate of coagula at the higher solids concentrations may be to add coarse seed particles. The addition of $200 \times 270$ mesh Buller coal was tested over concentrations of $0 \%$ to $50 \%$ by volume of total dry solids. Coarse particle additions were added based on a volume percent to normalize the effect hindered settling. The solids concentration in the slurry was maintained at $1 \%$ for each coarse seed particle addition. As shown in Figure 64, the settling distance over an incremental period of time increased with increasing concentrations of $200 \times 270$ mesh seed particles. After 15 minutes of settling, the coarse seed addition of $40 \%$ improved the settling distance of the coagula from $12 \mathrm{~cm}$ to $20 \mathrm{~cm}$. Similar trends have been obtained for the $270 \times 325$ and 325x400 mesh size fractions as shown in Figures 65 and 66.

Settling rates for the different size fractions and addition amounts were obtained by measuring the slope of the various settling distance versus time curves over the first 10 minutes of settling. The settling rates shown plotted as a function of coarse particle additions in Figure 67 indicate that the increase in settling rates with increasing coarse particle additions is independent of the size class. The figure also shows that the settling rate was more than doubled from $0.78 \mathrm{~cm} / \mathrm{min}$ to $1.70 \mathrm{~cm} / \mathrm{min}$ after the coarse seed addition of $50 \%$ by volume. While this is a significant improvement in settling rate, the coarse particle addition rate is probably too high for all but a few selected cases.

\section{Task 3.4.2 Addition of Hydrophobized Magnetite}

Another method for improving the settling kinetics of the selective coagulation process may be to add magnetite that has been made hydrophobic by treating it with a collector to the micronized coal slurry. Improved kinetics would be a result of the coal 


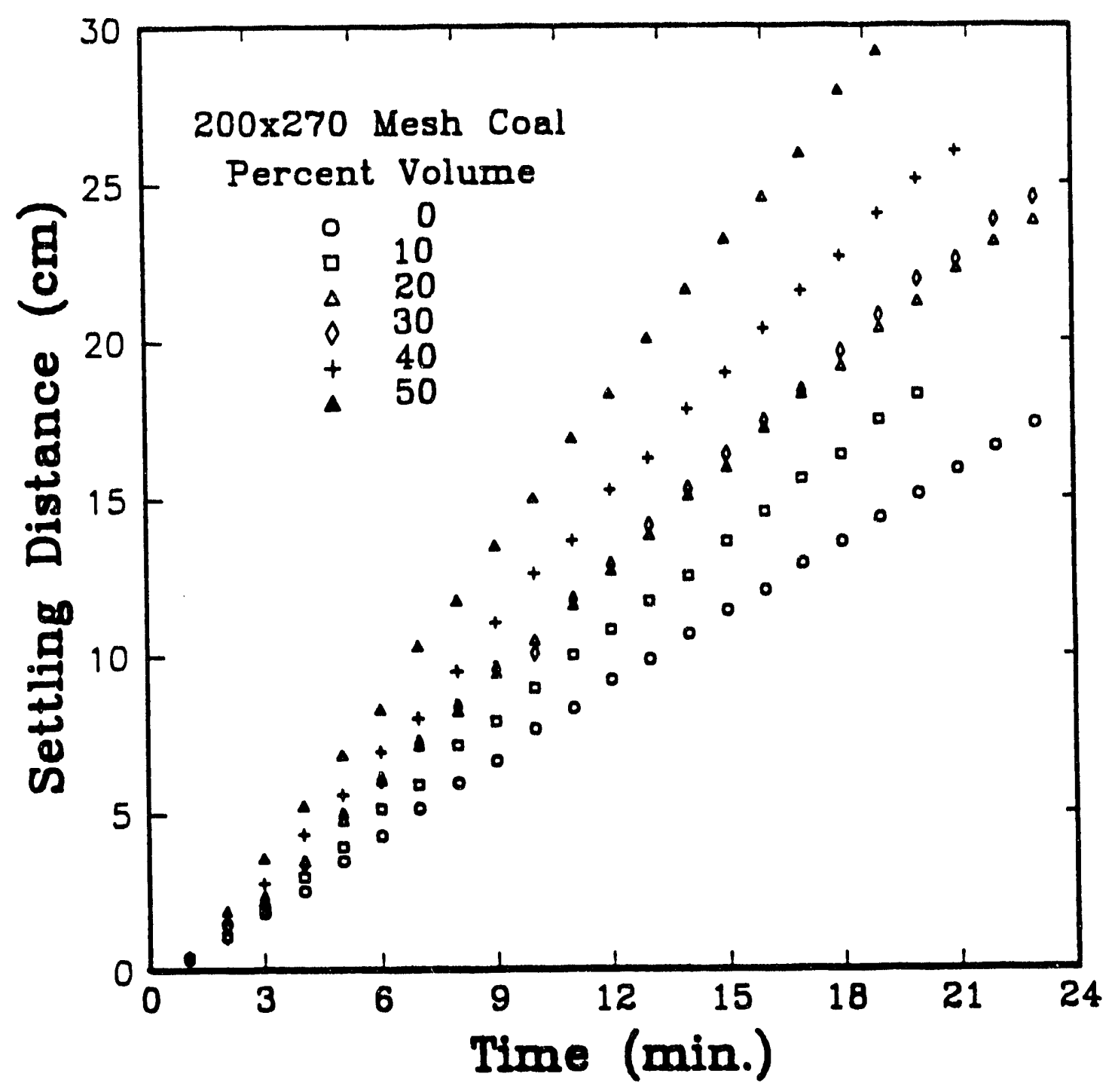

Figure 64. Coagula settling behavior obtained after adding 200×270 mesh Buller seam to micronized Elkhorn No. 3 seam coal. 


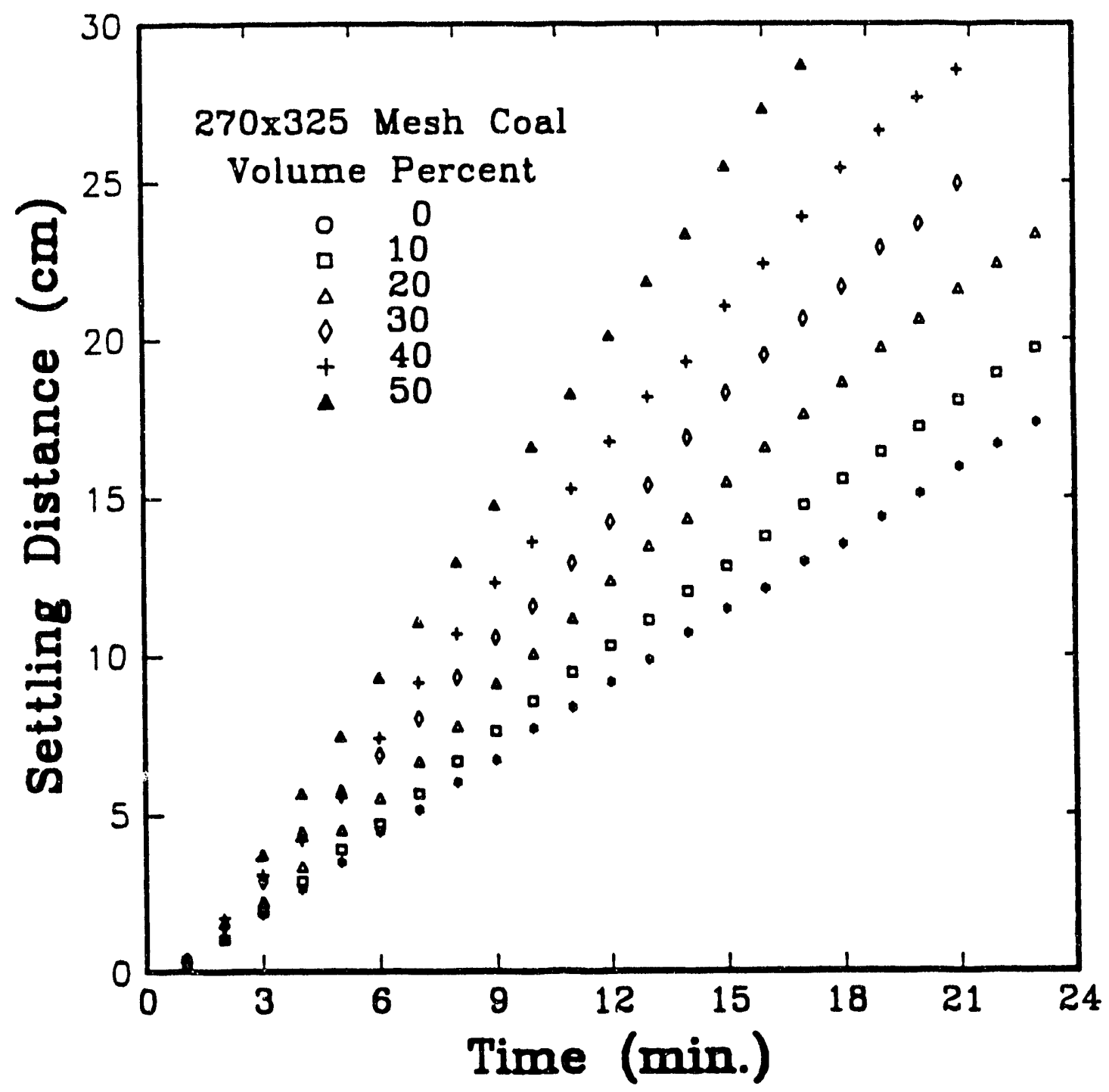

Figure 65. Coagula settling behavior obtained after adding $270 \times 325$ mesh Buller seam to micronized Elkhorn No. 3 seam coal. 


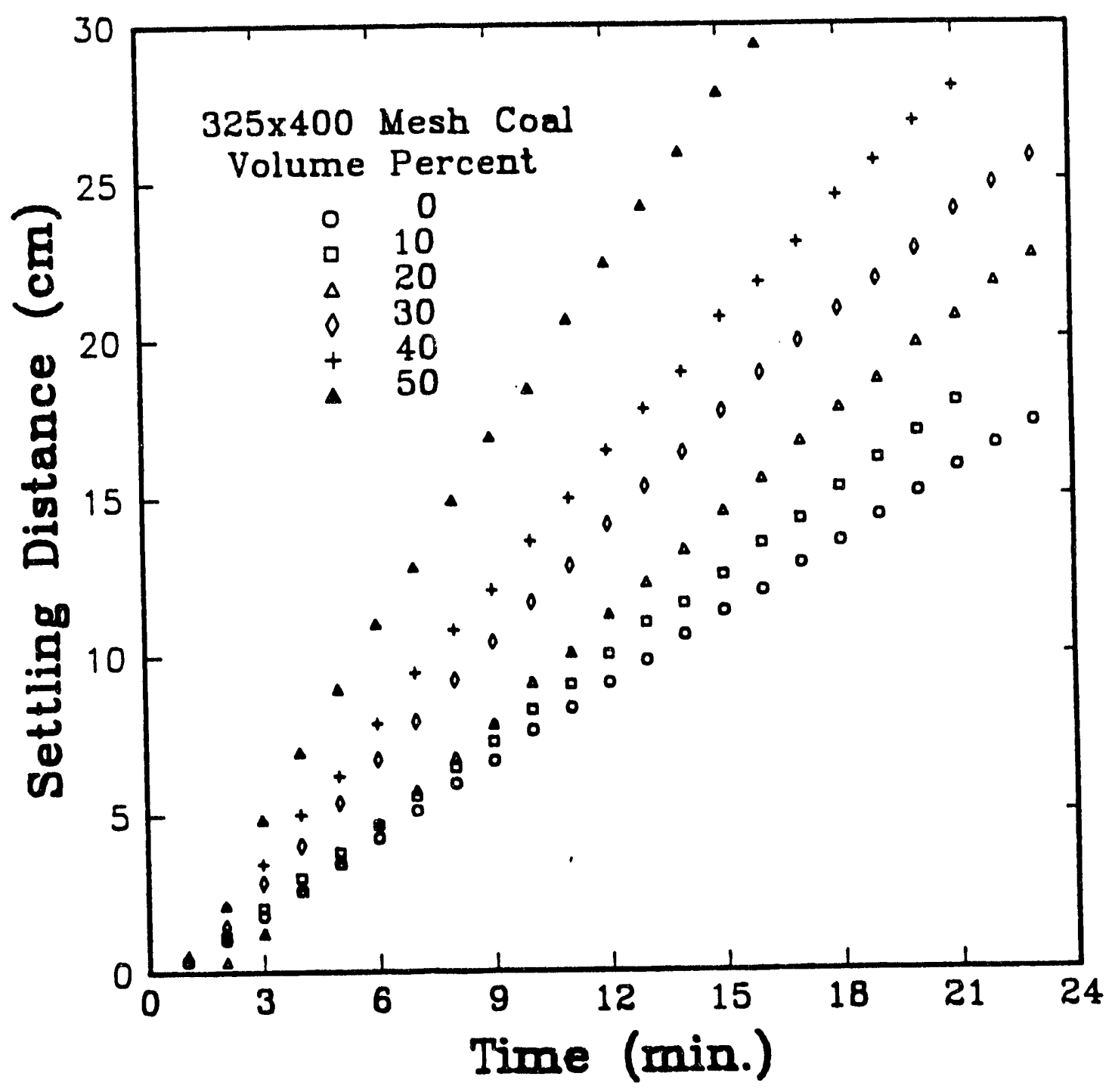

Figure 66. Coagula settling behavior obtained after adding $325 \times 400$ mesh Buller seam to micronized Elkhorn No. 3 seam coal. 


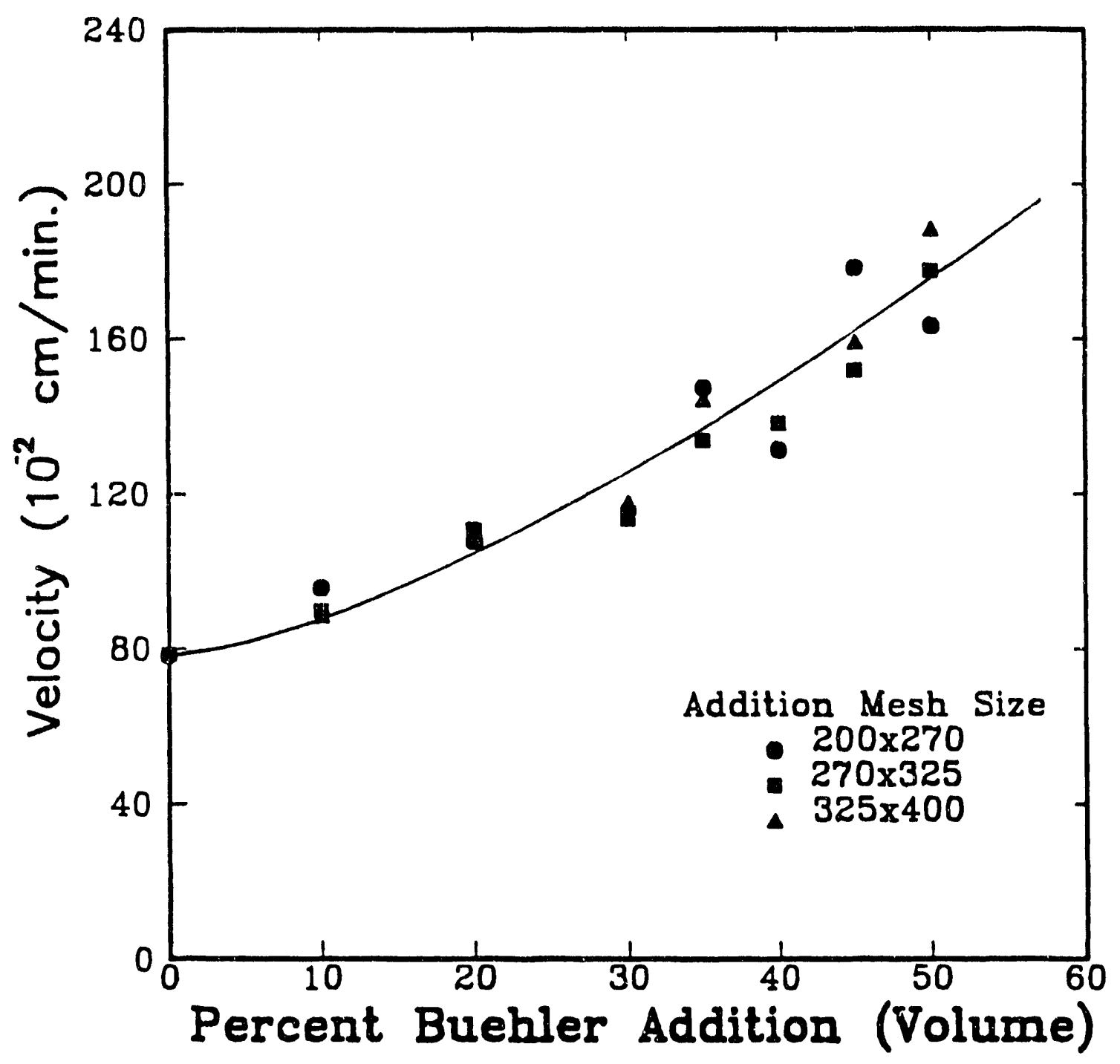

Figure 67. Coagula settling velocity obtained after adding various size fractions of Buller coal to the Elkhorn No. 3 seam coal. 
attaching to the heavier (s.g. =5.2) hydrophobic magnetite. As discussed above, coarse seed particles of hydrophobic magnetite may also be beneficial for improving the settling kinetics.

In order to test this hypothesis, various size fractions and amounts of hydrophobic magnetite were added to a finely-ground coal slurry. As in Task 3.4.1, an Elkhorn No. 3 seam coal which had been micronized for 30 minutes in a stirred ball mill was used in all tests. Chunks of magnetite were obtained from Ward's Natural Science Establishment, crushed and screened to size fractions of $200 \times 270,270 \times 325$, and $325 \times 400$ mesh, and upgraded by a magnetic separation technique. The surface of the magnetite was rendered hydrophobic by conditioning the various size fractions in a $10^{-4} \mathrm{M}$ octyl hydroxamate solution for 5 minutes. Afterward, the sample was filtered and air-dried. The hydrophobicity of the treated magnetite was tested by performing test tube flotation tests. If the magnetite was non-floatable, the sample was re-treated using the procedure described above.

Settling kinetic tests were conducted over a range of concentrations (i.e., 0\%-50\% by volume) for each size class that was added to the coal slurry. The results are shown plotted as settling distance versus time in Figures 68,69 and 70. The test results show that the settling distance for an incremental time increased as the magnetite addition was increased. The settling rates corresponding to each size class were obtained from the slope of the settling distance versus time curves. These results are plotted as a function of percent magnetite addition in Figure 71. The results indicate that the settling rate increases with increasing magnetite additions. A magnetite addition of $50 \%$ by volume improved the settling rate from 0.78 to $1.55 \mathrm{~cm} / \mathrm{min}$. The increase in settling rate was independent of the size class, which is similar to the trend for the coarse coal additions discussed above. 


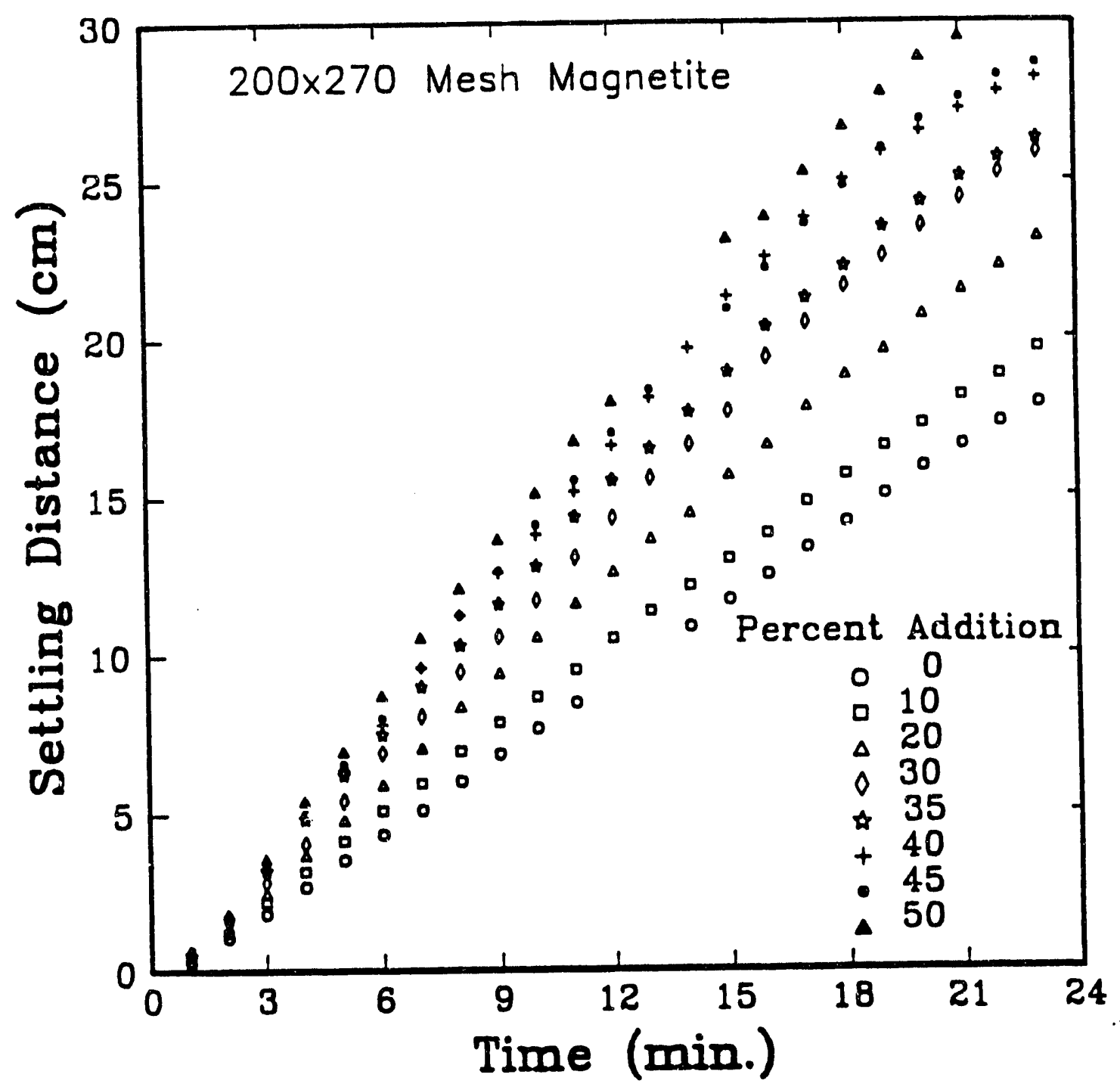

Figure 68. Coagula settling behavior obtained after adding 200×270 mesh magnetite to micronized Elkhorn No. 3 seam coal. 


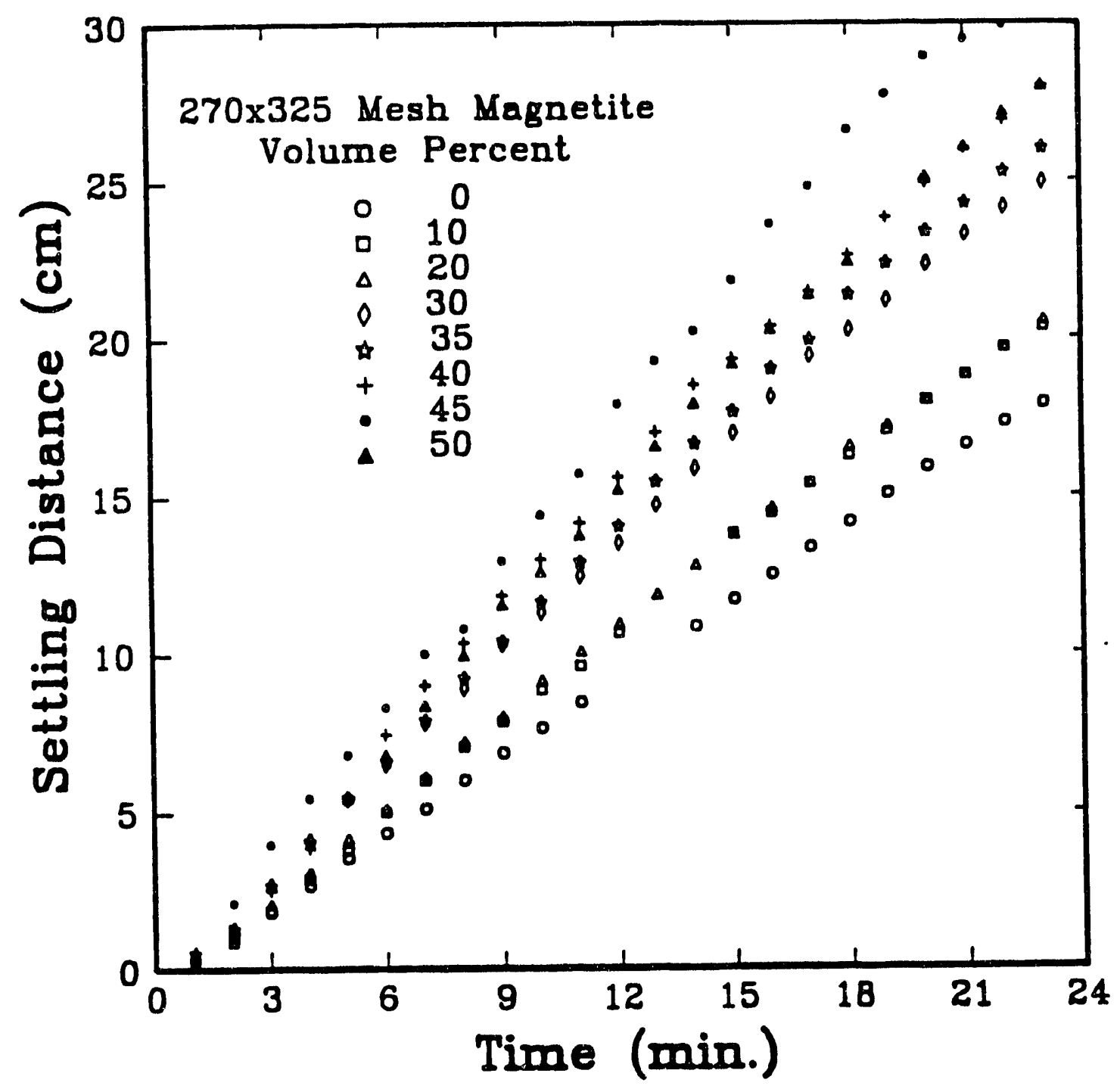

Figure 69. Coagula settling behavior obtained after adding 270x325 mesh magnetite to micronized Elkhorn No. 3 seam coal. 


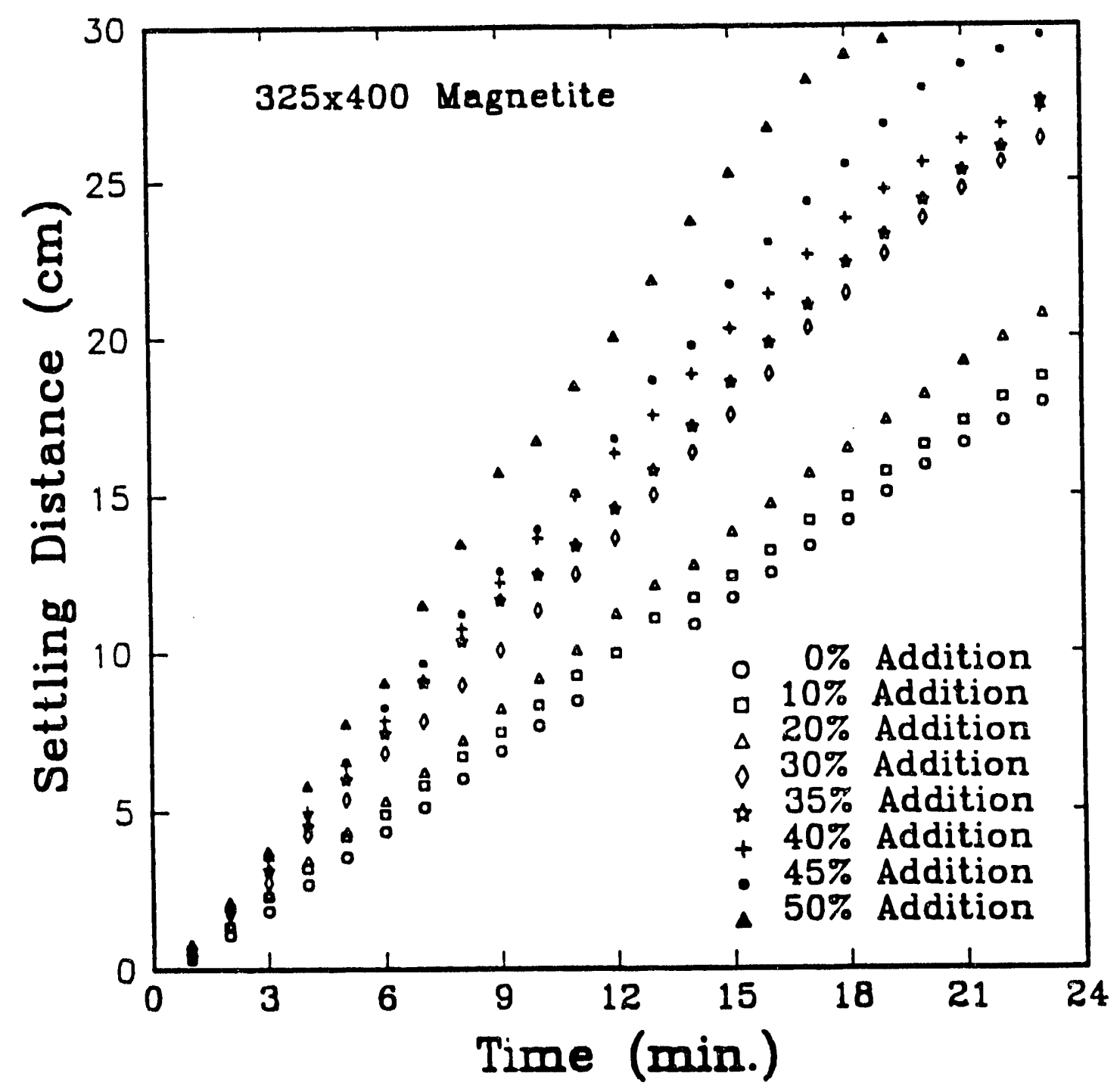

Figure 70. Coagula settling behavior obtained after adding $325 \times 400$ mesh magnetite to micronized Elkhorn No. 3 seam coal. 


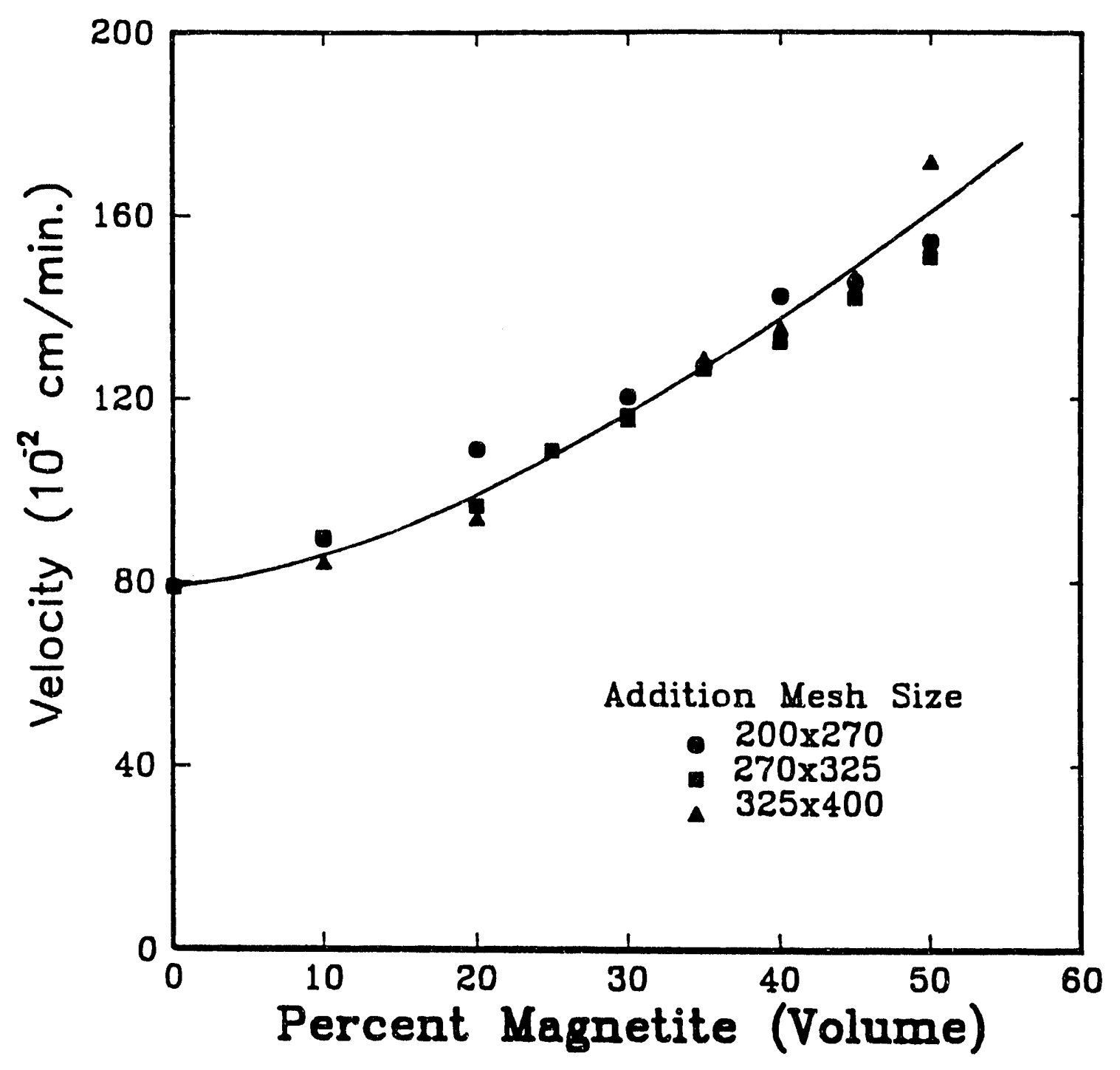

Figure 71. Coagula settling velocity obtained after adding various size fractions of magnetite to the Elkhorn No. 3 seam coal. 
It is interesting to note that the settling rates from the coarse coal additions were slightly greater than those obtained from the hydrophobic magnetite additions. This phenomenon may be due to a lower coagulation efficiency for the magnetite-coal interactions resulting from shape effects, impurities in the magnetite, lower hydrophobicity of the treated magnetite, or a larger electrostatic repulsive force between coal and magnetite particles. However, since the magnetite is recoverable and can be reused, the higher addition rates may be acceptable since significant increases in throughput can be achieved using this technique. Further tests are warranted to establish the efficiency of the magnetite recovery step.

\section{Task 3.4.3 Addition of a Hydrocarbon Oil}

A third possible method of improving settling rates is to increase the coagula size by adding small amounts of hydrocarbon oil. When used in small quantities, this oil may increase the size of the coagula by enhancing the hydrophobicity of the coal. In this study, tests were conducted to determine how the addition of kerosene, diesel fuel, and a coal-oil byproduct from a coal gasification process might affect coagula size. These experiments were carried out at $\mathrm{pH} \mathbf{8 . 0 ,}$ which is close to the critical $\mathrm{pH}$ for the coagulation of Elkhorn No. 3 coal. The hydrocarbons were added as emulsions containing $0.08 \%$ oil by mass in distilled water. Each coal sample was ground in an attrition mill for 30 minutes to produce a $3.5 \mu \mathrm{m}$ mean size and then transferred to the mixing vessel. Distilled water was used to adjust the slurry solids content to $1.5 \%$, and the $\mathrm{pH}$ was adjusted to the desired level with either $\mathrm{NaOH}$ and $\mathrm{HCl}$. After the $\mathrm{pH}$ stabilized, the desired amount of oil emulsion was injected by a syringe into the agitated slurry. 
The results of the hydrocarbon addition experiments are summarized in Figures 71-74. In each series of tests, coagula size was determined as a function of agitation intensity for various dosages of each hydrocarbon emulsion. In each figure, the mean coagula diameter measured using the Lasentec analyzer was plotted versus the calculated kinetic energy of mixing $\left(V_{\mathrm{k}}\right)$. Unfortunately, the test data shown in Figures 72,73 and 74 indicate that there was considerable scatter in the measured coagula size distributions. In order to eliminate the possibility that the scatter in the data might be a result of conducting the tests near the critical $\mathrm{pH}$, a second series of tests was carried out at $\mathrm{pH}$ 7.45. Only two oils, kerosene and diesel, were used in the second series of tests. As shown in Figures 75 and 76, the test data obtained in this series of tests also display considerable scatter. These results suggest that the problem of data scatter is inherent to the measurement technique itself.

Despite the significant degree of data scatter, several important conclusions can be drawn from the test results shown in Figures 72-76. First, the test results demonstrate that mixing intensity adversely affects coagula size. In all cases studied, the mean coagula size decreased sharply as the kinetic energy of mixing increased. This suggests that the coagula formed by the SHC process are relatively weak and that the turbulence within the separator must be kept to a minimum to prevent breakup of the coagula. The test results also indicate that the effects of the hydrocarbon additions on coagula size are less pronounced as the agitation intensity increases. At high agitation intensities (e.g., greater $0.1 \mathrm{kT}$ ), the measured coagula size becomes relatively constant regardless of the hydrocarbon dosage. At lower agitation intensities, no clearly discernible relationship between hydrocarbon addition and coagula size can be established due to the increased data scatter. However, in nearly every case studied, the largest coagula were obtained in the absence of hydrocarbon additions. This finding suggests that the hydrocarbon oils 


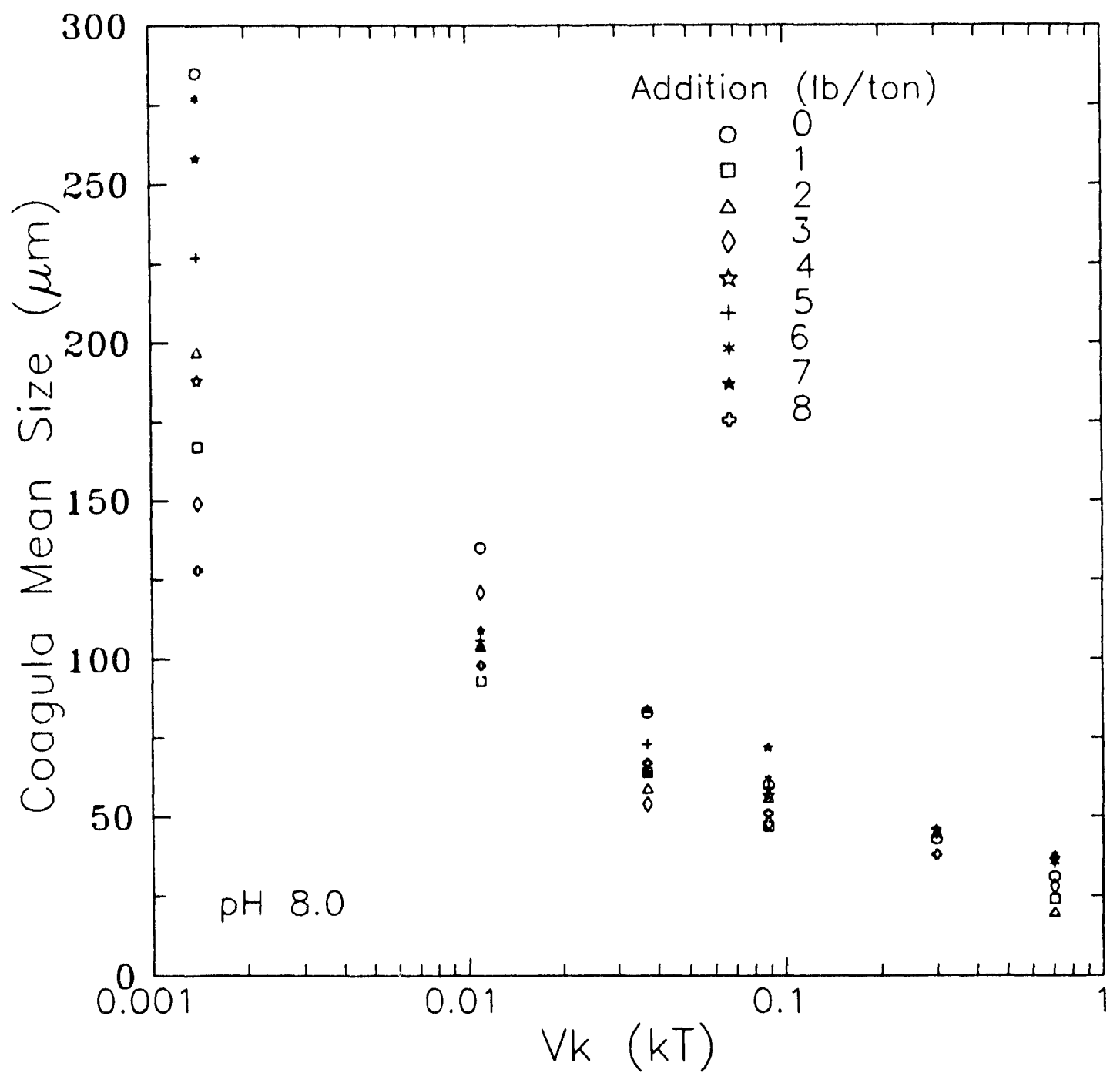

Figure 72. Effect of kerosene addition and mixing intensity on mean coagula size for Elkhorn No. 3 seam coal. 


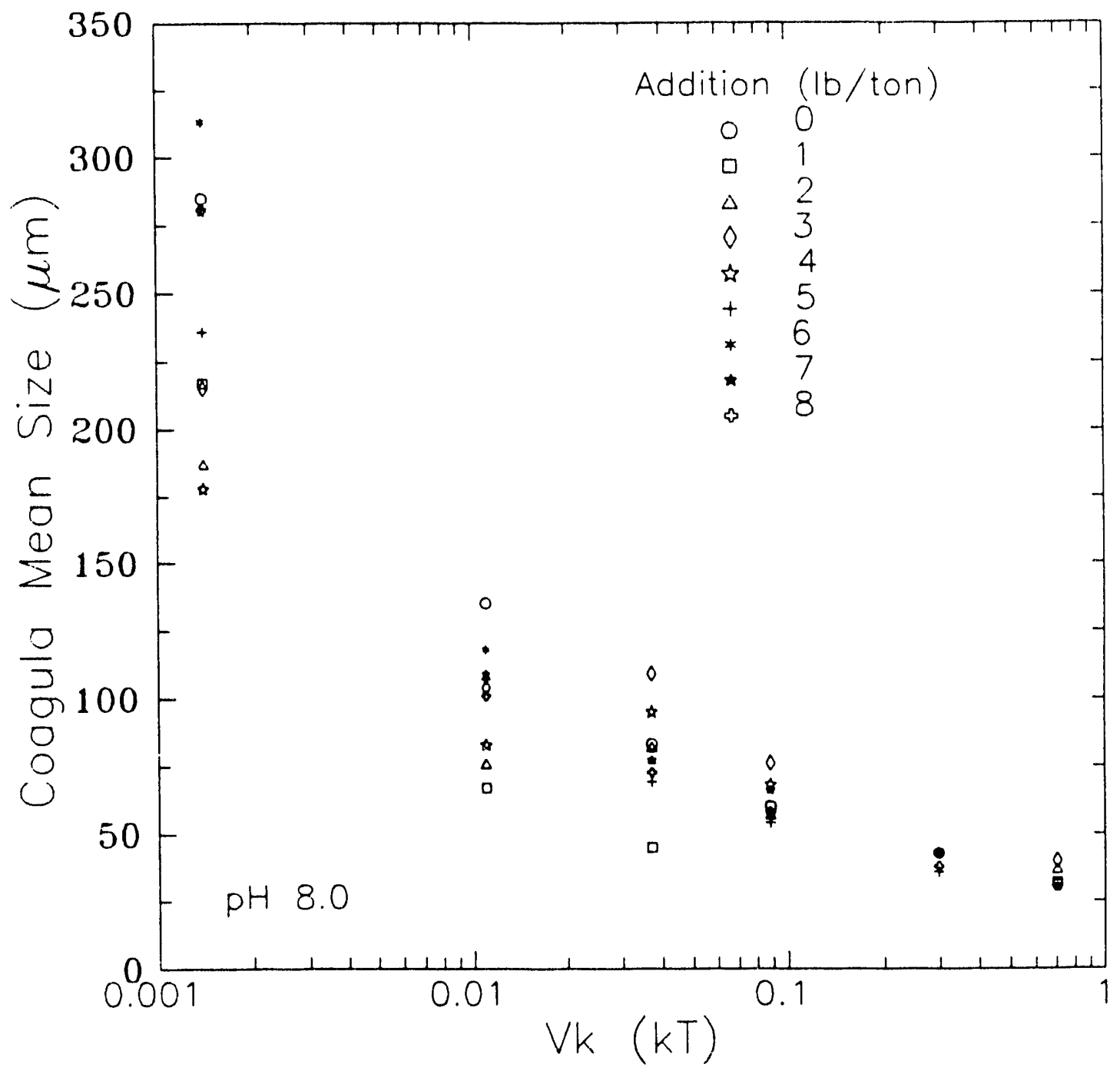

Figure 73. Effect of diesel addition and mixing intensity on mean coagula size for Elkhorn No. 3 seam coal. 


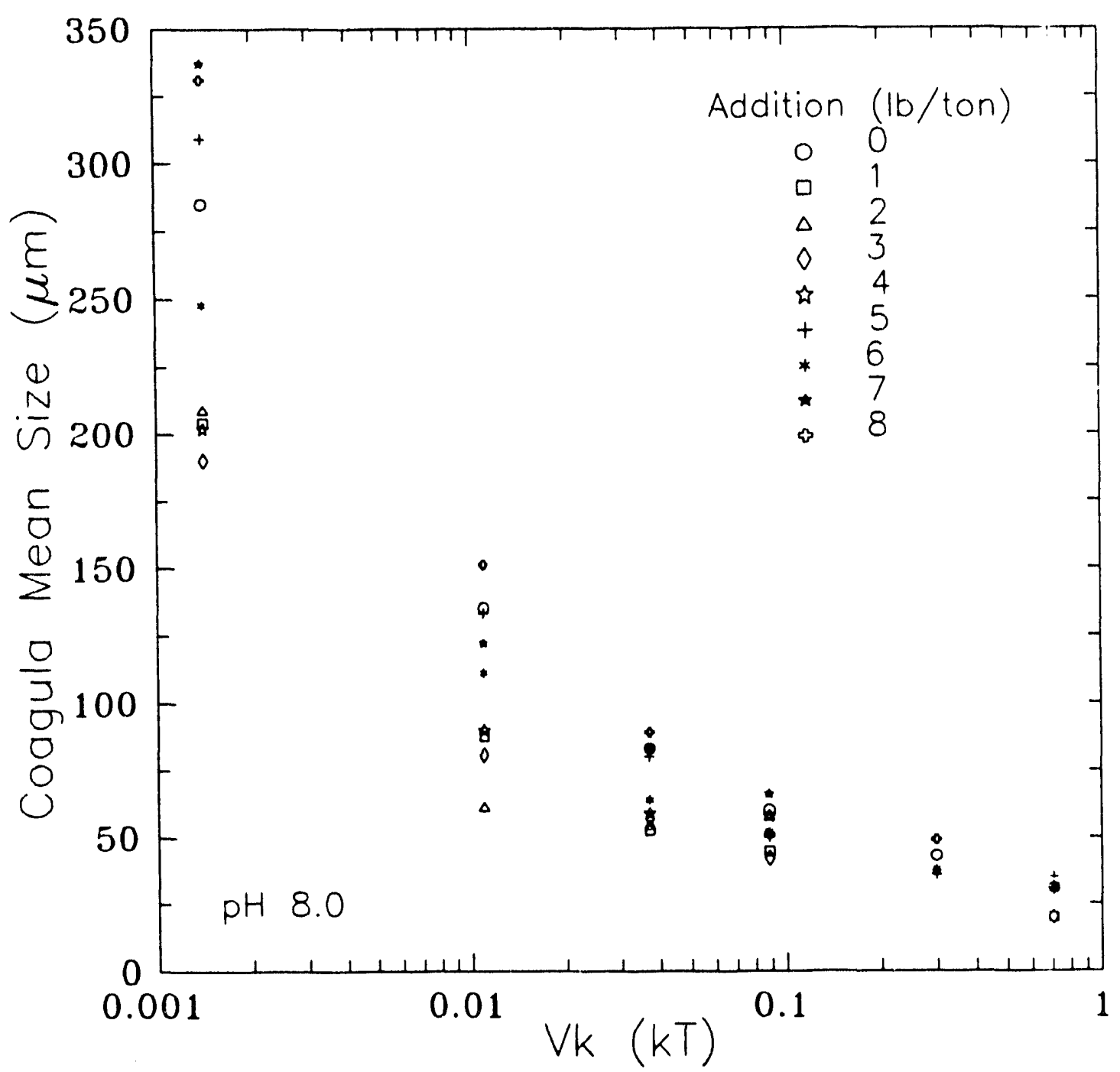

Figure 74. Effect of coal-oil addition and mixing intensity on mean coagula size for Elkhorn No. 3 seam coal. 


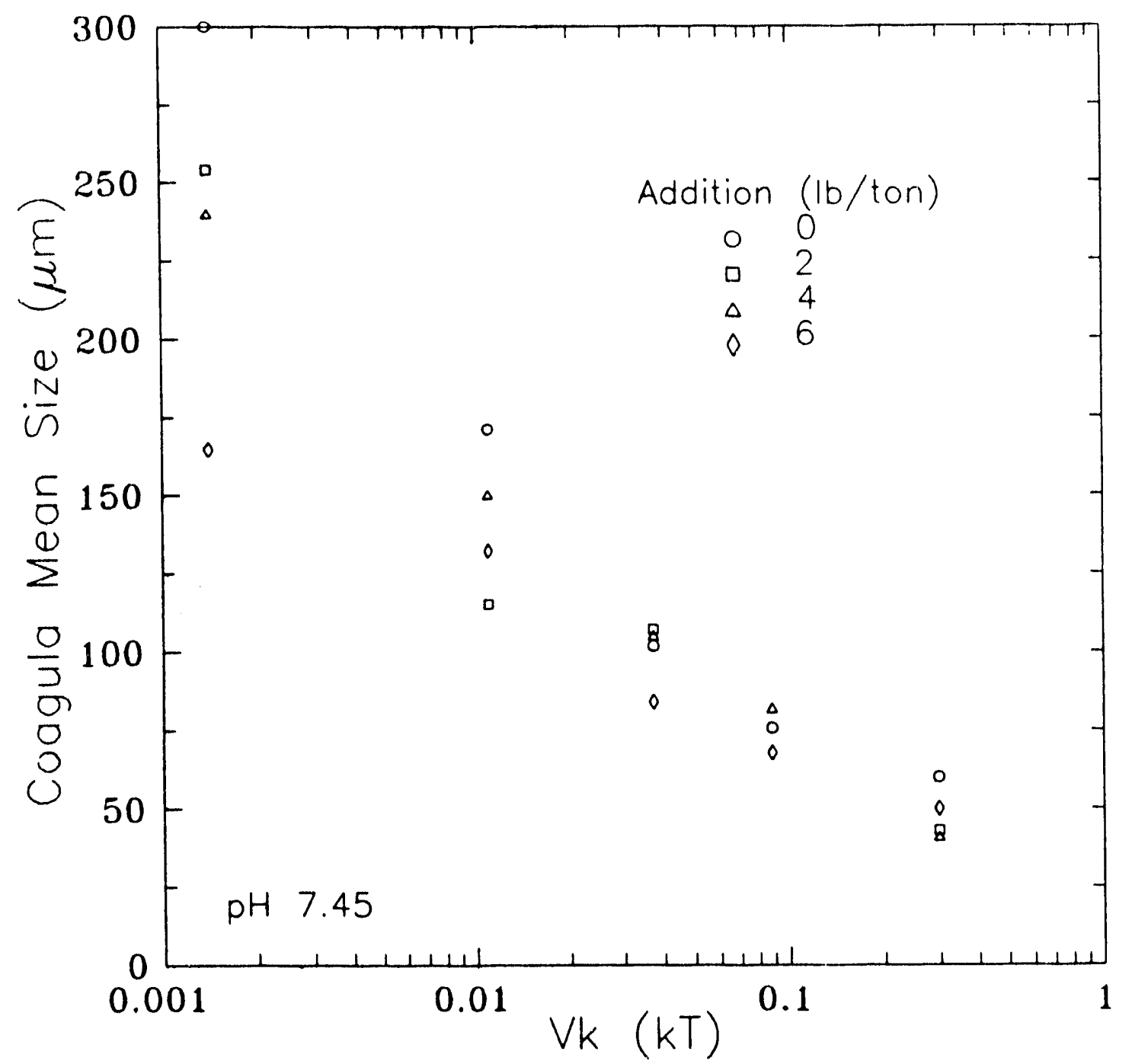

Figure 75. Effect of kerosene additions and mixing intensity on mean coagula size for Elkhorn No. 3 seam coal at pH 7.45. 


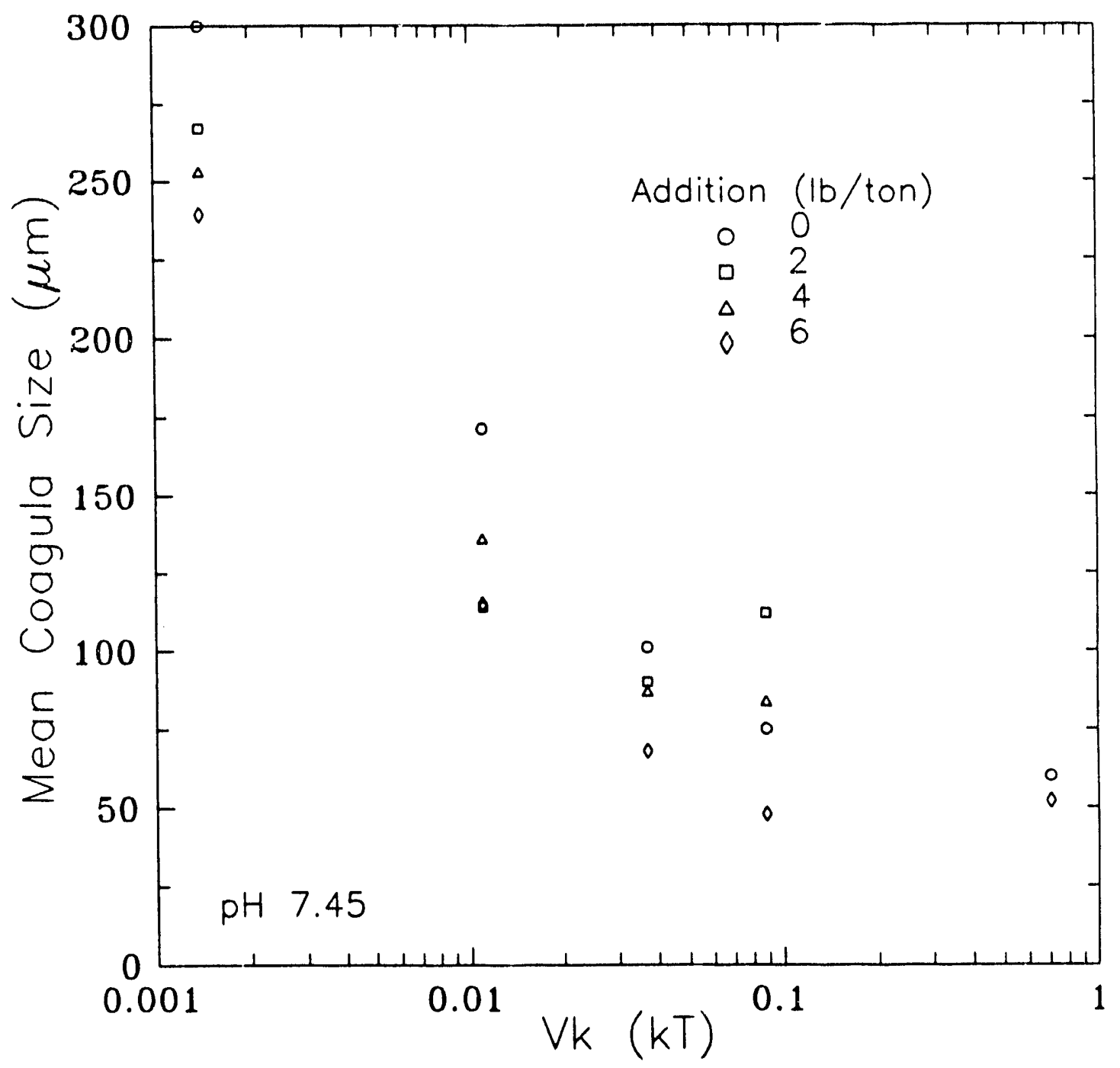

Figure 76. Effect of diesel addition and mixing intensity on mean coagula size for Elkhorn No. 3 seam coal at $\mathrm{pH} 7.45$. 
inhibit the growth of coagula (the only notable exception to this conclusion being coal oil at higher addition rates). Although a rational explanation for this behavior was not established, it may be possible that the oil additions simply resulted in the formation of denser coagula that were more tightly compacted.

The effect of hydrocarbon oil additions on settling rate were determined for Elkhorn No. 3 coal at pH 7. With the exception of using pH 7, the test materials were prepared in the same manner as above. The raw slurry was then placed in a 1-liter settling cylinder and distilled water in the amount needed was added. To provide an adequate concentration of counterions $10^{-3} \mathrm{M} \mathrm{KCl}$ was added and the $\mathrm{pH}$ adjusted. After the $\mathrm{pH}$ stabilized, the cylinder was rotated end-over-end three times, and the emulsion added. Then, the cylinder was turned end-over-end for 40 seconds to mix the system and promote droplet-particle contact. Thereafter, settling distances were noted, at one minute intervals, for at least 20 minutes. Results for kerosene, diesel and coal oil additions of 0$20 \mathrm{lbs} /$ ton are given in Figures 77, 78 and 79. Again, it will be noted that only coal oil has any significant (and positive) effect on coagula settling rates. Since earlier size estimation testwork had indicated that the size of the coagulum formed with each of these additives is quite similar, the only explanation for a notable difference in settling velocity is that there must be a difference in aggregate density. Although this may be due to an increase in the "compactness" of the coagula, coal oil's higher density $(1.03 \mathrm{~g} / \mathrm{ml})$ versus that for either kerosene $(0.746 \mathrm{~g} / \mathrm{ml})$ or diesel $(0.7 £ 5 \mathrm{~g} / \mathrm{ml})$ could be producing denser coagula. As can been seen from the graph of settling distance versus time, coal oil affects the packing characteristics of the coal. The zone of free settling is much shorter, and the slope changes drastically before the compression point. However, these graphs all indicate that small additions of hydrocarbon oils do not greatly affect the coagulation efficiency. 


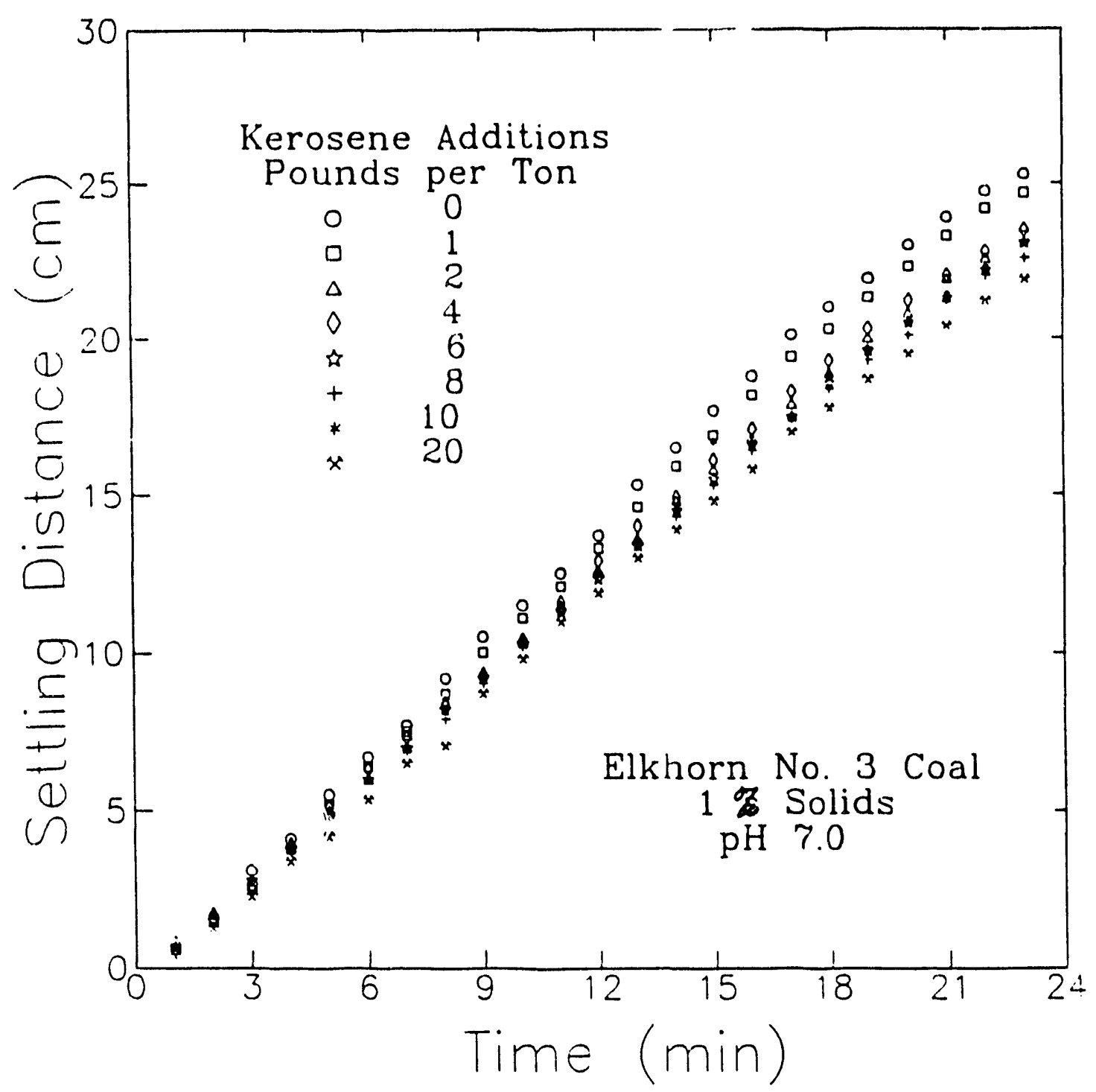

Figure 77. Effect of kerosene additions on coagula settling rates for Elkhorn No. 3 seam coal at $\mathrm{pH} 7$. 


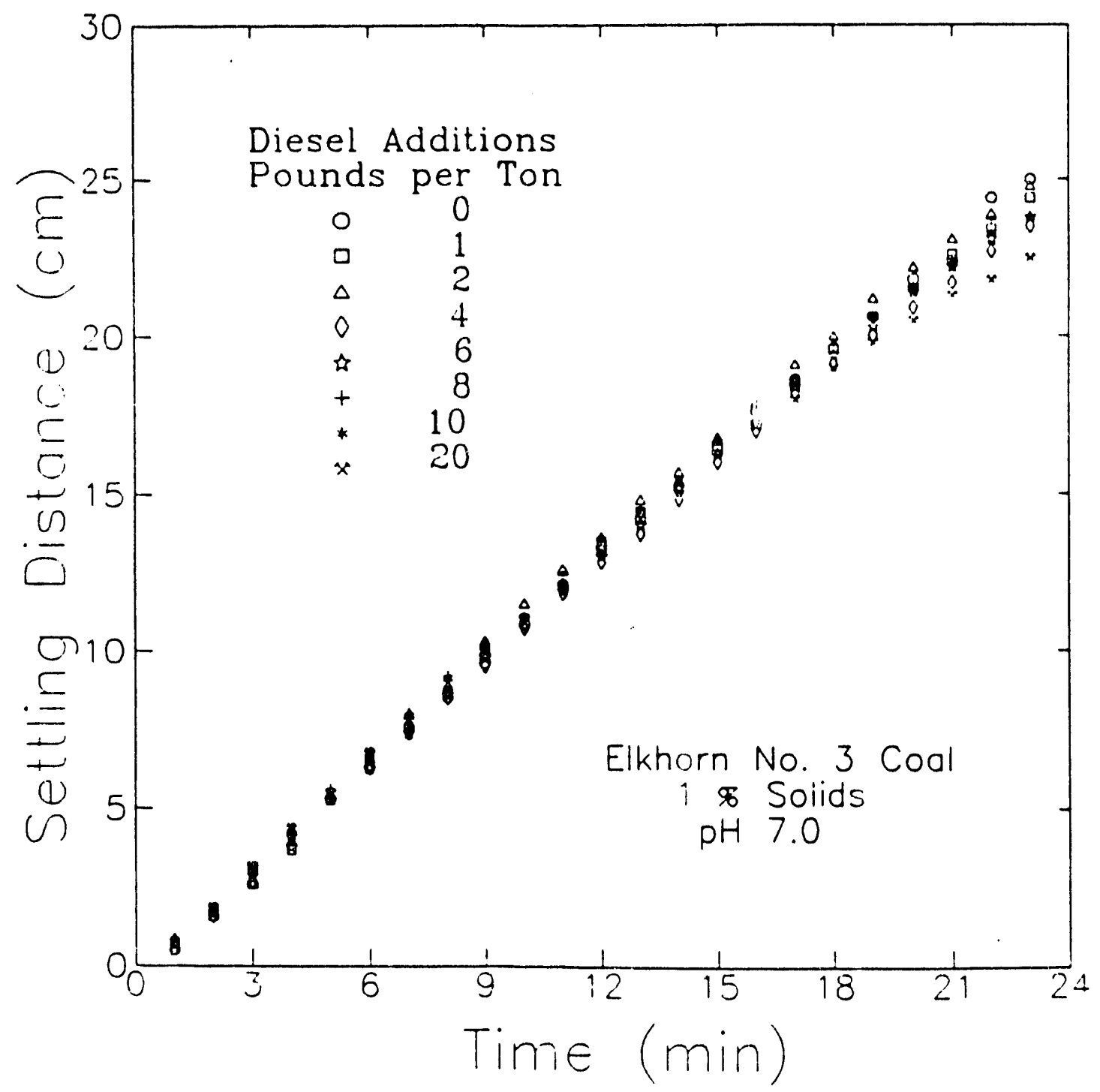

Figure 78. Effect of diesel additions on coagula settling rates for Elkhorn No. 3 seam coal at $\mathrm{pH} 7$. 


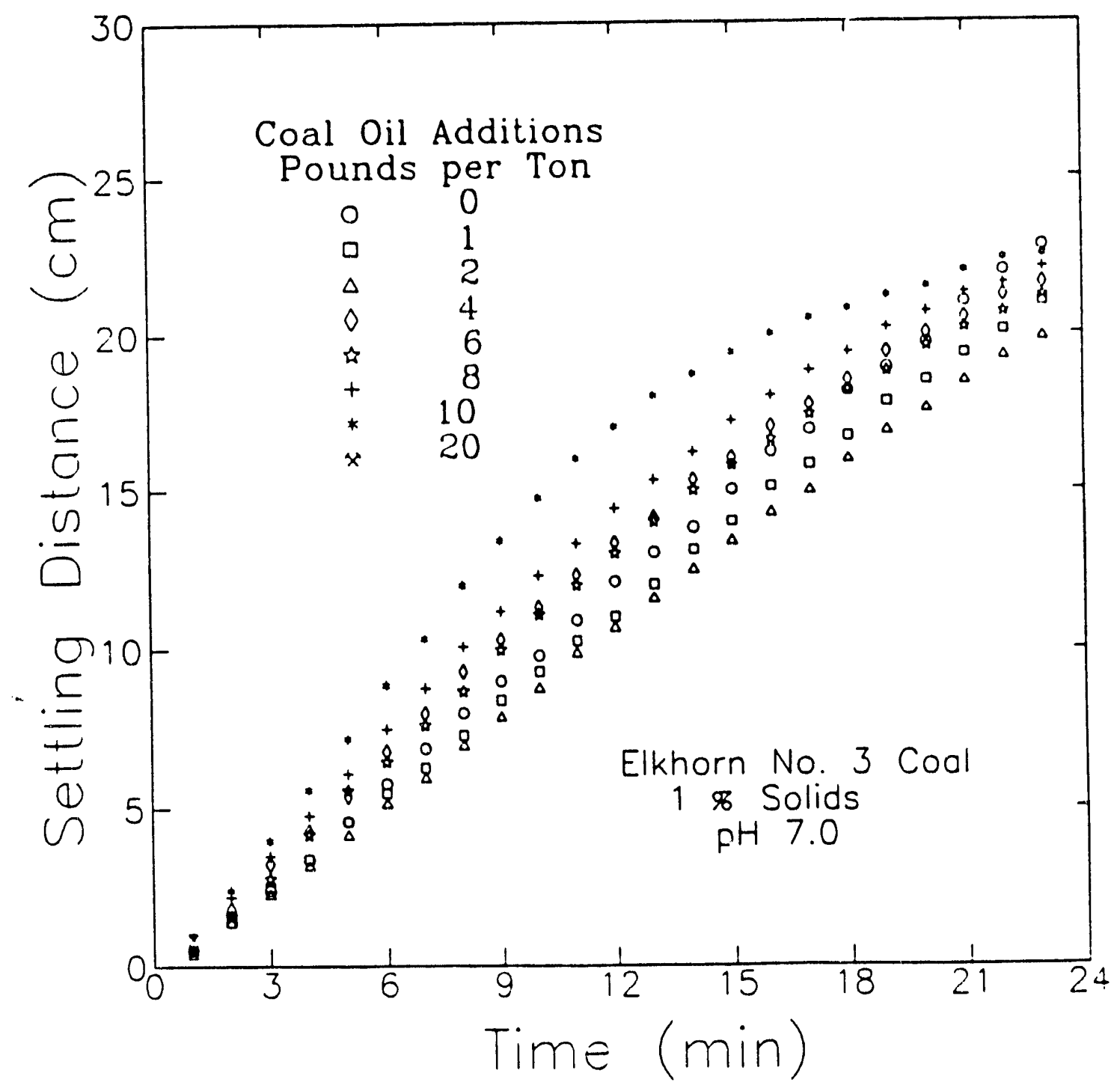

Figure 79. Effect of coal oil additions on coagula settling rates for Elkhorn No. 3 seam coal at $\mathbf{p H} 7$. 
Work was also conducted to determine the effect of hydrocarbon oil additions on a partially oxidized coal sample. The oxidized sample was prepared from a fresh Elkhorn No. 3 coal sample that had been ground to -200 mesh in a hammer mill and placed in an oven at $100^{\circ} \mathrm{C}$ for 24 hours. Thereafter it was ground in an attrition mill for 30 minutes to obtain a $3.5 \mu \mathrm{m}$ mean size. Settling tests were performed following the same procedure outlined for the unoxidized samples above. Since the effect of $\mathrm{pH}$ on oil and coal mixtures has not been discussed previously as part of this project, it was decided to perform the settling tests not only as a function of oil addition, but also of $\mathrm{pH}$. The results are shown in Figure 80. In all cases, the addition of kerosene has reduced settling velocities. Overall, the settling rates were greatest at $\mathrm{pH} 5.8$ and dropped off fairly uniformly on both sides of this optimum. Above pH 7.4 the supernatant became so turbid that the mudline could not be faithfully followed. At the lower end of the pH range the mudline was easily identified at $\mathrm{pH} 4.7$ and the plot could have been extended, but the downward trend had already been established. This result was unexpected. The i.e.p. for this coal is 3.5 and the zeta potential is negative above this value. Kerosene has a strongly negative zeta potential throughout the $\mathrm{pH}$ range, varying from -40 to -80 $\mathrm{mv}$, and one would expect greater affinity between the coal and kerosene in the lower $\mathrm{pH}$ range. This, in turn, should lead to a greater agglomerating influence in the lower $\mathrm{pH}$ range, i.e., larger aggregates and greater settling velocities, although this does not appear to have occurred here.

\section{Task 3.5 Separator Design and Construction}

It has been shown that ultrafine coal (hydrophobic) particles can be selectively coagulated and separated from dispersed hydrophilic particles using simple pH control. This selective hydrophobic coagulation (SHC) separation is achieved without the use of 


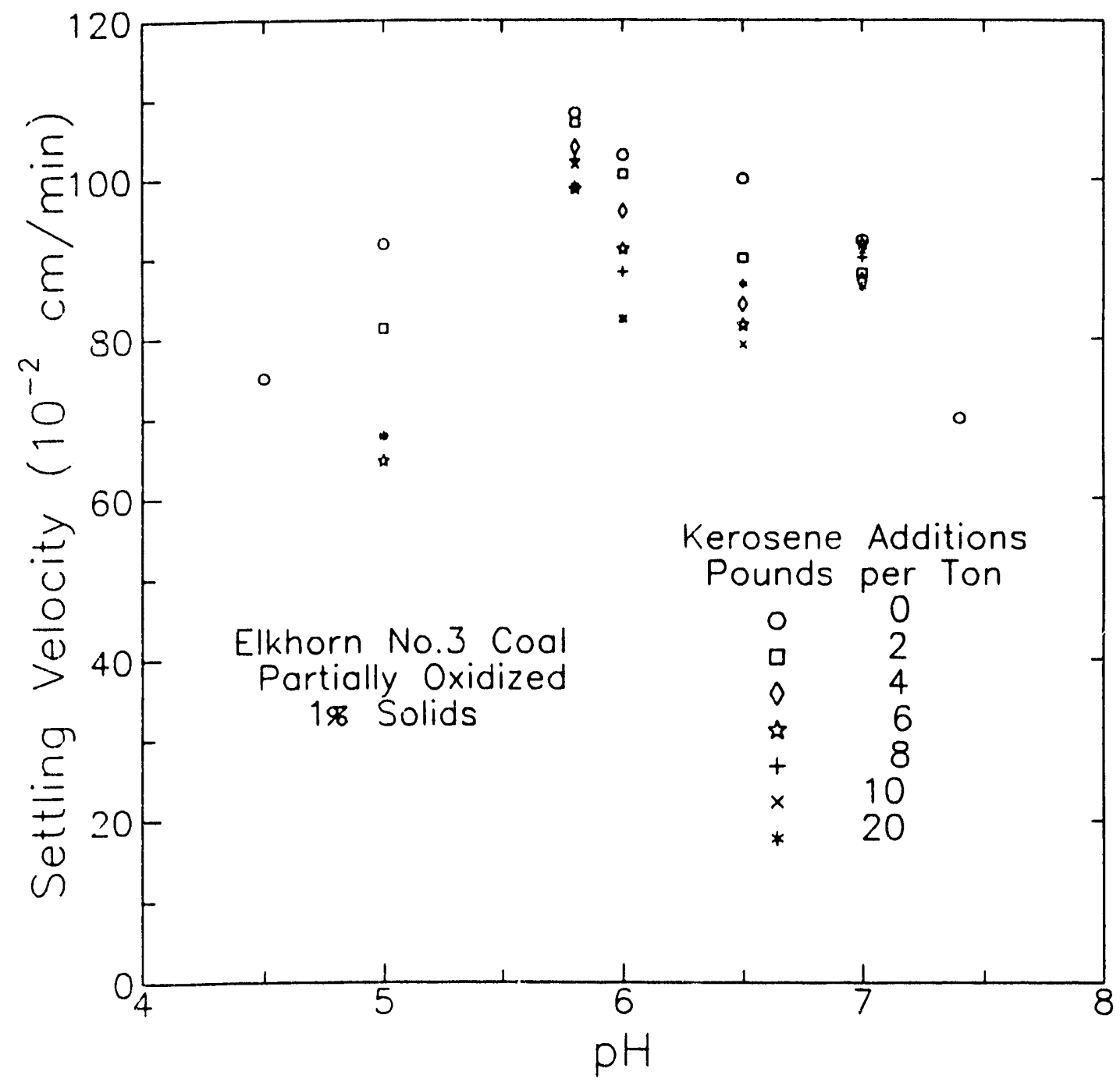

Figure 80. Effect of kerosene additions and $\mathrm{pH}$ on coagula settling rates for a partially oxidized sample of Elkhom No. 3 seam coal. 
agglomerants, flocculating agents, or dispersants. In a previous investigation ("Advanced Systems for Producing Superclean Coal, " DOE Final Report, DE-AC22-86PC91221, 1990), this technique has been proven capable of producing superclean (less than $2 \%$ ash, less than $1 \%$ sulfur) coal from a variety of eastern U.S. coal seams while maintaining high recoveries.

In selecting a technique for continuously separating the coal coagula from the dispersed mineral matter, one must consider the relatively weak binding strength that holds the coal coagula together. The ideal separator would be one that has maximum capacity per unit area while maintaining fairly quiescent conditions in the pulp. High capacity separators that are based on particle size and/or gravity, such as hydrocyclones, centrifuges, and screens, provide a turbulent environment that could potentially result in breakage of the coal coagula and a loss in coal recovery. At present, the most promising separator may be one that utilizes the difference between the settling velocity of the coal coagula and the mineral matter without the application of additional forces. Initial efforts to develop a continuous SHC process were reported in an earlier investigation (DE-AC22-86PC91221). In this earlier study, an elutriation column was used for separating coal coagula from dispersed mineral matter. Other researchers have shown that the elutriation technique can be successfully used for separating selectively flocculated material from the dispersed material (Appleton et al., 1975; Friend et al., 1973; Read, 1971). Although this device proved capable of reducing the ash content of a run-of-mine coal sample by as much as $62 \%$ in one cleaning stage, unacceptably long retention times were required to achieve high recoveries of the coagulated material. These long retention times were due to the low net settling velocity of the coagula which was caused by the slow terminal settling rate of the coagula and the upward velocity of elutriation water. This meant that the process would require a relatively large unit to 
treat respectable quantities of material. For this reason, separators consisting of a sedimentation tank and a rotating drum screen were developed and tested in this study.

A schematic of the sedimentation tank showing the dimensions and flow scheme is provided in Figure 81. The selectively coagulated feed slurry is fed into the center of the tank. The coal coagula settle to the bottom of the tank where the product is extracted from the cell at a flow rate substantially lower than that of the feed. As a result, the net upward flow in the cell carries the dispersed mineral matter out the top of the tank where it is collected as the tailings of the process. Several sedimentation tanks were constructed in order to perform staged cleaning tests.

A schematic of the rotating screen used for screening out the coagulated coal is shown in Figure 82. The screen separator consists of a 400-mesh nylon cloth wrapped around an 8-inch diameter drum that is continuously rotating at a very slow speed (less than $1 \mathrm{rpm})$. The drum is partially submerged in a pool of water. The feed slurry is fed into the interior of the drum through a static pipe. The dispersed mineral matter passes through the screen while the coal coagula are caught on the screen. As the screen rotates, the coagula are washed off the screen using a water spray into a stationary trough located inside the drum and then discharged out of the drum through a static tube. The dispersed mineral matter is collected through a tailings port located outside the pulp basin. The tailings port can be moved to adjust the pulp level inside the drum, thus providing a means for controlling retention time.

The performance of these two separation devices is discussed in the following section. 


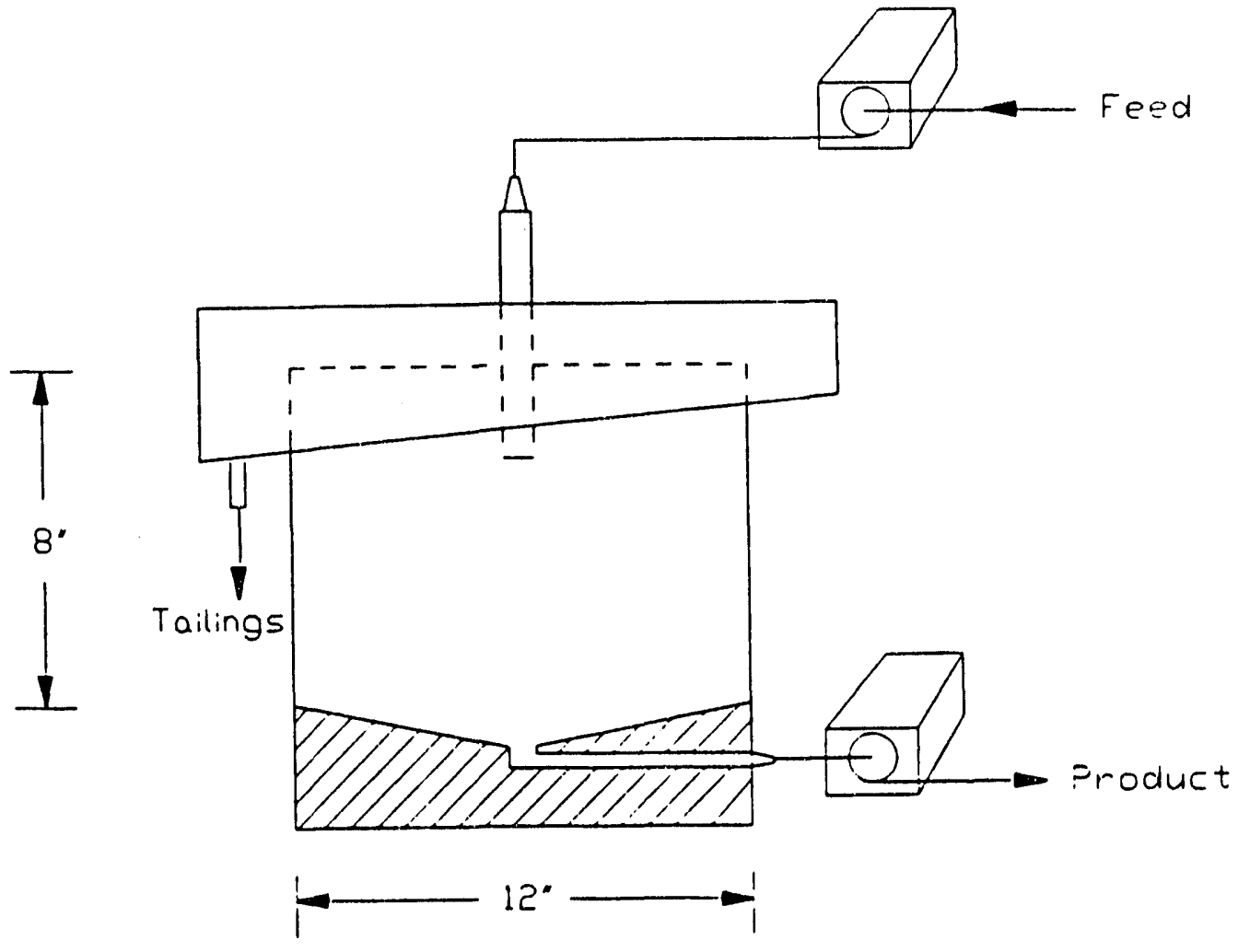

Figure 81. Schematic of the sedimentation tank to be used for separating coagulated coal particles from dispersed mineral matter. 
Wash Water $\longrightarrow \ldots$
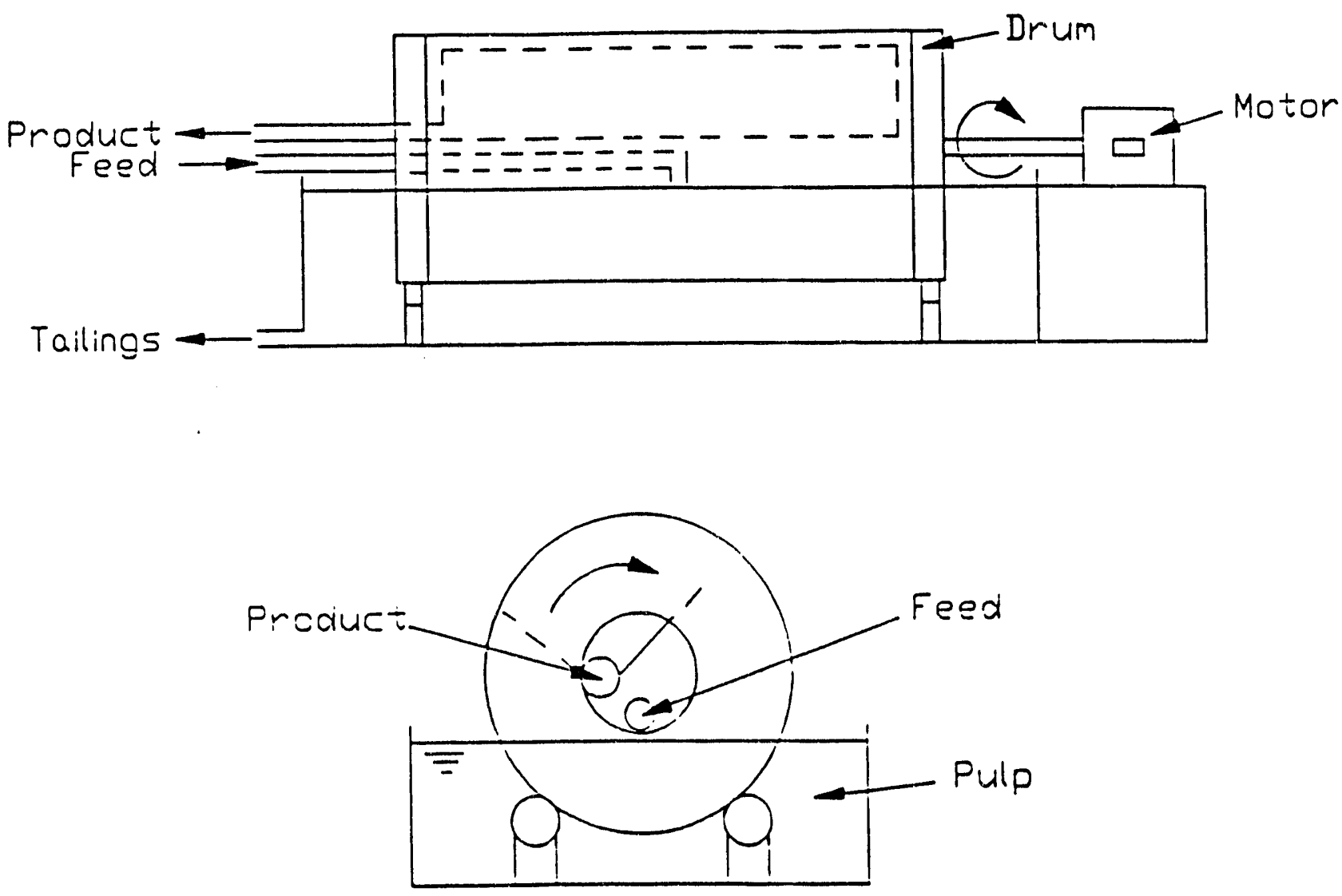

Figure 82. Schematic of the rotating screen to be used for separating coagulated coal particles from dispersed mineral matter. 


\section{Task 3.6 Bench-Scale Continuous Testing}

\section{Task 3.6.1 Low-Sulfur Coal Tests}

Exploratory tests were conducted on the sedimentation/elutriation tank and the rotating drum screen discussed above. An 8-inch diameter sedimentation tank was used in the exploratory tests instead of the proposed 11-inch diameter unit in order to run the maximum number of exploratory tests possible. The rotating drum has an 8 -inch diameter and utilized a 400 mesh screen cloth which was wrapped around the outer circumference of the drum.

Both devices were run continuously using micronized Elkhorn No. 3 coal seam samples having an ash content of approximately $22 \%$. The micronized feed slurries were obtained by grinding a -100 mesh coal sample in an attrition mill containing $1 / 8$-inch stainless steel grinding media for a period of 30 minutes. The mean volume particle size $\left(D_{s o}\right)$ was measured to be approximately $3.5 \mu \mathrm{m}$. The slurry was diluted to the desired solids concentrations using tap water, while the $\mathrm{pH}$ was maintained at a value of approximately 7.3. Samples were taken of the feed, product and tailing streams after the device had been operating for a period equal to 1-2 retention times. Each sample was filtered, dried, weighed, and analyzed for ash content.

Initial experinents using the sedimentation tank were aimed at determining the maximum throughput possible for the given geometric configuration. This was accomplished by varying both the feed solids concentration (\% by weight) and feed rate. The effect of solid concentration on separation performance was obtained by setting the feed rate, feed point, and mudline level at $65 \mathrm{ml} / \mathrm{min}, 3.18 \mathrm{~cm}$, and $13 \mathrm{~cm}$, respectively. The mudline is formed as a result of the concentration of settled aggregates or coagula. The test results shown in Table $\mathrm{IX}$ indicate that increasing the solids content improves 
Table IX. Tabulated results illustrating the effect of feed solids content on the separation performance of a continuous sedimentation tank (lhickener).

\begin{tabular}{|c|c|c|c|c|}
\hline $\begin{array}{c}\text { Feed Solids } \\
\text { Content } \\
(\%)\end{array}$ & $\begin{array}{c}\text { Product } \\
\text { Ash } \\
(\%)\end{array}$ & $\begin{array}{c}\text { Tailings } \\
\text { Ash } \\
(\%)\end{array}$ & $\begin{array}{c}\text { Coal } \\
\text { Recovery } \\
(\%)\end{array}$ & $\begin{array}{c}\text { Mass Flow } \\
\text { Rate/Area } \\
\left(\mathrm{g} / \mathrm{min} / \mathrm{cm}^{2}\right)\end{array}$ \\
\hline 2.0 & 8.88 & 65.9 & 91.2 & 0.0031 \\
\hline 3.0 & 11.8 & 76.9 & 96.1 & 0.0044 \\
\hline 5.0 & 21.9 & 79.5 & 99.6 & 0.0073 \\
\hline
\end{tabular}

combustible recovery, which is probably due to an increase in particle number population. However, increasing the solids content from 2 to $5 \%$ resulted in an increase in the product ash content from $8.88 \%$ to $21.9 \%$. The increase in product ash content was most likely due to an increase in entrapment of gangue particles in the coal coagula.

Feed rate to the sedimentation tank was varied over the range of 50 to $100 \mathrm{ml} / \mathrm{min}$ while maintaining the solids concentration, feed point, and mudline level at $3 \%, 3.18 \mathrm{~cm}$, and $13 \mathrm{~cm}$, respectively. Increasing the feed rate over the range studied resulted in a slight decrease in combustible recovery as shown in Table X. This may be explained by the fact that increasing the feed rate increases the upflow velocity in the sedimentation tank, thus resulting in the smaller coal coagula reporting to the tailings overflow launder. As a result, the ash content in the tailings decreases as illustrated in Table X. The upper limit on feed rate is determined by whether or not the mud level can be controlled. In a properly operated sedimentation tank, the coagula or agglomerates settle out before reaching the inside walls. As a result, the mudline is clear and controllable by adjusting the rate of the underflow discharge. If the coagula has not settled out prior to reaching the inside wall, the mudline is unclear and difficult to control. In the exploratory tests, maximum feed rate was found to be approximately $100 \mathrm{ml} / \mathrm{min}$. This feed rate 
Table X. Tabulated results illustrating the effect of feed rate on the separation performance of a continuous sedimentation tank (thickener).

\begin{tabular}{|c|c|c|c|c|}
\hline $\begin{array}{c}\text { Feed } \\
\text { Rate } \\
(\mathrm{ml} / \mathrm{min})\end{array}$ & $\begin{array}{c}\text { Product } \\
\text { Ash } \\
(\%)\end{array}$ & $\begin{array}{c}\text { Tailings } \\
\text { Ash } \\
(\%)\end{array}$ & $\begin{array}{c}\text { Coal } \\
\text { Recovery } \\
(\%)\end{array}$ & $\begin{array}{c}\text { Mass Flow } \\
\text { Rate/Area } \\
\left(\mathrm{g} / \mathrm{min} / \mathrm{cm}^{2}\right)\end{array}$ \\
\hline 50 & 11.8 & 76.9 & 96.0 & 0.0044 \\
\hline 65 & 10.9 & 71.0 & 93.5 & 0.0059 \\
\hline 85 & 13.6 & 63.0 & 92.6 & 0.0078 \\
\hline 100 & 12.7 & 60.8 & 91.9 & 0.0089 \\
\hline
\end{tabular}

corresponds to approximately $0.3 \mathrm{lb} / \mathrm{hr}$. A larger 11 -inch diameter tank will be tested in the future to obtain our goal of $1-2 \mathrm{lb} / \mathrm{hr}$.

The addition of wash water under the mudline was also tested for two reasons: i) tc replace water in the concentrate containing entrained mineral matter with clean water and ii) to provide a higher upward velocity to help wash the heavier mineral matter out with the overflow. The wash water addition rates were varied over a range of 10 to 30 $\mathrm{ml} / \mathrm{min}$ using a feed rate and solids concentration of $50 \mathrm{ml} / \mathrm{min}$ and $3 \%$, respectively. As shown in Table XI, the results indicate that the addition of wash water below the mudline improves the product ash content slightly while recovery decreases substantially. Based on these results, the addition of wash water below the mudline is not recommended, due to a decrease in separation efficiency (i.e., from 53.7 to $35.5 \%$ ).

The exploratory tests for the rotating drum screen involved studying the feed rate and solid concentrations over the ranges of 40 to 100 and 3 to $5 \%$ by weight, respectively. As shown in Table XII, the product ash content increased slightly from 11.6 to $12.5 \%$ when the solids content was increased from 3 to $5 \%$. Over the same range, combustible recovery sharply increased from 13.8 to $63.8 \%$. At a solids content of $5 \%$, an increase in feed rate from 50 to $75 \mathrm{ml} / \mathrm{min}$ resulted in an increase in product 
Table XI. Tabulated results illustrating the effect of wash water rate on the separation performance of a continuous sedimentation tank.

\begin{tabular}{|c|c|c|c|c|}
\hline $\begin{array}{c}\text { Wash Water } \\
\text { Rate } \\
(\mathrm{ml} / \mathrm{min})\end{array}$ & $\begin{array}{c}\text { Product } \\
\text { Ash } \\
(\%)\end{array}$ & $\begin{array}{c}\text { Tailings } \\
\text { Ash } \\
(\%)\end{array}$ & $\begin{array}{c}\text { Coal } \\
\text { Recovery } \\
(\%)\end{array}$ & $\begin{array}{c}\text { Mass Flow } \\
\text { Rate/Area } \\
\left(\mathrm{g} / \mathrm{min} / \mathrm{cm}^{2}\right)\end{array}$ \\
\hline \hline 10 & 11.1 & 79.3 & 95.8 & 0.0058 \\
\hline 20 & 11.1 & 69.7 & 92.9 & 0.0058 \\
\hline 30 & 10.4 & 59.2 & 87.8 & 0.0058 \\
\hline
\end{tabular}

Table XII. Tabulated results illustrating the effect of feed rate and feed solids content on the separation performance of a continuously rotating drum screen.

\begin{tabular}{|c|c|c|c|c|c|}
\hline $\begin{array}{c}\text { Feed Rate } \\
(\mathrm{ml} / \mathrm{min})\end{array}$ & $\begin{array}{c}\text { Feed Solids } \\
\text { Content } \\
(\%)\end{array}$ & $\begin{array}{c}\text { Product } \\
\text { Ash } \\
(\%)\end{array}$ & $\begin{array}{c}\text { Tailings } \\
\text { Ash } \\
(\%)\end{array}$ & $\begin{array}{c}\text { Coal } \\
\text { Recovery } \\
(\%)\end{array}$ & $\begin{array}{c}\text { Mass Flow } \\
\text { Rate/Area } \\
\left(\mathrm{g} / \mathrm{min} / \mathrm{cm}^{2}\right)\end{array}$ \\
\hline \hline 50 & 3 & 11.6 & 22.6 & 13.8 & 0.0014 \\
\hline 75 & 3 & 13.2 & 22.8 & 10.8 & 0.0021 \\
\hline 100 & 3 & 15.2 & 22.2 & 0.36 & 0.0028 \\
\hline & & & & & \\
\hline 40 & 4 & 13.0 & 36.8 & 65.5 & 0.0013 \\
\hline 60 & 4 & 19.0 & 27.5 & 56.0 & 0.0019 \\
\hline 80 & 4 & 19.8 & 27.8 & 62.2 & 0.0030 \\
\hline & & & & & \\
\hline 50 & 5 & 12.5 & 35.9 & 63.1 & 0.0024 \\
\hline 75 & 5 & 20.2 & 26.1 & 56.5 & 0.0035 \\
\hline
\end{tabular}

ash content from 12.5 to $20.2 \%$, while combustible recovery decreased from 35.9 to $26.1 \%$. 
The results obtained with the drum screen may be explained by the fact that the overall strength of the coagula is very small as discussed in Task 3.3. An increase in the feed rate results in an increase in the force pushing the coagula through the screen. In response to the increased shearing force, the coagula tend to deform and/or break and pass through the screen. This leads to an overall decrease in the recovery of the coagula. In contrast, a larger solids concentration tends to improve the recovery of coal coagula. This behavior is due to an increased particle population density which increases the coagula size (Task 2.3) and "blinding" due to a larger solids load on the screen. As indicated in Table XII, both these phenomena result in an increase in coagula recovery. Increased product ash contents under these conditions were a result of increased entrapment in the solids bed on the screen. Thus, recovery of the coal coagula is improved while product ash increases.

A comparison of the results obtained from the two separators indicates that the sedimentation tank yields lower product ash content and substantially higher combustible recovery values at larger mass flow rates. At a mass feed rate per cross-sectional area of $0.0031 \mathrm{~g} / \mathrm{min} / \mathrm{cm}^{2}$, the sedimentation tank gave a product ash content and a combustible recovery value of 8.88 and $91.2 \%$, respectively. This corresponds to a separation efficiency of $60.6 \%$. In comparison, the rotating drum screen yielded a product ash content of $12.5 \%$ and a combustible recovery value of $63.1 \%$ at a lower mass feed rate of $0.0024 \mathrm{~g} / \mathrm{min} / \mathrm{cm}^{2}$. As a result, the continuous sedimentation tank was chosen as the device to be used in future testwork involving reagent addition and process optimization studies.

This work was conducted on a low-sulfur Elkhorn No. 3 seam coal seam $(22.5 \%$ feed ash) using the 11-inch diameter sedimentation tank separator described in Task 3.5. Experiments were conducted to determine the effects of feed rate, solids concentration, 
$\mathrm{pH}$, initial particle size, and the addition of chemical additives (e.g., chelating agents and dispersants) on separator performance. The primary goal of this exercise was to demonstrate the continuous operation of the bench-scale $S H C$ process at a 1-2 lb/hr of feed rate.

The continuous SHC tests were conducted using samples that were ground to 2.8 $\mu \mathrm{m}$ in a stirred ball mill. Unless otherwise indicated, the solids concentration for each test was maintained at $3 \%$ solids by weight using tap water. The $\mathrm{pH}$ of the coal slurry was adjusted to the desired value (usually 7.5 ) through the addition of sodium hydroxide. The standard operating conditions used throughout the test program were:

$\begin{array}{ll}\text { Feed Rate } & =250 \mathrm{ml} / \mathrm{min} \\ \text { Feed Solids } & =3 \% \\ \text { Feed Point } & =11 / \mathrm{in} \\ \text { Mudline } & =41 / 2 \mathrm{in} \\ \mathrm{pH} & =7.5\end{array}$

Both the location of the feed point and mudline were measured from the top of the sedimentation tank. In some experiments, a chelating agent (EDTA) was also added to the suspension at a concentration of $0.3 \mathrm{~g} /$ liter to complex the multi-valent ions which are believed to suppress the selectivity of the process by inducing coagulation of the mineral matter.

In order to determine the amount of time required for the process to reach steadystate, a preliminary shakedown test was conducted in which samples of the product and reject streams were taken as a function of time and analyzed for ash content. As shown in Figure 83, the process required approximately 80 minutes before the product ash content reached a constant value. Based on a cell volume of 10 !iters, the time required 


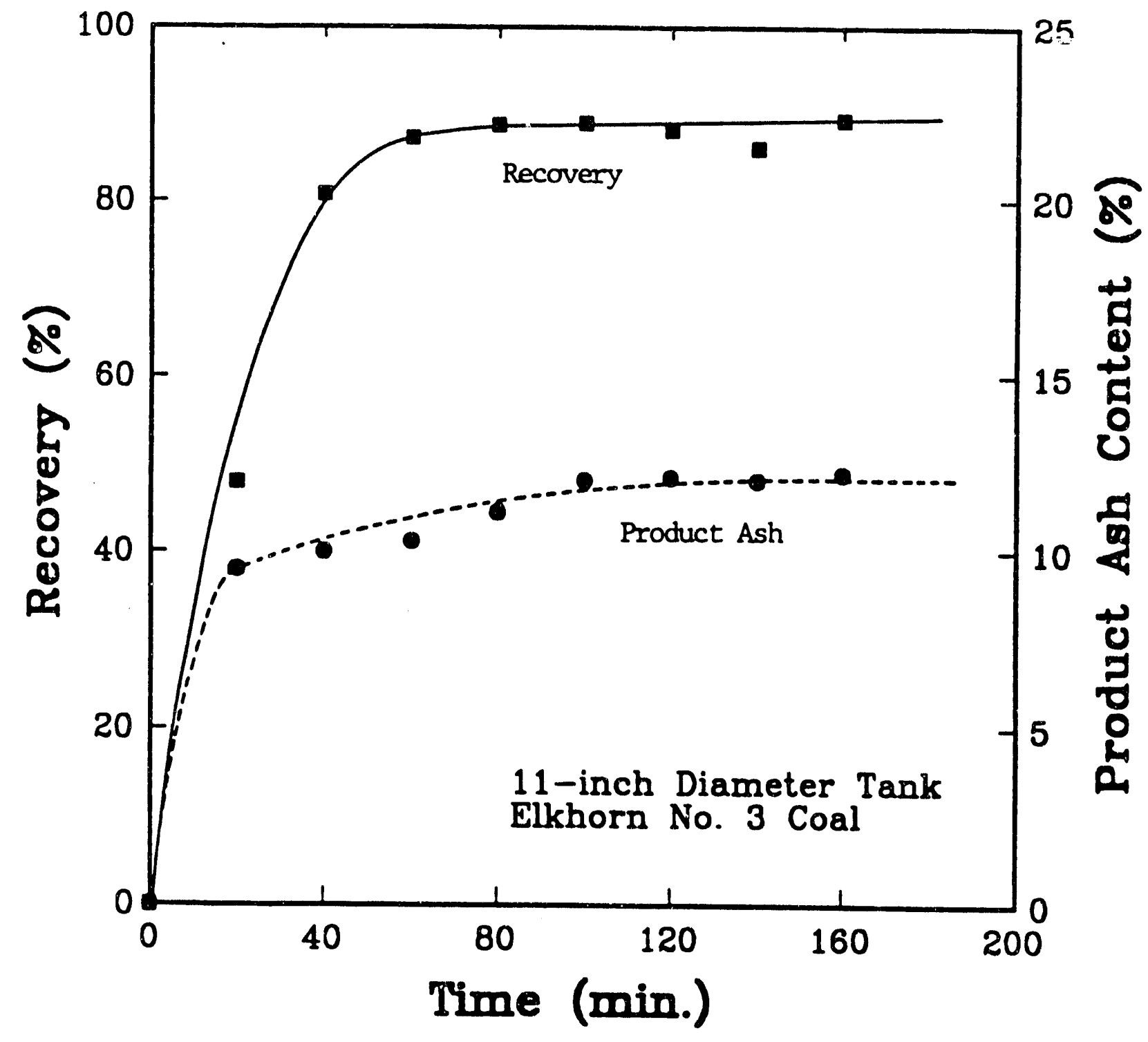

Figure 83. Coal recovery and product ash content vs. retention time in the continuous, bench-scale treatment of an Elkhorn No. 3 seam coal. 
to reach steady-state was found to be equal to approximately twice the slurry retention time (i.e., cell volume/feed rate). Therefore, the remaining tests were conducted for a minimum of two retention times before representative samples of the product, tailings, and feed streams were collected. Each sample collected by this procedure was analyzed for solids concentration and ash content. Steady-state operation was confirmed by backcalculating the feed ash content using mass flow rates of the product, tailing, and feed streams and comparing this result with that determined for the head sample. Coal recovery and separation efficiency values were also calculated for each test.

The first operating parameter examined in this study was volumetric feed flow rate. A range of feed rates from 200 to $400 \mathrm{ml} / \mathrm{min}$, i.e., a mass feed rate of 0.8 to 1.6 $\mathrm{lb} / \mathrm{hr}$, was examined in this test series. Unlike the batch tests, only a single stage of cleaning was employed in the continuous test program. As shown in Table XIII, the maximum separation efficiency of $49.0 \%$ (and the minimum product ash value of $10.9 \%$ ) was obtained at a feed flow rate of $350 \mathrm{ml} / \mathrm{min}(1.4 \mathrm{lb} / \mathrm{hr})$. The corresponding combustible recovery obtained at this throughput was $84.7 \%$. A further increase in feed rate caused a substantial drop in combustible recovery due to excessive mixing within the separator. Feed rates lower than $350 \mathrm{ml} / \mathrm{min}$ gave higher combustible recoveries but also resulted in higher product ash contents. The increase in ash content with decreasing feed rate can be attributed to the accompanying drop in the upward flow velocity in the separator. In order to achieve low ash levels, this velocity must be maintained at a level sufficient to wash the particles of mineral matter out the top of the sedimentation separator.

The effect of variations in the feed solids content has also been studied. Solids concentrations of $2.0 \%$ and $3.0 \%$ by weight were examined over a range of feed rates. As shown in Figure 84, the separation efficiency for both solids concentrations reached a 
Table XIII. Results illustrating the effect of feed rate on the separation performance of the SHC process using an 11 -inch diameter continuous sedimentation tank.

\begin{tabular}{|c|c|c|c|c|c|}
\hline $\begin{array}{c}\text { Feed Flow } \\
\text { Rate }\end{array}$ & $\begin{array}{c}\text { Feed Mass } \\
\text { Rate }\end{array}$ & $\begin{array}{c}\text { Product } \\
\text { Ash } \\
\text { Content } \\
(\mathrm{ml} / \mathrm{min})\end{array}$ & $\begin{array}{c}\text { Tailing Ash } \\
\text { Content } \\
(\%)\end{array}$ & $\begin{array}{c}\text { Combustible } \\
\text { Recovery }\end{array}$ & $\begin{array}{c}\text { Separation } \\
\text { Efficiency }\end{array}$ \\
\hline \hline 200 & 0.8 & 21.4 & 58.3 & 98.3 & $(\%)$ \\
\hline 250 & 1.0 & 13.3 & 62.4 & 90.9 & 4.34 \\
\hline 300 & 1.2 & 12.1 & 63.2 & 90.3 & 47.5 \\
\hline 350 & 1.4 & 10.9 & 54.9 & 84.7 & 49.0 \\
\hline 400 & 1.6 & 11.2 & 38.1 & 66.4 & 37.5 \\
\hline
\end{tabular}

plateau at a volumetric feed flow rate of approximately $250 \mathrm{ml} / \mathrm{min}$. However, the highest overall separation efficiencies were obtained at the lower solids concentration of $2.0 \%$. The decrease in selectivity with increasing particle concentration is probably due to an increase in the entrapment of ultrafine mineral matter in the coal coagula.

Continuous coagulation tests were also conducted over a pH range from 7.0 to 8.3 in order to determine the effect of $\mathrm{pH}$ on the selectivity of the continuous process. The results of these experiments are given in Table XIV. As shown, the highest separation efficiency, $42.9 \%$, was obtained at $\mathrm{pH} 7.5$, with corresponding product ash content and recovery values of $13.3 \%$ and $90.9 \%$. The lowest product ash content after a single cleaning stage was obtained at $\mathrm{pH} 8.0$, but the combustible recovery at this $\mathrm{pH}$ was only $67 \%$, resulting in a lower separation efficiency of $38.8 \%$. These results indicate that lower $\mathrm{pH}$ values give higher combustible recoveries but also higher product ash contents. The increased ash content at the lower $\mathrm{pH}$ values may be due to enhanced heterocoagulation of coal and mineral matter or to the increased entrapment of fine mineral matter in the larger coal coagula formed at this pH. Low recoveries and high 


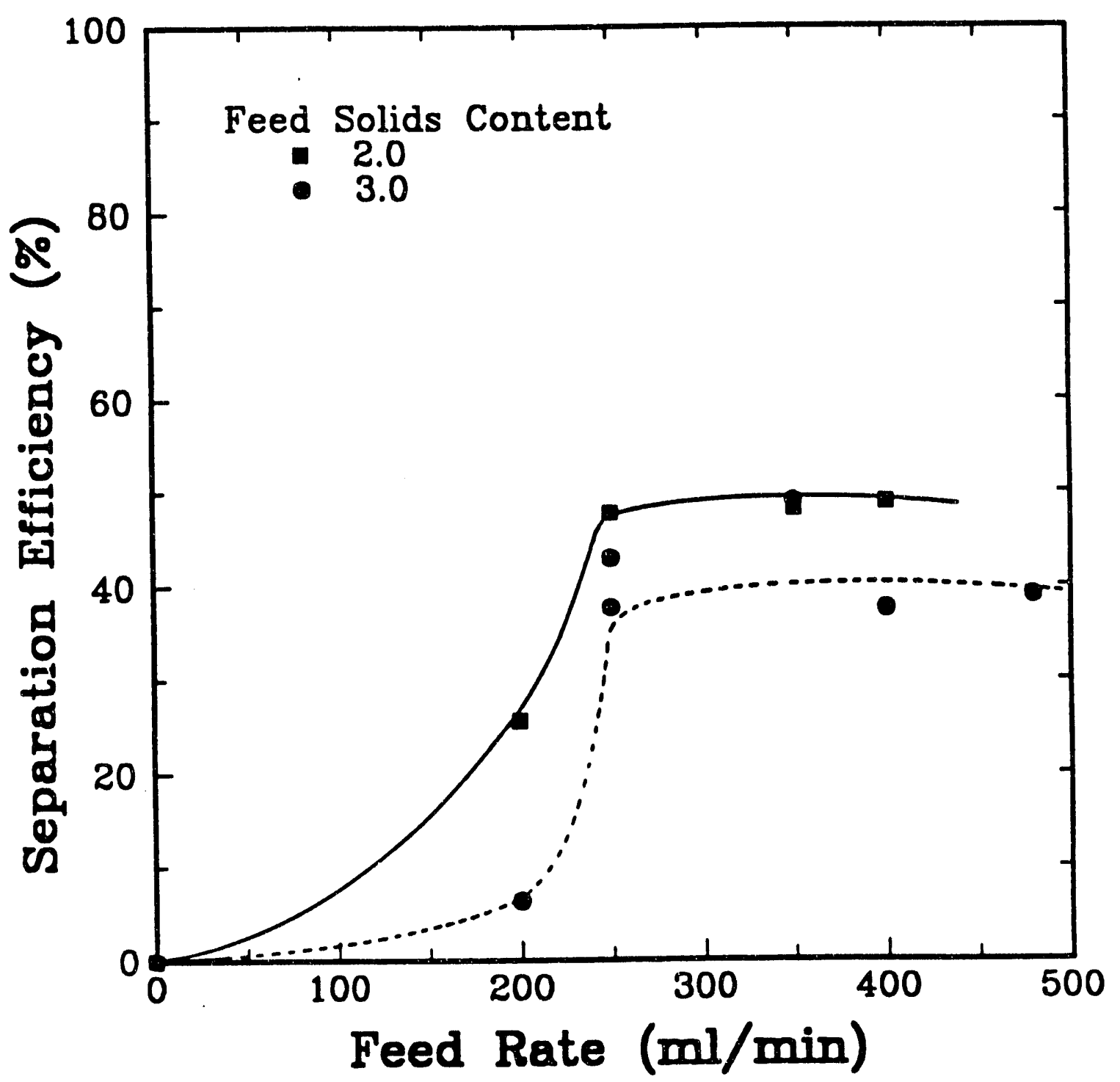

Figure 84. The effect of solids concentration on the separation performance of the continuous SHC unit when processing an Elkhorn No. 3 seam coal. 
product ash values were obtained at $\mathrm{pH}$ values greater than 8.0 due to the increased dispersion of the coal particles. Thus, the optimum operating $\mathrm{pH}$ for this particular coal lies between 7.5 and 8.0 .

Table XIV. Results illustrating the effect of $\mathrm{pH}$ on the efficient separation of selectively coagulated Elkhorn No. 3 coal from its associated mineral matter using an 11inch diameter continuous sedimentation tank.

\begin{tabular}{|c|c|c|c|c|}
\hline $\mathrm{pH}$ & $\begin{array}{c}\text { Product Ash } \\
\text { Content } \\
(\%)\end{array}$ & $\begin{array}{c}\text { Tailing Ash } \\
\text { Content } \\
(\%)\end{array}$ & $\begin{array}{c}\text { Combustible } \\
\text { Recovery } \\
(\%)\end{array}$ & $\begin{array}{c}\text { Separation } \\
\text { Efficiency } \\
(\%)\end{array}$ \\
\hline 7.0 & 14.9 & 74.6 & 96.0 & 40.0 \\
\hline 7.5 & 13.3 & 62.4 & 90.9 & 42.9 \\
\hline 8.0 & 10.9 & 38.7 & 67.0 & 38.8 \\
\hline 8.3 & 13.8 & 24.4 & 20.2 & 9.10 \\
\hline
\end{tabular}

The effect of initial feed particle size on the coagulation behavior of coal samples obtained from the Elkhorn No. 3 coal seam was also examined. In these tests, samples of the feed coal were ground to mean particle diameters of 7.5, 5.2, 4.1, and $2.8 \mu \mathrm{m}$ using a stirred ball mill. As shown in Table XV, the test data obtained with this coal indicate that a decrease in particle size results in a higher overall separation efficiency and a lower product ash content. This improvement may be caused by the increased liberation of mineral matter, a decrease in the settling velocity of the finely pulverized particles of mineral matter, and/or the formation of larger coagula as described previously in Task 2.4. Unfortunately, the effects of initial particle size on combustible recovery are unclear in this series of test runs.

In an attempt to improve the selectivity of the $S H C$ process, the individual and combined effects of the addition of dispersant (sodium hexametaphosphate) and chelating agent (EDTA) were also investigated. The dispersant was used to increase the 
Table XV. Results illustrating the effect of particle size on the separation performance of the SHC process using an 11-inch diameter continuous sedimentation tank.

\begin{tabular}{|c|c|c|c|c|}
\hline $\begin{array}{c}\mathrm{D}_{s 0} \\
(\mu \mathrm{m})\end{array}$ & $\begin{array}{c}\text { Product Ash } \\
\text { Content } \\
(\%)\end{array}$ & $\begin{array}{c}\text { Tailing Ash } \\
\text { Content } \\
(\%)\end{array}$ & $\begin{array}{c}\text { Combustible } \\
\text { Recovery } \\
(\%)\end{array}$ & $\begin{array}{c}\text { Separation } \\
\text { Efficiency } \\
(\%)\end{array}$ \\
\hline 2.8 & 7.11 & 50.0 & 76.9 & 56.6 \\
\hline 4.1 & 9.35 & 44.4 & 73.1 & 47.1 \\
\hline 5.2 & 11.3 & 49.9 & 81.2 & 45.7 \\
\hline 7.5 & 13.5 & 41.5 & 75.8 & 35.1 \\
\hline
\end{tabular}

electrostatic charge on the surface of the mineral matter, thereby retarding the coagulation of mineral matter. The chelating agent was added in an attempt to complex multi-valent ions that have been shown to adversely impact the selectivity of the process.

In order to establish a baseline result, neither EDTA or sodium hexametaphosphate (HMP) was added in the first continuous test run. As shown in Table XVI, the baseline tests resulted in a very high combustible recovery of $99.5 \%$ and a corresponding product ash value almost equal to that of the feed material. The addition of $4.0 \mathrm{lb} /$ ton of the dispersant reduced the product ash content was reduced from $22.4 \%$ to $14.8 \%$ with very little loss in combustible recovery. By comparison, the addition of the chelating agent alone reduced the product ash content to $13.3 \%$ at a somewhat lower recovery of $90.9 \%$. However, the best results were obtained by adding both a chelating agent and dispersant. The combined reagent package resulted in the lowest product ash value, $12.6 \%$, at a combustible recovery greater than $96 \%$. This test run also gave the highest overall separation efficiency.

Additional tests were also carried out on other individual dispersants and chelating agents. Results obtained from this work are summarized in Tables XVII and XVIII, where it will be noted that single-stage product ash rejections of the order of $30-40 \%$ are 
Table XVI. Results showing the effect that dispersant and/or chelating agent additions have on the separation performance of the $S H C$ process.

\begin{tabular}{|c|c|c|c|c|c|}
\hline Dispersant & $\begin{array}{c}\text { Chelating } \\
\text { Agent } \\
(\mathrm{lb} / \text { ton })\end{array}$ & $\begin{array}{c}\text { Product } \\
\text { Ash } \\
\text { Content } \\
(\%)\end{array}$ & $\begin{array}{c}\text { Tailing Ash } \\
\text { Content } \\
(\%)\end{array}$ & $\begin{array}{c}\text { Combustible } \\
\text { Recovery } \\
(\%)\end{array}$ & $\begin{array}{c}\text { Separation } \\
\text { Efficiency }\end{array}$ \\
\hline \hline 0.0 & 0.0 & 22.4 & 59.7 & 99.5 & $1 \%)$ \\
\hline 4.0 & 0.0 & 14.8 & 81.6 & 97.3 & 39.1 \\
\hline 0.0 & 0.4 & 13.3 & 62.4 & 90.9 & 42.9 \\
\hline 4.0 & 0.4 & 12.6 & 79.9 & 96.2 & 48.6 \\
\hline
\end{tabular}

Table XVII. Results showing the effect of dispersant additions on the separation performance of the SHC process.

\begin{tabular}{||c|c|c|c|c|c|c|}
\hline Dispersant & $\begin{array}{c}\text { Add'n } \\
\text { Rate } \\
\text { (lb/t) }\end{array}$ & $\begin{array}{c}\text { Feed } \\
\text { Ash } \\
(\%)\end{array}$ & $\begin{array}{c}\text { Prod. } \\
\text { Ash } \\
(\%)\end{array}$ & $\begin{array}{c}\text { Tail'g } \\
\text { Ash } \\
(\%)\end{array}$ & $\begin{array}{c}\text { Coal } \\
\text { Rec'y (\%) }\end{array}$ & $\begin{array}{c}\text { Sep'n } \\
\text { Eff'cy (\%) }\end{array}$ \\
\hline Sodium Silicate & 5 & 21.8 & 21.3 & 75.1 & 99.7 & 3.1 \\
\cline { 2 - 7 } & 15 & 21.4 & 16.4 & 75.1 & 97.3 & 27.2 \\
\cline { 2 - 7 } & 25 & 21.0 & 15.4 & 74.2 & 96.9 & 30.5 \\
\hline \multirow{4}{*}{\begin{tabular}{c} 
Sodium \\
\cline { 2 - 7 }
\end{tabular}} & 35 & 21.8 & 14.8 & 78.0 & 96.8 & 36.7 \\
\cline { 2 - 7 } & 4 & 20.3 & 14.8 & 81.6 & 99.2 & 31.4 \\
\cline { 2 - 7 } & 8 & 21.0 & 13.0 & 80.4 & 97.1 & 42.3 \\
\cline { 2 - 7 } & 16 & 21.2 & 13.4 & 76.2 & 96.2 & 41.0 \\
\hline & 28 & 18.5 & 11.0 & 76.0 & 96.8 & 44.3 \\
\hline
\end{tabular}

achievable, but only at excessively high reagent dosage rates ( $>5 \mathrm{lbs} / \mathrm{ton}$ ).

It should be emphasized that the data shown in Tables XVII-XVIII were obtained after only a single stage of treatment by the $S H C$ process. As a result, the ash and sulfur contents of the clean coal products could be significantly improved after several stages of 
Table XVIII. Results showing the effect of chelating agent additions on the separation performance of the SHC process.

\begin{tabular}{|c|c|c|c|c|c|c|}
\hline Chelating Agent & $\begin{array}{c}\text { Add'n } \\
\text { Rate } \\
(\mathrm{lb} / \mathrm{t})\end{array}$ & $\begin{array}{c}\text { Feed } \\
\text { Ash (\%) }\end{array}$ & $\begin{array}{c}\text { Prod. } \\
\text { Ash } \\
(\%)\end{array}$ & $\begin{array}{c}\text { Tail'g } \\
\text { Ash } \\
(\%)\end{array}$ & $\begin{array}{c}\text { Coal } \\
\text { Rec'y } \\
(\%)\end{array}$ & $\begin{array}{c}\text { Sep'n } \\
\text { Eff'y } \\
(\%)\end{array}$ \\
\hline \multirow{3}{*}{ Oxalic Acid } & 0655 & 21.6 & 21.4 & 69.3 & 99.9 & 0.7 \\
\cline { 2 - 7 } Nitrilotriacetic Acid & 6.45 & 21.7 & 13.4 & 66.6 & 93.4 & 41.0 \\
\cline { 2 - 7 } & 6.45 & 20.9 & 16.0 & 50.1 & 91.0 & 25.3 \\
\cline { 2 - 7 } & 19.42 & 20.2 & 12.0 & 25.9 & 45.2 & 21.0 \\
\hline
\end{tabular}

recleaning. This technique was used in a previous investigation ("Advanced Systems for Producing Superclean Cual," DOE Final Report, DE-AC22-86PC91221, 1990) to obtain superclean coal products in a batch SHC tests. The low rejection per stage in the SHC process can be attributed to the large amount of mineral matter entrained and/or entrapped within the settled coal coagula. The agglomerants formed by traditional oilbased agglomeration processes are considerably stronger and can withstand screening and washing steps designed to remove the entrained/entrapped particles of mineral matter. The washing step allows high grade products to be achieved in a single stage of processing. Unfortunately, as discussed in Task 3.4 , the coagula formed by the SHC process are substantially weaker and cannot be subjected to a fresh water wash without a significant degree of breakup and subsequent loss of the coal coagula. Therefore, several stages of coagulation/recleaning would be required in order to effectively redisperse the entrapped material and to dilute it to a sufficient level such that product quality is not adversely impacted.

\section{Task 3.6.2 High-Sulfur Coal Tests}

Samples of the high-sulfur Pittsburgh No. 8 seam coal were prepared for testing by grinding the raw coal for 30 minutes in a laboratory attrition mill containing $\%$-inch 
stainless steel grinding media. The ground slurry was diluted to $3 \%$ solids by weight and then introduced into the continuous sedimentation separator. Preliminary test work indicated that the Pittsburgh No. 8 seam coal coagulated very poorly above pH 7.0 due to its characteristic low hydrophobicity. Therefore, most of the tests with this coal were carried out in the neutral pH region. EDTA was added to the slurry at a concentration of $0.4 \mathrm{~g} /$ liter of suspension to complex the multivalent ions that are present in the low $\mathrm{pH}$ region and adversely affect the selectivity of the process. After the addition of the chelating agent, the $\mathrm{pH}$ was adjusted to the desired value using sodium hydroxide and conditioned for 10 minutes.

Single stage coagulation tests were conducted at $\mathrm{pH}$ values of $6.5,7.0$, and 7.8 . The feed was introduced into the center of the sedimentation tank $11 / 4$ inches from the top at a rate of $250 \mathrm{ml} / \mathrm{min}$, and the depth of the mudline was maintained at $41 / 2$ inches from the top of the cell by adjusting the rate at which the product was withdrawn from the bottom of the tank. As shown in Table XIX, the best selectivity was obtained at pH 7.0. At this $\mathrm{pH}$, the separation efficiency and product ash content were found to be only $17.2 \%$ and $10.1 \%$. A somewhat higher recovery was obtained at $\mathrm{pH} 6.5$, possibly because the coagula are larger at this $\mathrm{pH}$, but the product ash was reduced to only

Table XIX. Results illustrating the effect of $\mathrm{pH}$ on the separation of selectively coagulated Pittsburgh No. 8 coal from its associated mineral matter using an 11-inch diameter continuous sedimentation tank.

\begin{tabular}{|c|c|c|c|c|}
\hline $\mathrm{pH}$ & $\begin{array}{c}\text { Product Ash } \\
\text { Content } \\
(\%)\end{array}$ & $\begin{array}{c}\text { Tailing Ash } \\
\text { Content } \\
(\%)\end{array}$ & $\begin{array}{c}\text { Combustible } \\
\text { Recovery } \\
(\%)\end{array}$ & $\begin{array}{c}\text { Separation } \\
\text { Efficiency } \\
(\%)\end{array}$ \\
\hline 6.5 & 11.5 & 40.9 & 98.8 & 4.84 \\
\hline 7.0 & 10.1 & 27.8 & 90.5 & 17.2 \\
\hline 7.8 & 10.6 & 12.6 & 33.4 & 4.26 \\
\hline
\end{tabular}


11.5\%. At pH 7.8, the coal recovery and separation efficiency were both very poor because of the nearly complete dispersion of the particles.

The reasons for the unexpectedly poor performance of the continuous SHC process on the Pittsburgh No. 8 coal cannot be fully explained at this time. The presence of unwanted ions in solution, a characteristically lower hydrophobicity, and differences in the morphological structure of the Pittsburgh seam coal may be partly responsible for this difficulty. Additional insight into the selectivity problem may become available after completing a fundamental analysis of the coagulation of coal, quartz and clay that is planned for the companion contract No. DE-AC22-91PC91164, entitled "Development of the Selective Hydrophobic Coagulation Process."

\section{Task 3.7 Process Optimization}

In order to optimize the performance of the continuous 8-inch diameter SHC separator, a parametric study was undertaken to examine the influence of key operating parameters on process performance. In the present work, a Box-Behnken experimental test design was selected. The operating parameters examined in this work included volumetric feed flow rate, feed solids content, mudline level, and feed injection point. The test matrix required that 27 individual test runs be conducted in random order to fully ascertain the effects of these operating parameters. The operating variables, the range of values studied, and the test order for this series of tests are summarized in Table $\mathbf{X X}$.

The results of the parametric study are shown in Table XXI. As shown, product ash contents from 10.2 to $21.4 \%$ were obtained depending on the settings of the individual operating parameters. Combustible recovery values ranged from a low of 
Table XX. The Box-Behnken test design for the continuous SHC process utilizing a sedimentation tank for separating the selectively coagulated coal from its associated mineral matter.

\begin{tabular}{|c|c|c|c|c|}
\hline $\begin{array}{c}\text { Run } \\
\text { Order }\end{array}$ & $\begin{array}{l}\text { Feed Rate } \\
(\mathrm{ml} / \mathrm{min})\end{array}$ & $\begin{array}{c}\text { Solids } \\
\text { Content (\%) }\end{array}$ & $\begin{array}{l}\text { Mudline } \\
\text { (cm) }\end{array}$ & $\begin{array}{l}\text { Feed Point } \\
(\mathrm{cm})\end{array}$ \\
\hline 1 & 50.0 & 3.0 & 10.0 & 6.985 \\
\hline 2 & 50.0 & 2.0 & 11.5 & 6.985 \\
\hline 3 & 50.0 & 4.0 & 11.5 & 5.080 \\
\hline 4 & 50.0 & 2.0 & 13.0 & 5.080 \\
\hline 5 & 35.0 & 3.0 & 10.0 & 5.080 \\
\hline 6 & 50.0 & 3.0 & 11.5 & 5.080 \\
\hline 7 & 50.0 & 2.0 & 11.5 & 3.175 \\
\hline 8 & 65.0 & 3.0 & 11.5 & 3.175 \\
\hline 9 & 35.0 & 2.0 & 11.5 & 5.080 \\
\hline 10 & 35.0 & 3.0 & 11.5 & 6.985 \\
\hline 11 & 65.0 & 3.0 & 13.0 & 5.080 \\
\hline 12 & 50.0 & 4.0 & 11.5 & 3.175 \\
\hline 13 & 50.0 & 3.0 & 13.0 & 6.985 \\
\hline 14 & 50.0 & 4.0 & 13.0 & 5.080 \\
\hline 15 & 50.0 & 4.0 & 11.5 & 6.985 \\
\hline 16 & 50.0 & 3.0 & 11.5 & 5.080 \\
\hline 17 & 65.0 & 3.0 & 10.0 & 5.080 \\
\hline 18 & 50.0 & 3.0 & 13.0 & 3.175 \\
\hline 19 & 65.0 & 2.0 & 11.5 & 5.080 \\
\hline 20 & 50.0 & 2.0 & 10.0 & 5.080 \\
\hline 21 & 65.0 & 3.0 & 11.5 & 6.985 \\
\hline 22 & 35.0 & 3.0 & 13.0 & 5.080 \\
\hline 23 & 50.0 & 3.0 & 11.5 & 5.080 \\
\hline 24 & 35.0 & 3.0 & 11.5 & 3.175 \\
\hline 25 & 50.0 & 4.0 & 10.0 & 5.080 \\
\hline 26 & 65.0 & 4.0 & 11.5 & 6.985 \\
\hline 27 & 50.0 & 3.0 & 10.0 & 3.175 \\
\hline
\end{tabular}


Table XXI. Metallurgical responses for the various tests conducted in accordance with the Box-Behnken experimental design.

\begin{tabular}{|c|c|c|c|c|}
\hline $\begin{array}{c}\text { Run } \\
\text { Number }\end{array}$ & $\begin{array}{l}\text { Product Ash } \\
\text { Content (\%) }\end{array}$ & $\begin{array}{l}\text { Tailing Ash } \\
\text { Content (\%) }\end{array}$ & $\begin{array}{l}\text { Combustible } \\
\text { Recovery (\%) }\end{array}$ & $\begin{array}{c}\text { Separation } \\
\text { Efficiency (\%) }\end{array}$ \\
\hline 1 & 20.9 & 55.7 & 96.5 & 11.4 \\
\hline 2 & 12.6 & 41.9 & 76.8 & 37.0 \\
\hline 3 & 18.9 & 82.6 & 98.7 & 20.1 \\
\hline 4 & 15.9 & 51.0 & 88.9 & 29.7 \\
\hline 5 & 20.2 & 61.6 & 96.0 & 16.8 \\
\hline 6 & 17.1 & 76.5 & 97.2 & 28.1 \\
\hline 7 & 10.2 & 45.8 & 79.8 & 45.0 \\
\hline 8 & 18.4 & 73.9 & 97.8 & 19.6 \\
\hline 9 & 16.8 & 63.6 & 93.8 & 30.1 \\
\hline 10 & 17.3 & 76.0 & 96.8 & 30.0 \\
\hline 11 & 19.1 & 65.7 & 96.5 & 19.1 \\
\hline 12 & 19.5 & 82.7 & 99.1 & 14.2 \\
\hline 13 & 17.6 & 67.0 & 96.2 & 23.4 \\
\hline 14 & 19.3 & 77.5 & 98.1 & 19.8 \\
\hline 15 & 19.0 & 77.4 & 98.1 & 20.2 \\
\hline 16 & 19.8 & 74.3 & 98.4 & 14.4 \\
\hline 17 & 14.9 & 75.4 & 96.3 & 36.3 \\
\hline 18 & 11.7 & 69.5 & 92.9 & 49.6 \\
\hline 19 & 14.7 & 63.5 & 93.7 & 34.1 \\
\hline 20 & 10.5 & 49.7 & 79.3 & 48.1 \\
\hline 21 & 21.4 & 72.2 & 99.6 & 3.68 \\
\hline 22 & 18.6 & 53.6 & 93.1 & 20.4 \\
\hline 23 & 18.6 & 77.4 & 98.5 & 17.5 \\
\hline 24 & $21: 4$ & 62.7 & 98.8 & 5.77 \\
\hline 25 & 18.3 & 85.5 & 98.9 & 22.2 \\
\hline 26 & 19.8 & 83.6 & 99.3 & 11.7 \\
\hline 27 & 15.4 & 67.7 & 94.8 & 33.5 \\
\hline
\end{tabular}


$76.8 \%$ to a high of $99.6 \%$, although most values fell in the mid to upper 90 's. The following observations were derived from the test data:

- The tests resulting in low product and tailings ash contents, and low recovery values were generally associated with low feed solids content.

- High product and tailing ash contents were obtained from tests involving a small difference between the mudline depth and the feed point, from high feed rates, and from tests utilizing high feed solids content.

- High recovery values were obtained from tests involving a small difference between the mudline depth and the feed point, from high feed rates, and from tests utilizing high feed solids content.

As a step toward optimizing the operational parameter values, empirical expressions were developed to describe the effects of the process variables on product ash content, combustible recovery and separation efficiency. Three different model fits, i.e., linear, quadratic and cubic, were applied to the data to determine the most appropriate model type. Table XXII compares the predicted versus the actual for these three model types. Based on a visual comparison, the cubic model appeared to fit best, followed by the quadratic and the linear models.

A further comparison was conducted using the coefficient of multiple determination, $R^{2}$, which was calculated for each model type according to the following equation:

in which $\underline{Y}_{i}$ is the predicted response, $\bar{Y}$ is the mean of all responses, and $Y_{i}$ is the 
Table XXII. A comparison between the predicted separation efficiency values obtained from linear, quadratic and cubic models and the actual experimental values.

\begin{tabular}{|c|c|c|c|c|c|c|}
\hline \multirow[b]{2}{*}{ Run } & \multicolumn{2}{|c|}{ Linear Model } & \multicolumn{2}{|c|}{ Quadratic Model } & \multicolumn{2}{|c|}{ Cubic Model } \\
\hline & Predicted & Actual & Predicted & Actual & Predicted & Actual \\
\hline 1 & 21.6 & 11.4 & 26.6 & 11.4 & 11.4 & 11.4 \\
\hline 2 & 30.5 & 37.0 & 30.6 & 37.0 & 35.0 & 37.0 \\
\hline 3 & 15.2 & 20.1 & 16.6 & 20.1 & 20.1 & 20.1 \\
\hline 4 & 33.5 & 29.7 & 37.9 & 29.7 & 31.6 & 29.7 \\
\hline 5 & 24.2 & 16.8 & 15.4 & 16.8 & 14.9 & 16.8 \\
\hline 6 & 24.6 & 28.1 & 20.0 & 28.1 & 20.0 & 28.1 \\
\hline 7 & 37.6 & 45.0 & 45.7 & 45.0 & 43.1 & 45.0 \\
\hline 8 & 29.1 & 19.6 & 31.5 & 19.6 & 21.5 & 19.6 \\
\hline 9 & 33.2 & 30.1 & 31.1 & 30.1 & 30.1 & 30.1 \\
\hline 10 & 20.1 & 30.0 & 21.9 & 30.0 & 31.9 & 30.0 \\
\hline 11 & 25.0 & 19.2 & 17.5 & 19.2 & 17.3 & 19.2 \\
\hline 12 & 18.8 & 14.2 & 17.6 & 14.2 & 12.3 & 14.2 \\
\hline 13 & 20.5 & 23.4 & 23.6 & 23.4 & 23.4 & 23.4 \\
\hline 14 & 14.7 & 19.8 & 26.4 & 19.8 & 21.7 & 19.8 \\
\hline 15 & 11.6 & 20.2 & 19.7 & 20.2 & 18.3 & 20.2 \\
\hline 16 & 24.6 & 14.4 & 20.0 & 14.4 & 20.0 & 14.4 \\
\hline 17 & 26.0 & 36.3 & 28.8 & 36.3 & 34.4 & 36.3 \\
\hline 18 & 27.7 & 49.6 & 32.1 & 49.6 & 49.6 & 49.6 \\
\hline 19 & 34.9 & 34.1 & 31.9 & 34.1 & 34.1 & 34.1 \\
\hline 20 & 34.6 & 48.1 & 46.9 & 48.1 & 17.3 & 48.1 \\
\hline 21 & 21.9 & 3.68 & 6.47 & 3.68 & 5.58 & 3.68 \\
\hline 22 & 23.2 & 20.4 & 24.8 & 20.4 & 18.5 & 20.4 \\
\hline 23 & 24.6 & 17.5 & 20.0 & 17.5 & 35.1 & 17.5 \\
\hline 24 & 27.3 & 5.77 & 9.90 & 5.77 & 7.67 & 5.77 \\
\hline 25 & 15.7 & 22.2 & 19.4 & 22.2 & 24.1 & 22.2 \\
\hline 26 & 12.5 & 11.7 & 8.50 & 11.7 & 11.7 & 11.7 \\
\hline 27 & 28.7 & 33.5 & 31.1 & 33.5 & 20.0 & 33.5 \\
\hline
\end{tabular}




$$
R^{2}=\frac{\sum\left(\underline{Y}_{i}-\bar{Y}\right)^{2}}{\sum\left(Y_{i}-\overline{\eta^{2}}\right)^{2}},
$$

in which $\underline{Y}_{i}$ is the pridicted response, $\bar{Y}$ is the mean of all responses, and $Y_{i}$ is the observed response. If the model perfectly predicts all the actual response values, then the value of $R^{2}$ is 1 . The $R^{2}$ values for the cubic, quadratic, and linear models were found to be $0.9570,0.6903$, and 0.3433 , respectively, indicating that the cubic model provides the best fit for predicting the response of the separator. However, the pure error variance $\left(S_{8^{2}}\right)$ from the group of three repeat observations was quite high (at 51.61). Under these circumstances, the use of the model that best fits the measured responses is questionable, since models that accurately predict the measured responses of processes that lack repeatability may not represent the real process. For example, when trying to fit a model to experimental data, the resulting model line or curve should not be required to go through each data point, but it should represent the general trend of the data. For this reason, the decision was made to use the quadratic model.

Quadratic expressions were developed to predict product ash content, combustible recovery, and separation efficiency. The general form of these equations is:

$$
\begin{aligned}
\text { Response } & =C_{0}+C_{1} X_{1}+C_{2} X_{2}+C_{3} X_{3}+C_{4} X_{4}+C_{11} X_{1} X_{1}+C_{22} X_{2} X_{2} \\
& +C_{33} X_{3} X_{3}+C_{44} X_{4} X_{4}+C_{12} X_{1} X_{2}+C_{13} X_{1} X_{3}+C_{14} X_{1} X_{4}+C_{23} X_{2} X_{3} \\
& +C_{24} X_{2} X_{4}+C_{34} X_{3} X_{4}
\end{aligned}
$$

where the $C_{i j}$ 's are the coefricients and

$$
\begin{aligned}
& X_{1}=\frac{(\text { FeedRate }-50)}{15}, \\
& X_{2}=\frac{(\text { SolidsContent }-3)}{1}, \\
& X_{3}=\frac{(\text { Mudline }-11.5)}{1.5}, \\
& X_{4}=\frac{(\text { FeedPoint }-5.08)}{1.905}
\end{aligned}
$$


The list of model variables and their respective coefficients or parameter estimates are provided in Table XXIII for models representing product ash content, combustible recovery, and coagulation efficiency. Response predictions were calculated for each of the 27 tests and are compared with the measured responses in Table XXIV. The model representing combustible recovery was found to have the best fit, with a $R^{2}$ value of 0.8071 , followed by the product ash content model with a $R^{2}$ value of 0.7510 , and the separation efficiency model, with a $R^{2}$ value of 0.6903 . Although there are some large differences between the predicted and measured responses, the general trends discussed earlier in connection with the measured responses are also apparent in the predicted values. For example, low ash content predictions were obtained for tests involving a low solids content (i.e., test runs $2,4,7,9,19$, and 20), whereas product ash content values greater than $17.5 \%$ were calculated for tests involving high feed solid concentrations (i.e., test runs $3,12,14,15,25$, and 26). These trends arc also illustrated in Figures 85-88, which show the effects of the design variables (mudline and feed point) and the operating variables (feed rate and solids concentration) on the three system responses.

Detailed contour diagrams for each of the response variables are given in Appendix B. The plots for product ash content (Appendix B, Figures B1-B6), indicate that the product ash content can be minimized by operating at feed rates between 50 and $55 \mathrm{ml} / \mathrm{min}$ and at feed solids contents of approximately $2 \%$ by weight. For this range of feed rates, the feed point and mudline depth must be maintained at $3.175 \mathrm{~cm}$ and $10 \mathrm{~cm}$, respectively, in order to achieve the minimum product ash content. However, model simulations indicated that the product ash content can be improved, for feed rates less than $50 \mathrm{ml} / \mathrm{min}$, by placing the feed point $6.985 \mathrm{~cm}$ from the top of the cell and adjusting the mudline depth to $13 \mathrm{~cm}$. 


$\begin{array}{rrrrrr}\text { Response } & \text { Units } & \text { Lo } & \text { Hi } & \text { Transform } & \text { Modei } \\ \text { R1: Product Ash } & --- & -- & -- & \text { Quadratic } \\ \text { R2: } & \text { Recovery } & (\xi) & 14.000 & 20.000 & \text { Quadratic } \\ \text { R3: } & (\xi) & 80.000 & 99.000 & \text { Quadratic }\end{array}$

R1: Product Ash

R2: Recovery

R3: Seperation

Constants:

Feed Rate - $\mathbf{8 6 . 0 0}$

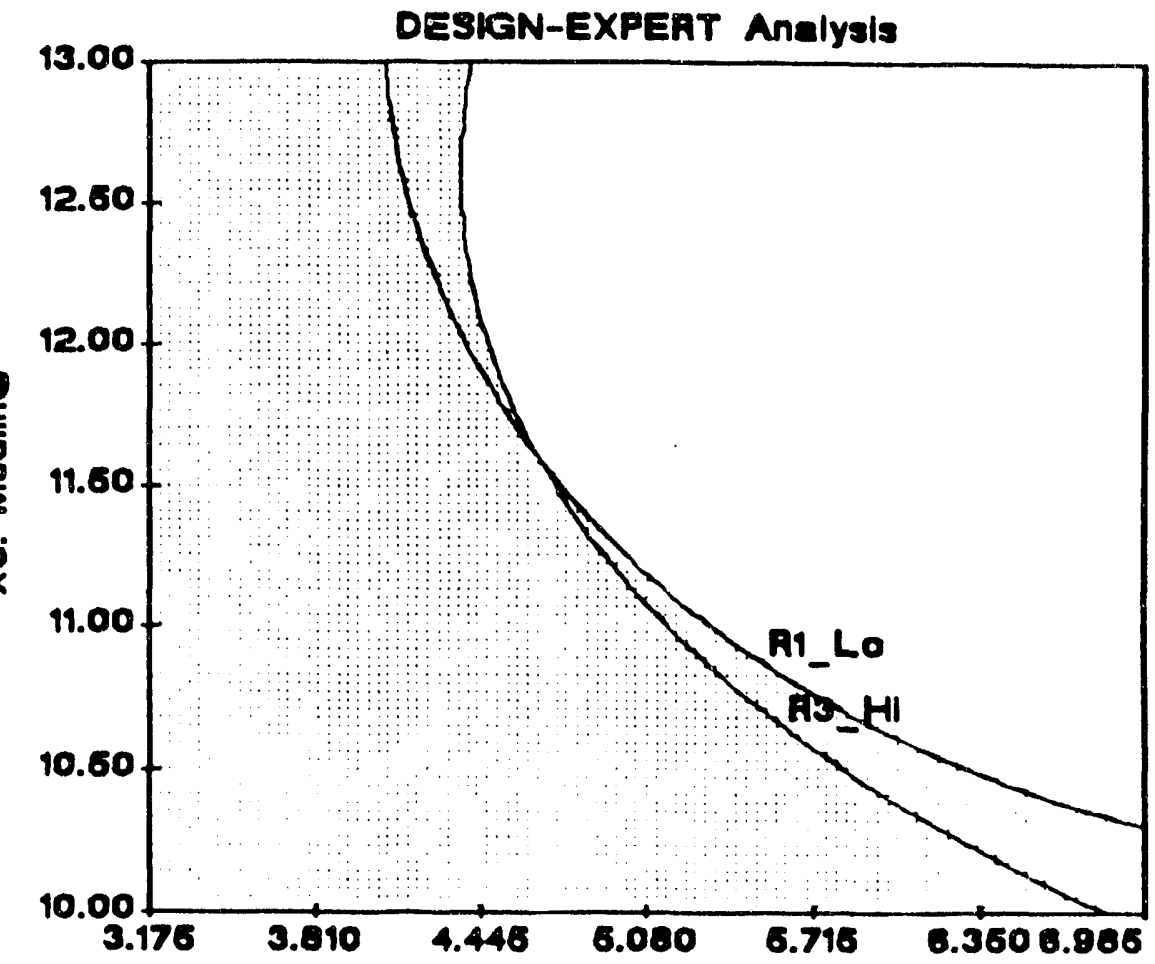

X4: Food Point

Figure 85. Process response to changes in Mudline and Feed Point at high Feed Rates. 


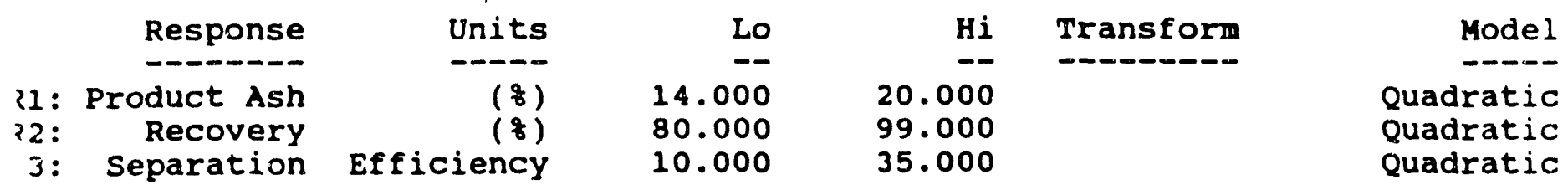

R1: Product Ash
R2: Recovery

R3: Separation

Constants:

Food Rate is 60.00

Solida Conc -2.000

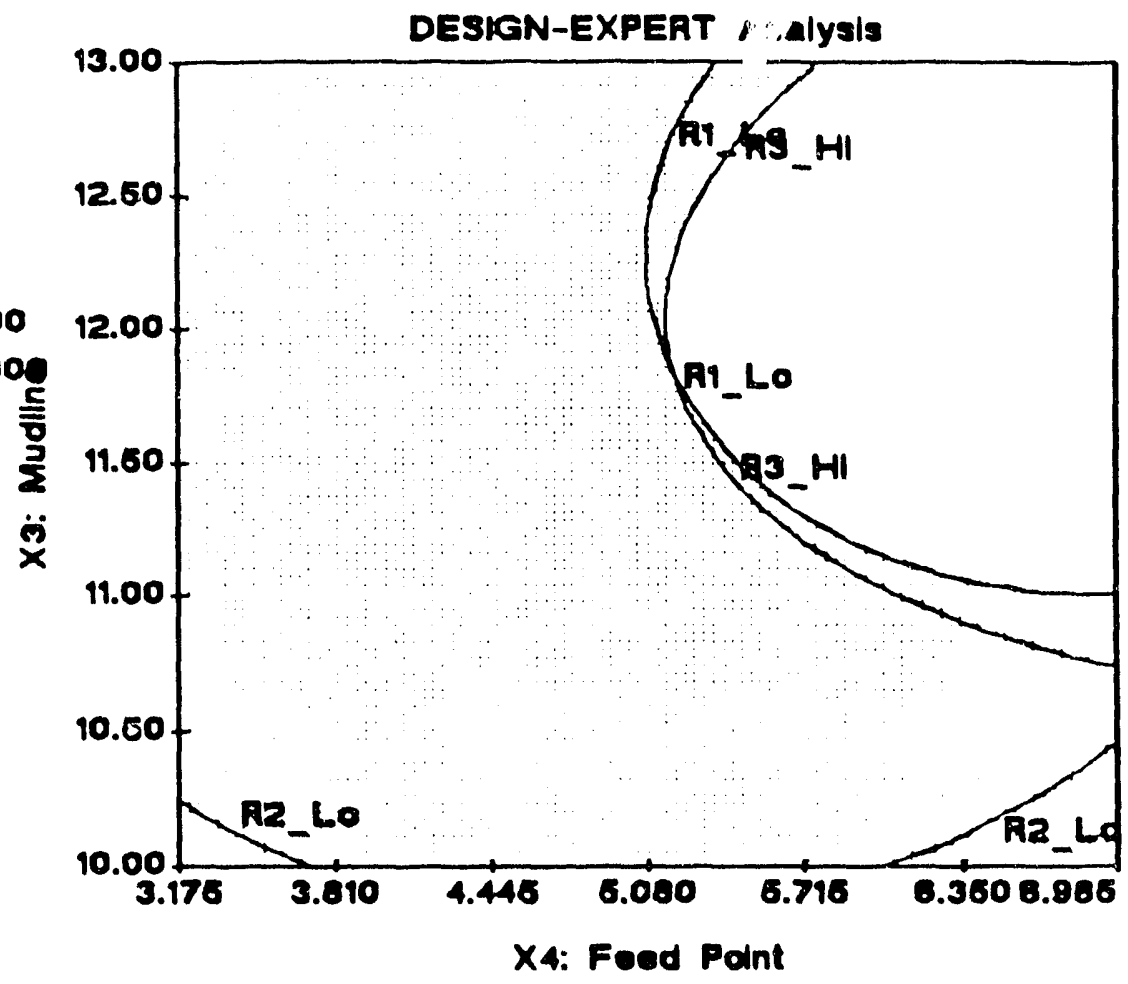

Figure 86. Process response to changes in Mudline and Feed Point at low Feed Rates. 


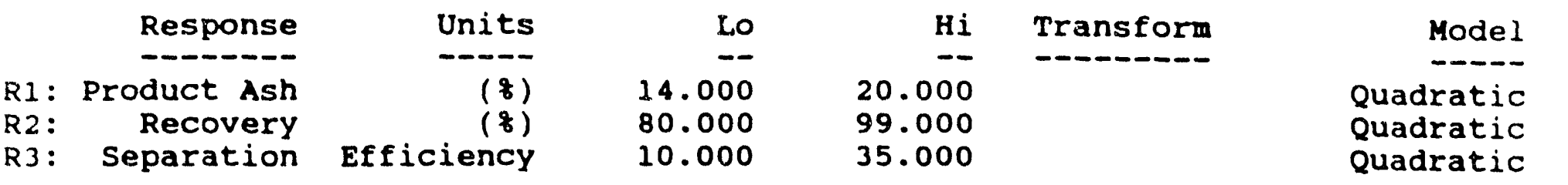

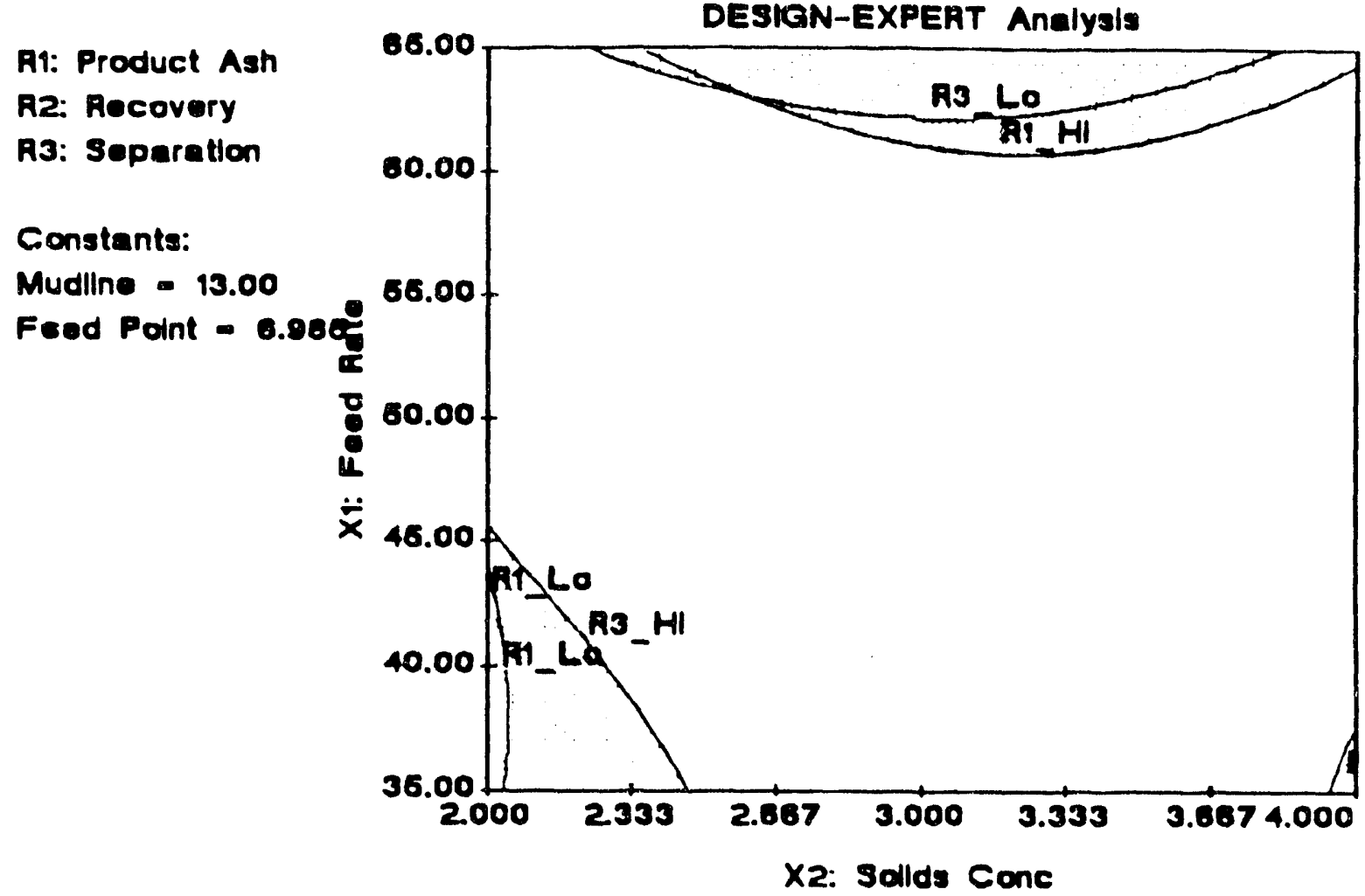

Figure 87. Process Response to changes in Feed Rate and Solids Concentration at high Mudlines and Feed Points. 


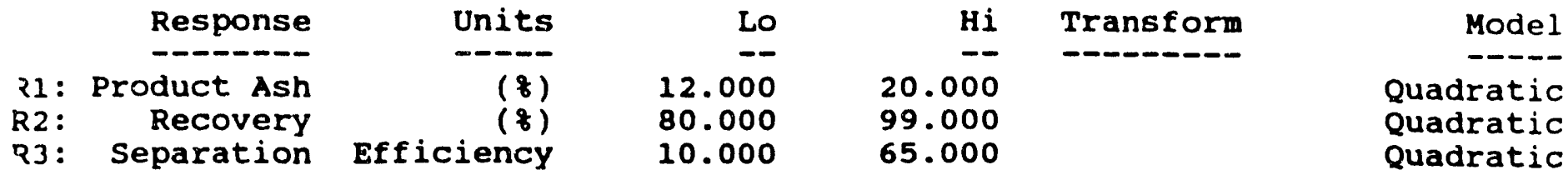

R1: Product Ash

R2: Recovery

ค3: Soparation

Constants:

Mudllne - 10.00
Feed Paint -3.176

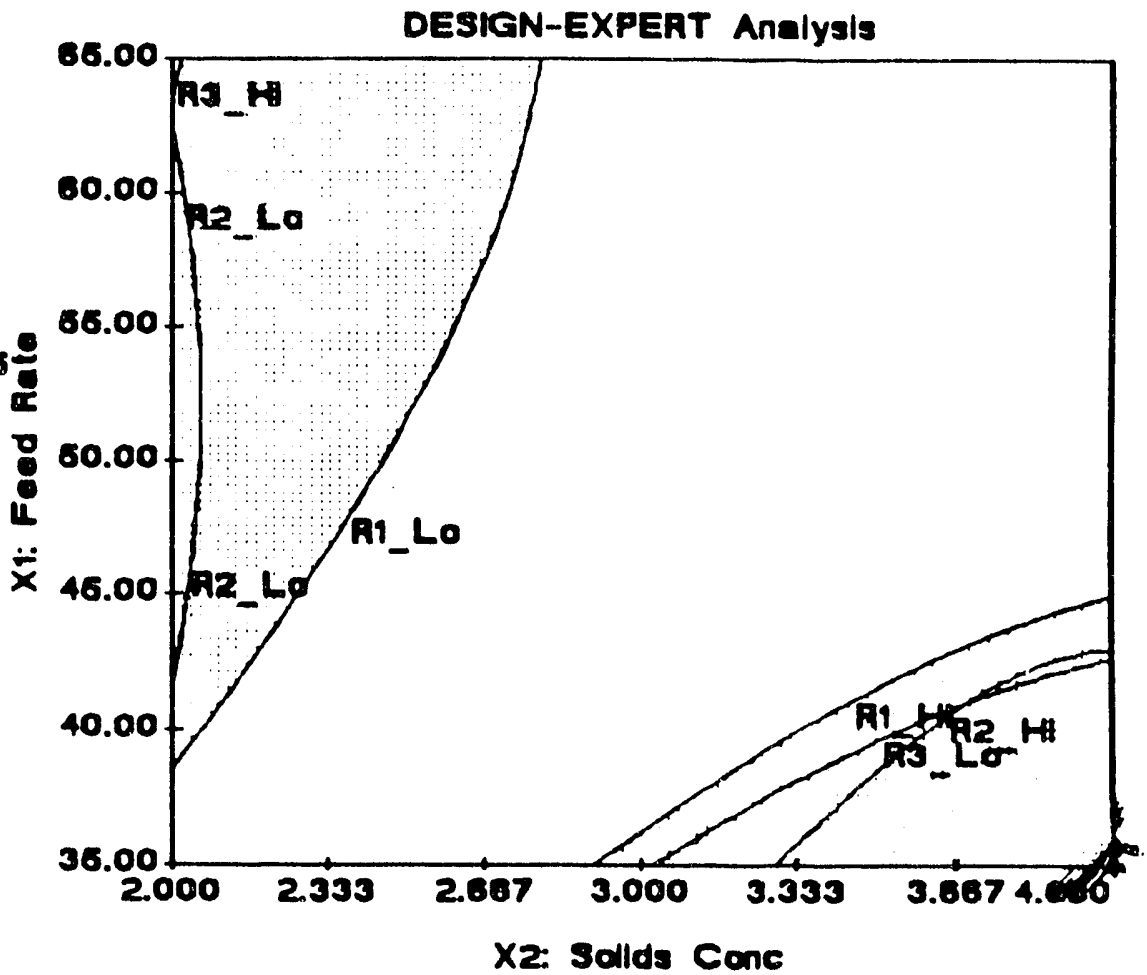

Figure 88. Process response to changes in Feed Rate and Solids Concentration at low Mudlines and Feed Points. 
Table XXIII. List of variables/coefficients for the quadratic models for product ash, recovery, and separation efficiency for the SIIC sedimentation separator.

\begin{tabular}{|c|c|c|c|}
\hline \multirow{2}{*}{ Variable } & \multicolumn{3}{|c|}{ Model Parameter Estimate } \\
\cline { 2 - 4 } & Product Ash (\%) & Recovery (\%) & Sep. Eff. (\%) \\
\hline Intercept & 18.53 & 98.00 & 20.04 \\
\hline $\mathrm{X} 1$ & -0.728 & 0.334 & 1.524 \\
\hline $\mathrm{X} 2$ & 3.007 & 7.024 & -9.751 \\
\hline $\mathrm{X} 3$ & 0.176 & 0.326 & -0.508 \\
\hline $\mathrm{X} 4$ & 0.914 & -0.053 & -3.235 \\
\hline $\mathrm{X} 1 * \mathrm{X} 1$ & 1.496 & 2.465 & -4.648 \\
\hline $\mathrm{X} 2 * \mathrm{X} 2$ & -1.855 & -4.725 & 6.333 \\
\hline $\mathrm{X} 3 * \mathrm{X} 3$ & -1.224 & -2.413 & 6.240 \\
\hline $\mathrm{X} 4 * \mathrm{X} 4$ & -0.974 & -2.636 & 2.041 \\
\hline $\mathrm{X} 1 * \mathrm{X} 2$ & -0.164 & -0.328 & 1.126 \\
\hline $\mathrm{X} 1 * \mathrm{X} 3$ & 1.448 & 0.785 & -5.178 \\
\hline $\mathrm{X} 1 * \mathrm{X} 4$ & 1.532 & 0.559 & -9.254 \\
\hline $\mathrm{X} 2 * \mathrm{X} 3$ & -1.105 & -2.580 & 3.993 \\
\hline $\mathrm{X} 2 * \mathrm{X} 4$ & -0.960 & 0.119 & 4.316 \\
\hline $\mathrm{X} 3 * \mathrm{X} 4$ & 0.105 & 0.413 & -1.015 \\
\hline
\end{tabular}

The contour plots for combustible recovery (Appendix B, Figures B7-B12), show that combustible recovery can be increased by operating at high solids contents, by locating the feed point at a depth of $5.08 \mathrm{~cm}$ and maintaining the mudline depth at 11.5 $\mathrm{cm}$. Feed rate was found to have very little effect on recovery over the range studied. However, model simulations showed a small trend which indicates that recovery is low at a feed rate of $50 \mathrm{ml} / \mathrm{min}$ and increases when the feed rate is either increased or decreased. 
Table XXIV. Results comparing the predicted response values and actual test values obtained using a continuous sedimentation separator.

\begin{tabular}{|c|c|c|c|c|c|c|}
\hline \multirow[b]{2}{*}{ Run } & \multicolumn{2}{|c|}{ Product Ash (\%) } & \multicolumn{2}{|c|}{ Combust. Recov. $(\%)$} & \multicolumn{2}{|c|}{ Separation Eff. (\%) } \\
\hline & Predicted & Actual & Predicted & Actual & Predicted & Actual \\
\hline 1 & 17.0 & 20.9 & 92.2 & 96.5 & 26.6 & 11.4 \\
\hline 2 & 14.6 & 12.6 & 83.4 & 76.8 & 30.6 & 37.0 \\
\hline 3 & 19.7 & 18.9 & 100.0 & 98.7 & 16.6 & 20.1 \\
\hline 4 & 13.7 & 15.9 & 86.8 & 88.9 & 37.9 & 29.7 \\
\hline 5 & 20.8 & 20.2 & 98.2 & 96.0 & 15.4 & 16.8 \\
\hline 6 & 18.5 & 17.1 & 98.0 & 97.2 & 20.0 & 28.1 \\
\hline 7 & 10.8 & 10.2 & 83.8 & 79.8 & 45.7 & 45.0 \\
\hline 8 & 15.9 & 18.4 & 97.7 & 97.8 & 31.5 & 19.6 \\
\hline 9 & 15.7 & 16.8 & 88.1 & 93.8 & 31.1 & 30.1 \\
\hline 10 & 19.2 & 17.3 & 96.9 & 96.8 & 21.9 & 30.0 \\
\hline 11 & 19.7 & 19.1 & 99.5 & 96.5 & 17.5 & 19.2 \\
\hline 12 & 18.8 & 19.5 & 97.6 & 99.1 & 17.6 & 14.2 \\
\hline 13 & 17.5 & 17.6 & 93.6 & 96.2 & 23.6 & 23.4 \\
\hline 14 & 17.5 & 19.3 & 95.6 & 98.1 & 26.4 & 19.8 \\
\hline 15 & 18.7 & 19.0 & 97.7 & 98.1 & 19.7 & 20.2 \\
\hline 16 & 18.5 & 19.8 & 98.0 & 98.4 & 20.0 & 14.4 \\
\hline 17 & 16.5 & 14.9 & 97.3 & 96.3 & 28.8 & 36.3 \\
\hline 18 & 15.5 & 11.7 & 92.9 & 92.9 & 32.1 & 49.6 \\
\hline 19 & 14.6 & 14.7 & 89.4 & 93.7 & 31.9 & 34.1 \\
\hline 20 & 11.2 & 10.5 & 80.9 & 79.3 & 46.9 & 48.1 \\
\hline 21 & 20.8 & 21.4 & 98.7 & 99.6 & 6.47 & 3.68 \\
\hline 22 & 18.3 & 18.6 & 97.3 & 93.1 & 24.8 & 20.4 \\
\hline 23 & 18.5 & 18.6 & 98.0 & 98.5 & 20.0 & 17.5 \\
\hline 24 & 20.4 & 21.4 & 98.1 & 98.8 & 9.90 & 5.77 \\
\hline 25 & 19.4 & 18.3 & 100.1 & 98.9 & 19.4 & 22.2 \\
\hline 26 & 20.8 & 19.8 & 100.8 & 99.3 & 8.50 & 11.7 \\
\hline 27 & 15.3 & 15.4 & 93.1 & 94.8 & 31.1 & 33.5 \\
\hline
\end{tabular}


Contour plots for separation efficiency are shown in Appendix B. These plots show that separation efficiencies can be optimized by operating at a feed rate of 60 $\mathrm{ml} / \mathrm{min}$, maintaining the solids content at $2 \%$, locating the feed point $3.175 \mathrm{~cm}$ from the top of the cell, and adjusting the mudline depth to $10 \mathrm{~cm}$. The optimum feed rate decreases when the depth of the feed point and mudline are increased. In addition, model simulations indicate that values for feed rates below $50 \mathrm{ml} / \mathrm{min}$ are larger when the depth of the feed point and mudline are decreased to $6.985 \mathrm{~cm}$ and $3.175 \mathrm{~cm}$, respectively. The contour plots also show that operating the sedimentation device at a high solids content and a low feed rate will result in low separation efficiencies.

One of the goals of this task was to determine the values for the operating parameters that would produce the lowest possible product ash content after one cleaning stage while maintaining the highest possible combustible recovery. To achieve this goal, a Hooke-Jeeves optimization technique was used to maximize separation efficiency using the quadratic model described above. The optimum values for the operating parameters were found to be the same as the values previously mentioned, namely:

$\begin{array}{ll}\text { Feed Rate } & =65 \mathrm{ml} / \mathrm{min} \\ \text { Solids Content } & =2 \% \\ \text { Mudline } & =10 \mathrm{~cm} \\ \text { Feed Point } & =3.175 \mathrm{~cm}\end{array}$

The results of four tests run to confirm this optimum are given in Table XXV. Although these results differ significantly from those calculated from the quadratic equations for the three dependent variables, i.e. product ash $=6.37 \%$, combustible recovery $=80.66 \%$, and separation efficiency $=65.62 \%$, the maximum separation 
efficiency obtained in the test series, i.e., $50.84 \%$, is greater than any value obtained in the original Box-Behnken experimental design.

Table XXV. Experimental results for tests conducted at the calculated optimum operating point for the $S H C$ sedimentation tank.

\begin{tabular}{|c|c|c|c|c|}
\hline $\begin{array}{c}\text { Feed Ash } \\
(\%)\end{array}$ & $\begin{array}{c}\text { Product Ash } \\
(\%)\end{array}$ & $\begin{array}{c}\text { Tailings Ash } \\
(\%)\end{array}$ & $\begin{array}{c}\text { Coal } \\
\text { Recovery (\%) }\end{array}$ & $\begin{array}{c}\text { Sep'n } \\
\text { Eff'cy (\%) }\end{array}$ \\
\hline 17.97 & 9.56 & 35.86 & 74.99 & 38.81 \\
\hline 20.97 & 10.11 & 46.54 & 79.84 & 45.99 \\
\hline 20.76 & 9.83 & 40.06 & 72.65 & 42.42 \\
\hline 21.16 & 9.36 & 51.32 & 82.64 & 50.84 \\
\hline
\end{tabular}




\section{SUMMARY/CONCLUSIONS}

The following conclusions can be drawn from the experimental and theoretical results presented in this investigation:

1. The selective hydrophobic coagulation $(S H C)$ process utilizes natural hydrophobicity to selectively aggregate and separate hydrophobic particles from dispersed hydrophilic particles. An extended DLVO colloid stability model which incorporates a hydrophobic interaction energy term was used to successfully explain this phenomenon. In general, $\mathrm{pH}$ modification is all that is required to induce coagulation. Separation of the coagula from the dispersed material can be accomplished by using sedimentation or sizing techniques.

2. The existence of the hydrophobic interaction energy was demonstrated at a fundamental level through the measurement of total interaction forces for two highly hydrophobic model systems, i.e., mica surfaces coated with dodecylamine and with dimethyldioctadecylammonium bromide. The coagulation behavior of fine coal cannot be explained without considering this additional attractive interaction energy.

3. Experimental resuits and theoretical calculations using existing colloidal stability models showed that maximum selectivity for the $S H C$ process occurs at a $\mathrm{pH}$ between 8 and 9 for highly hydrophobic coals. The upper limit is dictated by the dispersion of coal while the lower limit is determined by hetero- and homocoagulation of clay. Lower $\mathrm{pH}$ conditions were required for the less hydrophobic coals, which also required the addition of chelating agents to minimize the effects of cations present in these systems. 
4. The experimental study showed that a fresh Elkhom No. 3 coal suspension can be coagulated at $\zeta$-potential values as high as $-43 \mathrm{mV}$ due to the presence of an attractive hydrophobic interaction energy. The upper coagulation $\mathrm{pH}$ limit $\left(p H_{c}\right)$ and, thus, the corresponding $\zeta$-potential $\left(\zeta_{c}\right)$ was found to decrease as the surface hydrophobicity of the coal decreased. These phenomena were also explained through the use of the extended DLVO theory.

5. A population balance model was developed to describe the hydrophobic coagulation process. In this model, the rate of aggregation was estimated from the extended DLVO theory, while the rate of breakage was calculated from the particle hydrodynamics. Coagula size distributions predicted from the model were found to be in good agreement with experimentally measured size distributions.

6. Coagula size distributions were successfully measured using an in-situ particle size analyzer. The coagula $D_{s o}$ values obtained using two different coal samples were found to correlate well with the coagulation efficiency obtained over a range of $\mathrm{pH}$ values. The maximum coagula $D_{s 0}$ found for the Elkhorn No. 3 and Pittsburgh No. 8 coal suspensions was 310 and $300 \mu \mathrm{m}$, respectively.

7. A theoretical analysis showed that the kinetic energy supplied by mechanical agitation can be used to overcome the energy barrier existing between interacting particles and induce coagulation. The amount of mechanical energy required to achieve this goal was found to be a function of particle size and the magnitude of the energy barrier. However, the magnitude of the mechanical energy that can be added to the system is limited by the binding strength of the coagula. In general, if the addition of mechanical energy to the system causes the breakage rate to become greater than the coagulation rate, the coagula size distribution will decrease. 
8. Several techniques for enhancing the settling rates of the coal coagula were examined. These included the addition of small amounts of hydrocarbon oils and the use of seed particles of coarse coal or hydrophobized magnetite. The addition of coarse coal and hydrophobized magnetite nearly doubled the settling rates, while oil additions had little impact. Unfortunately, the required additions of seed particles were found to be too high for practical applications.

9. Continuous $S H C$ experiments were conducted using a sedimentation tank and a rotating drum screen for the separation of coal coagula from dispersed mineral matter. The sedimentation tank was found to be the more efficient unit, based on product ash content, combustible recovery, and separation efficiency values obtained for a given throughput per unit area. The sedimentation tank achieved ash reductions of as much as $60 \%$ in a single cleaning stage while recovering greater than $90 \%$ of the combustible material. The rotating drum screen was able to produce similar ash reductions, but recovery values were substantially lower (i.e., 60 to $70 \%$ ).

10. A parametric test program based on a Box-Behnken experimental design was conducted to optimize the process variables for an 8-inch sedimentation tank. Empirical expressions for product ash content, combustible recovery, and separation efficiency were developed to predict the effects of changes in the process operational parameters. This study found that separation efficiency was maximized when the feed rate was high $(65 \mathrm{ml} / \mathrm{min})$, the solids content was low (2\%), the feed point was at its shallowest depth $(3.175 \mathrm{~cm})$, and the mudline level was high $(10 \mathrm{~cm})$.

11. The optimum feed flow rate for the 11-inch diameter sedimentation tank was found to be between 250 and $350 \mathrm{ml} / \mathrm{min}$ (i.e., $1.5-2.1 \mathrm{lbs} / \mathrm{hr} / \mathrm{ft}^{2}$ at $3 \%$ solids). 
Larger foed rates resulted in a decrease in recovery whereas smaller feed rates increased the ash content in the product. These values were found to correlate well with the residence time of $\mathbf{3 0}$ to $\mathbf{4 0}$ minutes required for the settling of the coal coagula into the product process stream.

12. For the 11 -inch diameter sedimentation tank, the solids content corresponding to maximum separation efficiency was found to be $3 \%$. A high solids content resulted in an increase in the product ash content whereas a lower solids content resulted in a decrease in recovery.

13. Particle size was also found to be an important process parameter. Recovery and product ash content values improved with decreasing particle size, presumably because of improved liberation and increased dominance of the surface forces at the finer grind sizes. 


\section{REFERENCES}

Attard, P., 1989, "Long-range attraction between hydrophobic surfaces," J. Phys. Chem., 93, pp. 6441-6444.

Barcicki, J., Waksmundzki, A., and Maraszak, E., 1962, "A new method for the direct measurement of adhesion force between mineral particle and air bubble in an elementary flotation process," Chemia Stosowana, 6, p. 99.

Bargeman, D. and Vader, F.V., 1972, "van der Waals forces between immersed particles," J. Electroanal. Chem., 37, p. 45.

Blake, T.D., 1972, "Equilibrium wetting films of n-alkanes on $\alpha$-alumina", JCS Faraday Trans. I, 71, p. 208.

Blake, T.D. and Kitchener, J.A., 1972, "Stability of aqueous films on hydrophobic methylated silica", JCS Faraday Transactions I, 68, p. 1435.

Blake, P. and Ralston, J., 1985, "Controlled methylation of quartz particles", Colloids Surfaces, 15, p. 108.

Broadbent, S.R. and Callcott, T.G., 1956, "Coal breakage processes," J. Inst. Fuel, 29, p. 524.

Broadbent, S.R. and Callcott, T.G., 1957, "Coal breakage processes," J. Inst. Fuel, 30, p. 13.

Capes, C.E., 1989, "Liquid phase agglomeration: Process opportunities for economic and environmental challenges," in: Challenges in Mineral Processing (K.V.S. Sastry and D.W. Fuerstenau, eds.), Society of Mining Engineers, Littleton, Colorado, pp. 237-251.

Cheh, C.H., Glass, R.W. and Sehgal, R., 1982, "Solvent recovery for the oil agglomeration coal cleaning process," SME-AIME Annual Meeting, Dallas, Texas, February 14-18; Preprint No. 82-48.

Chia, Y.H. and Somasundaran, P., 1983, "A theoretical approach to flocculation in carrier flotation for beneficiation of clay," Colloids and Surfaces, 8, pp. 187-202.

Christenson, H.K., 1988, J. Dispersion Sci. Technol., 9, p. 171.

Christenson, H.K., Claesson, P.M., Berg, J., and Herder, P.C., 1989, "Forces between fluorocarbon surfactant monolayers: Salt effects on the hydrophobic interaction", J. Phys. Chem., 93, p. 1472.

Christenson, H.K. Claesson, P.M., and Pashley, R.M., 1987, Proc. Indian Acad. Sci. Chem. Sci., 98, p. 379. 
Claesson, P.M., 1987, Prog. Colloid Polym. Sci., 74, p. 48.

Claesson, P.M., Blom, C.E., Herder, P.C. and Ninham, B.W., 1986, J. Colloid Interface Sci., 114, p. 235.

Claesson, P.M. and Christenson, H.K., 1988, J. Phys. Chem., 92, p. 1650.

Dai, Q., Sasaki, H., Usui, S., and Kaisheva, M., 1990, "Effect of sodium dodecyl sulfate concentration on the attachment between mercury and argon bubbles in aqueous solutions", J. Colloid Interface Sci., 139, p. 30.

Delichatsios, M.A. and Probstein, R.F., 1975, J. Colloid Interface Sci., 51, p. 394.

Derjaguin, B.V., 1934, Kolloid Z., 69, p. 155.

Derjaguin, B.V., 1989, Theory of Stability of Colloids and Thin Films (English Translation), Consultants Bureau, New York.

Derjaguin, B.V. and Landau, L., 1941, Acta Phys. Chim. USSR, 14, p. 633.

Derjaguin, B.V., Rabinovich, Ya.I. and Churaev, N.V., 1978, Nature (London), 272, p. 313.

Devanathan, M.A.V. and Tilak, B.V.L.S.R.A., 1965, Chem. Review, 65, p. 635.

Drzymala, J., Markuszewski, R. and Wheelock, T.D., 1986, "Influence of air on oil agglomeration of carbonaceous solids in aqueous suspension," Int. J. Miner. Process., 18, pp. 277-286.

Eriksson, J.C., Ljunggren, S., and Claesson, P.M., 1989. "A phenomenological theory of long-range hydrophobic attraction forces based on a square-gradient variational approach," JCS Faraday Trans. II, 85, p. 163.

Firth, B.A. and Hunter, R.J., 1976, J. Colloid Inserface Sci., 57, pp. 248-256.

Fowkes, F.M., 1964, "Attractive forces at interfaces," Indian Engineering and Chemistry, 56, p. 40.

Frens, G. and Overbeek, J.T.G., 1972, J. Colloid Interface Sci., 38, pp. 376-387.

Hamaker, H.C., 1937, "The London-van der Waals attraction between spherical particles," Physica, 4, p. 328.

Herder, P.C., 1990, J. Colloid and Interface Sci., 134, p. 346.

Hough, D.B. and White, L.R., 1980, "The calculation of Hamaker constants from Lifshitz theory with applications to wetting phenomena", Adv. Colloid Interface Sci., 14, p. 3. 
Israelachvili, J.N. 1978, Faraday Discuss. Chem. Soc., 65, p. 20.

Israelachvili, J.N., 1985, Intermolecular and Surface Forces: With Applications to Colloidal and Biological Systems, Academic Press Inc., London, 1985.

Israelachvili, J.N. and McGuiggan, P.M., 1988, "Forces between surfaces in liquids," Science, 241, pp. 795-800.

Israelachvili, J.N. and Pashley, R., 1982, Nature (London), 300, p. 341.

Israelachvili, J.N. and Pashley, R.M., 1984, J. Colloid Interface Sci., 98, p. 500.

Jańczuk, B., 1983, "The effect of n-alkanes on the force of air bubble detachment from a quartz surface in water", Powder Technology, 34, p. 243.

Janczuk, B., 1989, "The effect of n-alkanes on the force of air bubble detachment from a quartz surface in water," Powder Technology, 34, p. 243.

Keller, D.V., Jr., 1984, "Methods for processing coal," U.S. Patent Number $4,484,928$.

Keller, D.V., Jr. and Burry, W.M., 1989, "The demineralization of coal using selective agglomeration by the T-process," Coal Preparation, 6.

Kitchener, J.A., 1984, "Surface forces in flotation - a critique", in Principles of Mineral Flotation, ed. by M.H. Jones and J.T. Woodcock, The Australian Institute of Mining and Metallurgy, Victoria, Australia, p. 65.

Koh, P.T.L., Uhlherr, P.H.T. and Andrews, J.R.G., 1985, "The effect of capillary condensation and liquid bridging on the bonding of hydrophobic particles in shearflocculation," J. Colloid Interface Sci., 108 (1), pp. 95-103.

La Mer, V.K. and Healy, T.W., 1963, "The role of filtration in inv:stigating flocculation and redispersion of colloidal dispersions," J. Phys. Chem., 67 (11), pp. 2417 2420.

La Mer, V.K. and Healy, T.W., 1966, "The nature of the flocculation reactions in solid-liquid separation," Solid-Liquid Separation (Pool, J.B. and Doyle, D., eds.) London, Her Majesty's Stationary Office, pp. 44-59.

J.S. Laskowski, 1986, "The relationship between flotability and hydrophobicity", in Advances in Mineral Processing, ed. by P. Somasundaran, AIME Inc., New York, p. 189.

Laskowski, J.S. and Iskra, J., 1970, "Role of capillary effects in bubble-particle collision in flotation," Trans. $I M M$, Sec. C, 79, p. 6.

Laskowski, J.S. and Kitchener, J.A., 1969, "The hydrophilic-hydrophobic transition on silica", J. Colloid Interface Sci., 29, p. 670. 
Laskowski, J.S., Xu, Z., and Yoon, R.-H., 1991, "Energy barrier in particle-to-bubble attachment and its effect on flotation," 17th Intermational Mineral Processing Congress, Dresden.

Maynard, R.N. and Skipper, B.R., 1968, "Method of beneficiating clay by removal of titanium impurities," U.S. Patent Number 3,371,988.

Nagata, S., 1983, in Mixing: Principles and Applications, Halstead Press, New York.

Pashley, R.H., 1981, J. Colloid Interface Sci., 80, p. 153.

Pugh, R.J. and Kitchener, J.A., 1972, "Experimental confirmation of selective coagulation in mixed colloidal suspensions," J. Colloid Interface Sci., 35(4), p. 656-664.

Rabinovich, Ya.I., and Derjaguin, B.V., 1987, Kolloidn. Zh., 49, p. 682.

Rabinovich, Ya.I., and Derjaguin, B.V., 1988, Colloids Surf., 30, p. 243.

Read, A.D. and Kitchener, J.A., 1969, "Wetting films on silica", J. Colloid Interface Sci., 30, p. 391.

Read, R.B., Rapp, D.M., Camp, L.R., Summers, M.S., Ruch, R.R., Demaris, P.J., Ehrlinger, H.P., Meyers, C.Y., and Fitzpatrick, J.A., 1987, "Advanced physical fine coal cleaning: Developments in pyritic sulfur and ash reduction of Illinois coals by ISGS aggregate flotation," in Processing and Utilization of High Sulfur Coals II, (Y.P. Chugh and R.D. Caudle, Eds.), Elsevier Science Publishing, p. 83.

Ruckenstein, E. and Churaev, N., 1991, "A possible hydrodynamic origin of the forces of hydrophobic attraction", J. Colloid Interface Sci., 147, p. 535.

Schulze, H.J., 1984, Developments in Mineral Processing, Elsevier, Amsterdam.

Usui, S., Sasaki, H., and Hasegawa, F., 1986, "Attachment between mercury and argon bubbles in aqueous KF solutions", Colloids Surfaces, 18, p. 53.

van Olphen, H., 1977, An Introduction to Clay Colloid Chemistry, 2nd edition, John Wiley and Sons, New York, Chapter 11.

Verwey, E.J.W. and Overbeek, J.Th.G., 1948, Theory of the Stability of Lyophobic Colloids, Elsevier, Amsterdam.

Vincent, B., 1973, J. Colloid Interface Sci., 42, p. 270.

Warren, L.J., 1975, J. Colloid Interface Sci., 50, pp. 307-318.

Warren, L.J., 1977, "The stability of suspensions. A guide for beginners," Chem. Aust., 44, pp. 315-318. 
Warren, L.J., 1981, "Shear flocculation," Chemtech, March, pp. 180-185.

Warren, L.J., 1982, "Flocculation of stirred suspensions of cassiterite and tourmaline," Colloids and Surfaces, 5, pp. 301-319.

Warren, L.J., 1984, "Ultrafine particles in flotation," Principles of Mineral Flotation (Proceedings of the Wark Symposium), Austr. Inst. Mining, Brisbane, pp. 185213.

Wojcik, W. and Al Taweel, A.M., 1984, "Beneficiation of Coal Fines by Aggregative Flotation," Powder Technology, 40, pp. 179-185.

Xu, Z., 1990, "A Study of Hydrophobic Interaction in Fine Particle Coagulation," Ph.D. Dissertation, Department of Mining and Minerals Engineering, Virginia Polytechnic Institute and State University, Blacksburg, Virginia.

$\mathrm{Xu}, \mathrm{Z}$. and Yoon, R.-H., 1989, "The role of hydrophobic interactions in coagulation," J. Colloid Interface Sci., 132(2), pp. 532-541.

Xu, Z. and Yoon, R.-H., 1990, "A study of hydrophobic coagulation," J. Colloid Interface Sci.; in press.

Yoon, R.-H., 1991, "Hydrodynamic and surface forces in bubble-particle interactions," Proceedings of the XVII International Mineral Processing Congress, Dresden, pp. 17-31.

Yoon, R.-H. and Yordan, J.L., 1986, "Zeta potential measurements on microbubbles generated using various surfactants," J. Coll. Inter. Sci., 113, p. 430.

Yordan, J., 1989, "Studies on the stability of thin films on bubble-particle adhesion," Ph.D. Thesis, Department of Mining and Minerals Engineering, Virginia Polytechnic Institute and State University, Blacksburg, Virginia.

Yordan, J.L. and Yoon, R.-H., 1988, "Induction time measurements for a coal flotation system", in Interfacial Phenomena in Biotechnology and Materials Processing (Process Technology Proceedings, 7), ed. by Y.A. Allia, B.M. Moudgil and S. Chander, Elsevier Science Publishers, New York, p. 333.

Yordan, J.L. and R.-H. Yoon, 1991, "The critical rapture thickness of thin water films on hydrophobic surfaces", J. Colloid Interface Sci., 146, p. 565.

Young, T., 1855, Miscellaneous Works, (G. Peacock, Ed.), 1, Murray, London, p. 418. 
APPENDIX A: SEM-IPS and Centrifugal Washability Data

A-1 


\section{ELKHORN FEED}

$\begin{array}{lcccc}\text { GRADE } & \text { G COAL } & \text { G PYR } & \text { G MIN } & \text { Wt\% } \\ >95 & 98.5955 & .051584 & 1.25295 & 39.3206 \\ 95 \times 80 & 89.5106 & 1.27471 & 9.21467 & 22.5240 \\ 80 \times 60 & 71.6680 & 2.97732 & 25.3547 & 8.98919 \\ 60 \times 40 & 49.5238 & 2.25773 & 48.2185 & 7.63046 \\ 40 \times 20 & 31.1586 & 3.65393 & 65.1875 & 3.19422 \\ 20 \times 5 & 12.0235 & 1.42535 & 86.5511 & 8.33322 \\ <5 & 1.88358 & 4.04493 & 94.0715 & 5.90806 \\ \text { WT \% } & 72.0119 & 1.39301 & 26.5951 & \end{array}$

\begin{tabular}{|c|c|c|c|c|}
\hline & & $65 \times 100$ & IESH & \\
\hline $\begin{array}{l}\text { GRADE } \\
>95\end{array}$ & $\begin{array}{l}\text { G COAL } \\
98.617 \mathrm{~s}\end{array}$ & $\begin{array}{l}\text { G PYR } \\
.089023\end{array}$ & $\begin{array}{l}\text { G MIN } \\
31.29371\end{array}$ & $\begin{array}{c}\text { Wt\% } \\
41.8296\end{array}$ \\
\hline $95 \times 80$ & 89.2469 & 1.87434 & 8.87878 & 18.4347 \\
\hline $80 \times 60$ & 70.6047 & 4.29029 & 25.1050 & 9.70761 \\
\hline $60 \times 4$ & 49.7355 & 4.58496 & $6 \quad 45.6795$ & 6.62402 \\
\hline $40 \times 20$ & 28.7928 & 3.68565 & $5 \quad 67.5215$ & 7.81016 \\
\hline $20 \times 5$ & 11.1040 & 5.35728 & 883.5387 & 477 \\
\hline$<5$ & 2.22744 & 6.9 & 290.8107 & 10 \\
\hline T $\%$ & 71.2476 & 2.33195 & 526.4205 & \\
\hline & & $100 \times 150$ & MESH & \\
\hline 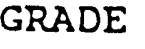 & COAL & G PYR & G MIN & Wt: \\
\hline 95 & 98.6567 & .050742 & 21.29260 & 41.7009 \\
\hline $95 \mathrm{X}$ & 89.3527 & 1.89293 & 38.75441 & 19.0516 \\
\hline $80 x$ & 71.4465 & 54.57265 & $5 \quad 23.9809$ & 9.32015 \\
\hline $60 \mathrm{X}$ & 49.9222 & 4.47874 & $4 \quad 45.5992$ & 7.201013 \\
\hline $40 \times 20$ & 30.5967 & 8.31082 & 01.0925 & 7.00248 \\
\hline $20 x$ & 12.2347 & 77.84556 & $560.91 \subseteq 7$ & 3.25207 \\
\hline$<5$ & 1.75472 & 229.4372 & 278.3081 & j. 5547. \\
\hline & 1. & & & \\
\hline
\end{tabular}




\section{ELKRORN FEED}

\begin{tabular}{lcccc}
\multicolumn{5}{c}{ 150X200 MESH } \\
GRADE & G COAL & G PYR & G MIN & Wt\% \\
$>95$ & 98.72237 & .0218833 & 1.255747 & 42.97760 \\
$95 \times 80$ & 89.49729 & 1.705643 & 8.797062 & 15.82483 \\
$80 \times 60$ & 71.13518 & 5.132405 & 23.73241 & 6.740199 \\
$60 \times 40$ & 49.70647 & 6.096394 & 44.19714 & 6.722422 \\
$40 \times 20$ & 29.91180 & 5.156219 & 64.93198 & 7.073068 \\
$20 \times 5$ & 11.13169 & 9.625351 & 79.24296 & 10.85155 \\
$<5$ & 1.860984 & 14.34460 & 83.79441 & 9.796280 \\
& & & &
\end{tabular}

\begin{tabular}{|c|c|c|c|c|}
\hline & & $200 \times 270 \mathrm{M}$ & IESH & \\
\hline $\begin{array}{l}\text { GRADE } \\
>95\end{array}$ & $\begin{array}{l}\text { G COAL } \\
99.05290\end{array}$ & $\begin{array}{l}\text { G PYR } \\
.0178004\end{array}$ & $\begin{array}{l}\text { G MIN } \\
.9292949\end{array}$ & $\begin{array}{l}\text { Wt\% } \\
46.22485\end{array}$ \\
\hline & 89.12686 & $; 1.670462$ & 9.202679 & 13.33791 \\
\hline 0 & 71.16252 & 4.255668 & 324.58181 & 7.508143 \\
\hline 640 & 49.57453 & 37.182022 & 433.24344 & 6.863806 \\
\hline 220 & 27.86383 & 4.841717 & 67.29445 & 8. \\
\hline$\times 5$ & 11.32665 & 9.485743 & 79.18761 & 196 \\
\hline & 1.918916 & 522.63513 & 75.44 & 8. \\
\hline$\%$ & 69.88807 & 4.284859 & 25.82707 & \\
\hline & & $270 \times 400 \mathrm{M}$ & IESH & \\
\hline 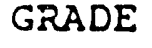 & G COAL & G PYR & G MIN & Wt: \\
\hline & 99.08481 & .0178735 & .8973144 & 46.13174 \\
\hline $\mathrm{x}$ & 89.48365 & ;.9833149 & 9.533039 & 9.554721 \\
\hline $1 \times 60$ & 70.37931 & 4.190314 & 25.43047 & 6.251310 \\
\hline 0 & 50.29790 & 4.945316 & 44.75678 & 4.397684 \\
\hline$x$ & 28.70025 & 511.64692 & 59.55283 & 6.605958 \\
\hline & 11.06808 & 317.30482 & 71.62711 & $9.33=538$ \\
\hline & 1.284552 & 48.20508 & 50.51036 & 17.32705 \\
\hline & 64.00732 & 612 & 201 & \\
\hline
\end{tabular}




\section{ELKHORN FEED - 400 MESH}

$\begin{array}{lrrrrr}\text { GRADE } & \text { G COAL } & \text { G } & \text { PYR } & \text { G MIN } & \text { WT } \% \\ >95 & 97.81974 & & 0 & 2.180257 & 31.7729 ? \\ 95 \times 80 & 89.84866 & .2115590 & 9.939785 & 22.85056 \\ 80 \times 60 & 70.10194 & .0759058 & 29.82216 & 7.392371 \\ 60 \times 40 & 53.74871 & .4400636 & 45.81123 & 3.275053 \\ 40 \times 20 & 30.95685 & 13.63557 & 55.40758 & 16.27064 \\ 20 \times 5 & 9.398297 & 18.76882 & 71.83288 & 17.53797 \\ <5 & 0 & 0 & 0 & 0\end{array}$

WT \& $\quad 66.10690 \quad 5.567110 \quad 28.32599$

\begin{tabular}{|c|c|c|c|c|}
\hline \multirow[b]{2}{*}{ GRADE } & \multicolumn{2}{|r|}{$26 \times 19$ uM } & \multirow[b]{2}{*}{ G MIN } & \multirow[b]{2}{*}{ WT $\%$} \\
\hline & G COAL & G PYR & & \\
\hline 95 & 97.97714 & 0 & 2.022857 & 26.6258 \\
\hline $5 \times 80$ & 89.05779 & .0394201 & 90279 & 22.9639 \\
\hline 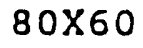 & 72.30604 & .4672 & 2593 & 7.20767 \\
\hline$\partial X$ & 50.98507 & 0 & 49.01493 & 9.24162 \\
\hline 020 & 30.90017 & 1.418535 & 67.68130 & 14.2874 \\
\hline $0 x$ & 12.5 & 2.56 & 84.44423 & 16.6480 \\
\hline & 2.955060 & .9575 & 96.08739 & 3.02533 \\
\hline se & 63.12 & & 36.16970 & \\
\hline
\end{tabular}

\begin{tabular}{|c|c|c|c|c|}
\hline & & $19 \times 13 \mathrm{uM}$ & & \\
\hline GRA & G COAL & G PYR & G MIN & WT $\%$ \\
\hline 5 & 98.51270 & .0184567 & 1.468842 & 26.65272 \\
\hline $2 \times 8$ & 89.05955 & .0675581 & 10.37289 & 16.29736 \\
\hline$\times 60$ & 70.17039 & .5713208 & 29.25829 & 20.64306 \\
\hline$\times 40$ & 49.54072 & 0 & 5928 & 5.821931 \\
\hline 0 & 27.76925 & .7269370 & 0381 & 26.32898 \\
\hline$\times 5$ & 13.07312 & 2.444964 & $84.481 \subseteq 1$ & 27.25692 \\
\hline & 2.766144 & 39.76836 & 57.46550 & 6.40904 \\
\hline & 58.24005 & 8 & & \\
\hline
\end{tabular}




\section{EIKHORN FEED -400 MESH}

\begin{tabular}{|c|c|c|c|c|}
\hline & COAL & $\begin{array}{l}13 \times 9.3 \text { UM } \\
\text { G PYR }\end{array}$ & E MIN & WT $\frac{f}{6}$ \\
\hline & 98.71905 & 5 & 1.280952 & 25.896 \\
\hline & 88.79439 & .0885951 & 11.11701 & 17. \\
\hline X60 & 69.72207 & 1.377968 & 28.89996 & 12.610 \\
\hline $1 \times 40$ & 47.63323 & 3.829719 & 3705 & 13 \\
\hline $\mathrm{x}$ & 30.94607 & 3.1 & 65. & 13 \\
\hline$\partial x$ & 13.42414 & 7.311168 & 79.26470 & 10.70285 \\
\hline & 2.437164 & 10.77057 & 86.79226 & 6.04781 \\
\hline & 62.23990 & 2.576826 & 18328 & \\
\hline
\end{tabular}

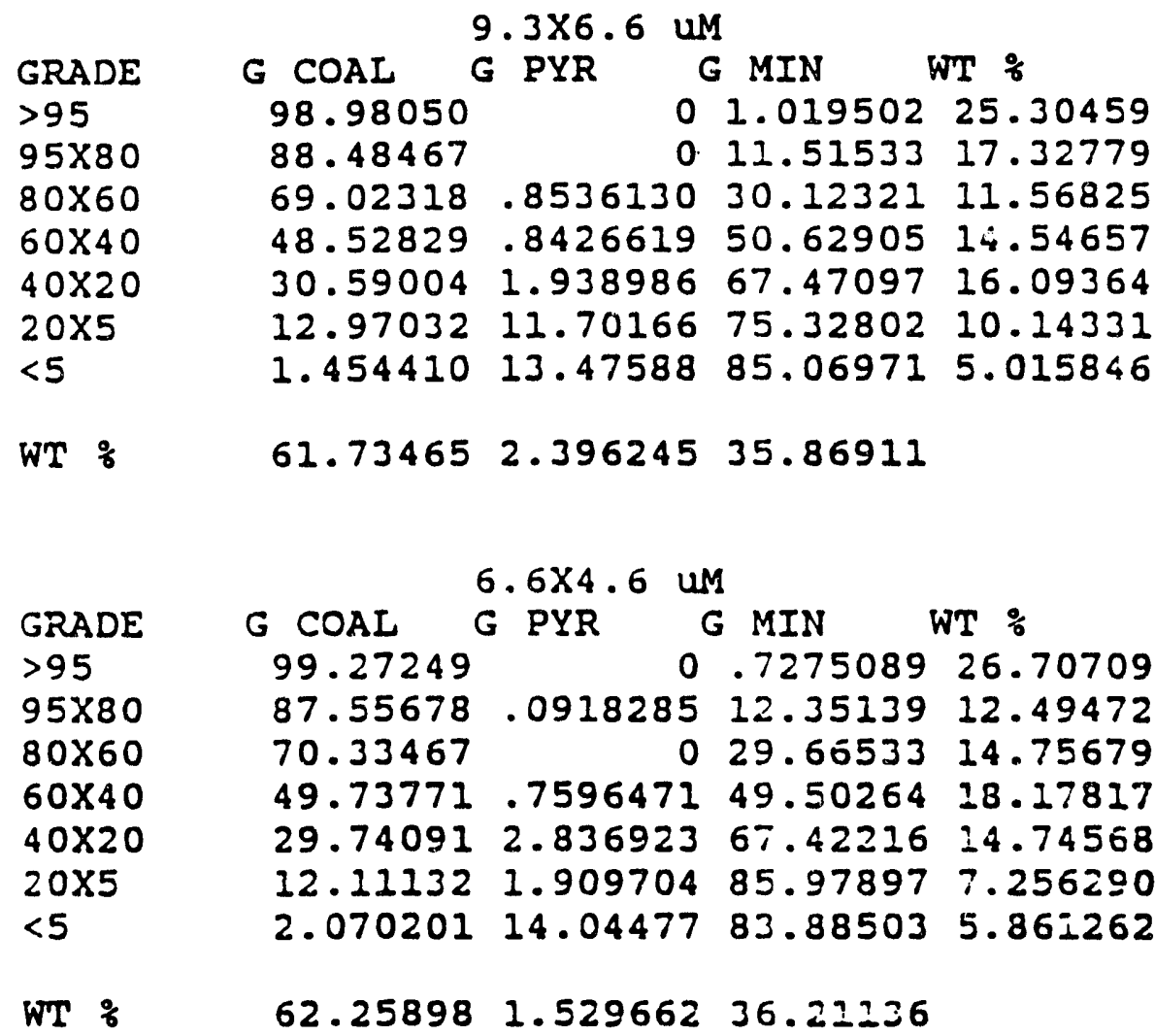


ELKHORN FEED -400 MESH

\begin{tabular}{lccrl} 
& \multicolumn{4}{c}{$4.6 \times 3.3$} \\
GRADE & G COAL & G PYR & G MIN & WT $\%$ \\
$>95$ & 99.56077 & 0 & .4392335 & 30.87321 \\
$95 \times 80$ & 87.24168 & 0 & 12.75832 & 11.20129 \\
$80 \times 60$ & 69.76319 & 0 & 30.23681 & 15.42267 \\
$60 \times 40$ & 49.86896 & 0 & 50.13104 & 14.40764 \\
$40 \times 20$ & 31.11503 & 1.827286 & 67.05769 & 11.71640 \\
$20 \times 5$ & 11.63522 & 5.648552 & 82.71622 & 7.798097 \\
$<5$ & 1.374108 & 26.97833 & 71.64757 & 8.470685 \\
WT $\%$ & 63.21759 & 2.939821 & 33.84259
\end{tabular}

\begin{tabular}{|c|c|c|c|c|}
\hline & G COAL & $\begin{array}{l}3.3 \times 2.3 \text { u } \\
\text { G PYR }\end{array}$ & G MIN & WT $\%$ \\
\hline & $\begin{array}{l}99.81803 \\
87.84661\end{array}$ & $\begin{array}{l}0 \\
0\end{array}$ & $\begin{array}{r}1819659 \\
12.15339\end{array}$ & $\begin{array}{r}41.94920 \\
8.378919\end{array}$ \\
\hline $80 \times 60$ & 69.42440 & .2277401 & 30.34786 & 11.31376 \\
\hline $0 \times 40$ & 49.69874 & .3233169 & 49.97794 & 12.40165 \\
\hline $0 \times 20$ & 30.35421 & 0 & $69.6457 c$ & 9.07921 .5 \\
\hline $0 \times 5$ & 12.09412 & 25.703014 & 82.20286 & 58.368826 \\
\hline & 1.565023 & 314.35550 & $84.0794 \varepsilon$ & 8.508432 \\
\hline & 67.15266 & 51.764566 & 31.08278 & \\
\hline
\end{tabular}

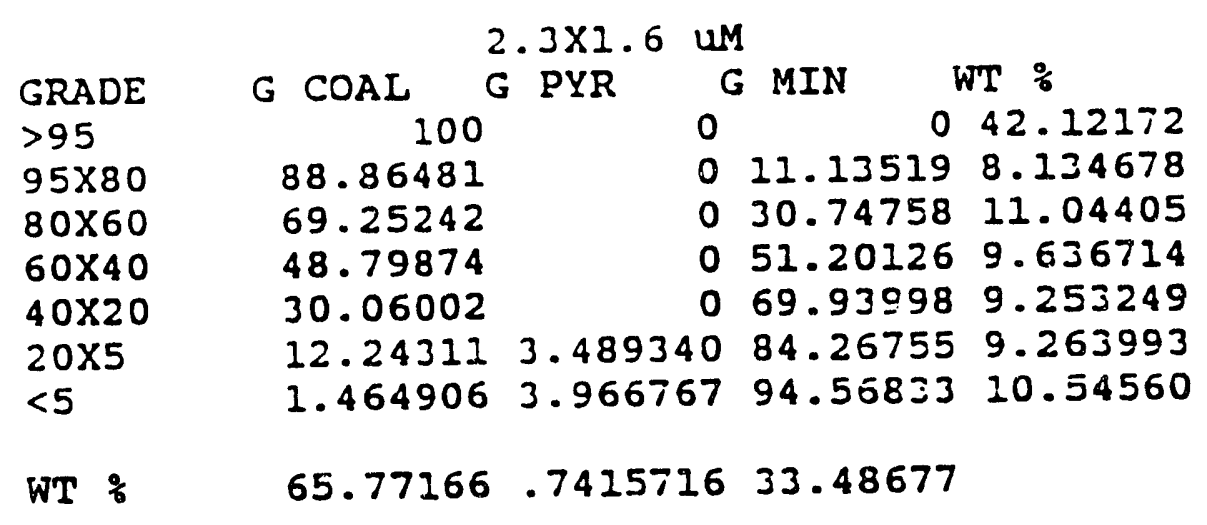


ELKHORN 5 MINUTE GRIND

\begin{tabular}{lrrrr}
\multicolumn{5}{c}{$9.3 \times 6.6$} \\
GRADE & G COAL & G PYR & G MIN & Wt\% \\
$>95$ & 98.67883 & 0 & 1.321171 & 48.25271 \\
$95 \times 80$ & 91.79101 & 2.188501 & 6.020486 & 33.50807 \\
$80 \times 60$ & 75.20465 & 0 & 24.79535 & 18.23922 \\
$60 \times 40$ & 0 & 0 & 0 & 0 \\
$40 \times 20$ & 0 & 0 & 0 & 0 \\
$20 \times 5$ & 0 & 0 & 0 & 0 \\
$<5$ & 0 & 0 & 0 & 0
\end{tabular}

WT $92.08935 \quad .7333243 \quad 7.177330$

\begin{tabular}{|c|c|c|c|c|}
\hline & & $6.6 \times 4.6 u$ & $u M$ & \\
\hline GRADE & G COAL & G PYR & G MIN & Wt\% \\
\hline$>95$ & 98.93356 & 0 & 01.066442 & 58.71485 \\
\hline $95 \times 80$ & 90.407 .34 & & 09.592657 & 35.78402 \\
\hline $80 \times 60$ & 76.93867 & & 023.06133 & 5.501131 \\
\hline $60 \times 40$ & 0 & & 0 & 0 \\
\hline $40 \times 20$ & 0 & 0 & 0 & 0 \\
\hline $20 \times 5$ & 0 & 0 & 0 & 0 \\
\hline$<5$ & 0 & 0 & 0 & 0 \\
\hline WT $\%$ & 94.67257 & 0 & $0 \quad 5.327432$ & \\
\hline FDגב & & $4.6 \times 3.3 \mathrm{u}$ & $\stackrel{M}{M} M T N$ & \\
\hline 95 & 99.11311 & G PYR & $\begin{array}{l}G 1 N \\
0.8868881\end{array}$ & $\begin{array}{l}\text { WE: } \\
42.27948\end{array}$ \\
\hline $95 \times 80$ & 88.77523 & 0 & 011.22477 & 14.13926 \\
\hline $80 \times 60$ & 68.27116 & 0 & 031.72384 & 13.64421 \\
\hline $60 \times 40$ & 52.23159 & 5259 & $\Rightarrow 34.51553$ & 15.74247 \\
\hline $40 \times 20$ & 0 & 0 & 0 & 0 \\
\hline $20 \times 5$ & 14.79835 & 530.63974 & 454.56191 & 7.650963 \\
\hline$<5$ & 4.469264 & 94.47033 & 31.060402 & 6.493617 \\
\hline$\%$ & 73.46109 & 10.56506 & $5 \quad 25.97385$ & \\
\hline
\end{tabular}

\begin{tabular}{|c|c|c|c|c|}
\hline & & $3.2 \times 2.34$ & $M$ & \\
\hline $\begin{array}{l}\text { GRADE } \\
>95\end{array}$ & $\begin{array}{l}\text { G COAL } \\
99.09485\end{array}$ & G PYR & $\begin{array}{l}G M I N \\
.205 i 529\end{array}$ & $\begin{array}{l}w た \% \\
=5 . . \Xi 924\end{array}$ \\
\hline $95 \times 80$ & 87.92074 & c & $=2.07 \subseteq 26$ & $=\equiv . .=001$ \\
\hline $80 \times 60$ & 70.25422 & .6734349 & $=0.07 .: 25$ & $\Xi \therefore . \therefore-\Xi \equiv 0$ \\
\hline $0 \times 40$ & 52.18701 & 8.235772 & : $99.4 Z: こ Z$ & $=0.025 \equiv 7$ \\
\hline $0 \times 20$ & 32.28524 & 4.538206 & 63.17656 & $8.06 \div:=0$ \\
\hline $0 \times 5$ & 10.63920 & 75.24453 & 24.21625 & 3.320506 \\
\hline & 0 & $c$ & 0 & 0 \\
\hline$\%$ & 71.79768 & 9747 & 20.16257 & \\
\hline
\end{tabular}




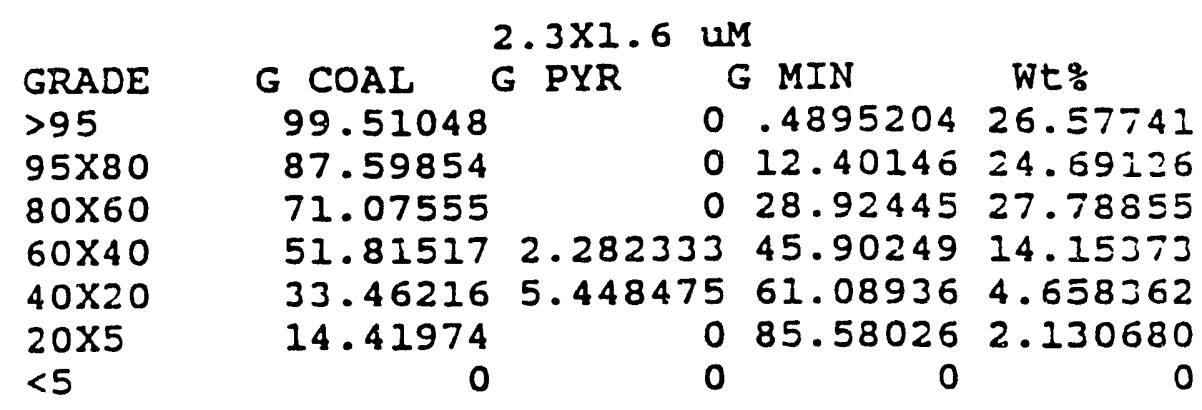

WT \&

$77.02717 \quad .5768449 \quad 22.39599$

\section{GRADE $>95$ \\ $95 \times 80$ \\ $80 \times 60$ \\ $60 \times 40$ \\ $40 \times 20$ \\ $20 \times 5$ \\ $<5$}

WT \%

GRADE
$>95$
$95 \times 80$
$80 \times 60$
$60 \times 40$
$40 \times 20$
$20 \times 5$
$<5$

WT $\div$

\begin{tabular}{|c|c|c|c|}
\hline COAI & $\begin{array}{l}1.6 \times 1.2 \\
\text { G PYR }\end{array}$ & G MIN & we \\
\hline 935 & & .2106454 & 3 \\
\hline & 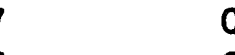 & 0023 & $1:$ \\
\hline & ; & 9425 & \\
\hline & . & 13084 & \\
\hline & & $\begin{array}{r}70483 \\
72305\end{array}$ & \\
\hline & & & \\
\hline
\end{tabular}

$75.44718 \quad .7593552 \quad 23.79347$

$<1.2 \mathrm{UM}$

$\begin{array}{rrrrr}\text { G COAL G PYR } & \text { G MIN } & \text { Wt: } \\ 100 & & 0 & 0 & 71.19258 \\ 86.57450 & & 0 & 13.42550 & 2.749356 \\ 69.77686 & & 0 & 30.22314 & 6.912541 \\ 49.35901 & & 0 & 50.64099 & 7.228997 \\ 29.61010 & .6523087 & 69.73760 & 3.475079 \\ 15.07369 & 29.25759 & 55.65872 & 3.540847 \\ 0 & 0 & 0 & 0\end{array}$

$84.95837 \quad 1.091254 \quad 13.95038$ 


$\begin{array}{lrrrrr}\text { GRADE } & \text { G COAL } & \text { G PYR } & \text { G MIN } & \text { Wt\% } \\ >95 & 98.03197 & 0 & 1.968032 & 48.04879 \\ 95 \times 80 & 90.77737 & 0 & 9.222628 & 12.46898 \\ 80 \times 60 & 60.04271 & 32.20176 & 7.755533 & 8.230049 \\ 60 \times 40 & 55.71936 & 0 & 44.28064 & 7.638098 \\ 40 \times 20 & 20.52167 & 41.99357 & 37.48476 & 11.86105 \\ 20 \times 5 & 7.945348 & 13.99362 & 78.06104 & 11.75303 \\ <5 & 0 & 0 & 0 & 0 \\ & & & & & \end{array}$

GRADE
$>95$
$95 \times 80$
$80 \times 60$
$60 \times 40$
$40 \times 20$
$20 \times 5$
$<5$

WI \%

GRADE $>95$

$95 \times 80$ $80 \times 60$ $60 \times 40$ $40 \times 20$ $20 \times 5$ $<5$ WI :

GRADE
$>95$
$95 \times 80$
$80 \times 60$
$60 \times 40$
$40 \times 20$
$20 \times 5$
$<5$

WT \%

\begin{tabular}{rrrrr}
\multicolumn{5}{c}{$6.6 \times 4.6$} \\
G COAL & G PYR & G MIN & Wt\% \\
98.59866 & & 0 & 1.401339 & 33.84195 \\
90.80208 & 1.706890 & 7.491030 & 28.30190 \\
74.44195 & 4.297803 & 21.26025 & 10.39773 \\
50.74924 & 27.20060 & 22.05015 & 13.02167 \\
36.57665 & 27.27497 & 36.14838 & 7.260327 \\
6.764710 & 91.95547 & 1.279825 & 7.176427 \\
0 & 0 & 0 & 0
\end{tabular}

$76.55614 \quad 13.05130 \quad 10.39256$
$80.63824 \quad 6.867418 \quad 12.49434$
Wt:

G MIN

01.398047

46.99367

1.6810107 .891360

6.00678226 .33168

15.3985037 .21370

$32.79346 \quad 27.27678 \quad 39.92976 \quad 9.3607 \geq 8$

$32.79346 \quad 27.27678 \quad 39.92976 \quad 9.3607 \geq 8$

19.4488780 .06892 .48221372 .308731

0

$3.3 \times 2.3 \mathrm{UM}$

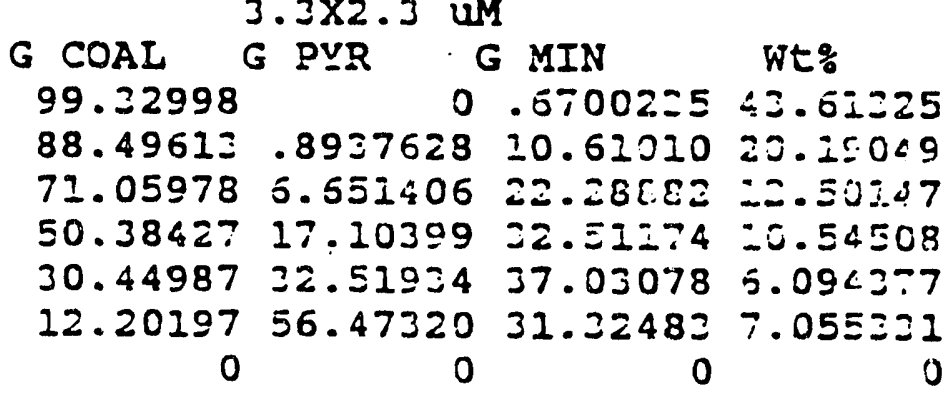

78.102038 .78183113 .11614 


\begin{tabular}{|c|c|c|c|c|}
\hline & & $2.3 \times 1.6 \mathrm{u}$ & & \\
\hline GRADE & G COAL & G PYR & G MIN & Wt\% \\
\hline$>95$ & 99.70971 & 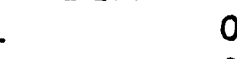 & .2902924 & 36.53508 \\
\hline $95 \times 80$ & 87.31568 & 0 & 12.68432 & 17.92503 \\
\hline $80 \times 60$ & 70.91286 & 2.905840 & 26.18130 & 14.09393 \\
\hline $60 \times 40$ & 49.61576 & 54.841694 & 45.54254 & 10.96029 \\
\hline $40 \times 20$ & 29.92287 & 26.62458 & 43.45254 & 7.720524 \\
\hline $20 \times 5$ & 12.68566 & 565.86740 & 21.44694 & 12.76515 \\
\hline$<5$ & 0 & c & 0 & \\
\hline VT $\%$ & 71.44237 & 711.40384 & 17.15379 & \\
\hline
\end{tabular}

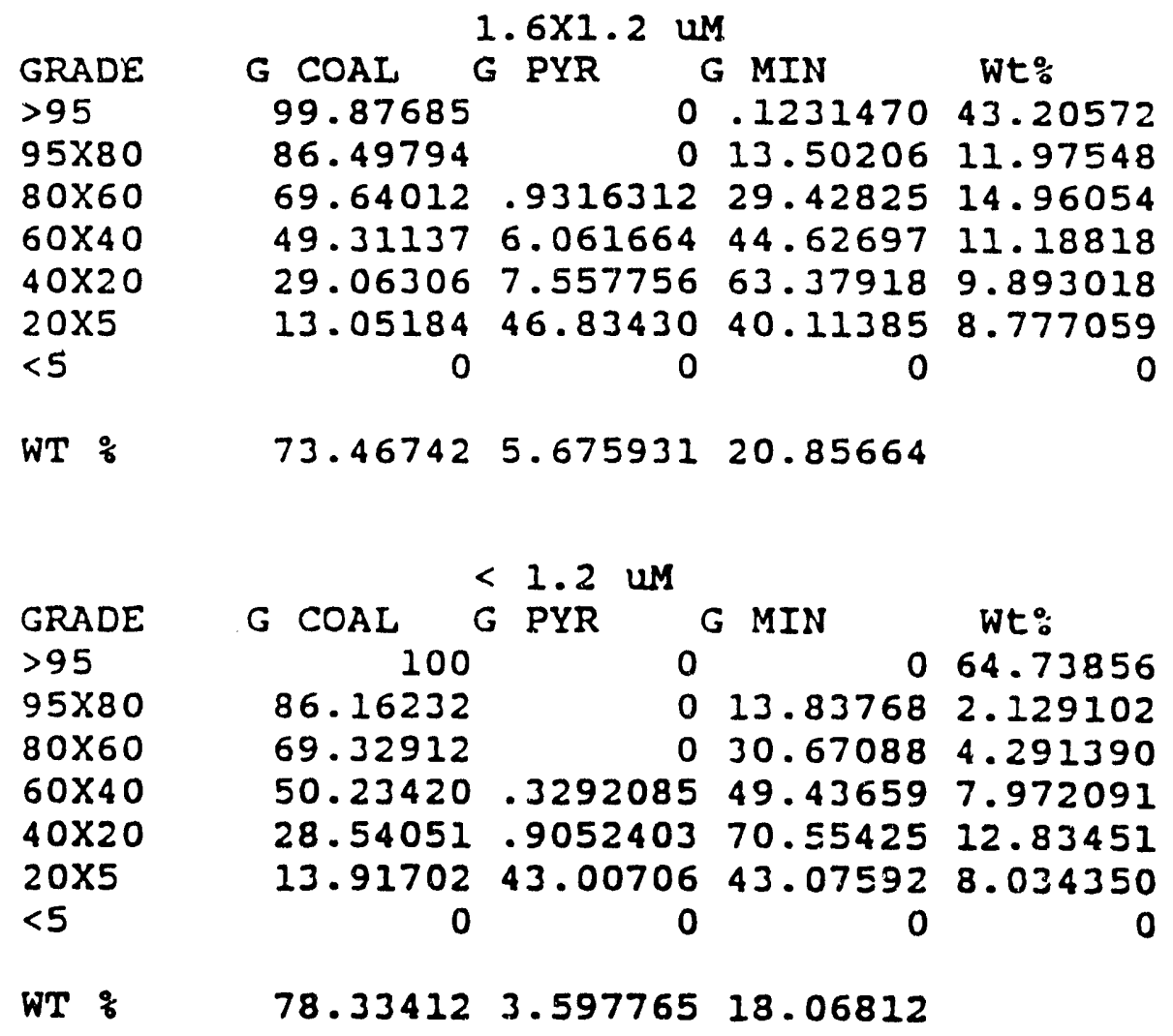


PITTSBURGH NO 8 FEED

$\begin{array}{cccccc}\text { GRADE } & \text { COAL } & +65 \text { MESH } \\ >95 & 97.8315 & .603663 & 1.56481 & 36.4431 \\ 95 \times 80 & 89.2306 & 4.95230 & 5.81706 & 24.0623 \\ 80 \times 60 & 71.1377 & 15.4093 & 13.4530 & 15.4202 \\ 60 \times 40 & 50.9560 & 27.1294 & 21.9147 & 7.09161 \\ 40 \times 20 & 32.2615 & 35.5894 & 32.1491 & 4.47900 \\ 20 \times 5 & 11.8292 & 56.6801 & 31.4906 & 1.68299 \\ <5 & 1.54558 & 74.9188 & 23.5356 & .815126\end{array}$

WT $8 \quad 82.29229 .365648 .34218$

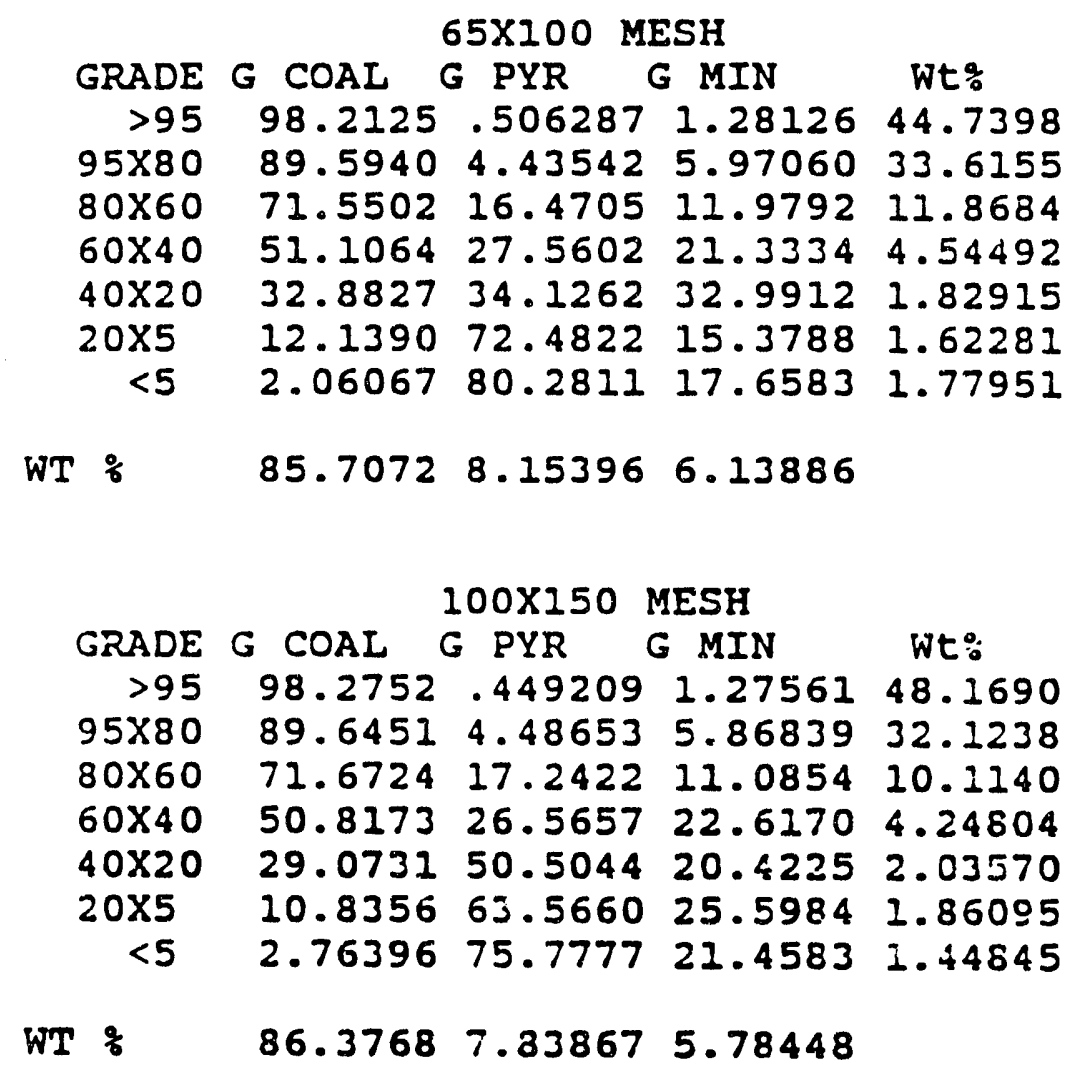




\begin{tabular}{|c|c|c|c|c|c|}
\hline \multicolumn{6}{|c|}{ PITTSBURGH NO 8 FEED } \\
\hline & RADE & G COAL & $\begin{array}{l}150 \times 200 \mathrm{M} \\
\text { G PYR }\end{array}$ & $\begin{array}{l}\text { MESH } \\
\text { G MIN }\end{array}$ & Wt\% \\
\hline & $>95$ & 98.50553 & .3150088 & 81.179459 & 52.76781 \\
\hline & $5 \times 80$ & 89.16154 & 4.946467 & 75.891988 & 26.22227 \\
\hline & $0 \times 60$ & 71.55118 & 317.67421 & 110.77461 & 8.270744 \\
\hline & $30 \times 40$ & 49.96747 & 730.63326 & $6 \quad 19.39927$ & 5.420991 \\
\hline & $0 \times 20$ & 30.66641 & 47.82066 & $6 \quad 21.51292$ & 2.481172 \\
\hline & $0 \times 5$ & 11.20249 & 68.33080 & 020.46671 & 2.388194 \\
\hline & $<5$ & 2.051790 & 86.27051 & 111.67770 & 2.428816 \\
\hline WT & $\%$ & 85.07811 & 19.503009 & 95.418877 & \\
\hline & & & $200 \times 270 \mathrm{M}$ & MESH & \\
\hline & RADE & G COAL & G PYR & G MIN & Wt: \\
\hline & $>95$ & 98.81300 & .1644143 & 31.022585 & 56.87920 \\
\hline & $5 \times 80$ & 89.92588 & 32.980047 & 7. 7.094071 & 21.47031 \\
\hline & $0 \times 60$ & 72.62954 & 414.30534 & 413.06512 & 10.04061 \\
\hline & $0 \times 40$ & 50.67964 & 428.22049 & 921.09987 & 4.094479 \\
\hline & $0 \times 20$ & 29.93942 & 246.02522 & 224.03536 & 3.655257 \\
\hline & $0 \times 5$ & 9.978290 & 55.16472 & 234.85699 & 2.479790 \\
\hline & $<5$ & 2.950034 & 480.38292 & 21.6 .66705 & 1.380361 \\
\hline WT & $\%$ & 86.26144 & 47.485052 & 26.253504 & \\
\hline & & & $270 \times 400 \mathrm{M}$ & MESH & \\
\hline & BRADE & G COAL & G PYR & G MIN & Wt: \\
\hline & $>95$ & 99.11975 & 5.0859022 & 2.7943511 & 60.25619 \\
\hline & $5 \times 80$ & 89.43363 & 32.800439 & 97.765932 & 17.96017 \\
\hline & $0 \times 60$ & 71.19708 & 814.61565 & $5 \quad 14.18727$ & 7.591229 \\
\hline & $0 \times 40$ & 50.30384 & 425.60336 & 624.09280 & $2.5=6758$ \\
\hline & $0 \times 20$ & 30.89317 & 746.32056 & 622.78627 & 4.010455 \\
\hline & $0 \times 5$ & 12.10952 & 272.27470 & D 15.61579 & $=.886061$ \\
\hline & $<5$ & 2.565571 & 188.59828 & 88.836149 & $2.7591=0$ \\
\hline N & 8 & 84.75240 & 09.680609 & 95.5 & \\
\hline
\end{tabular}


PITTSBURGH NO 8 FEED -400

GRADE
$>95$
$95 \times 80$
$80 \times 60$
$60 \times 40$
$40 \times 20$
$20 \times 5$
$<5$

WT $\%$
GRADE
$>95$
$95 \times 80$
$80 \times 60$
$60 \times 40$
$40 \times 20$
$20 \times 5$
$<5$

WT \%

GRADE
$>95$
$95 \times 80$
$80 \times 60$
$60 \times 40$
$40 \times 20$
$20 \times 5$
$<5$

WT \&

$\begin{array}{rrrrr}\text { G COAL } & 37 X 26 \text { UM PYR } & \text { G MIN } & \text { WT } \% \\ 97.91800 & .3409207 & 1.741079 & 42.15748 \\ 88.89138 & 3.317657 & 7.790965 & 27.32577 \\ 71.48631 & 4.658270 & 23.85542 & 6.153959 \\ 58.27031 & 18.70073 & 23.02896 & 4.655763 \\ 26.23041 & 68.14360 & 5.625987 & 3.720794 \\ 9.006922 & 39.76622 & 51.22686 & 15.47023 \\ 0 & 0 & 0 & 0\end{array}$

$75.51026 \quad 10.91193 \quad 13.57781$

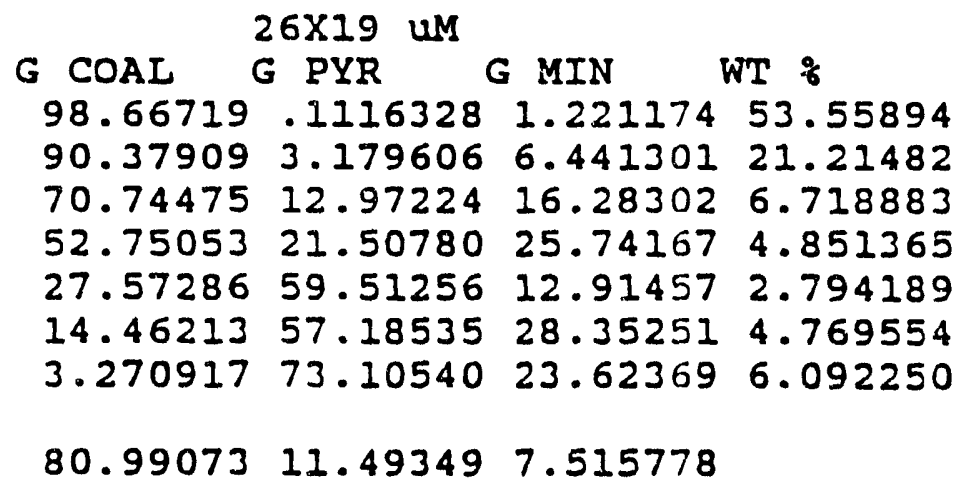

$19 \times 13$ UM

G COAL G PYR G MIN WT \% $98.86664 .1527522 \quad .9806037 \quad 55.11946$ $88.527492 .222627 \quad 9.249888 \quad 20.44652$ $\begin{array}{lllll}72.42669 & 4.547524 & 23.02579 & 6.622048\end{array}$ $51.97027 \quad 17.93524 \quad 30.09449 \quad 3.146150$ $27.94696 \quad 45.98369 \quad 26.069=5 \quad 5.967964$ $10.3903167 .5048722 .10482 \quad 5.792208$ $\begin{array}{lllll}1.839364 & 82.48186 & 15.67877 & 2.905546\end{array}$

$81.3498910 .45498 \quad 8.195125$ 


\begin{tabular}{|c|c|c|c|c|}
\hline & & 9.3 UM & & \\
\hline GRADE & G COAL & G PYR & ; MIN & WT \\
\hline$>95$ & 99.06414 & .1003358 & .8355267 & 54.66091 \\
\hline $95 \times 80$ & 89.12620 & 1.757550 & 9.116250 & 17.15538 \\
\hline $80 \times 60$ & 71.24575 & 57.685669 & 21.06858 & 8.730050 \\
\hline $60 \times 40$ & 50.22604 & 22.12539 & 27.64857 & 5.240182 \\
\hline $40 \times 20$ & 27.81945 & 544.64813 & 27.53241 & 6.206705 \\
\hline $20 \times 5$ & 10.81454 & 57.83795 & 31.34751 & .6 .613690 \\
\hline$<5$ & 3.149546 & 567.32222 & 29.52823 & 1.293082 \\
\hline WT \% & 80.80148 & 89.698313 & 9.500207 & \\
\hline & & $9.3 \times 6.6 \mathrm{uM}$ & & \\
\hline GRADE & G COAL & G PYR & MIN & WT\% \\
\hline$>95$ & 99.44023 & 3.0427683 & .5170060 & 56.49114 \\
\hline $95 \times 80$ & 88.86840 & 01.153949 & 9.977648 & 13.11343 \\
\hline $80 \times 60$ & 71.54859 & 96.406544 & 22.04487 & 8.116834 \\
\hline $60 \times 40$ & 52.58164 & 417.57350 & 29.84486 & $; 3.965273$ \\
\hline $40 \times 20$ & 29.33721 & 134.77871 & 35.88407 & 7.070428 \\
\hline $20 \times 5$ & 12.39537 & 759.81472 & 27.78991 & 6.350121 \\
\hline$<5$ & 2.716378 & 891.93782 & 5.345806 & 4.892781 \\
\hline WT $\%$ & 78.71539 & $9 \quad 12.14796$ & 9.136659 & \\
\hline & & $6.6 \times 4.6 \mathrm{uM}$ & & \\
\hline GRADE & G COAL & G PYR & MIN & WT $\%$ \\
\hline$>95$ & 99.55294 & 4.0027455 & .4443163 & 60.49102 \\
\hline $95 \times 80$ & 89.33114 & 4.5860896 & 10.08277 & 10.66910 \\
\hline $80 \times 60$ & 70.03183 & 35.136160 & 24.83201 & 8.729596 \\
\hline $60 \times 40$ & 49.51627 & 76.279685 & 44.20404 & 6.201219 \\
\hline $40 \times 20$ & 28.98009 & $932.1=159$ & $38.908=1$ & 5.968798 \\
\hline $20 \times 5$ & 12.21996 & 669.18642 & 18.59362 & 4.585385 \\
\hline$<5$ & 1.731772 & 283.46663 & 14.80159 & $=.254877$ \\
\hline $\mathbf{T}$ & 21 & $770=7$ & 87 & \\
\hline
\end{tabular}


PITTSBURGH NO 8 FEED -400

\begin{tabular}{|c|c|c|c|c|}
\hline GRAL & G COAL & $\begin{array}{l}4.6 \times 3.3 u \\
\text { G PYR }\end{array}$ & G MIN & WT \% \\
\hline$>95$ & 99.75448 & 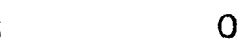 & .2455169 & 65.53099 \\
\hline $95 \times 80$ & 89.48507 & 1.430076 & 9.084853 & 8.666850 \\
\hline $80 \times 60$ & 69.55979 & 4.521258 & 25.91896 & 7.139578 \\
\hline $60 \times 40$ & 48.55302 & 8.747857 & 42.69912 & 4.458173 \\
\hline $40 \times 20$ & 30.01436 & 13.05198 & 56.93366 & 5.349554 \\
\hline $20 \times 5$ & 13.20724 & 53.75 & 33.04120 & 5.897002 \\
\hline$<5$ & 2.351509 & 70.48588 & 27.16261 & 2.957849 \\
\hline$T \%$ & 82.71051 & .789555 & ; 10.49993 & \\
\hline
\end{tabular}

\begin{tabular}{|c|c|c|c|c|}
\hline GRA & G COAL & $\begin{array}{l}3.3 \times 2.3 \\
G \text { PYR }\end{array}$ & $\stackrel{M}{M}$ MIN & WT \% \\
\hline$>95$ & 99.90612 & 0 & .0938817 & 62.79762 \\
\hline $95 \times 80$ & 88.75317 & .1925252 & 11.05431 & 7.252874 \\
\hline $80 \times 60$ & 69.14120 & 2.266920 & 28.59188 & 6.911635 \\
\hline $60 \times 40$ & 49.94599 & 13.60370 & 36.45031 & 5.881055 \\
\hline $40 \times 20$ & 29.42215 & 17.43724 & 53.14061 & 5.390547 \\
\hline $20 \times 5$ & 12.89052 & 43.85726 & 43.25222 & 6.069639 \\
\hline$<5$ & 1.210710 & 82.48602 & 16.30327 & 5.69662 \\
\hline 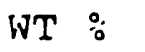 & 79.32936 & .271545 & 11.39910 & \\
\hline
\end{tabular}

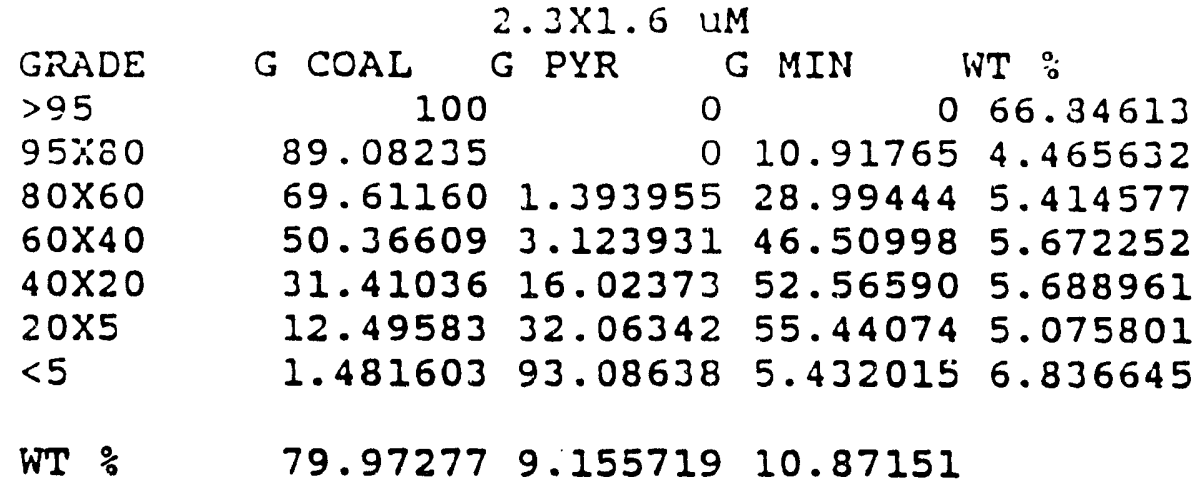


PITTSBURGH NO 85 MINUTE GRIND

\begin{tabular}{lrrrrr} 
& \multicolumn{4}{c}{$9.3 \times 6.6$} & UM \\
GRADE & G COAL & G & PYR & G MIN & Wt\% \\
$>95$ & 99.06859 & .3052587 & .6261514 & 40.31502 \\
$95 \times 80$ & 90.92900 & 5.848019 & 3.222983 & 59.68498 \\
$80 \times 60$ & 0 & 0 & 0 & 0 \\
$60 \times 40$ & 0 & 0 & 0 & 0 \\
$40 \times 20$ & 0 & 0 & 0 & 0 \\
$20 \times 5$ & 0 & 0 & 0 & 0 \\
$<5$ & 0 & 0 & 0 & 0
\end{tabular}

WT \%

$94.21048 \quad 3.613454 \quad 2.176070$

GRADE
$>95$
$95 \times 80$
$80 \times 60$
$60 \times 40$
$40 \times 20$
$20 \times 5$
$<5$

WT :

GRADE

$>05$

$95 \times 80$

$80 \times 60$

$60 \times 40$

$40 \times 20$

$20 \times 5$

$<5$

WT $\%$

GRADE

$>95$

$95 \times 80$

$80 \times 60$

$60 \times 40$

$40 \times 20$

$20 \times 5$

$<5$

WT \%
$6.6 \times 4.6 \mathrm{UM}$

G COAL

G PYR

G MIN

Wt:

$98.47334 .6230084 .9036536 \quad 41.59406$

$87.64937 \quad 8.150150 \quad 4.200483 \quad 33.64958$

$\begin{array}{lllll}67.54507 & 29.00133 & 3.453595 & 18.78683\end{array}$

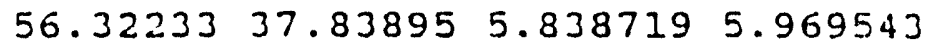

$\begin{array}{llll}0 & 0 & 0 & 0 \\ 0 & 0 & 0 & 0 \\ 0 & 0 & 0 & 0\end{array}$

$86.50446 \quad 10.70887 \quad 2.786677$

$4.6 \times 2.2$ UM

$G$ COAL G PYR G MIN Wt:

$99.20642 \quad 0468396 \quad .746738445 .47103$

$88.79415 \quad 4.0546117 .15124215 .45588$

$\begin{array}{lllll}70.69107 & 23.28053 & 6.028399 & 8.231250\end{array}$

$\begin{array}{lllll}50.46283 & 39.48231 & 10.05486 & 7.989818\end{array}$

$29.85601 \quad 59.34736 \quad 10.79664 \quad 11.66818$

$\begin{array}{lllll}14.92256 & 84.90085 & .1765924 & 1.773952\end{array}$

$.9378797 \quad 93.63821 \quad 5.423914 \quad 9.409893$

$72.52137 \quad 22.96093 \quad 4.517703$

$3.3 \times 2.3 \mathrm{UM}$

G COAL G PYR G MIN Wt:

$\begin{array}{llllll}99.33273 & .0368536 & .6304208 & 44.30417\end{array}$

$87.43270 \quad 4.962745 \quad 7.604556 \quad 17.63637$

$\begin{array}{lllll}70.06138 & 17.39782 & 12.54080 & 9.498947\end{array}$

$52.31554 \quad 42.13676 \quad 5.547700 \quad 9.210359$

$28.19263 \quad 53.76987 \quad 18.03749 \quad 5.631090$

$10.62796 \quad 86.71282 \quad 2.659220 \quad 5.578316$

$2.04557981 .23864 \quad 16.71578 \quad 8.140743$

$73.24898 \quad 20.90351 \quad 5.847514$ 


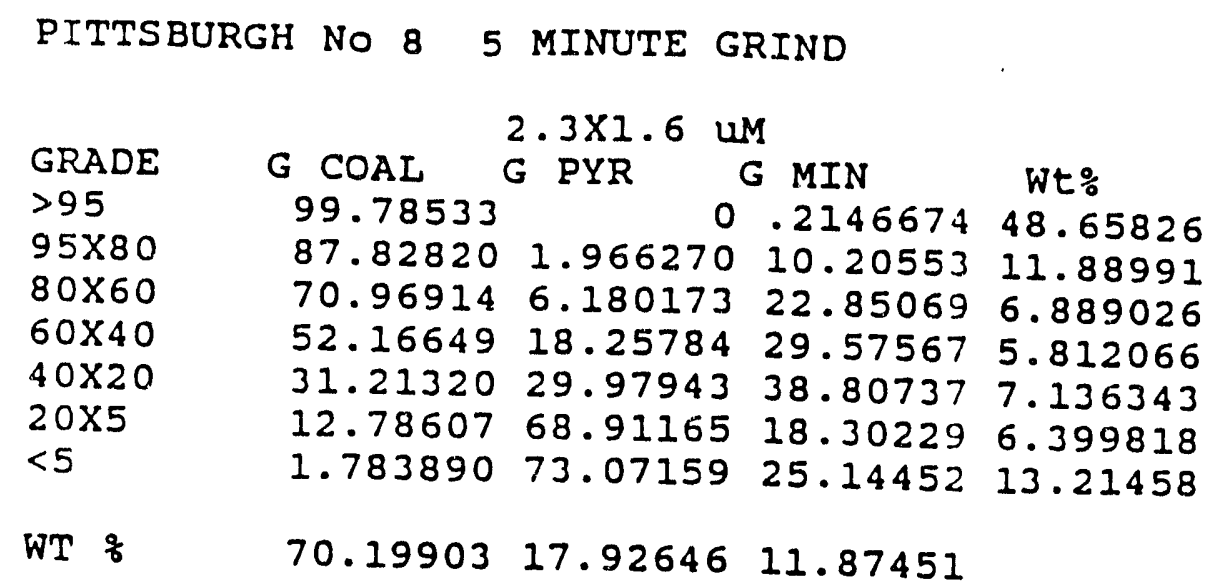

\begin{tabular}{|c|c|c|c|c|}
\hline & & $1.6 \times 1.2 u$ & & \\
\hline $\begin{array}{l}\text { GRADE } \\
\end{array}$ & G COAL & G PYR & G MIN & Wt: \\
\hline $\begin{array}{l}>95 \\
95 \times 80\end{array}$ & 99.89387 & 0 & .1061319 & 45.56763 \\
\hline $80 \times 60$ & $\begin{array}{l}87.81165 \\
69.93942\end{array}$ & .9010699 & 11.28728 & 5.191519 \\
\hline $60 \times 40$ & 51.34672 & $\begin{array}{l}3.457376 \\
9.947684\end{array}$ & 26.60321 & 6.043699 \\
\hline $40 \times 20$ & 28.70015 & 34.24012 & $\begin{array}{l}38.71159 \\
37.05973\end{array}$ & 7.844478 \\
\hline $20 \times 5$ & 13.18136 & 56.44191 & 30.37673 & $\begin{array}{r}12.61157 \\
170077\end{array}$ \\
\hline$<5$ & .5978635 & 90.99692 & 8.405217 & 10.82927 \\
\hline & 63.57687 & 95992 & 46 & \\
\hline
\end{tabular}

\begin{tabular}{|c|c|c|c|c|}
\hline GRADE & G COAL & $\begin{array}{lll}< & 1.2 \\
G & \text { PYR }\end{array}$ & G MIII & Wt: \\
\hline$>95$ & 100 & 0 & 0 & 61.46956 \\
\hline $95 \times 80$ & 85.30349 & .7762083 & 13.92031 & 1.078291 \\
\hline $\begin{array}{l}80 \times 60 \\
60 \times 40\end{array}$ & 68.50932 & 2.810165 & 28.68052 & 3.112051 \\
\hline $\begin{array}{l}60 \times 40 \\
40 \times 20\end{array}$ & 49.43603 & 7.002179 & 43.56179 & 4.808019 \\
\hline $20 \times 5$ & $\begin{array}{l}28.41233 \\
10.39211\end{array}$ & $\begin{array}{l}11.74449 \\
24.47642\end{array}$ & $\begin{array}{l}59.84319 \\
65.13147\end{array}$ & 5.085370 \\
\hline$<5$ & .4198272 & 90.66754 & $\begin{array}{l}65.13147 \\
8.912637\end{array}$ & $\begin{array}{l}4.572696 \\
19.87401\end{array}$ \\
\hline NT \% & 68.90183 & 20.16825 & 10.92992 & \\
\hline
\end{tabular}




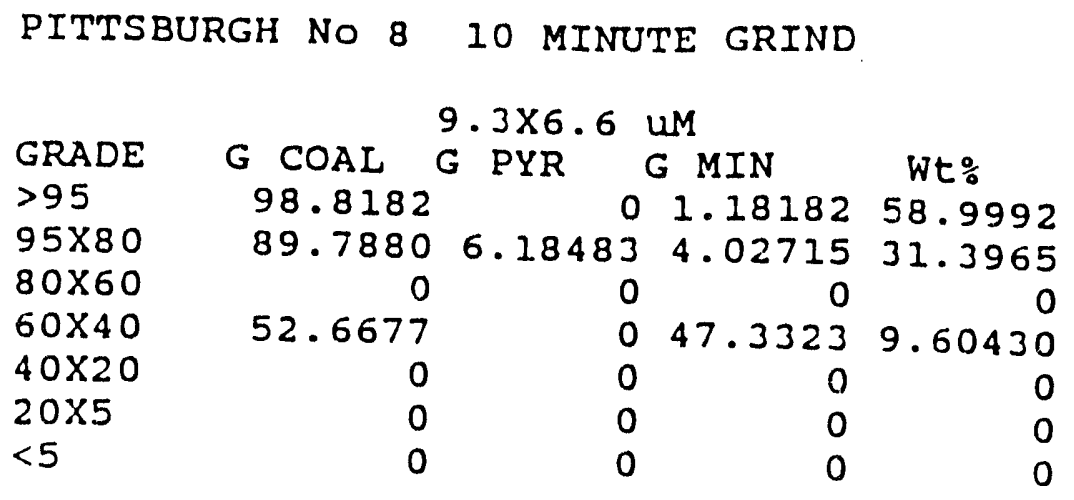

WT \% $91.5506 \quad 1.94182 \quad 6.50759$

\begin{tabular}{|c|c|c|c|c|}
\hline & & $6.6 \times 4.64$ & UM & \\
\hline $\begin{array}{l}\text { GRADE } \\
>95\end{array}$ & $\begin{array}{c}\text { G COAL } \\
98.8921\end{array}$ & $\begin{array}{l}G \text { PYR } \\
200972\end{array}$ & G MIN & Wt\% \\
\hline $95 \times 80$ & 87.6994 & 6.50889 & $\begin{array}{r}906912 \\
5 \quad 79170\end{array}$ & $\begin{array}{l}57.5689 \\
17.8871\end{array}$ \\
\hline $80 \times 60$ & 71.9197 & 17.4725 & 10.6078 & $\begin{array}{l}17.8871 \\
10.7689\end{array}$ \\
\hline $60 \times 40$ & 49.2081 & 24.1429 & 26.6490 & 6.39924 \\
\hline $40 \times 20$ & 32.3350 & 58.0190 & 9.64602 & 4.21757 \\
\hline $20 \times 5$ & 10.4851 & 84.6333 & 4.88154 & 3.15825 \\
\hline$<5$ & 0 & 0 & 0 & \\
\hline WT $\stackrel{\circ}{\circ}$ & 85.2068 & 9.82643 & 4.96674 & \\
\hline & & $4.6 \times 2.34$ & UM & \\
\hline GNADE & $G$ COAL & G PYR & G MIN & Wt: \\
\hline$>95$ & 99.4155 & 0 & .584456 & 62.9342 \\
\hline $95 \times 80$ & 89.1371 & 2.88416 & 7.97870 & 16.7114 \\
\hline $80 \times 60$ & 71.9551 & 13.1887 & 14.8562 & 4.73667 \\
\hline $60 \times 40$ & 51.3898 & 33.1659 & 15.4443 & 10.6205 \\
\hline $40 \times 20$ & 23.1105 & 60.0096 & 16.8799 & 2.77612 \\
\hline $20 \times 5$ & 0 & 0 & 0 & 0 \\
\hline$<5$ & 782 & 95.0277 & .304437 & 2.22112 \\
\hline WT $\%$ & 87.0738 & 8.4 & 4.52049 & \\
\hline
\end{tabular}

\begin{tabular}{|c|c|c|c|c|}
\hline & & $3.3 \times 2.3$ & UM & \\
\hline GRA & G COAL & G PYR & G MIN & Wt: \\
\hline & 99.5848 & 0 & .415231 & 62.4453 \\
\hline $95 \times 80$ & 89.5053 & 2.07750 & 8.41724 & 8.49536 \\
\hline $80 \times 60$ & 67.0585 & 12.9330 & 20.0084 & 9.52459 \\
\hline $60 \times 40$ & 49.6235 & 34.1640 & 16.2124 & 5.75191 \\
\hline $40 \times 20$ & 30.5604 & 60.2686 & 9.17103 & 7.54523 \\
\hline $20 \times 5$ & 8.51314 & 78.1741 & 13.3127 & 4.91520 \\
\hline$<5$ & .517103 & 99.2949 & .188034 & 1.32244 \\
\hline$\%$ & 81.7622 & 13.0763 & 6142 & \\
\hline
\end{tabular}




\begin{tabular}{|c|c|c|c|c|}
\hline GRADE & G COAL & $\begin{array}{l}2.3 \times 1.6 \\
\text { G PYR }\end{array}$ & $\underset{G}{U M I N}$ & Wt: \\
\hline$>95$ & 99.85722 & & 0.1427812 & 58.35895 \\
\hline $\begin{array}{l}95 \times 80 \\
80 \times 60\end{array}$ & 87.82213 & 11.89829 & 610.27959 & 4.967360 \\
\hline $60 \times 40$ & 49.91181 & 18.2112 & $\begin{array}{ll}822.43587 \\
9 & 31.87691\end{array}$ & 12.41016 \\
\hline $40 \times 20$ & 26.99108 & $358.4098=$ & 514.59907 & $\begin{array}{l}4.933839 \\
4.696969\end{array}$ \\
\hline $20 \times 5$ & 10.78123 & 369.54467 & 719.67410 & 4.506630 \\
\hline$<5$ & 1.808290 & 92.31768 & 85.874034 & 10.12610 \\
\hline WT $q$ & 75.88982 & ? 16.99190 & 7.118195 & \\
\hline
\end{tabular}

\begin{tabular}{|c|c|c|c|c|}
\hline & & $1.6 \times 1.2$ & UM & \\
\hline GRADE & G COAL & G PYR & G MIN & Wt\% \\
\hline $\begin{array}{l}>95 \\
05 Y 90\end{array}$ & 99.91359 & & 0.0864102 & 47.23537 \\
\hline & 87.97239 & & 012.02761 & 6.279508 \\
\hline $80 \times 60$ & 69.47388 & 1.47177 & $4 \quad 29.05434$ & 7.553750 \\
\hline $60 \times 40$ & 51.46029 & 19.4515 & 629.08815 & 3.987659 \\
\hline $40 \times 20$ & 30.92314 & 42.4962 & 126.58065 & 5.557874 \\
\hline $20 \times 5$ & 12.43383 & 80.4319 & 07.134278 & 10.25578 \\
\hline$<5$ & .7007967 & 96.2564 & 93.042710 & 19.13006 \\
\hline $\mathrm{VT}$ & 63.14665 & $29.9115^{\circ}$ & 76.941786 & \\
\hline
\end{tabular}

\begin{tabular}{|c|c|c|c|c|}
\hline GRIDE & G COAL & $\begin{array}{ll}< & 1.2 \\
G & \text { PYR }\end{array}$ & G MIN & Wt: \\
\hline$>95$ & 99.99617 & 0 & .0038279 & 51.18052 \\
\hline $95 \times 80$ & 87.16284 & 0 & 12.83716 & 1.216295 \\
\hline $80 \times 60$ & 68.29898 & 0 & 31.70102 & 2.415400 \\
\hline $60 \times 40$ & 49.34940 & 4.790842 & 45.85976 & 2.377574 \\
\hline $40 \times 20$ & 28.14550 & 15.64353 & 56.21097 & 3.842493 \\
\hline $20 \times 5$ & 10.24538 & 24.99283 & 64.76179 & 6.954749 \\
\hline$<5$ & .3114796 & 7893 & 23.30959 & 32.01297 \\
\hline WT \% & 56.95547 & 6 & 16.14017 & \\
\hline
\end{tabular}


SAMPLE: Elk/S oin

ASSAY: ASH

\begin{tabular}{|c|c|c|c|c|c|c|c|c|c|c|}
\hline S6 & & MIVIDU & & cunt & LATIVE & FLOAT & CU:H & LATIVI & SIMK & \\
\hline SHI FLI & IT & ASSAY & PROB & זע & PROO & ASSAY & עT & PROS & Assar & \\
\hline$\cdots$ & 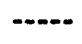 & 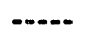 & $\cdots$ & 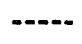 & 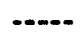 & 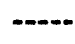 & 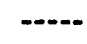 & - & & \\
\hline 1.20 & 04.13 & 3.90 & 1.72 & 14.13 & 1.72 & 3.90 & 100.00 & 25.13 & 23.13 & \\
\hline .201 .40 & 26.02 & 3.78 & 0.98 & 70.15 & 2.70 & 3.86 & 35.87 & 23.71 & $\$ 2.41$ & \\
\hline 1.401 .60 & 4.34 & 39.47 & 1.54 & 74.19 & 4.24 & 3.70 & 29.85 & 22.73 & 76.14 & 2.32 \\
\hline 1.601 .70 & 2.53 & 67.85 & 1.72 & 717.02 & 3.96 & 7.71 & 23.51 & 21.19 & 83.06 & 5.7 \\
\hline 1.70 & 22.98 & 84.73 & 19.17 & 100.00 & 25.43 & 25.13 & 22.98 & 19.17 & 84.73 & \\
\hline
\end{tabular}

SAIPLE: EIK/S a In

ASSAY: SULLFUR

\begin{tabular}{|c|c|c|c|c|c|c|c|c|c|c|}
\hline 56 & & AoIVIM & & cunt & LIIVE & FLOAI & cuno & MIIU & SIMK & \\
\hline SNI FLI & זע & ASSAY & PROD & II & PROS & Assar & in & Pros & ASSAY & $l$ \\
\hline -- -..- & $-\cdots$ & -... & $\ldots$ & $\ldots$ & $\ldots$ & $\ldots$ & .... & $\cdots$ & - & \\
\hline 1.20 & 44.13 & 0.97 & 0.13 & 14.13 & 0.13 & 0.97 & 100.00 & 1.63 & 1.63 & 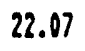 \\
\hline .201 .40 & 26.02 & 1.02 & 0.27 & 70.15 & 0.69 & 0.99 & 55.87 & 1.20 & 2.14 & 37. \\
\hline 401.60 & 4.34 & 1.50 & 0.07 & 74.19 & 0.76 & 1.02 & 29.85 & 0.93 & 3.12 & 72. \\
\hline 1.601 .70 & 2.53 & 2.11 & 0.05 & 77.02 & 0.81 & 1.03 & 25.31 & 0.81 & 3.10 & 73. \\
\hline 1.70 & 22.98 & 3.54 & 0.81 & 100.00 & 1.63 & 1.63 & 22.98 & 0.81 & 3.34 & 88. \\
\hline
\end{tabular}

:AHPLE: EIK/S an

ASSAY: PYRITIC

\begin{tabular}{|c|c|c|c|c|c|c|c|c|c|c|}
\hline S6 & & Divide & & cunt & AlIVE & FLOAI & cuni & AIIVE & SIIII & \\
\hline SHI FLI & עI & ASSAY & PROB & VI & Pros & ASSAY & vi & PPOS & ASSAY & 1 \\
\hline$\cdots$ & $\cdots \cdot-$ & $\cdots$ & $\cdots$ & 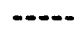 & $\cdots$ & $\cdots$ & $\cdots$ & 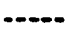 & 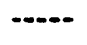 & 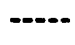 \\
\hline 1.20 & 44.13 & 0.08 & 0.04 & 14.13 & 0.04 & 0.08 & 100.00 & 1.00 & 1.08 & 22.07 \\
\hline 1.201 .40 & 26.02 & 0.18 & 0.05 & 70.15 & 0.08 & 1.12 & 55.87 & 1.04 & 1.86 & 37.14 \\
\hline 1.401 .60 & 4.34 & 1.03 & 0.05 & 74.49 & 0.13 & 0.17 & 29.85 & 0.99 & 3.33 & 72.32 \\
\hline$\therefore .501 .70$ & 2.53 & 2.04 & 0.05 & 77.02 & 0.18 & 0.23 & 25.51 & 0.93 & 3.72 & 79.76 \\
\hline. .70 & 29.98 & 3.90 & 0.96 & 100.00 & 1.08 & 1.08 & 22.98 & 0.90 & 3.10 & 88.51 \\
\hline
\end{tabular}




\begin{tabular}{|c|c|c|c|c|c|c|}
\hline Saple & Veight & Ash & Sulfur & Mineral & Rec & Rej \\
\hline \multirow[t]{5}{*}{ S ain } & 14.13 & 3.9 & .97 & \multicolumn{3}{|c|}{4.745558 .6771992 .61593} \\
\hline & 70.19 & 3.86 & .99 & \multicolumn{3}{|c|}{4.713393 .3060688 .34177} \\
\hline & 74.19 & 3.7 & 1.02 & \multicolumn{3}{|c|}{6.71796 .9952382 .35770} \\
\hline & 77.02 & 7.74 & 1.05 & \multicolumn{3}{|c|}{0.936787 .9031875 .73051} \\
\hline & 100 & 25.43 & 1.63 & 28.3609 & 100 & 0 \\
\hline \multirow[t]{5}{*}{10 in } & 26.21 & 2.86 & .72 & \multicolumn{3}{|c|}{3.184834 .1121396 .46567} \\
\hline & 69.11 & 3.94 & .87 & \multicolumn{3}{|c|}{4.733788 .7822887 .34087} \\
\hline & 75.04 & 5.03 & .8 & \multicolumn{3}{|c|}{3.94995 .1705082 .72576} \\
\hline & 82.05 & 8.94 & .96 & \multicolumn{3}{|c|}{10.183299 .3761767 .668557} \\
\hline & 100 & 23.19 & 1.45 & 25.8427 & 100 & 0 \\
\hline \multirow[t]{5}{*}{30 ain } & 2.52 & 19.33 & 1.23 & \multicolumn{3}{|c|}{21.55292 .74252198 .05453} \\
\hline & 37.09 & 7.79 & 1.16 & \multicolumn{3}{|c|}{9.051246 .7979087 .97513} \\
\hline & 78.49 & 9.82 & 1.16 & \multicolumn{3}{|c|}{11.243696 .6466068 .38909} \\
\hline & 85.11 & 13.68 & 1.23 & \multicolumn{3}{|c|}{15.450999 .8302352 .89667} \\
\hline & 100 & 25.03 & 1.61 & 27.9179 & 100 & 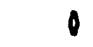 \\
\hline
\end{tabular}

PITTSRURgh WO. 8 SEAK

\begin{tabular}{|c|c|c|c|c|c|c|}
\hline Sapple & Veight & Ast & Sulfur & Mineral & Rec & Rej \\
\hline \multirow[t]{5}{*}{ s cin } & 37.15 & 3.49 & 3.29 & \multicolumn{3}{|c|}{3.578742 .1778687 .68890} \\
\hline & 80.93 & 3.48 & 3.2 & \multicolumn{3}{|c|}{3.518491 .9417073 .47059} \\
\hline & 86.07 & 4.09 & 3.23 & \multicolumn{3}{|c|}{6.204797 .0708168 .27676} \\
\hline & 89.96 & 6.29 & 3.13 & \multicolumn{3}{|c|}{8.679790 .7908053 .61697} \\
\hline & 100 & 13.26 & 1.57 & 16.8343 & 100 & \\
\hline \multirow[t]{5}{*}{$10 \mathrm{na}$} & 31.73 & 3.96 & 3.3 & \multicolumn{3}{|c|}{6.091835 .3055587 .61126} \\
\hline & 82.35 & 3.73 & 3.27 & \multicolumn{3}{|c|}{$3.826991 .8882269 .219 i^{\circ}$} \\
\hline & 87.61 & 1.49 & 3.36 & \multicolumn{3}{|c|}{6.697296 .8510162 .39390} \\
\hline & 90.12 & 3.68 & 3.5 & \multicolumn{3}{|c|}{8.059499 .0458253 .03509} \\
\hline & 100 & 12.16 & 4.49 & 15.6023 & 100 & \\
\hline \multirow[t]{5}{*}{30 ain } & 18.31 & 4.59 & 3.73 & \multicolumn{3}{|c|}{7.008720 .5162692 .45508} \\
\hline & 60.85 & 1.26 & 3.47 & \multirow{2}{*}{\multicolumn{3}{|c|}{$\begin{array}{l}6.509368 .5412676 .71245 \\
8.186197 .1546357 .60325\end{array}$}} \\
\hline & 80.09 & 3.67 & 3.75 & & & \\
\hline & 92.02 & 7.59 & 3.13 & \multicolumn{3}{|c|}{10.350799 .3934613 .95765} \\
\hline & 100 & 13.34 & 4.73 & 17.0089 & 100 & \\
\hline
\end{tabular}




\begin{tabular}{|c|c|c|c|c|c|c|c|c|c|c|}
\hline aple & Veight & Hiseral & Carbon & H-Hait & C-Vait & $\begin{array}{l}\text { Cun } \\
\text { Yeight }\end{array}$ & $\begin{array}{c}\text { Cua } \\
H \text {-Unil }\end{array}$ & $\begin{array}{r}\text { Cue } \\
\text { C-Uail }\end{array}$ & $\begin{array}{c}\text { Cun } \\
\text { C-Rec }\end{array}$ & $\begin{array}{l}\text { Cus } \\
\text { n-Res }\end{array}$ \\
\hline 5 & 45.6 & 2.2 & 97.5 & 114 & 1446 & 45.6 & 114 & \multirow{2}{*}{\multicolumn{3}{|c|}{$\begin{array}{r}444656.32090 \quad 94.60980 \\
038.576 .4943283 .85305\end{array}$}} \\
\hline & 18.2 & 12.5 & 87.3 & 227.5 & 1992.5 & 63.8 & 341.5 & & & \\
\hline & 16.3 & 30 & 70 & 489 & $\|1\|$ & 80.1 & 830.5 & \multicolumn{3}{|c|}{7179.590 .9482560 .73193} \\
\hline & 10.1 & 50 & so & 305 & 505 & 90.2 & 1335.5 & \multicolumn{3}{|c|}{7684.597 .3434736 .85430} \\
\hline & 5.31 & 70 & 30 & 371.7 & 159.3 & 95.51 & 1707.2 & \multicolumn{3}{|c|}{7843.899 .3634419 .27942} \\
\hline & 3.88 & 87.5 & 12.5 & 339.5 & 48.5 & 99.39 & 2046.7 & \multicolumn{3}{|c|}{7992.399 .977833 .227027} \\
\hline & .7 & 97.5 & 2.5 & 68.25 & 1.75 & 100.09 & 2114.95 & 7894.05 & 100 & \\
\hline . & 46.2 & 2.5 & 97.5 & 115.5 & 304.5 & 46.2 & 115.5 & \multicolumn{3}{|c|}{4904.559 .7261395 .30302} \\
\hline & 16.4 & 12.5 & 87.3 & 203 & 1435 & 62.6 & 320.5 & \multicolumn{3}{|c|}{5939.578 .7531086 .97194} \\
\hline & 10.4 & 30 & 70 & 312 & 728 & 73 & 632.5 & \multicolumn{3}{|c|}{6667.504 .4058174 .28940} \\
\hline & 9.99 & 30 & 50 & 499.5 & 499.5 & 82.99 & 1132 & \multicolumn{3}{|c|}{716795.0287953 .98314} \\
\hline & 9.26 & 70 & 30 & 648.2 & 277.8 & 92.25 & 1760.2 & \multicolumn{2}{|c|}{7444.898 .71220} & 27.636 ? \\
\hline & 7.77 & 87.5 & 12.5 & 9.875 & 97.125 & \multicolumn{2}{|c|}{100.022960 .073} & 7541.925 & 100 & \\
\hline & 0 & 97.9 & 2.5 & 0 & 0 & \multicolumn{3}{|c|}{100.022460 .0737541 .923} & 100 & \\
\hline 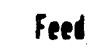 & 31.1 & 2.3 & $\pi . d$ & 95.23 & & 3 & 95.25 & \multicolumn{3}{|c|}{3714.7555 .9296197 .19946} \\
\hline & 16.7 & 12.3 & 87.5 & 201.75 & 1461.25 & 34.8 & $3 M$ & \multicolumn{3}{|c|}{517677.9289290 .93092} \\
\hline & 8.89 & 30 & 70 & 266.7 & 622.3 & 63.69 & 570.7 & \multicolumn{3}{|c|}{5798.387 .2941682 .97460} \\
\hline & 7.79 & 50 & 50 & 389.5 & 399.5 & 71.48 & 960.2 & \multicolumn{3}{|c|}{6187.893 .1624071 .35484} \\
\hline & 10 & 70 & 30 & 700 & 300 & 81.48 & 1660.2 & \multicolumn{3}{|c|}{6487.897 .6791530 .17210} \\
\hline & 10.8 & 87.5 & 12.5 & 945 & 135 & $9 ?: ? 8$ & 2605.2 & \multicolumn{3}{|c|}{6622.899 .7116822 .28040} \\
\hline & 7.66 & 97.3 & 2.5 & 746.05 & 19.15 & 99.84 & 3332.05 & 6641.95 & 100 & \\
\hline
\end{tabular}

PITTSuren 以. 1

Sasple Vesght Miaeral capbon H-Unit $q$-Unit Meight

\begin{tabular}{|c|c|c|c|c|c|c|c|c|c|}
\hline \multirow[t]{7}{*}{ Soin } & 6.5 & 2.5 & 97.5 & 116.25 & 4533.75 & 16.5 & 116.25 & 9333.7 & 07850 \\
\hline & 19.6 & 12.5 & 97.5 & 245 & 1715 & 66.1 & 361.25 & 6240.75 & 9.1829186 .020 \\
\hline & .36 & 30 & 70 & 250.8 & 385.2 & 74.16 & 612.05 & 6033.15 & 2.0667076 .315 \\
\hline & S1 & 30 & 30 & 329 & 329 & 01.04 & P41.05 & 7162.15 & 6.4969763 .584 \\
\hline & 26 & 70 & 30 & 939.6 & 188.1 & 87.32 & 1380.65 & 7351.35 & 9.0370946 .372 \\
\hline & .16 & 87.5 & 12.5 & 346.5 & 19.9 & 91.28 & 1727.15 & 7400.85 & 9.7039533 .16436 \\
\hline & 8.79 & 97.5 & 2.5 & 857.023 & 21.975 & 100.07 & 2584.175 & 7422.823 & 100 \\
\hline \multirow[t]{7}{*}{ ois } & .7 & 2.5 & 17.5 & 146.75 & 5723.25 & 31.7 & 146.75 & \multicolumn{2}{|c|}{5723.2572 .1314392 .86764} \\
\hline & 13.1 & 12.5 & 87.3 & 163.75 & 1146.25 & 71.8 & 310.5 & \multicolumn{2}{|c|}{6869.586 .5778184 .90905} \\
\hline & 7.87 & 30 & 70 & 230.1 & 536.9 & 79.17 & 540.6 & \multicolumn{2}{|c|}{7406.493 .3445573 .72371} \\
\hline & 6.78 & so & 50 & 339 & 339 & 86.25 & 879.6 & \multicolumn{2}{|c|}{7743.497 .6170457 .24961} \\
\hline & 4.36 & 70 & 30 & 305.2 & 130.8 & 90.61 & 1184.8 & \multicolumn{2}{|c|}{7876.291 .2655542 .11629} \\
\hline & 3.5 & 87.5 & 12.5 & 306.25 & 43.75 & 94.11 & 1491.05 & \multicolumn{2}{|c|}{7919.9599 .0169427 .53186} \\
\hline & 3.81 & 97.5 & 2.5 & 966.475 & 14.525 & 99.12 & 2057.525 & 7934.473 & 100 \\
\hline \multirow[t]{7}{*}{ Fee } & 33.2 & 2.5 & 97.5 & 133 & 3187 & 93.2 & 133 & \multicolumn{2}{|c|}{510762.9467492 .44619} \\
\hline & 23.3 & 12.5 & 87.5 & 291.25 & 2038.75 & 76.5 & 421.25 & \multicolumn{2}{|c|}{7225.7587 .6879579 .90447} \\
\hline & 8.88 & 30 & 70 & 266.1 & 621.6 & 85.38 & 690.65 & \multicolumn{2}{|c|}{7897.3595 .2315660 .77412} \\
\hline & 4.17 & 30 & 30 & 223.5 & 223.5 & 89.15 & 914.15 & \multirow{2}{*}{\multicolumn{2}{|c|}{$8184.2599 .319813 j .05220$}} \\
\hline & 3.78 & 70 & jo & 264.6 & 113.4 & 93.63 & 1878.75 & & \\
\hline & 4.01 & 87.5 & 12.5 & 350.875 & 50.125 & 97.64 & 1529.625 & \multicolumn{2}{|c|}{8234.37599 .9281013 .12404} \\
\hline & 2.37 & 97.5 & 2.5 & 231.073 & 3.925 & 100.01 & 1760.7 & 8240.3 & 100 \\
\hline
\end{tabular}


SANPLE: Pit/30 ein

ASSAY: ASH

\begin{tabular}{|c|c|c|c|c|c|c|c|c|c|c|}
\hline S6 & & Dolvian & & cune & LATIVE & FLOAT & cunt & LATIYE & sinx & \\
\hline SII FLI & עT & assay & PROS & עT & P2OS & ASSAi & U1 & PROS & ISSAY & 2 \\
\hline 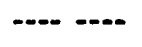 & -.-. & $\cdots$ & $\ldots$ & $\cdots$ & $\cdots$ & $\cdots$ & -..- & 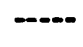 & $\cdots$ & $\cdots$ \\
\hline 1.20 & 18.31 & 4.39 & 0.84 & 18.31 & 0.84 & 1.59 & 100.00 & 13.34 & 13.34 & 9.15 \\
\hline 1.201 .30 & 12.54 & 4.12 & 1.75 & 60.85 & 2.59 & 4.26 & 81.69 & 12.50 & 15.30 & 39.58 \\
\hline 1.301 .40 & 27.24 & 8.83 & 2.11 & 88.09 & 3.00 & 5.67 & 39.15 & 10.74 & 27.44 & 74.17 \\
\hline 1.401 .70 & 3.83 & 50.44 & 1.98 & 92.02 & 6.98 & 7.59 & 11.91 & 8.34 & 70.01 & 90.05 \\
\hline 1.70 & 7.98 & 79.65 & 6.36 & 100.00 & 13.34 & 13.34 & 7.98 & 6.36 & 79.65 & 96.01 \\
\hline
\end{tabular}

SAAPLE: Pit/30 DiA

ASSAY: SULFUR

\begin{tabular}{|c|c|c|c|c|c|c|c|c|c|c|}
\hline S6 & & IMoivi] & & Conit & AIIVE & LonT & & IIVE & SIII & \\
\hline SHI FLT & VT & ASSAY & PROD & vi & PROS & ASSAY & VT & PROI & ASSAY & \\
\hline & ..... & & & 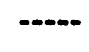 & 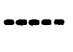 & -- & 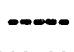 & 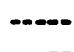 & & \\
\hline 1.20 & 18.31 & 3.73 & 0.68 & 18.31 & 0.68 & 3.73 & 100.00 & 4.73 & 4.73 & \\
\hline $\begin{array}{ll}1.20 & 1.30\end{array}$ & 12.54 & 3.36 & 1.43 & 60.85 & 2.11 & 3.17 & 81.69 & 9.04 & 4.95 & 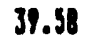 \\
\hline 1.301 .40 & 27.21 & 4.36 & 1.19 & 38.09 & 3.30 & 3.73 & 39.15 & 2.61 & 6.67 & 74.47 \\
\hline 1.101 .70 & 3.93 & 8.05 & 0.32 & 12.02 & 3.12 & 3.93 & 11.11 & 1.13 & 11.17 & iv \\
\hline 1.70 & 7.98 & 13.90 & 1.11 & 100.00 & 4.73 & 4.73 & 7.11 & 1.11 & 13.10 & 96. \\
\hline
\end{tabular}

:AMPLE: Pit/3O ain

ASSAY: PYRIIIC

\begin{tabular}{|c|c|c|c|c|c|c|c|c|c|c|}
\hline S6 & & DIVII & & $\operatorname{con} 1$ & AlIVE & FLOAT & & DIIVE & SINK & \\
\hline SIII FLT & UT & Assar & PROB & ע & PROS & AssaY & $n$ & PRDD & Assal & $l$ \\
\hline$\cdots$ & 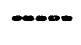 & & 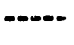 & 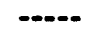 & -.-- & 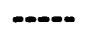 & 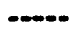 & -.-- & 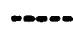 & \\
\hline 1.20 & 18.31 & 0.18 & 0.09 & 18.31 & 0.01 & 0.18 & 100.01 & 1.61 & 1.61 & 9.1 \\
\hline$\therefore .201 .30$ & 12.54 & 0.54 & 0.23 & 60.85 & 0.32 & 0.52 & 81.68 & 1.52 & 1.86 & 39.9 \\
\hline 1.301 .40 & 27.24 & 1.83 & 0.50 & 88.09 & 0.12 & 0.13 & 37.15 & 1.29 & 3.30 & 74.17 \\
\hline ¿.41 1.70 & 3.93 & 9.69 & 0.22 & 92.02 & 1.05 & 1.14 & 11.11 & 0.79 & 6.11 & 90.05 \\
\hline 1.70 & 7.98 & 7.06 & 0.56 & 100.08 & 1.61 & 1.61 & 7.90 & 0.36 & 7.16 & 96.01 \\
\hline
\end{tabular}


SAHPLE: Pit/10 oin

ASSAY: ASH

\begin{tabular}{|c|c|c|c|c|c|c|c|c|c|c|}
\hline 56 & & DIVIDU & & cone & LAIIVE & FLOAT & CUn & LATIVE & SINK & \\
\hline SUI FLI & UI & ASSAY & PROS & ז & Pros & ASSAY & עT & PROS & ASSAY & $I$ \\
\hline$\cdots$ & --.-- & -...- & $\cdots$ & -.... & -..-- & ..... & -..-. & $\cdots$ & $\cdots$ & $=$ \\
\hline 1.20 & 31.73 & 3.96 & 1.26 & 31.73 & 1.26 & 3.96 & 100.00 & 12.16 & 12.16 & 13.86 \\
\hline 1.201 .30 & 50.62 & 3.59 & 1.82 & 82.35 & 3.07 & 3.73 & 68.27 & 10.90 & 15.17 & 57.04 \\
\hline 1.301 .40 & 5.26 & 16.33 & 0.86 & 87.61 & 3.93 & 1.49 & 17.65 & 9.09 & 51.48 & 84.98 \\
\hline 1.101 .70 & 3.31 & 37.29 & 1.23 & 90.92 & 3.17 & 3.68 & 12.39 & 8.23 & 66.11 & 89.26 \\
\hline 1.70 & 9.08 & 77.02 & 6.99 & 100.00 & 12.16 & 12.16 & 9.00 & 6.99 & 77.02 & 95.16 \\
\hline
\end{tabular}

SAMPLE: Pit/10 a In

ASSAY: SULFUR

\begin{tabular}{|c|c|c|c|c|c|c|c|c|c|c|}
\hline SG & & MaIvid & & cunl & LAIIVE & fLOAT & & AIIVE & SIMI & \\
\hline SML FLI & ו & ASSAY & PROS & UI & PRBD & ASSAY & זו & PROI & ASSAY & 2 \\
\hline$\cdots$ & $\cdots$ & $\cdots$ & $\cdots$ & $\cdots$ & 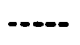 & 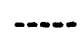 & $\cdots$ & - & $\cdots$ & \\
\hline 1.20 & 31.73 & 3.30 & 1.05 & 31.73 & 1.05 & 3.30 & 100.00 & 1.49 & 4.19 & 9.8 \\
\hline 1.201 .30 & 30.62 & 3.29 & 1.65 & 82.35 & 2.69 & 3.27 & 68.27 & 3.45 & 9.05 & 57.0 \\
\hline .301 .40 & 9.26 & 4.86 & 0.26 & 87.61 & 2.95 & 3.36 & 17.65 & 1.80 & 10.20 & 97. \\
\hline 1.401 .70 & 3.31 & 7.16 & 0.24 & 90.92 & 3.18 & 3.50 & 12.39 & 1.54 & 12.17 & 89.26 \\
\hline 1.70 & 9.08 & 14.40 & 1.31 & 100.00 & 1.19 & 4.49 & 9.08 & 1.31 & 19.40 & 0.46 \\
\hline
\end{tabular}

SAAPLE: Pit/10 a

ASSAY: PrruIII

\begin{tabular}{|c|c|c|c|c|c|c|c|c|c|c|}
\hline 56 & & NDIVIDU & & chisu & AfIVE & flont & conery & AIIIVE & SIM & \\
\hline ShM FLI & II & ASSAY & PROD & ע & Pres & ASSAY & זע & PROD & ASSAY & $l$ \\
\hline - -... & $\cdots$ & $\cdots$ & -...- & $\cdots$ & -...- & 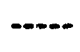 & $\cdots$ & 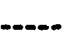 & -...." & \\
\hline 1.20 & 31.73 & 0.14 & 0.11 & 31.73 & 0.14 & 0.44 & 100.00 & 1.12 & 1.12 & 3.8 \\
\hline 1.201 .30 & 50.62 & 0.48 & 0.24 & 82.35 & 0.38 & 0.16 & 01.27 & 1.28 & 1.10 & .04 \\
\hline 1.301 .40 & 5.26 & 2.14 & 0.13 & 07.61 & 0.51 & 0.58 & 17.65 & 1.04 & 3.10 & 0.18 \\
\hline 1.101 .70 & 3.31 & 5.19 & 0.17 & 90.92 & 0.18 & 0.75 & 12.31 & 0.91 & 7.37 & 87.26 \\
\hline$\therefore .70$ & 9.08 & 8.17 & 0.74 & 100.00 & 1.12 & 1.42 & 9.08 & 0.74 & 8.17 & 0.46 \\
\hline
\end{tabular}


SAMPLE: Pil/S ain

ASSAY: ASH

\begin{tabular}{|c|c|c|c|c|c|c|c|c|c|c|}
\hline S6 & & MIVIDI & & canl & ILATIVE & FLOAT & CUne & ILAYIVE & SIMK & \\
\hline SHI FLI & II & ASSAY & PROD & II & PROI & ASSAY & " & PROD & ASSAY & l \\
\hline ....... & $\cdots$ & $\cdots$ & 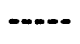 & $\cdots$ & $\cdots$ & $\cdots$ & $\cdots$ & $\cdots$ & $\cdots$ & $\cdots$ \\
\hline 1.20 & 37.15 & 3.19 & 1.30 & 37.15 & 1.30 & 3.19 & 100.00 & 13.26 & 13.26 & 18.58 \\
\hline 1.201 .30 & 43.78 & 3.47 & 1.52 & 80.93 & 2.82 & 3.48 & 62.85 & 11.96 & 19.03 & 59.04 \\
\hline 1.301 .40 & 9.14 & 13.63 & 0.70 & 86.07 & 3.52 & 4.09 & 19.07 & 10.44 & 34.73 & 83.50 \\
\hline 1.101 .70 & 3.89 & 54.96 & 2.11 & 89.96 & 5.65 & 6.29 & 13.93 & 9.74 & 69.92 & 88.01 \\
\hline 1.70 & 10.04 & 73.72 & 7.60 & 100.00 & 13.26 & 13.26 & 10.04 & 7.60 & 75.72 & 94.98 \\
\hline
\end{tabular}

SAIPLE: Pit/S ain

ASSAY: SULFUR

\begin{tabular}{|c|c|c|c|c|c|c|c|c|c|c|}
\hline 56 & & NoIVIDI & & cun & LATIYE & FLOAT & cund & LATIYE & SILK & \\
\hline SMI FLI & $H T$ & ASSAY & PROI & II & PRQS & AssaY & ז & PROS & assar & 2 \\
\hline -...- -... & $\cdots$ & $\cdots$ & -..- & $\cdots$ & $\cdots$ & $\cdots$ & $\cdots$ & - & $\cdots$ & . \\
\hline 1.20 & 37.15 & 3.29 & 1.22 & 37.15 & 1.22 & 3.29 & 100.00 & 4.57 & 1.57 & 18.38 \\
\hline 1.201 .30 & 13.78 & 3.13 & 1.37 & 80.13 & 2.59 & 3.20 & 62.85 & 3.35 & 9.33 & 59.04 \\
\hline 1.301 .10 & 9.14 & 4.06 & 0.21 & 86.07 & 2.80 & 3.23 & 19.07 & 1.98 & 10.37 & 83.50 \\
\hline 1.101 .70 & 3.89 & 7.29 & 0.28 & 89.96 & 3.08 & 3.43 & 13.13 & 1.77 & 12.69 & 88.01 \\
\hline 1.70 & 10.04 & 14.80 & 1.19 & 100.00 & 4.97 & 1.37 & 10.04 & 1.49 & 11.80 & 94.98 \\
\hline
\end{tabular}

SAMPLE: Pit/S aIN

ASSAY: PYRITIC

\begin{tabular}{|c|c|c|c|c|c|c|c|c|c|c|}
\hline \multirow{3}{*}{$\begin{array}{c}\text { S6 } \\
\text { SUl } \\
\end{array}$} & \multicolumn{3}{|c|}{ INUIVIDUAL } & \multicolumn{3}{|c|}{ CUHULATIVE FloAt } & \multicolumn{3}{|c|}{ CUMU ATIVE SIUI } & \multirow[b]{2}{*}{2} \\
\hline & I & ASSAY & Prog & ע & PROI & ASSAY & $\boldsymbol{n}$ & PAOS & Assir & \\
\hline & . & $\cdots$ & - & 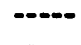 & 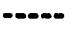 & . & 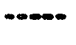 & -..- & 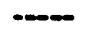 & 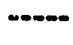 \\
\hline 1.20 & 37.15 & 0.24 & 0.01 & 37. & 0.09 & 0.24 & 100.00 & 1.19 & 1.41 & 18.38 \\
\hline 201.30 & 13.78 & 0.28 & 0.13 & 80.13 & 0.22 & 0.27 & 12.85 & 1.10 & 2.23 & 59.01 \\
\hline 1.301 .40 & 3.11 & 1.92 & 0.10 & 26.07 & 0.31 & 0.37 & 19.07 & 1.27 & 6.67 & 83.31 \\
\hline 1.401 .70 & 3.89 & 3.64 & 0.22 & 89.16 & 0.33 & 0.59 & 13.13 & 1.17 & 8.13 & 88.01 \\
\hline 1.70 & 10.04 & 9.51 & 0.95 & 100.00 & 1.49 & 1.49 & 10.04 & 0.95 & 9.51 & 94.98 \\
\hline
\end{tabular}


SAMPLE: EIK/JO Din

ASSAY: ASH

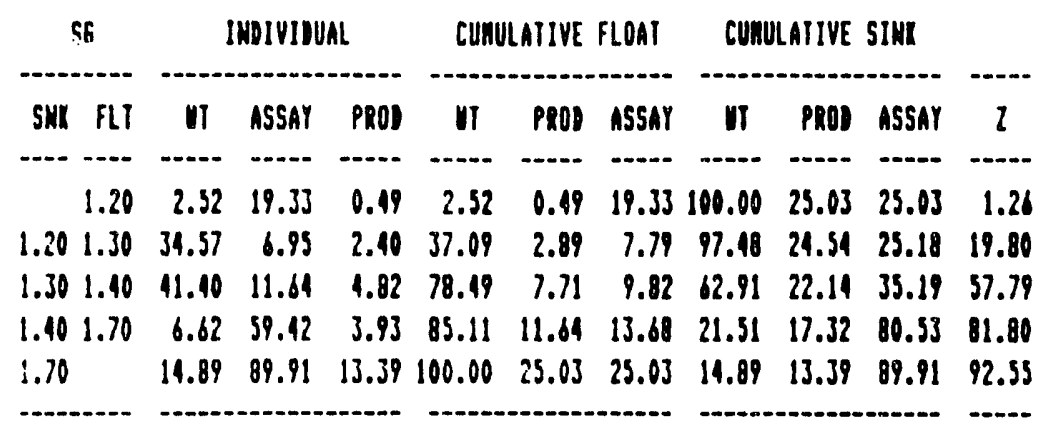

SARPLE: Elk/JO ain

ASSAY: SULFUR

\begin{tabular}{|c|c|c|c|c|c|c|c|c|c|c|}
\hline 56 & & NaIVIDL & & cuns & ATIVE & FLOAT & & AII & sint & \\
\hline SHII FLI & עT & ASSAY & PROS & VI & PROS & ASSAY & ע & PROS & ASSAY & 2 \\
\hline 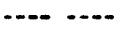 & $\cdots$ & -...- & $\cdots$ & $\cdots$ & $\cdots$ & 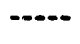 & -...- & $\cdots$ & 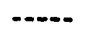 & 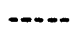 \\
\hline 1.20 & 2.52 & 1.23 & 0.03 & 2.52 & 0.03 & 1.23 & 100.00 & 1.81 & 1.81 & 1.2 \\
\hline 1.201 .30 & 34.57 & 1.16 & 0.10 & 37.09 & 0.13 & 1.16 & 97.48 & 1.57 & 1.62 & 19.80 \\
\hline 1.301 .10 & 91.40 & 1.16 & 0.48 & 78.19 & 0.91 & 1.16 & 62.91 & 1.17 & 1.87 & 57.79 \\
\hline$\therefore .401 .70$ & 6.62 & 2.06 & 0.14 & 85.11 & 1.05 & 1.23 & 21.51 & 0.69 & 3.22 & 81.80 \\
\hline$\therefore .70$ & 14.89 & 3.74 & 0.56 & 100.00 & 1.61 & 1.61 & 14.89 & 0.56 & 3.74 & 92.55 \\
\hline
\end{tabular}

DNAPLE: E! $k / 30$ oin

issar: PYRIIIC

\begin{tabular}{|c|c|c|c|c|c|c|c|c|c|c|}
\hline 56 & & DIVIDU & & & AIIVE & FLOAT & & CAIIVE & SIM & \\
\hline SNI FLT & ע & ASSAY & PROS & VT & PROI & AsSAY & U & PROO & ASSAY & l \\
\hline$\cdots$ & $\ldots$ & -... & $\cdots$ & 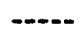 & $\cdots$ & -..-. & 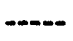 & -..- & -...- & 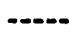 \\
\hline 1.20 & 2.52 & 0.68 & 0.02 & 2.52 & 0.02 & 0.68 & 100.00 & 1.02 & 1.02 & 1.2 \\
\hline 1.201 .30 & 34.57 & 0.63 & 0.22 & 37.09 & 0.23 & 0.63 & 97.48 & 1.00 & 1.02 & 19.80 \\
\hline :.30 1.10 & 11.40 & 0.48 & 0.20 & 78.49 & 0.43 & 0.55 & 62.91 & 0.78 & 1.29 & 57.79 \\
\hline 1.101 .70 & 6.82 & 1.55 & 0.10 & 85.11 & 0.54 & 0.63 & 21.51 & 0.58 & 2.71 & 01.80 \\
\hline 1.70 & 14.89 & 3.22 & 0.18 & 100.00 & 1.02 & 1.02 & 14.89 & 0.48 & 3.22 & 92.55 \\
\hline
\end{tabular}


SAHPLE: EIK/10 DiR

ASSAY: ASH

\begin{tabular}{|c|c|c|c|c|c|c|c|c|c|c|}
\hline 56 & & DDIV & & Whe & CATIVE & OAT & DU & LAT. & SIMR & \\
\hline SHI FLI & זע & ASSAY & PROS & UT & PROI & ASSAY & VI & PrOS & ASSAY & $l$ \\
\hline 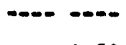 & -..- & 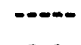 & $\cdots$ & $\cdots$ & --- & $\cdots$ & -..- & $\cdots$ & $\cdots$ & \\
\hline 1.20 & 26.21 & 2.96 & 0.75 & 26.21 & 0.75 & 2.16 & 100.00 & 23.19 & 23.19 & 10 \\
\hline . 201.30 & 12.90 & 4.60 & 1.97 & 69.11 & 2.72 & 3.94 & 73.79 & 22.41 & 30.41 & 17.80 \\
\hline .30 1.40 & 9.93 & 18.00 & 1.07 & 75.04 & 3.79 & 5.05 & 30.89 & 20.46 & 66.23 & 72.07 \\
\hline 1.101 .70 & 7.01 & 50.55 & 3.54 & 82.05 & 7.33 & 8.94 & 29.96 & 19.40 & 77.71 & 78.54 \\
\hline 1.70 & 17.95 & 88.32 & 19.85 & 100.00 & 23.19 & 23.19 & 17.95 & 15.85 & 88.32 & 91.03 \\
\hline
\end{tabular}

SAAPLE: EJK/10 Ain

ASSAY: SULFUR

\begin{tabular}{|c|c|c|c|c|c|c|c|c|c|c|}
\hline 56 & & MaIvis & & Cuniu & AIIVE & OAT & & AII & simk & \\
\hline SHI FLT & ו & ASSAY & PROS & ขT & PROS & ASSAY & II & PROS & ASSAY & 2 \\
\hline$\cdots$ & 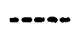 & -..- & --..- & -- & $\cdots$ & 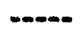 & $\cdots$ & $\cdots$ & $\cdots$ & $-\infty$ \\
\hline 1.20 & 26.21 & 0.72 & 0.19 & 26.21 & 0.19 & 0.72 & 100.00 & 1.45 & 1.19 & 1.16 \\
\hline 201.30 & 12.90 & 0.96 & 0.41 & 69.11 & 0.60 & 0.87 & 73.79 & 1.27 & 1.71 & 47. \\
\hline 1.301 .40 & 9.93 & 1.30 & 0.08 & 79.04 & 0.68 & 0.10 & 30.89 & 0.85 & 2.76 & 72.07 \\
\hline .101 .70 & 7.01 & 1.52 & 0.11 & 82.05 & 0.78 & 0.16 & 24.96 & 0.78 & 3.11 & 78.51 \\
\hline 1.70 & 17.99 & 3.73 & 0.67 & 100.00 & 1.13 & 1.15 & 17.95 & 0.67 & 3.73 & 91.03 \\
\hline
\end{tabular}

SAHPLE: EIK/10 OIQ

ASSAY: PYRIIIC

\begin{tabular}{|c|c|c|c|c|c|c|c|c|c|c|}
\hline 56 & & Marivis & & & AIIVE & Flont & cont & AIIVE & stan & \\
\hline SNAK FLT & זע & ASSAY & PROS & ט & Pros & ASSAT & $\| T$ & PROI & ASSAY & $l$ \\
\hline -... & $\cdots$ & ---. & $\cdots$ & $\cdots$ & $\cdots$ & $\cdots$ & $\cdots$ & $\cdots$ & 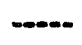 & \\
\hline 1.20 & 26.21 & 0.18 & 0.05 & 26.21 & 0.05 & 0.18 & 100.00 & 0.85 & 0.15 & 13. \\
\hline 201.30 & 92.90 & 0.18 & 0.01 & 69.11 & 0.12 & 0.11 & 73.77 & 0.81 & 1.01 & 17.66 \\
\hline .301 .40 & 9.93 & 0.62 & 0.01 & 75.04 & 0.16 & 0.21 & 50.89 & 0.73 & 2.36 & 72.07 \\
\hline .101 .70 & 7.01 & 1.07 & 0.08 & 82.05 & 0.24 & 0.29 & 24.16 & 0.69 & 2.77 & 71.54 \\
\hline 1.78 & 17.95 & 3.11 & 0.62 & 100.00 & 0.85 & 0.05 & 17.15 & 0.62 & 3.11 & 91.03 \\
\hline
\end{tabular}


APPENDIX B: Contour Diagrams

B-1 


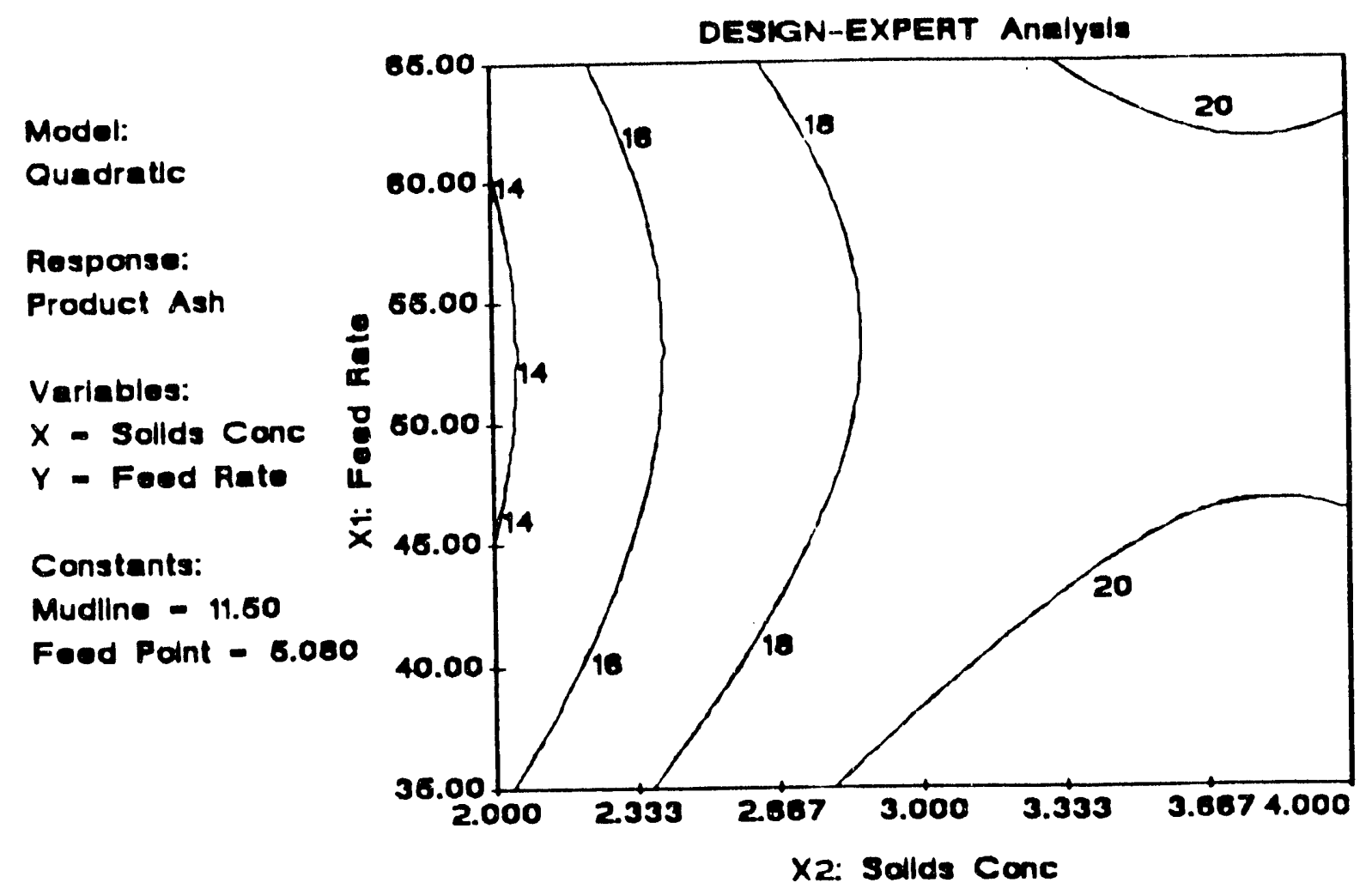

Figure B1: Product Ash Content as a function of Feed Rate and Feed Solids Concentration.

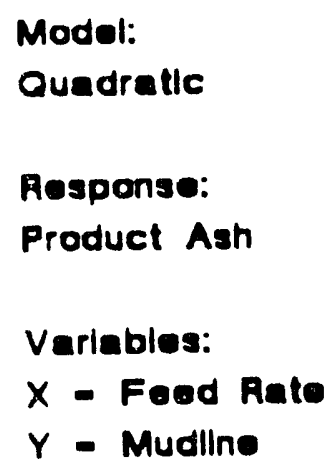

Constants:

Sollds Conc -3.000 Fead Point - 6.000

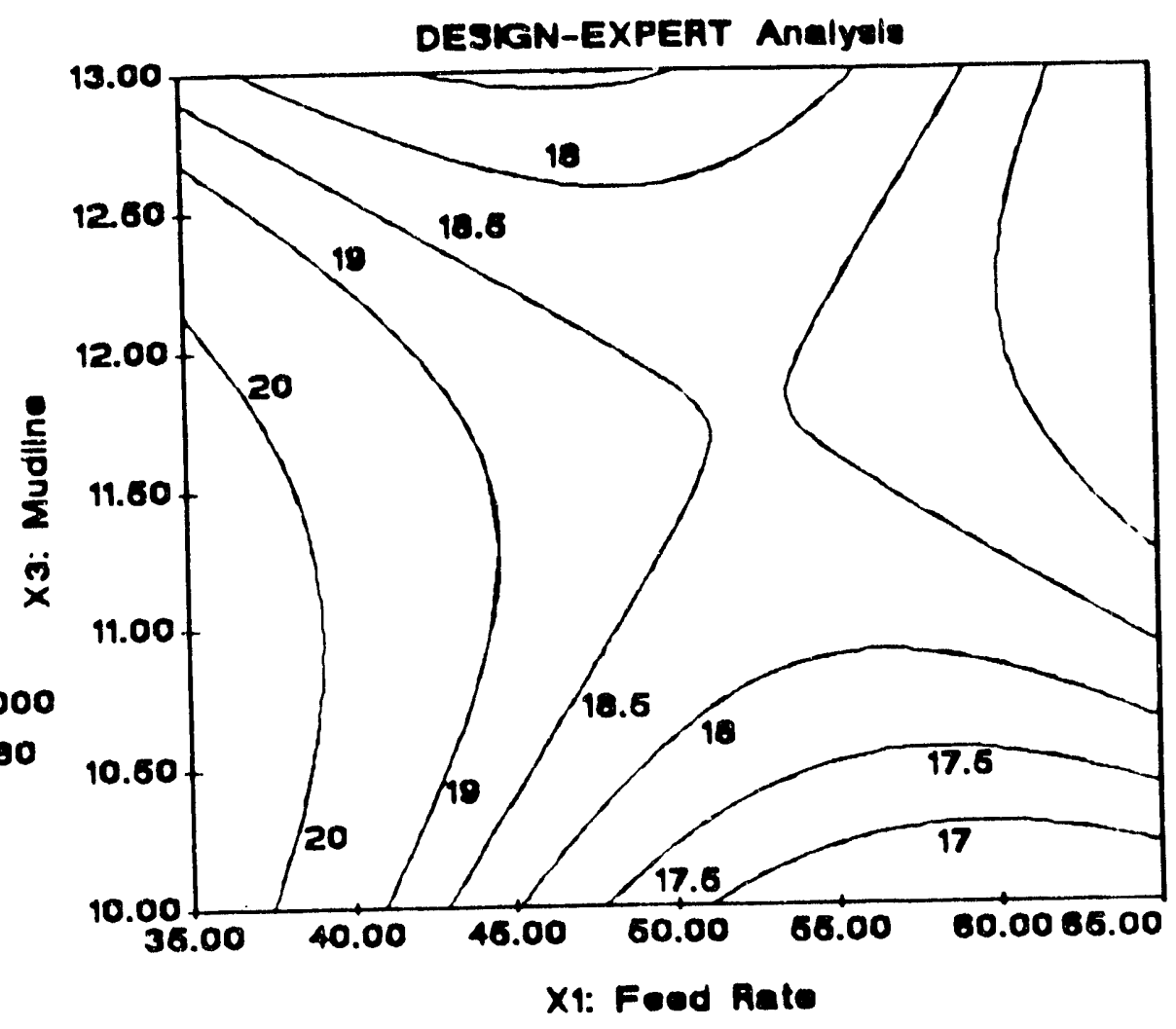

Figure B2: Product Ash Content as a function of Feed Rate and Mudline Depth. 
Modal:

Quadratic

Response:

Product Aah

Varlables:

$X$ - Foed Rato

$Y$ - Food Point

Constants:

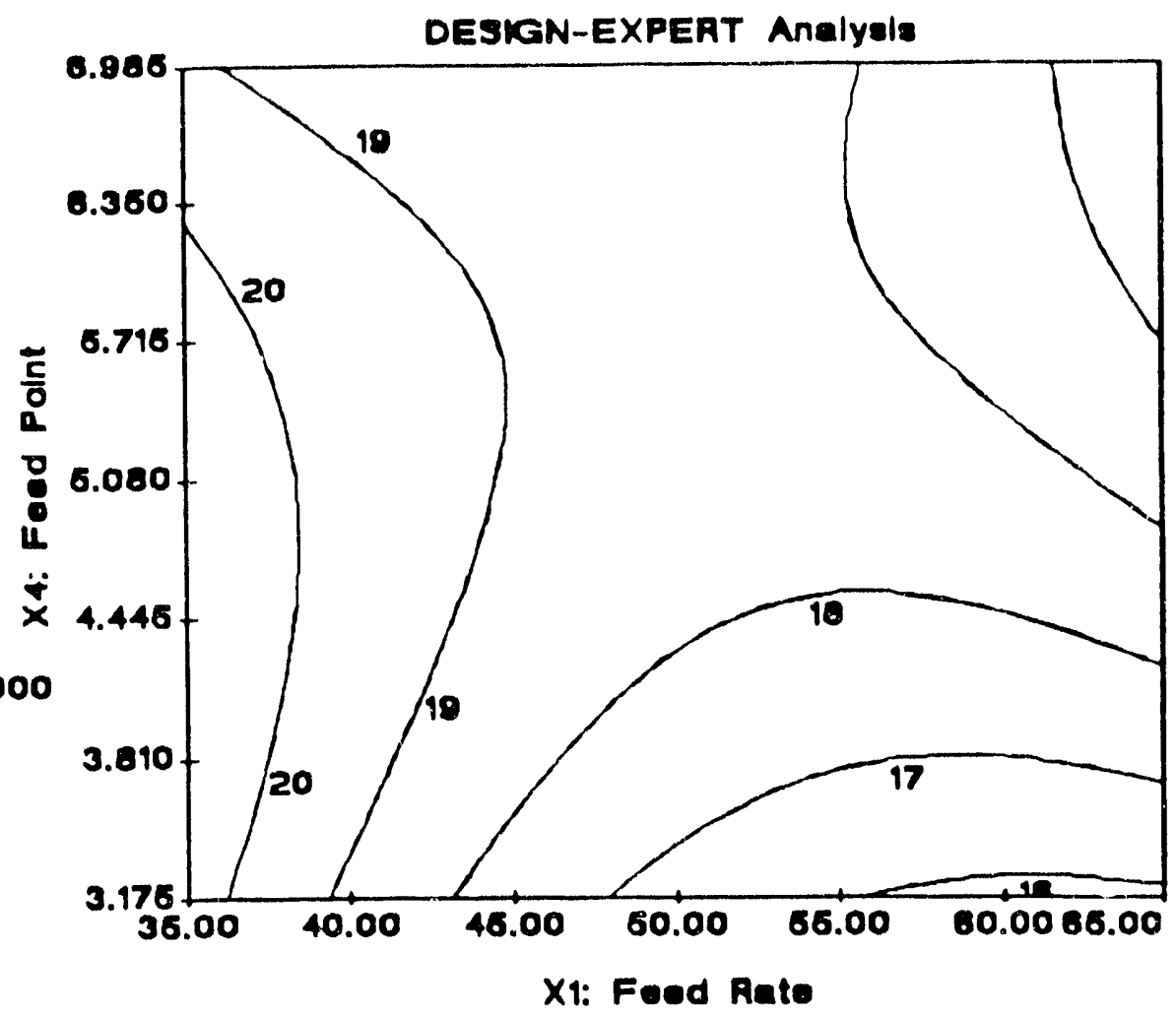

Figure B3: Product Ash Content as a function of Feed Rate and Feed Inlet Point.

Model:

Quadratle

Response:

Product Ash

Variables:

$X$ - Sollds Cone

$Y$ - Mudline

Constants:

Foed Rate $=60.00$

Foed Point $=6.080$

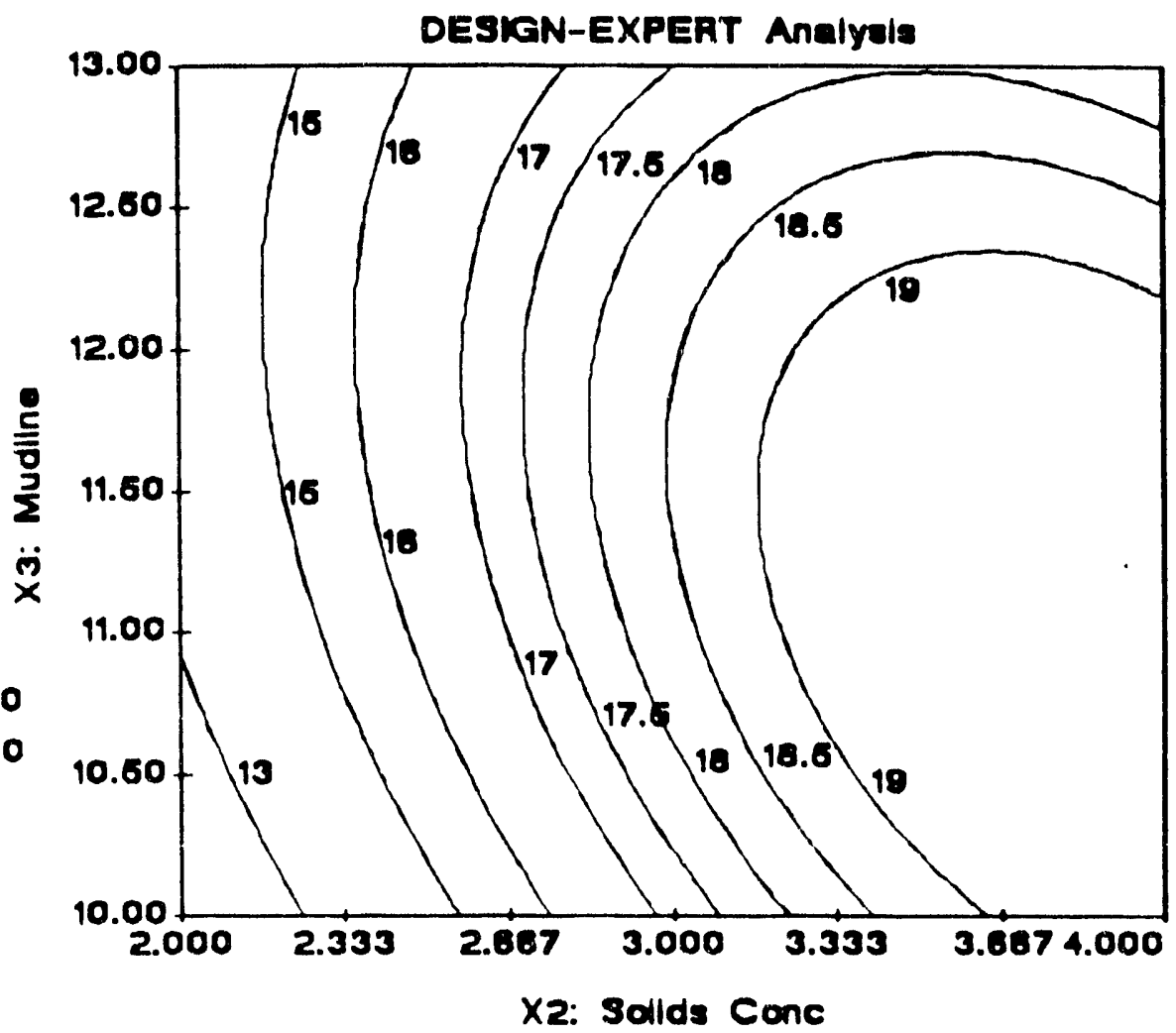

Figure B4: Product Ash Content as a function of Feed Solids Concentration and Mudline Depth. 


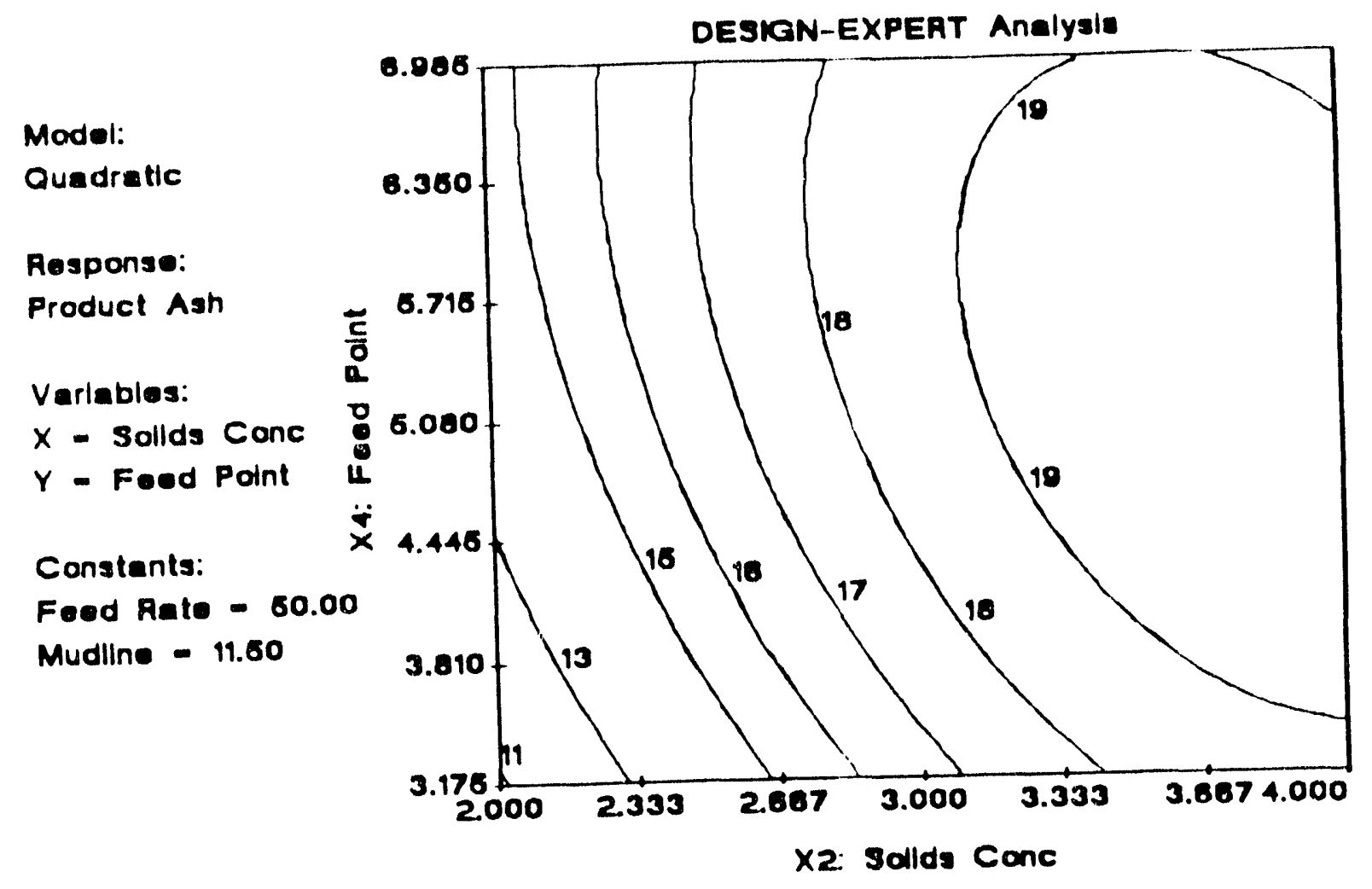

Figure B5: Product Ash Content as a function of Feed Solids Concentration and Feed Inlet Point.

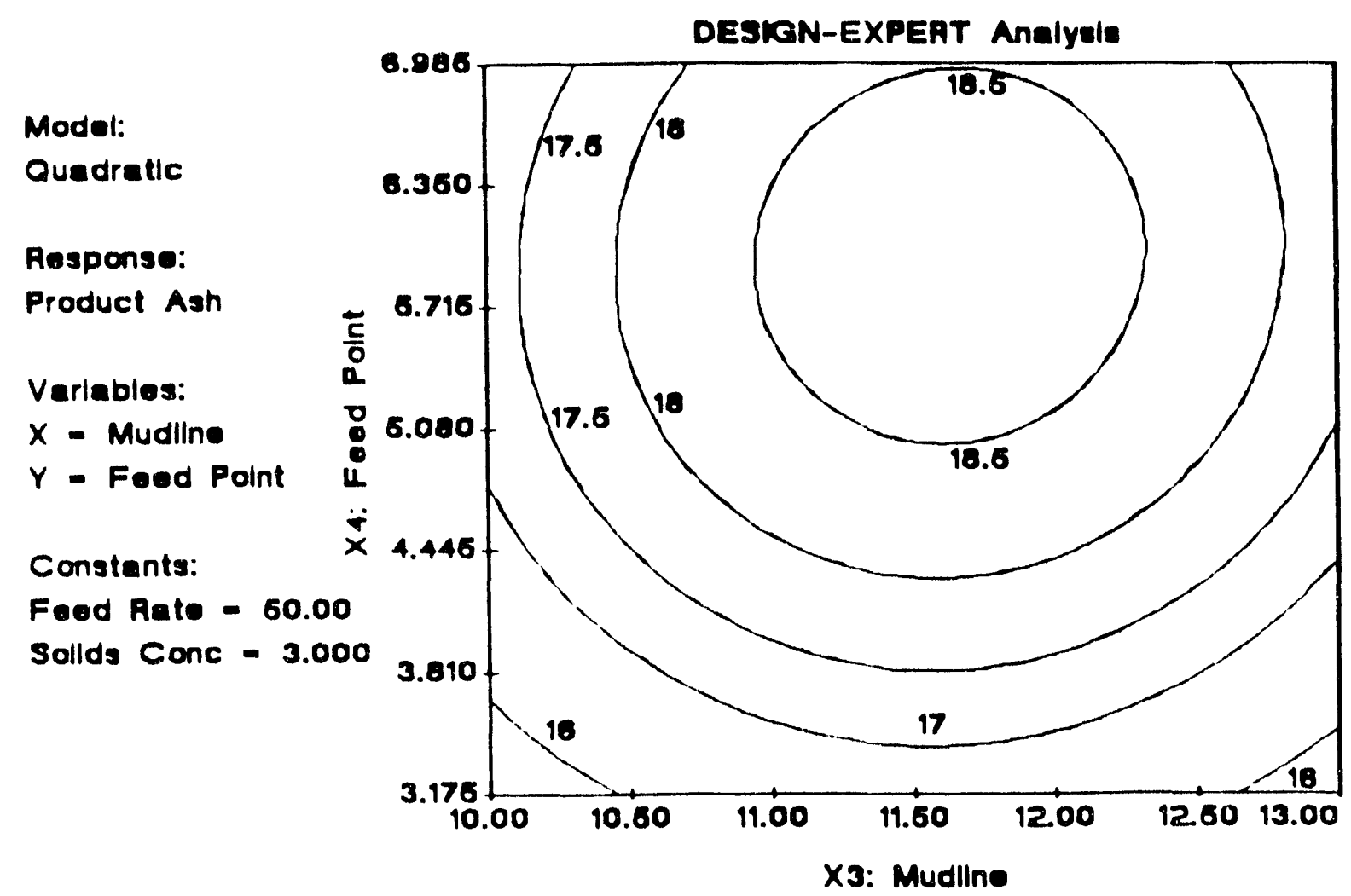

Figure B6: Product Ash Content as a function of Mudline Depth and Feed Inlet Point. 


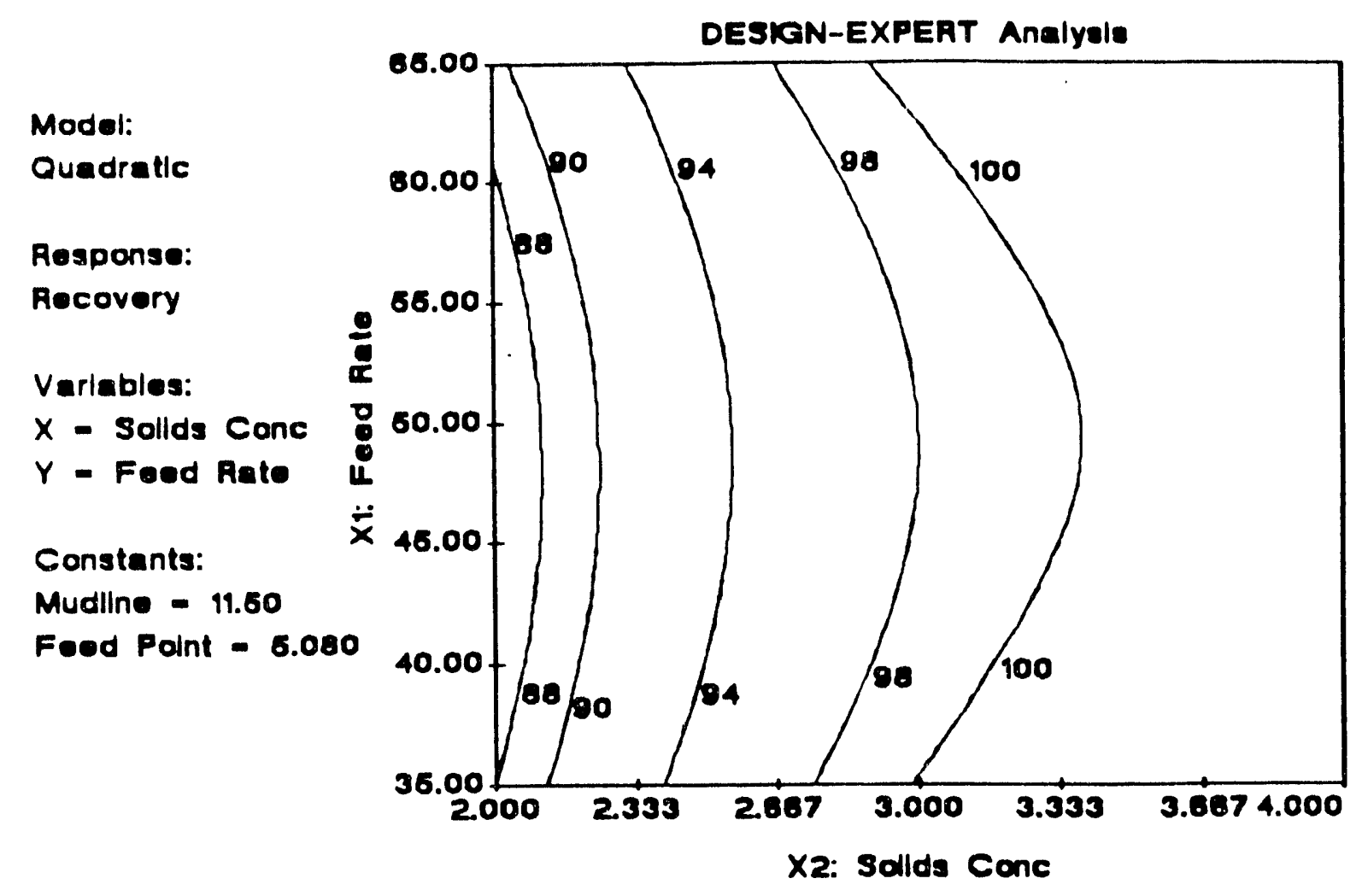

Figure B7: Combustible Recovery as a function of Feed Rate and Feed Solids Concentration.

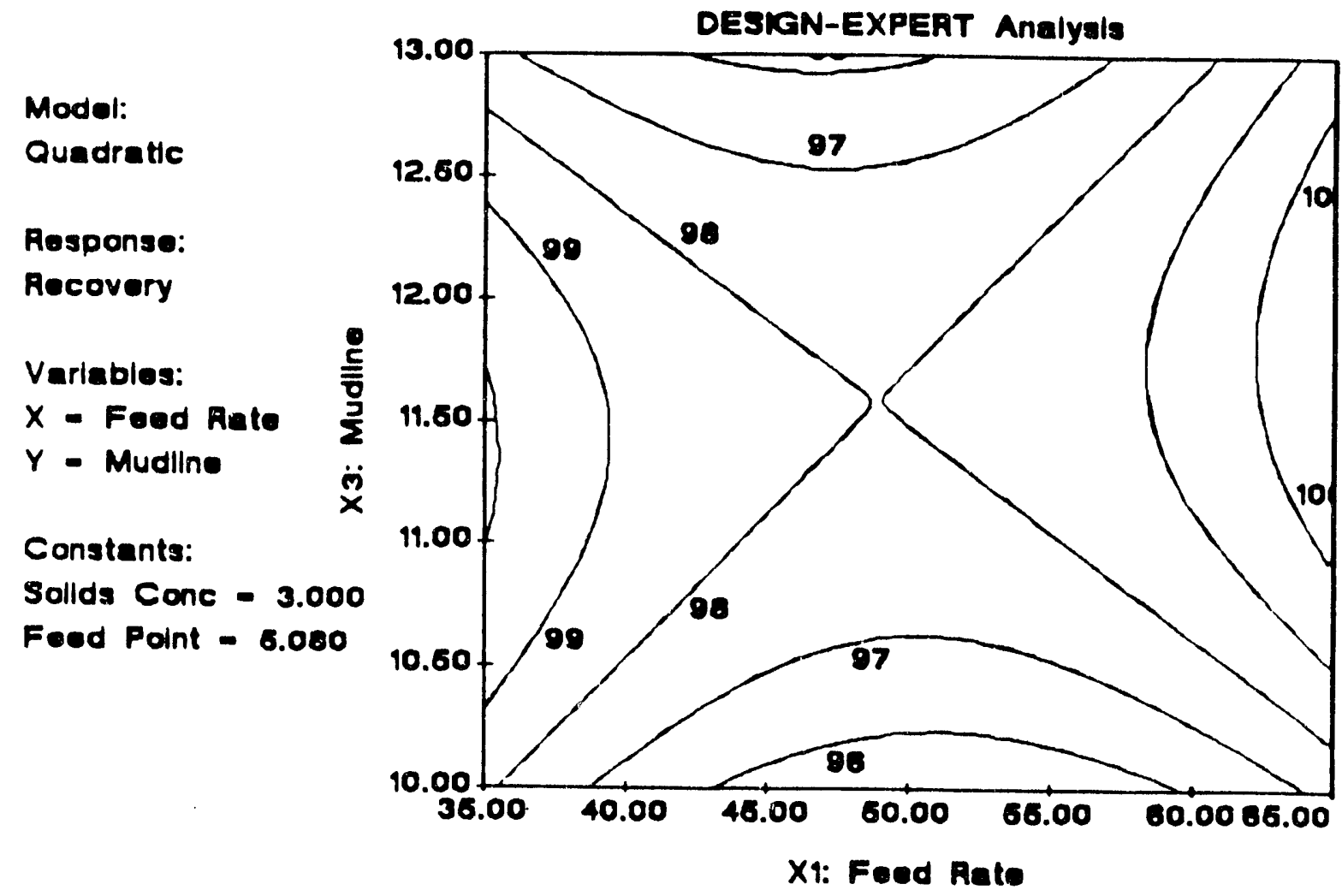

Figure B8: Combustible Recovery as a function of Feed Rate and Mudline Depth. 
Model:

Quadratlc

Response:

Recovery

Variables:

$X$ - Foed Rate

$Y$ - Foed Paint

Constents:

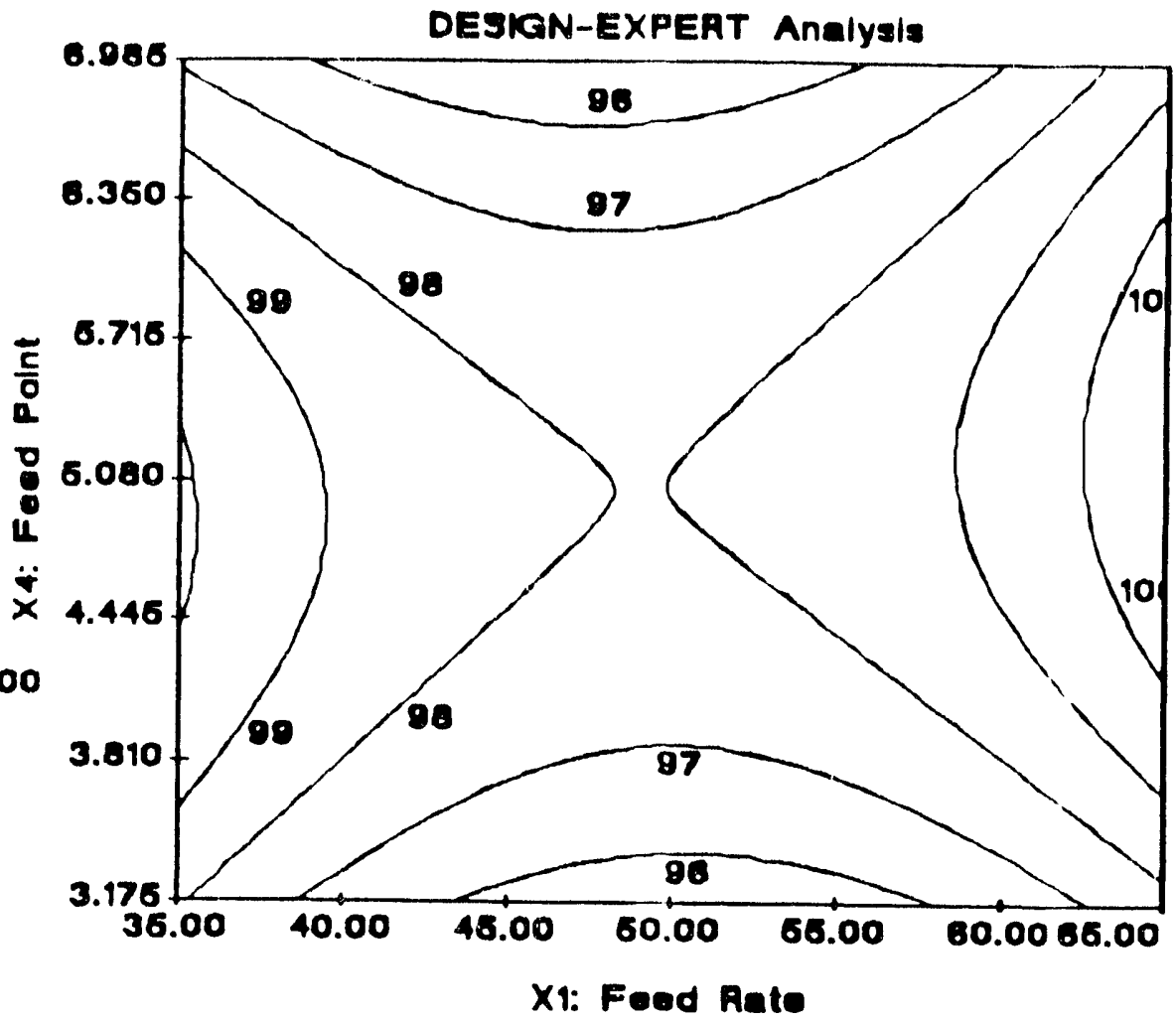

Figure B9: Combustible Recovery as a function of Feed Rate and Feed Inlet Point.

Model:

Quedratlc

Rిesponse:

Aecovery

Variables:

$X$ - Sollds Cone

$Y$ - Mudline

Constants:

Foed Rate - 60.00

Feod Point -6.000

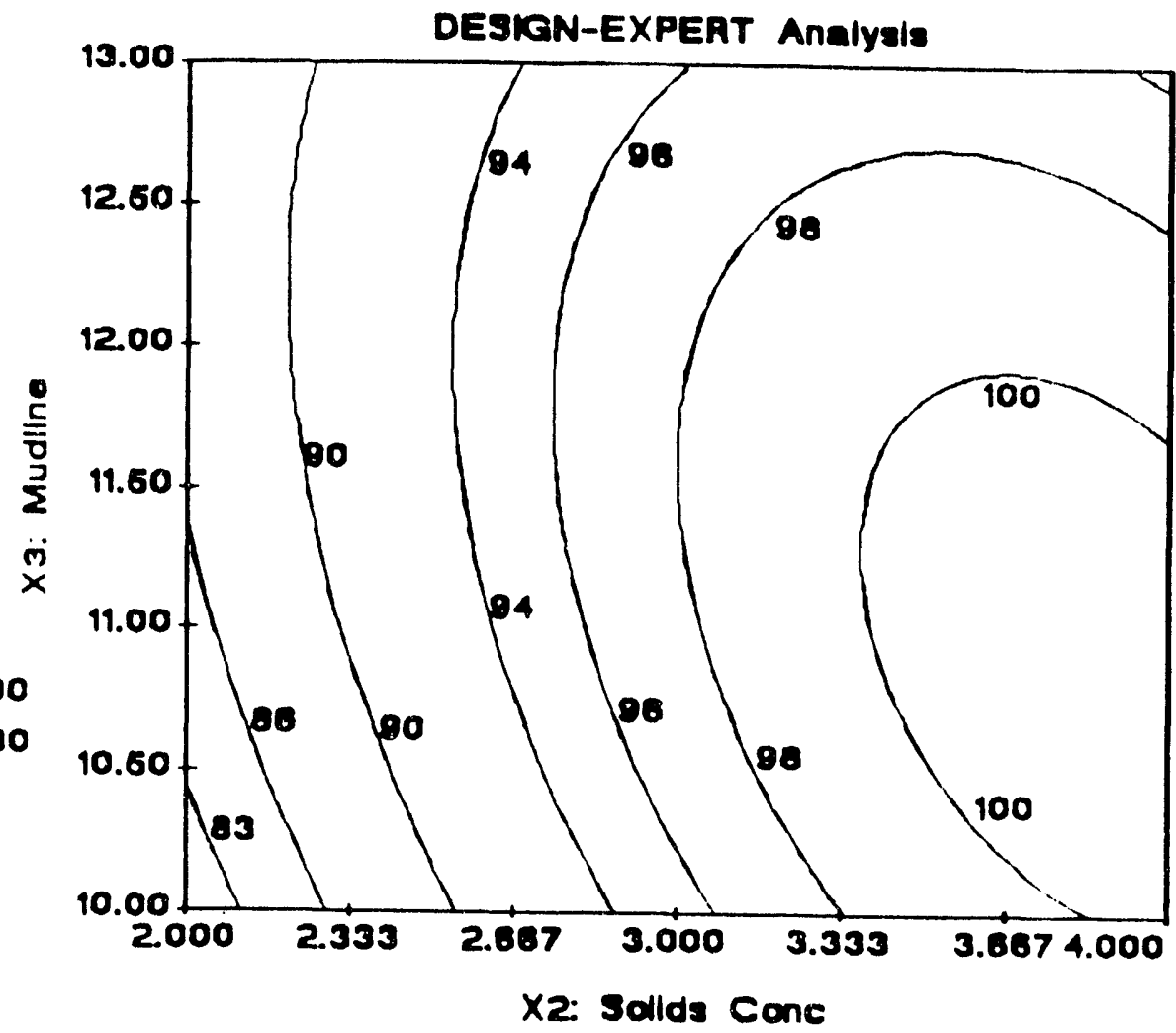

Figure B10: Combustible Recovery as a function of Feed Solids Content and Mudline Depth. 


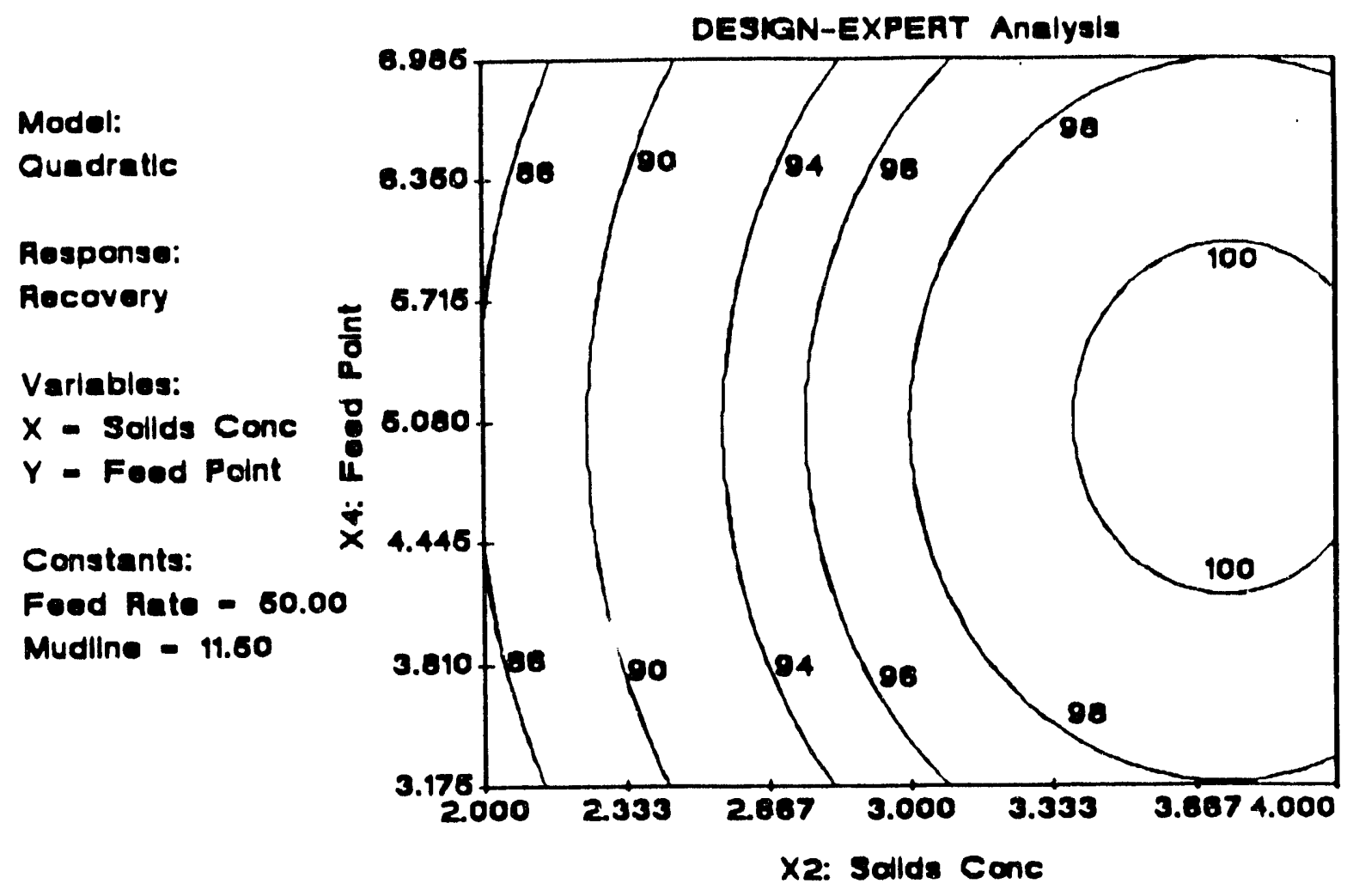

Figure B11: Combustible Recovery as a function of Feed Solids Concentration and Feed Inlet Point.

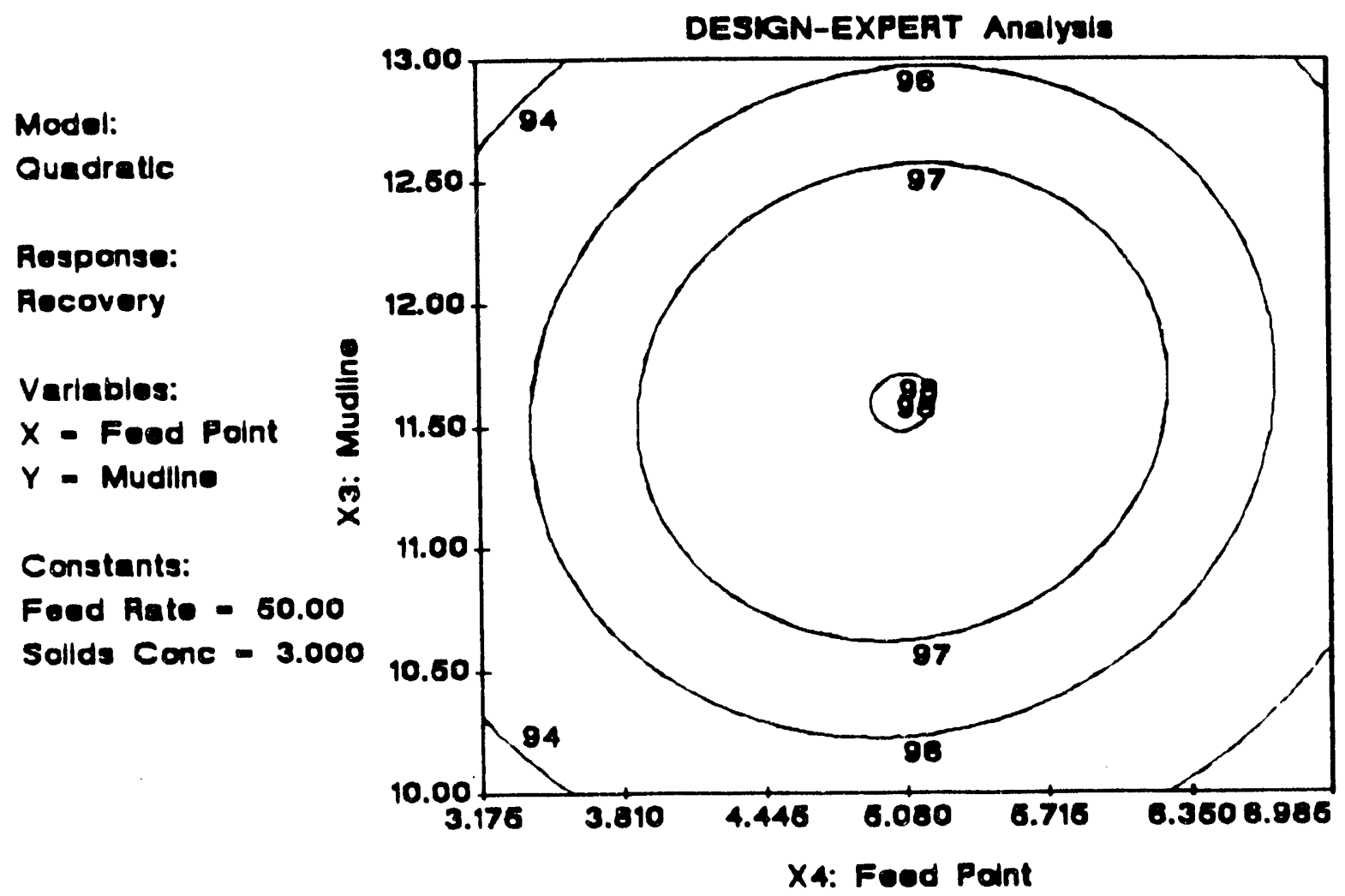

Figure B12: Combustible Recovery as a function of Mudline Depth and Feed Inlet Point. 


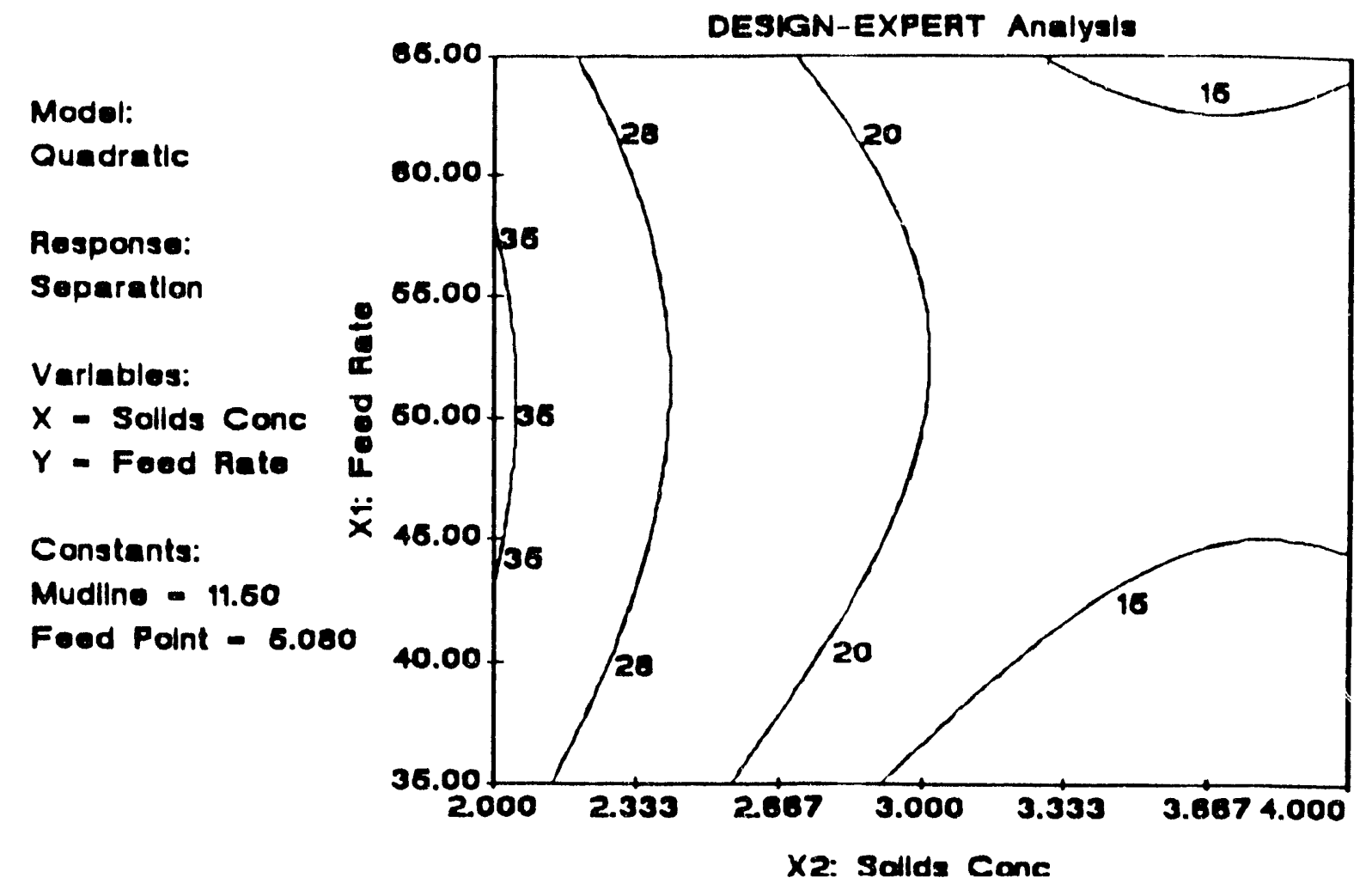

Figure B13: Separation Efficiency as a function of Feed Rate and Feed Solids Concentration.

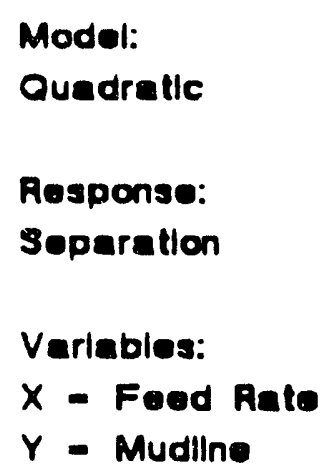

Constants:

Sollds Conc -3.000 Foed Point - 6.000

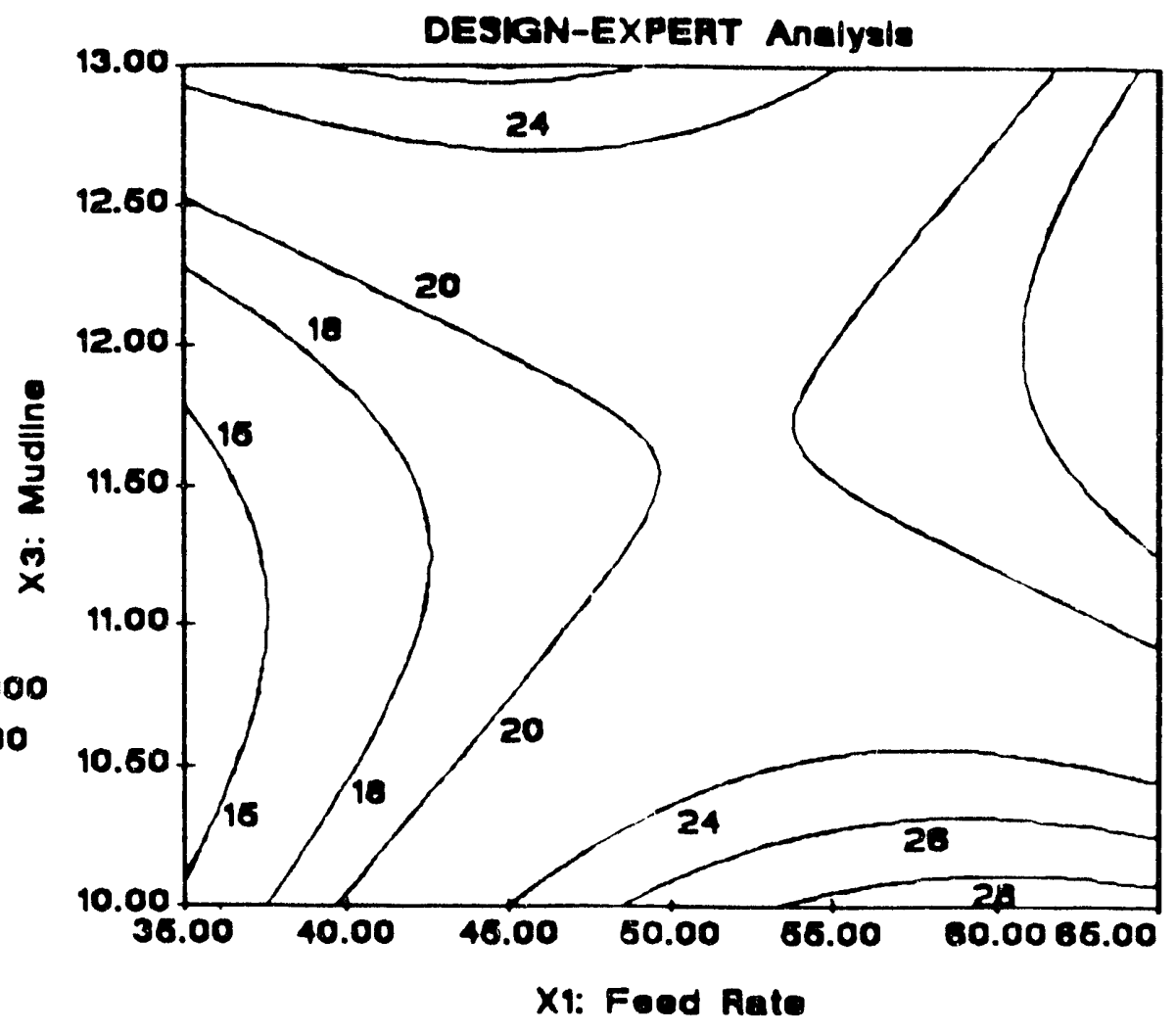

Figure B14: Separation Efficiency as a function of Feed Rate and Mudline Depth. 
Model:

Quadratic

Response:

Separation

Variables:

$X$ - Foed Rate

$Y$ - Foed Point

Constants:

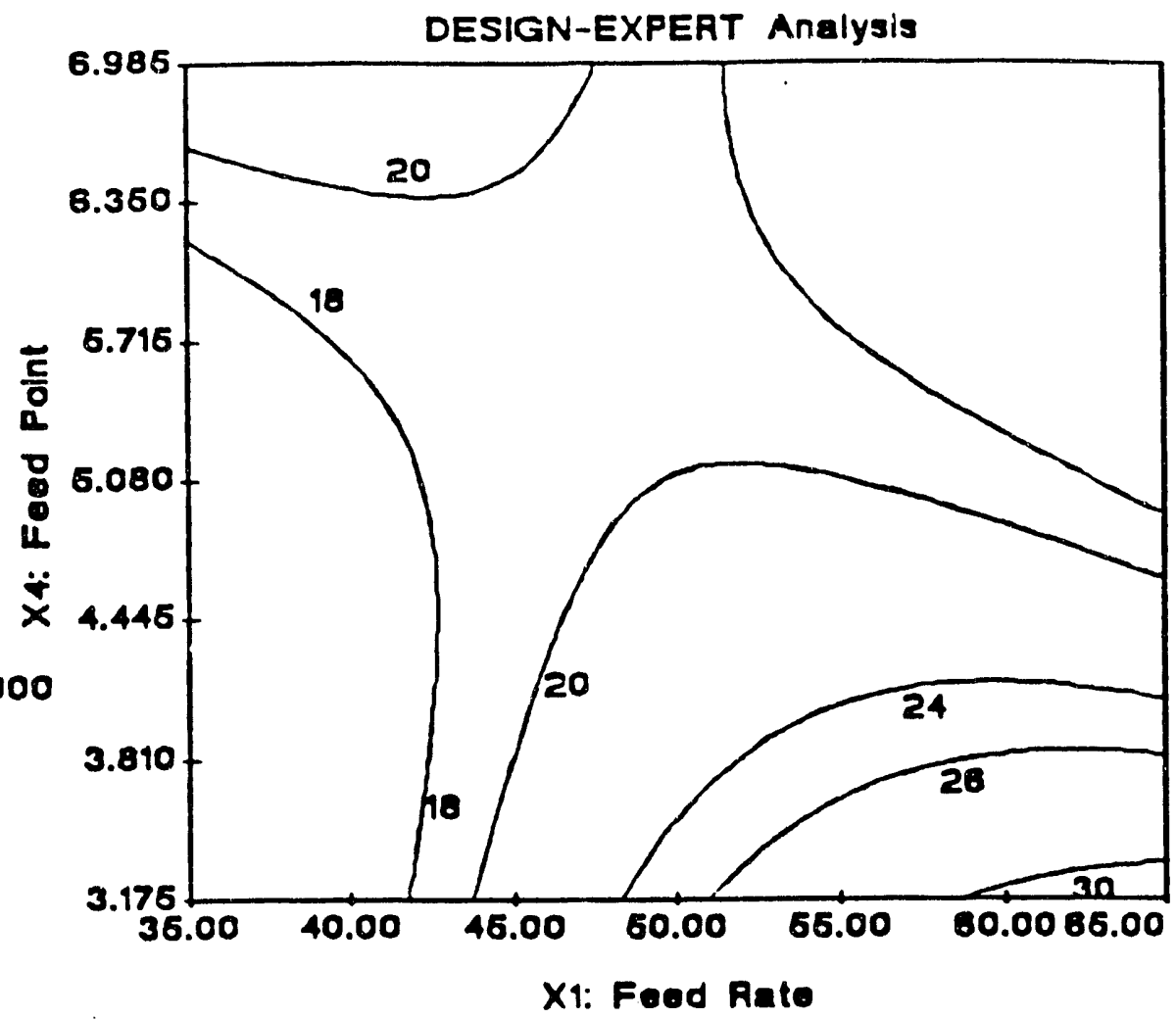

Figure B15: Separation Efficiency as a function of Feed Rate and Feed Inlet Point.
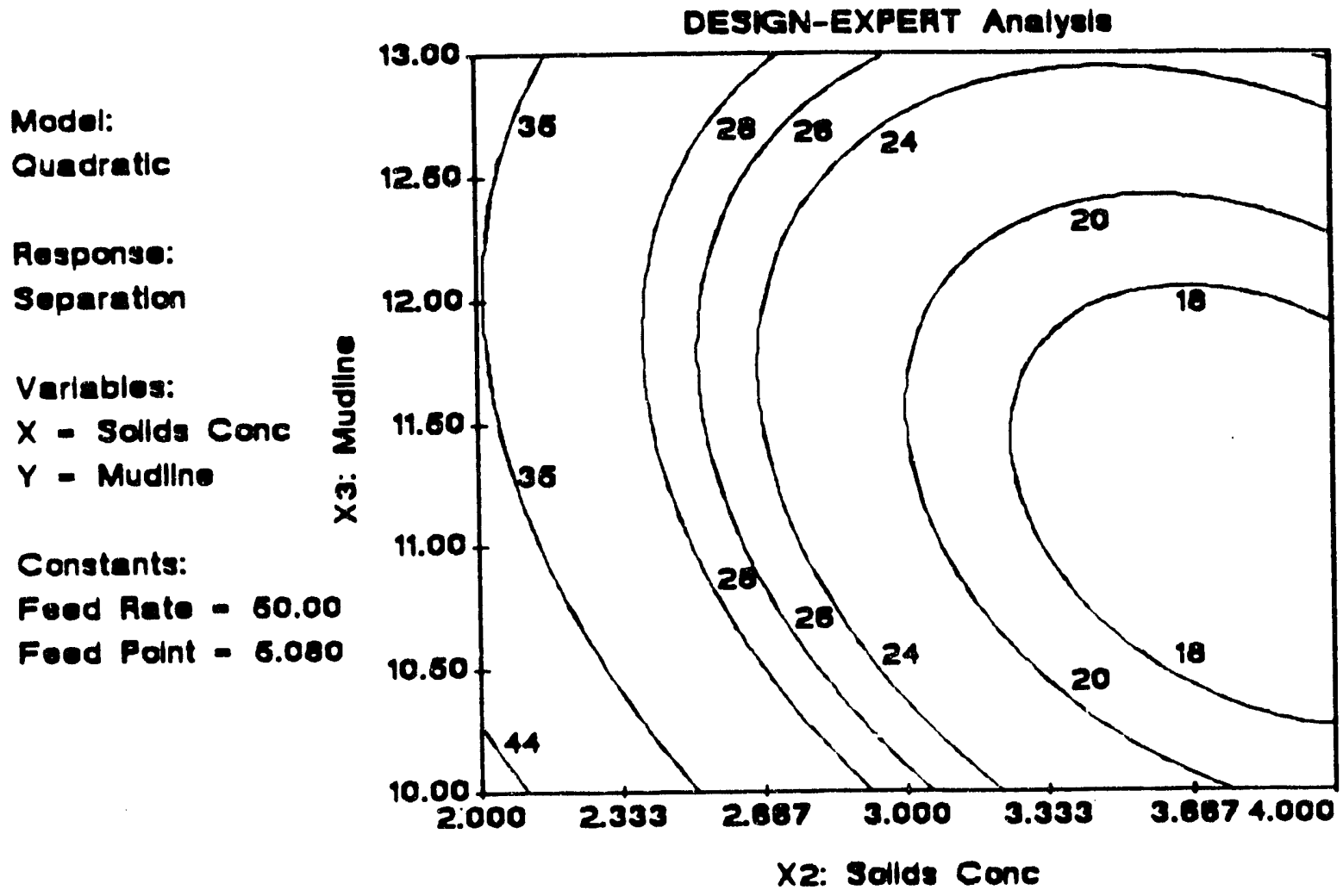

Figure B16: Separation Efficiency as a function of Feed Solids Concentration and Mudline Depth. 


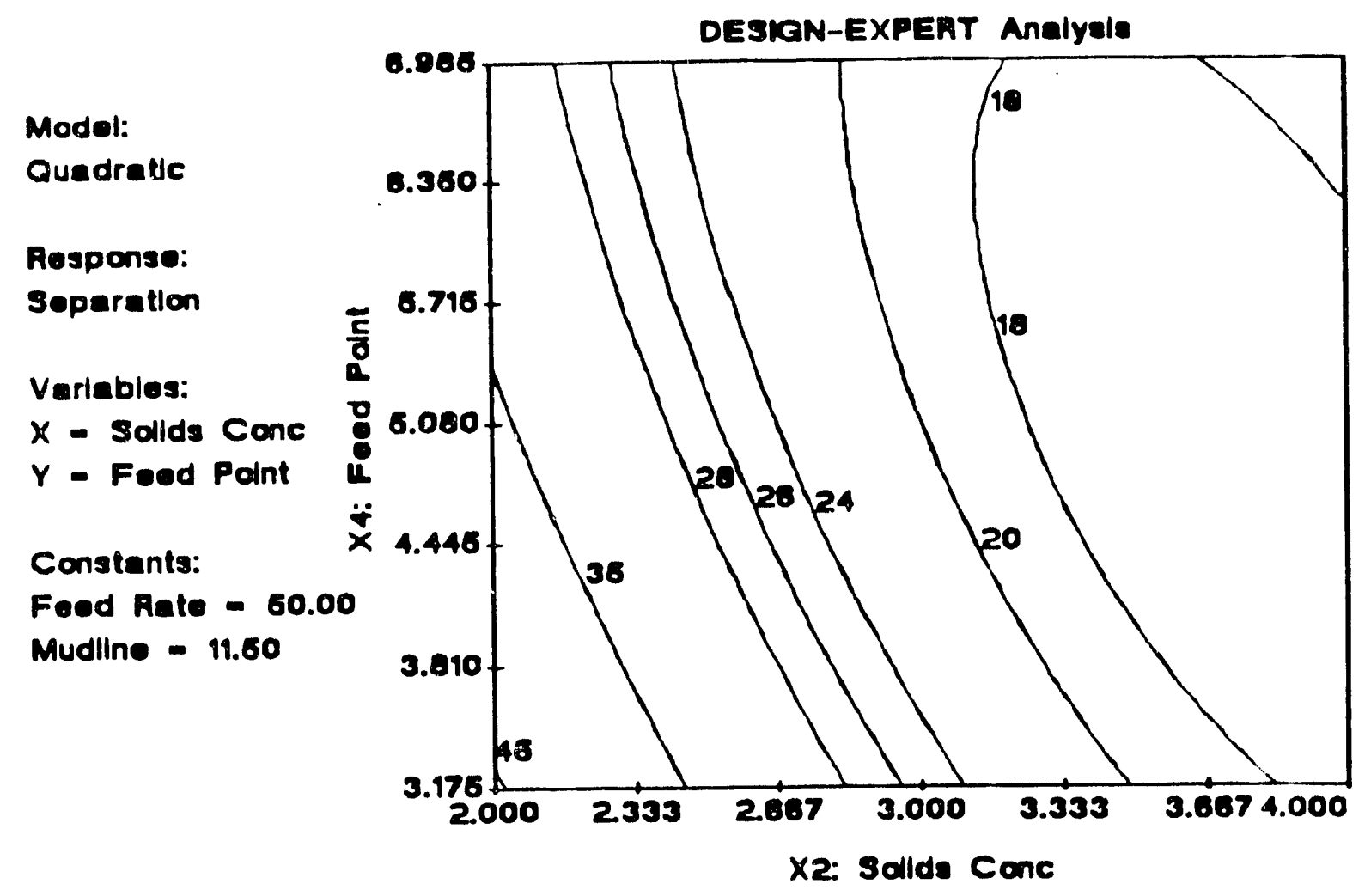

Figure B17: Separation Efficiency as a function of Feed Solids Concentration and Feed Inlet Point.

Model:

Quadratic

Response:

Soparation

Variables:

$X$ - Food Point

$Y$ - Mudilne

Constants:

Foed Rate - $\mathbf{6 0 . 0 0}$

Sollds Conc - 3.000

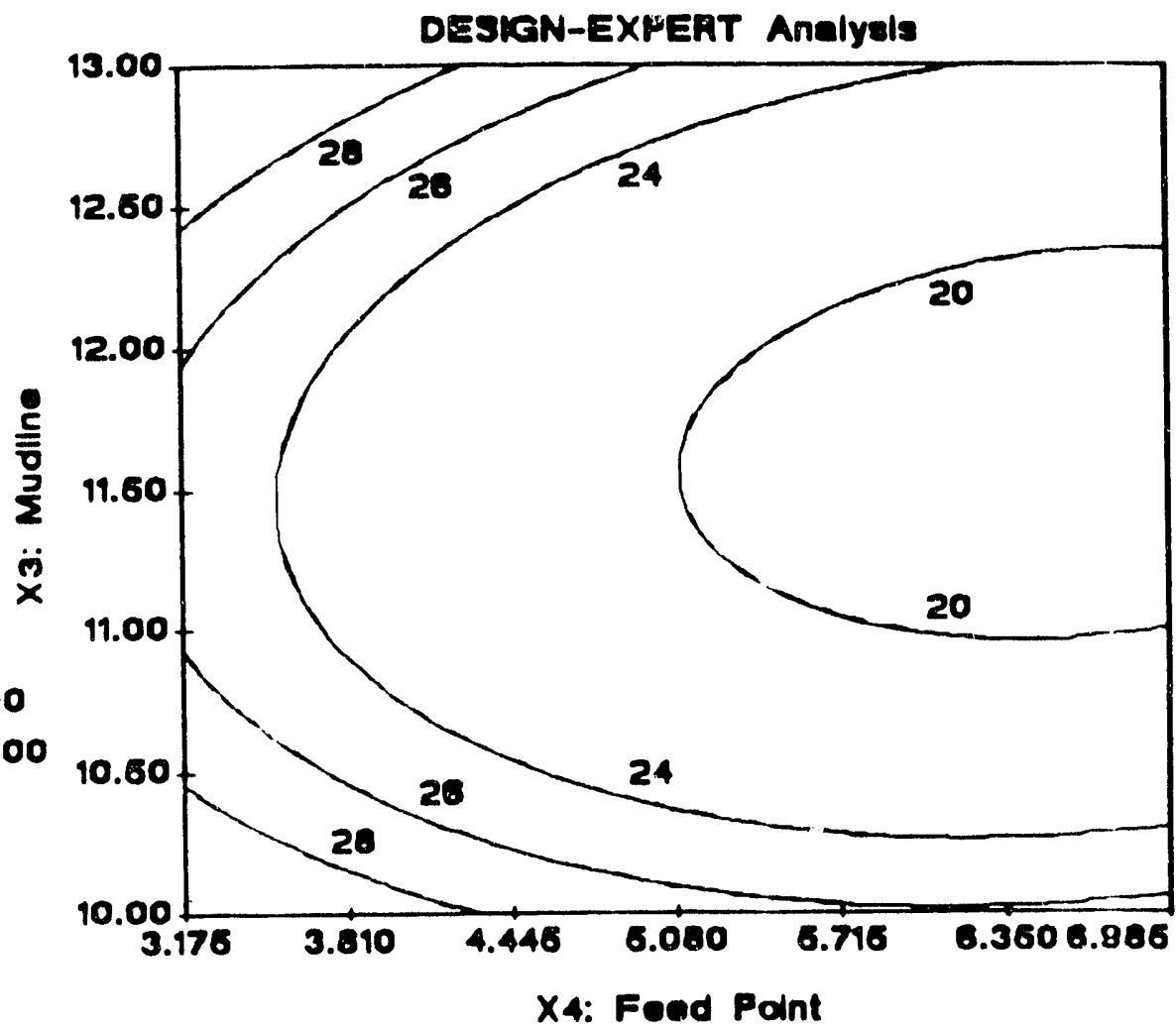

Figure B18: Separation Efficiency as a function of Mudline Depth and Feed Inlet Point. 

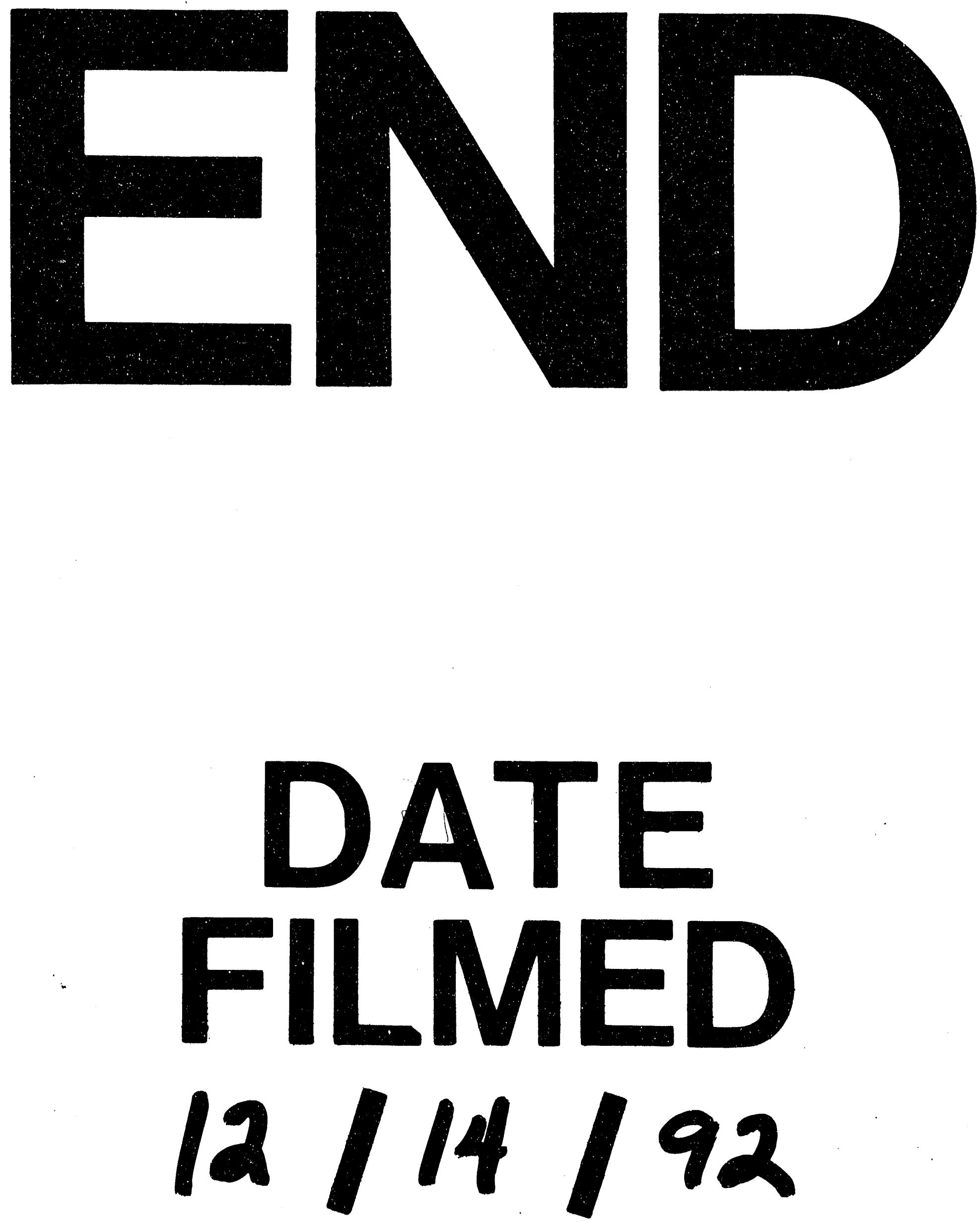
